Christian Kanzian

\title{
Alltagsontologie Eine metaontologische Grundlegung
}



Christian Kanzian

Alltagsontologie Eine metaontologische Grundlegung 
Christian Kanzian

Institut für Christliche Philosophie, Universität Innsbruck

Diese Publikation wurde mit finanzieller Unterstützung der Katholisch-Theologischen Fakultät und des Vizerektorats für Forschung der Universität Innsbruck gedruckt.

(C) innsbruck university press, 2020

Universität Innsbruck

1. Auflage

Alle Rechte vorbehalten.

Coverbild: Johannes Trager

www.uibk.ac.at/iup

ISBN 978-3-99106-021-5 


\section{Inhalt}

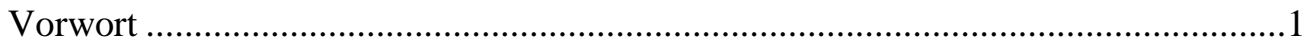

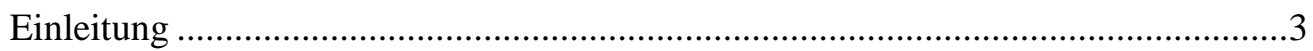

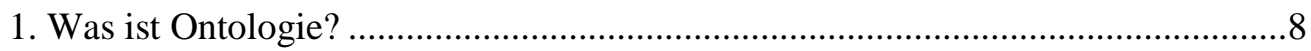

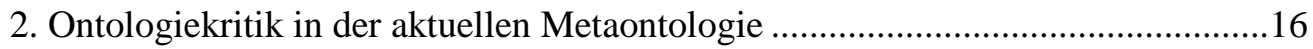

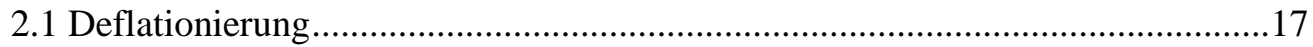

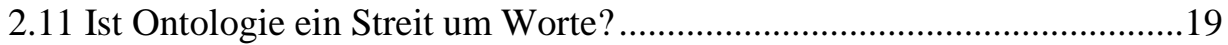

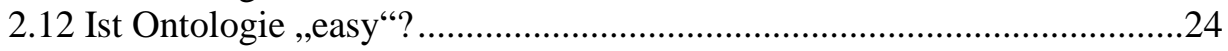

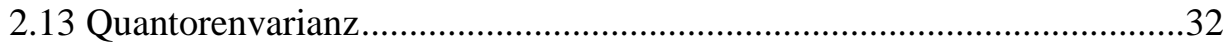

2.14 Die Nicht-Substantialität ontologischer Fragen....................................40

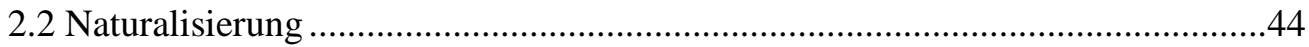

2.21 Die Metaphysikkritik der frühen Analytischen Philosophie.....................44

2.22 Die Sinnlosigkeit aller ,unbescheidenen Ontologie“ “.............................48

2.23 Die Nicht-Substantialität nicht-naturalistischer Ontologie .......................57

2.24 Die Unvollständigkeit nicht-naturalistischer Ontologie .........................61

2.3 Simplifizierung ........................................................................................66

2.31 Simplifizierung und das Ausblenden naturwissenschaftlicher

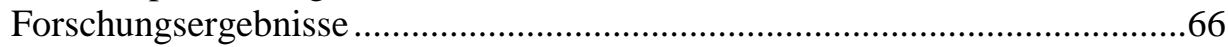

2.32 Simplifizierung unter dem Vorzeichen naiver Hypostasierungen

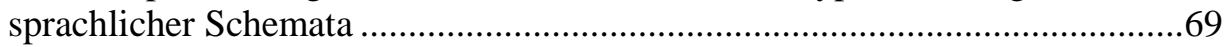

2.33 Simplifizierung als Einschränkung ontologischer Fragestellungen..........82

2.4 Ontologiekritik: ein Resümee....................................................................92

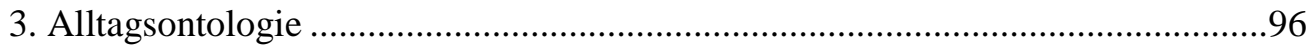

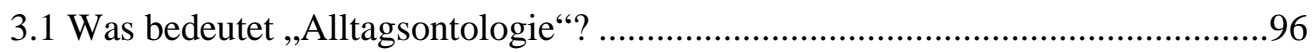

3.11 Peter Strawsons deskriptive Metaphysik ..............................................97

3.12 Grundkriterien für Alltagsontologie .....................................................102

3.13 Ontologie als Alltagsontologie ……..................................................109

3.2 Alltagsontologie und Ontologiekritik ........................................................115

3.21 Alltagsontologie und Deflationierung …...........................................115

3.22 Alltagsontologie und Naturalisierung ................................................136

3.23 Alltagsontologie und der Verdacht der Simplifizierung .........................164

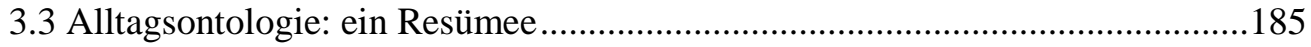

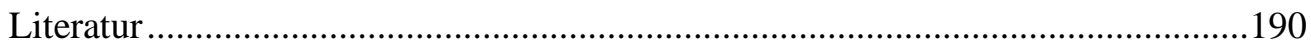

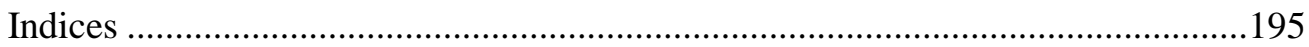





\section{Vorwort}

Der vorliegende Band ist als erster Teil einer Summe der Ontologie konzipiert. Ziel ist eine Gesamtdarstellung der Ontologie, welche zusammen mit dieser metaontologischen Grundlegung eine darauf aufbauende kategoriale Ontologie und eine angewandte Ontologie umfassen soll.

Dieses Buch ist dennoch ein abgeschlossenes Projekt. Es erhebt den Anspruch, Thesen zu vertreten, die ohne Bezug auf die Gesamtdarstellung kritisierbar sein sollen. Sein Anliegen ist die Entwicklung einer Alltagsontologie in kritisch-konstruktiver Auseinandersetzung mit Vertreterinnen und Vertretern der aktuellen metaontologischen Debatte. Mit dem Stichwort „Alltagsontologie“ ist auch die Leitidee der intendierten "Summe einer Ontologie“ auf den Punkt gebracht. Die Grundstrukturen der kategorialen Wirklichkeit finden wir weder an einer mikrophysikalischen Basis, noch in einem platonischen Ideenhimmel. Ontologie ist eine systematische Beschreibung der alltäglichen Lebenswelt. Das dabei vorausgesetzte Verstehen von Ontologie näher darzulegen, ist Aufgabe des vorliegenden Buches. Die weiteren geplanten Bände sollen dann die Grundstrukturen der alltäglichen Lebenswelt darstellen, zweiter Band, bzw., dritter Band, eine Anwendung ontologischer Grundlagen auf spezielle Lebensbereiche, aber auch auf Einzelwissenschaften und außerontologische philosophische Disziplinen versuchen.

Der Summen-Charakter der intendierten Gesamtdarstellung wird vor allem im zweiten Band verstärkt zur Geltung kommen, und zwar deshalb, weil der Autor dort auf bereits von ihm verfasste Bücher, gemeint sind die Monographien Ding - Substanz - Person und Wie Dinge sind, zurückgreifen kann. Mit dem vorliegenden ersten Band betrete ich Neuland. Hier werden die Darlegungen weitgehend, d.h. mit einigen gekennzeichneten Ausnahmen, ohne Referenzen auf selbst Verfasstes auskommen. Damit wird auch der Charakter dieser metaontologischen Grundlegung einer Alltagsontologie als geschlossener Monographie unterstrichen.

Grenzen hat natürlich auch die vorliegende Metaontologie. Eine, zu der sich der Autor ausdrücklich bekennen möchte, ist, dass Alltagsontologie ausschließlich im kritischen Diskurs, siehe oben, entwickelt wird. Mit Ausnahme 
der deskriptiven Metaphysik Strawsons werden somit keine alternativen alltagsontologie-nahen Positionen dargestellt, obwohl sich in Geschichte und Gegenwart sicherlich einige finden ließen. Diese Einschränkung hängt v.a. damit zusammen, dass das Augenmerk auf den Versuch systematischer Argumentation gelegt wird, nicht auf historische und vergleichende Analysen.

Die vorliegende metaontologische Grundlegung einer Alltagsontologie ist natürlich dennoch in einem Kontext entstanden. Dieser ist in erster Linie geprägt durch die starke Tradition in Metaphysik und Ontologie am Institut für Christliche Philosophie in Innsbruck. Wer zwischen den Zeilen zu lesen vermag, wird in diesem Buch Anleihen an Transzendentaler Metaphysik finden, insofern diese ausgehend von einer Untersuchung des praktischen Vollzugs bestimmter menschlicher Vermögen, des Urteilens oder des Fragens, die Grundthemen der Metaphysik und der Ontologie, Sein und Existenz, angeht. Viel offensichtlicher ist freilich die Orientierung an der aktuellen analytischen Ontologie, wie sie ebenfalls in den letzten Jahren und Jahrzehnten an unserem Institut gepflegt wurde. Damit kann ich eigentlich auch schon nahtlos zum Ausdruck geschuldeten Danks kommen: an die Altvorderen, namentlich seien Emerich Coreth, Otto Muck und Edmund Runggaldier erwähnt, aber auch an die Kolleginnen und Kollegen am Institut für viele ontologisch inspirierende Gespräche, last but not least an die Studierenden meiner Lehrveranstaltungen zur Ontologie v.a. in Innsbruck und in Brixen. Außerhalb Innsbrucks schulde ich vor allem meinem Kollegen Miroslaw Szatkowski Dank, der mit seinen hochkarätigen Konferenzen einen wesentlichen Beitrag zu jener Etablierung der analytischen Ontologie in Europa geleistet hat, der sich auch diese Arbeit verpflichtet weiß.

Besonderer Dank gebührt Herrn Matthias Thomas Troger für die sorgfältige Lektüre von Vorgängerversionen dieses Buches, für zahlreiche stilistische, aber auch inhaltliche Verbesserungen. Verbleibende Fehler liegen selbstverständlich in der alleinigen Verantwortung des Verfassers. Danke schließlich auch an innsbruck university press für die Aufnahme des Bandes in ihr open access-Programm und an die Theologische Fakultät für die finanzielle Unterstützung zur Deckung der Entstehungskosten.

Innsbruck, im Jahr 2020 


\section{Einleitung}

Peter van Inwagens richtungsweisender Artikel „Meta-Ontology“ ${ }^{\text {“1 }}$ hat aufs Neue die Diskussion darüber angeregt, was es denn überhaupt bedeute, sich ontologische Fragen zu stellen. Die Ontologie hat offensichtlich ein Reifestadium erreicht, so könnte man das wohlwollend interpretieren, zu dem es sich lohnt, innezuhalten und eine Reflexionsstufe höher, auf eine Metaebene zu steigen. Im Brennpunkt der von van Inwagen inspirierten Debatte steht die Frage nach der Existenz, und damit das Problem, was es bedeutet, von etwas zu sagen, es existiere. In der Analytischen Philosophie wird diese Frage naturgemäß auf die Verwendung von Quantoren in einschlägigen Kontexten fokussiert: Heißt „zu sein“ oder ,zu existieren“ schlicht und einfach im Wertebereich von Quantoren vorzukommen, wie wir von Quine lernen? ${ }^{2}$ Oder ist es nicht ein grobes Missverständnis, den fregeanischen Nicht-0-Quantor, ${ }^{3}$ wie man leicht ironisierend auch sagen könnte, für das tiefe Problem des Seins oder Nichtseins zu missbrauchen? ${ }^{4}$ Wenn der Wertebereich-Ansatz ungangbar ist, stellt sich die Frage, ob es eine Alternative gibt: Was mag es sonst bedeuten zu existieren? Ist hingegen der Quantoren-Weg grundsätzlich verfolgenswert: Wie ist das ,to be is to be the value of a bound variable“ genau genommen zu verstehen? Nehmen wir an, es gibt verschiedene Arten oder Kategorien von Existierendem. Gibt es dementsprechend auch verschiedene variablenbindende Quantoren? Nimmt man nur einen Typ Quantoren an, heißt das, dass man dementsprechend auch nur eine Art oder Kategorie Entitäten akzeptieren kann? Grundlegender: „Verpflichtet“ die Verwendung von Quantoren in jedem Fall darauf, die Elemente ihres Wertebereichs als Bestandteile der außersprachlichen oder außergedanklichen Wirklichkeit anzunehmen, oder gibt es auch so etwas wie ,leichtgewichtige“ (engl.: lightweight) Quantoren, die man in einem ontologisch ,unschuldigen“ Sinn verwenden darf?

\footnotetext{
${ }^{1}$ Hier: van Inwagen 1998.

${ }^{2}$ Hier geht es natürlich, wie gleich noch ausdrücklich gemacht wird, um Quine 1948.

${ }^{3}$ Klassisch: Frege 1884, $§ 52-55$.

${ }^{4}$ Vgl. dazu u.a. Tegtmeier 1997, 44. Dort spricht der Autor von „Mindestenseinsquantifikation“, um zu verstehen zu geben, dass mit der Fregeanischen Quantifikation das Seins- oder Existenz-Thema nicht erreicht werden könne.
} 
Im Alltag, aber auch in der Wissenschaft, reden wir ja oft, vermutlich sogar großteils, über etwas, das wir genau genommen gar nicht als real erachten, zumindest nicht in einem engen oder wörtlichen Sinn. Mit „Es gibt dicke Luft im Hause Maier“" meinen wir ebensowenig eine Entität wie mit der Aussage „Die Zahl $\pi$ ist irrational“ - wobei es sich zumindest bei Letzterem schon um einen die Debatte bereichernden Zweifelsfall handelt. Schließlich: Kann es überhaupt Aufgabe einer philosophischen Disziplin sein, über derlei Probleme nachzudenken? Sollte man das nicht lieber den Einzelwissenschaften und anderen Fachbereichen überlassen? Dort wüssten die ExpertInnen ohnehin besser, was sie in ihren Theorien oder spezifischen Annahmen als Existierendes oder als Entitäten annehmen wollen oder auch nicht.

Peter van Inwagen hat diese und vergleichbare Debatten in die Gegenwart hinein angestoßen. ${ }^{5}$ Damit wurden freilich ihre Inhalte nicht neu erfunden. Die Themen Existenz, Existenzfragen, Relevanz von Existenzfragen haben in der Philosophie eine gute Tradition, auch in der analytischen. Hier ist dies zunächst einer Festlegung in den Principia Mathematica zu verdanken, wo Russell/Whitehead im Hinblick auf die Grundlagendebatte in der Mathematik die Frage nach Existenz thematisieren und auch gleich lösen, indem sie Existenz schlichtweg mit Nicht-Angehören zur Nullklasse, d.i. für sie Widerspruchsfreiheit, gleichsetzen. ${ }^{6}$ Der Nicht-0-Quantor ist also, von seinem Ursprung her, ein „Mindestens-ein-nicht-der-Nullklasse-Angehör-Quantor“ - was wie bereits angedeutet auch unter analytischen PhilosophInnen immer wieder argwöhnisch aufgenommen wird.

Die Brisanz von Existenzfragen hat sich dann vor allem in der sogenannten Carnap-Quine-Debatte verdichtet. Diese wird uns in den kommenden Abschnitten noch ausführlich beschäftigen. In dieser Einleitung darf nur daran erinnert werden, dass Rudolf Carnap bezüglich Existenzfragen die Unterscheidung zwischen sogenannten ,externen“ und ,internen“ eingeführt hat. ${ }^{7}$ Externe Existenzfragen betreffen, so Carnap, ausschließlich die Wahl be-

${ }^{5}$ Für einen ersten Überblick darüber, was van Inwagen tatsächlich angestoßen hat, darf Berto/Plebani 2015 empfohlen werden.

${ }^{6}$ Siehe dazu u.a. Mennes Artikel „Existenz in der Logik“ in Weingartner 1966, 58ff. In dieser Edition werden übrigens nicht wenige Themen und Positionen der aktuellen Metaontologie vorweggenommen.

${ }^{7}$ Richtungsweisend ist Carnaps „Empirism, semantics, and ontology“; hier: Carnap 1950. 
stimmter begrifflicher Rahmenbedingungen. „Gibt es Zahlen?“ wäre eine solche. Externe Existenzfragen sind rein pragmatisch zu entscheiden. Wenn es, etwa in der Mathematik, sinnvoll ist, Zahlen anzunehmen, dann soll man dort eine entsprechende Rahmensprache mit den dazugehörenden Regeln einführen. Die Frage, ob es denn nun unabhängig von diesen begrifflichen Festsetzungen Zahlen wirklich gebe, ist nach Carnap weder wahr noch falsch zu beantworten, sondern schlichtweg sinnlos. Von den externen Existenzfragen sind jedenfalls die internen zu unterscheiden. „Gibt es Primzahlen, die größer sind als 100?" wäre ein Standardbeispiel einer solchen internen Frage. Diese ist einfach zu entscheiden, und zwar logisch, in Anwendung der mit der Zahlensprache eingeführten Regeln. $\mathrm{Zu}$ beachten ist, dass Carnap auch begriffliche Rahmen annimmt, in deren Bereich Existenzfragen nicht logisch, sondern empirisch zu entscheiden sind. „Gibt es Dinge?“ etwa ist die Frage nach der Akzeptanz einer Dingsprache, in deren Kontext Existenzfragen, z.B. „Gibt es ein Stück Papier auf meinem Schreibtisch“, nicht logisch, sondern einfach empirisch zu beantworten sind. Jedenfalls verliert das Existenzthema das Flair des Metaphysischen: Existenzfragen sind entweder pragmatisch, und damit in einer kognitiv irrelevanten Weise zu entscheiden, oder aber einfach, und zwar logisch bzw. empirisch. Carnap wird damit zum Vorläufer des heute wieder hochangesehenen Programms der Deflationierung der Ontologie: Von der hochdekorierten Wissenschaft von der Existenz bleiben Pragmatik, Logik und empirischer Verifikationismus.

Willard Van Orman Quine geht es wahrlich nicht darum, die Ontologie zurück in den Stand einer Seinsmetaphysik zu versetzen. Dazu ist er seinem „Lehrer und Freund“ Carnap viel zu nahe. Dennoch ist Quine skeptisch gegenüber der carnapschen Weise der Deflationierung der Ontologie. Quine kritisiert problematische Prämissen in Carnaps Theorienbildung: die Unterscheidung zwischen analytischen oder logischen und synthetischen oder empirischen Urteilen und die damit zusammenhängende Annahme, dass man überhaupt einzelne Sätze verifizieren könne. ${ }^{8}$ Es kann hier nicht die Aufgabe sein, die (ohnehin sattsam bekannten) Detailargumente Quines darzulegen, schon gar nicht, diese kritisch zu bewerten, etwa darauf hin, ob und inwiefern Carnap durch die quineschen Einwände tatsächlich getroffen wird oder auch

\footnotetext{
${ }^{8}$ Siehe v.a. Quine 1951.
} 
nicht. Worum es hier geht, ist, den quineschen Hauptimpuls für die aktuelle metaontologische Debatte $\mathrm{zu}$ fokussieren. Dieser setzt bei seinem holistischen Theorienverständnis an: Verifiziert werden nach Quine niemals einzelne Sätze, sondern stets Theorien als Ganze. Da Theorien als Ganze nicht nur empirienahe Theoreme beinhalten, sondern immer auch gewisse logische oder andere formal-strukturelle Elemente, kann man gerade im Hinblick auf die Bestätigung von Theorien die Trennlinie zwischen den empirisch-synthetischen und den logisch-analytischen Theorieteilen nie exakt ziehen. In diesem Sinne sind sogar logische Regeln Gegenstand der Verifikation, wohingegen auch klar Sinneseindrücken zuordenbare Aussagen durch scheinbar gegenläufige empirische Befunde nicht von vornherein zu falsifizieren sind. Es geht also um Theorien als ganze. Was aber sind jene ganzen Theorien, die Quine in besonderem Maße interessieren und für ihn den Inbegriff von Wissenschaftlichkeit ausmachen? Es sind die Theorien der Naturwissenschaften, allen voran der Physik auf ihrem aktuellen Forschungsstand. Dementsprechend sieht Quine die Aufgabe der Ontologie auch darin, jene ontologischen Verpflichtungen in den Blick zu bekommen, die man mit den besten wissenschaftlichen Theorien eingeht. Man muss annehmen, dass es das gibt, worüber man in diesen Theorien spricht. Worüber man spricht, wird bei Quine freilich nicht durch jene singulären Termini festgelegt, die wir im Alltag verwenden, etwa Eigennamen wie „Pegasus“ oder Kennzeichnungen wie „der gegenwärtige König von Frankreich“. Es sind vielmehr jene Variablen (,,etwas"), von denen schon die Rede war: nämlich die durch den sogenannten Existenzquantor (,es gibt“") gebundenen. Dass es etwas gibt oder dass etwas existiert, bedeutet somit nach Quine nichts anderes, als dass es im Referenzoder Wertebereich gebundener Variablen vorkommt. ${ }^{9}$ Das aber sind, und damit kommen wir zu Quines faktischem Physikalismus, jene Entitäten, auf welche die besten wissenschaftlichen Theorien Bezug nehmen: physikalische Objekte (engl.: physical objects).

Quines physikalistisch inspirierte Quantoreninterpretation und Carnaps Deflationierungsprogramm machen die Eckpfeiler der aktuellen metaontologischen Debatte aus. Es ist nicht übertrieben $\mathrm{zu}$ behaupten, dass ohne die

${ }^{9}$ Siehe Fußnote 2. 
grundlegenden Impulse dieser beiden Autoren die zeitgenössischen Diskussionsbeiträge, so kreativ und innovativ sie im Detail auch sein mögen, nicht $\mathrm{zu}$ verstehen sind.

Dennoch soll es hier keinesfalls um Carnap- bzw. Quine-Exegese gehen, sondern vielmehr darum, einen speziellen Begriff der Ontologie, nämlich einer deskriptiven Ontologie bzw. einer Alltagsontologie zu entwickeln, und zwar in Auseinandersetzung mit den aktuellen metaontologischen Debatten. Das soll so geschehen, dass wir mit der Darlegung eines Einstiegsbegriffs von Ontologie beginnen (Kapitel 1.). Dann werden Bedenken gegen die Ontologie aus der von Carnap und Quine geprägten Metaontologie dargestellt (Kapitel 2.). In Auseinandersetzung mit diesen Einwänden soll, ausgehend vom Einstiegsbegriff, das spezielle oder besondere Verstehen von Ontologie als Alltagsontologie herausgearbeitet werden (Kapitel 3.). Damit sollen jene Rahmenbedingungen geklärt werden, in deren Kontext eine bestimmte kategoriale Ontologie, in der weiteren Folge auch Anwendungsbedingungen einer solchen im Sinne einer angewandten Ontologie entwickelt werden können. Das aber weist schon weit über das nunmehr zu Behandelnde hinaus. 


\section{Was ist Ontologie?}

Es hätte natürlich seine Berechtigung, die in der Überschrift gestellte Frage zunächst unter historischer Perspektive ins Auge zu fassen. Wie haben führende Exponenten der Ontologie die Grundanliegen ihrer Disziplin verstanden? Vermutlich müsste man dabei mit Parmenides beginnen, seiner lapidarprogrammatischen Feststellung, dass das Sein ist, das Nichtsein eben nicht, und den sich daraus ergebenden Paradoxien, u.a. bezüglich des Werdens und Vergehens. Daraus ließe sich der theoretische Rahmen für das platonische, in der Folge auch das aristotelische Philosophieren darlegen und, beim Stagiriten, gleich fokussieren auf ein weiteres bahnbrechend die Ontologie charakterisierendes Diktum, dass es nämlich eine Wissenschaft gebe, die das Seiende als Seiendes analysiere. Wenn man dann die mittelalterliche Scholastik in ihrem faszinierenden Ideenreichtum bei der Entfaltung der griechischen Seinslehre ausblendete, könnte man gleich übergehen zum neuzeitlichen Begriff einer Ontologie, insofern er bei Christian Wolff wohl zum ersten Mal als terminus technicus für eine Disziplin der metaphysica generalis eingeführt wurde. Besonders lohnen würde es sich, mit dem Vorurteil aufzuräumen, dass mit Wolffs Beginn auch gleichzeitig das Ende der philosophischen Ontologie angebrochen wäre, angesichts der vermeintlichen Desavouierung durch Kant sowie seine Nachfolger im Deutschen Idealismus und in den verschiedenen neukantianischen Strömungen. All dies möchte ich nicht tun. Und zwar deshalb nicht, weil es hier definitiv nicht um Philosophiegeschichte geht.

Das heißt natürlich nicht, dass Versuche, systematisch die Ontologie zu entfalten, vollkommen losgelöst von bestimmten historischen Kontexten geschehen könnten. Bei philosophischem Arbeiten ist diese Möglichkeit ja grundsätzlich fraglich, im Fall des Vorliegenden auch gar nicht erwünscht. Der hier vorzutragende Ansatz weiß sich insbesondere der aristotelischen, aber auch der kantischen Philosophie und ihren vielfältigen Traditionen verpflichtet. Dass es trotzdem nicht um Philosophiegeschichte geht, meint die grundsätzlich systematische Ausrichtung der vorgebrachten Überlegungen, die ohne exegetischen Anspruch vorgetragen werden. 
In diesem Sinne möchte ich den Einstieg für die Darlegung eines allgemeinen Begriffs der Ontologie auch nicht bei einer historisch belegbaren Definition, sondern bei einem sehr praxisnahen Faktum versuchen. Dieses besteht in der Feststellung, dass es im Grunde genommen keine menschliche Tätigkeit geben kann, die nicht irgendwelche Voraussetzungen bzgl. dessen machen würde, was es gibt. Dieses Faktum soll zunächst so unspezifisch vorgetragen werden, dass damit unterschiedslos sowohl die alltägliche als auch die wissenschaftliche, einschließlich der philosophischen Praxis beschrieben werden kann.

Bezüglich des Ersten, der alltäglichen Praxis, bedarf es lediglich einer gewissen reflexiven Aufmerksamkeit und der Bereitschaft, sich nach einer Schrecksekunde ein Stück weit auf das Unvermeidbare, sprich einen Wust von Existenzannahmen, einzulassen. Der Wecker läutet, ich schaffe es aufzustehen, kontrolliere die meteorologischen Gegebenheiten der Außenwelt und entschließe mich, vielleicht gegen innere Widerstände, trotzdem einen Arbeitstag in Angriff zu nehmen. Lassen wir die Fundamentalfrage beiseite, ob es nicht besser wäre im Bette zu bleiben, wenn wir annehmen würden, dass es ohnehin nichts gibt und nicht vielmehr etwas; so bleiben immer noch Kaffeemaschine, Tageszeitung, strahlender Sonnenschein, eine mögliche Busfahrt, ja die deutlich in mir aufkommenden Unlustgefühle, mich in ein Büro zu begeben, welche praxisleitende Vorannahmen bzgl. deren Existenz oder auch Nichtexistenz verlangen, auch wenn deren Explizitmachung frühmorgens faktisch unmöglich ist.

Die wissenschaftliche Praxis ist natürlich ungleich diffiziler und für PhilosophInnen vermutlich noch wesentlich schwerer zu durchschauen, auch bei fortschreitendem Tag. Dennoch dürfen wir aus verlässlicher Quelle annehmen, dass es wohl keine Mathematik gäbe, ohne irgendwelche mit „,ist“ oder „gibt" versehenen Annahmen bzgl. Zahlen, Mengen oder Klassen; keine Physik ohne solche Einstellungen über gewisse Partikel, Felder, Quanten; keine Sozialwissenschaften ohne Institutionen und deren Gesetzmäßigkeiten; ja nicht einmal eine Meteorologie ohne die Annahme von Hoch- und Tiefdruckgebieten. Auch EinzelwissenschaftlerInnen müssen mit etwas umgehen, und das, was sie annehmen, womit sie umgehen, ist ganz maßgeblich für ihre theoretische Praxis und deren Ergebnisse. 
Auch philosophische Tätigkeit jeder Art ist von derlei praxisbestimmenden Annahmen bzgl. dessen, was es gibt oder nicht gibt, keinesfalls ausgenommen. Selbst die/der hartnäckigste KonstruktivistIn beispielsweise muss annehmen, dass sie/er konstruiert, und er/sie könnte wohl nicht konstruieren, ohne dass es irgendetwas, zumindest ihn/sie selbst gibt, das die Tätigkeit der Konstruktion vollzieht. Und nicht nur das: Er/Sie muss auch annehmen, dass es das, was er/sie konstruiert, unabhängig davon, dass er/sie es konstruiert, gar nicht gibt. Und schon sind wir inmitten von manifesten, sogar äußerst schwerwiegenden positiven, aber auch negativen Seins- oder Existenzannahmen. Analoges könnten wir über die BetreiberInnen von Erkenntnistheorie sagen, insbesondere einer solchen, die Ontologie als hinter das kantische Reflexionsniveau zurückfallend brandmarken würde.

Das alles klingt sehr einfach. Bis zu einem bestimmten Punkt ist es das auch. Dass wir keine Tätigkeiten ausführen können, ohne bestimmte Voraussetzung bzgl. dessen, was es gibt, zu machen, ist eine einfache These. Sie ist einfach, im Sinne von grundlegend und keiner vorhergehenden Analyse zugänglich. Naiv ist sie aber, und das möchte ich von vornherein klarstellen, dennoch nicht. Mit dem Hinweis auf die besagte einfache These wird nämlich sicher nicht vorentschieden, was denn das ist, das Seiende oder das Existierende. Es wird ja keinesfalls behauptet, dass es klar sei, was denn die Existenzannahmen bei den verschiedenen Praktiken sind, bzw. wie sich die Existenzannahmen dieser Praktiken, etwa der naturwissenschaftlichen und der altäglichen, zueinander verhalten. Ganz im Gegenteil: Hier beginnen eigentlich erst die ontologischen Dispute. So lässt sich trefflich darüber streiten, ob die mathematische Praxis tatsächlich auf die Existenz von Zahlen angewiesen ist. Nicht nur das: Auch das Sein von Kaffeemaschinen, von Tageszeitungen, natürlich auch von Hochdruckgebieten und schon gar von Unlustgefühlen wurde und wird mit Gründen in Frage gestellt. Ebenso ist darüber zu diskutieren, ob die Existenzannahmen der Physik grundlegender seien als jene der alltäglichen Praxis, oder nicht doch umgekehrt.

Hier könnte man nun den Ausgangspunkt der Ontologie als philosophischer Disziplin ansetzen: Sie ist die systematische Reflexion auf Voraussetzungen menschlicher Praxis bzgl. dessen, was ist oder existiert, mit dem Ziel, 
eine konsistente Rahmentheorie über die Klärung dieser Voraussetzungen zu erlangen. ${ }^{10}$

An dieser Stelle angelangt, können wir in der Folge dazu übergehen, den Begriff einer systematischen Reflexion im Kontext einer philosophischen Lehre von Sein bzw. Existenz zu entfalten.

Diese Entfaltung kann zunächst unter formaler Rücksicht geschehen. ${ }^{11} \mathrm{Da}$ ist in erster Linie zu erwähnen, dass man sich mit dem Betreiben von Ontologie auf die Übernahme einer fachspezifischen Terminologie verpflichtet. Im Kontext einer Abhandlung, der es um die Entwicklung einer Ontologie in Auseinandersetzung mit der eingangs geschilderten metaontologischen Debatte geht, legt sich jene Fachterminologie nahe, wie sie in der analytischen Ontologie eingeführt wurde. ${ }^{12}$ Carnap und Quine sind hier klarerweise wesentliche Bezugspunkte. Damit wird nicht behauptet, dass es nicht auch brauchbare nicht-analytische Begriffsbildungen für die Ontologie gäbe; ebensowenig, dass in der analytischen Ontologie ein Fachbegriffskatalog vorläge, der vollständig und allgemein akzeptiert wäre; lediglich, dass eine Reihe zentraler ontologischer Termini von den beiden erwähnten, aber auch anderen maßgeblichen Autoren (Strawson, Lowe, um nur zwei weitere zu nennen) so eingeführt sind, dass sich einheitliche Verwendungsweisen rekonstruieren lassen. Schließlich soll darauf aufmerksam gemacht werden, dass das Attribut ,analytisch“ in dem hier verwendeten Kontext in einem möglichst offenen und undogmatischen Sinn zu verstehen ist. So sei beispielsweise darauf verwiesen, dass in der angestrebten Entwicklung einer Alltagsontologie im drit-

\footnotetext{
${ }^{10}$ Ich sehe an dieser Stelle übrigens einen Zusammenhang mit jener Transzendentalen Metaphysik oder Ontologie, welche gerade durch die Reflexion auf die Bedingung der Möglichkeit bestimmter menschlicher Vollzüge, sei es jetzt des Urteilens (Maréchal), sei es des Fragens (Coreth), zum Aufweis eines - wie sie sagen - nicht relativierbaren Seinshorizonts kommen. Auch von diesem Ansatz her ergibt sich, dass es keine bzgl. Existenz voraussetzungslose Praxis gibt. Auch in der Transzendentalen Metaphysik wird übrigens aus diesem grundsätzlichen Einstieg die Frage nicht vorentschieden, welche Arten oder Kategorien von Seienden oder Entitäten tatsächlich anzunehmen sind. Das ist Sache weiterführender Analysen und theorienbildender Überlegungen. Zum Einstieg in Transzendentale Metaphysik sei Coreth 1999 empfohlen.

11 An dieser Stelle greife ich Überlegungen aus der Einleitung zu meinem Buch „,Ding Substanz - Person“ (hier: Kanzian 2009) auf.

${ }^{12}$ Grundlegend: Newen/Savigny 1996; Runggaldier/Kanzian 1998.
} 
ten Kapitel auch manche Elemente des späten Husserl und von ihm inspirierter Philosophen aufgegriffen werden.

„Philosophisch“ meint weiterhin, dass versucht wird, Gedanken und Theorien argumentativ zu entwickeln. Argumentativ heißt begrifflich klar und nachvollziehbar, nicht jedoch ausschließlich formal oder gar formalisiert. Auch Carnap und Quine, Strawson und Lowe sprechen durchaus in philosophischer Prosa. Das entspricht der Einstellung, dass Formalisierungen, etwa in Sprachen logischer Kalküle, äußerst nützliche Hilfsmittel sind, und, richtig angewandt, hinreichend sein mögen, um begrifflich klar und nachvollziehbar zu sein. Dennoch sind Formalisierungen nicht notwendig, um das Ziel der Klarheit und Nachvollziehbarkeit zu erreichen. Auch in diesem Sinne ist die anvisierte Vorgangsweise als offen zu charakterisieren. Natürlich auch deshalb, weil durchaus zugestanden werden darf, dass es auch außerhalb der landläufig als „,analytisch“ charakterisierten Literatur sehr klare und argumentativ nachvollziehbare ontologische Theorien gibt.

Jedenfalls braucht die systematische Reflexion auf die Existenzvoraussetzungen unserer Praxis eine klare begrifflich argumentative Grundlage. Ein Orientierungsrahmen ist die standardmäßige analytische Ontologie in Prosa.

Aus der versuchten Einstiegsdefinition von Ontologie lassen sich aber auch inhaltliche Eigenarten dieser Disziplin herleiten. Eine erste: Hat die Ontologie zum Ziel, eine Rahmentheorie der Voraussetzungen bzgl. dessen, was ist oder existiert, wie sie auf den verschiedenen Ebenen unserer Praxis gemacht werden, zu entwickeln, kann sie sich in ihrer systematischen Reflexion keiner Praxis verschließen. Es geht der Ontologie somit buchstäblich um alles, um gleich nochmals Quine zu bemühen. ${ }^{13}$ Das bedeutet, dass man in der Ontologie zunächst alles ernst nehmen muss, was dafür in Frage kommt, in einer Praxis als existierend vorausgesetzt zu werden. Was das ist bzw. worin dieses „alles“ besteht, ist freilich keinesfalls klar. So besteht eine der vornehmsten Aufgaben der Ontologie darin, vermeintlich in einer Praxis Vorausgesetztes von in dieser Praxis tatsächlich Vorausgesetztem zu unterscheiden. Streitigkeiten, was dem Ersteren, was dem Letzteren zuzuordnen ist, und wie eine solche Zuordnung begründet werden kann, erhalten die Ontologie am Leben.

${ }^{13}$ Gemeint ist natürlich der berühmte Beginn von Quine 1948. 
Die Ontologie ist also, wie manche auch sagen, Universalwissenschaft. Davon unberührt bleibt allerdings das Anliegen „regionaler Ontologien“, etwa einer Sozialontologie, einer Ontologie der Biologie, einer Ontologie virtueller Welten, um nur drei Beispiele anzuführen. Das Anliegen solcher regionalen Ontologien könnte man so interpretieren, dass es jeweils um die Existenzvoraussetzungen einer speziellen Praxis bzw. eines speziellen Zusammenhangs von Praktiken geht, schließlich auch um bestimmte gemeinsame Grundzüge verschiedener Praktiken - im Rahmen freilich einer Universaltheorie der Existenzvoraussetzungen menschlicher Praxis. Die gegebenen Beispiele sollten dies verdeutlichen. Einer Ontologie der Biologie geht es um die systematische Reflexion auf Existenzvoraussetzungen einer Einzelwissenschaft. In der Ontologie virtueller Welten ${ }^{14}$ spielen eine ganze Bandbreite von Praktiken eine Rolle, angefangen von der Computertechnologie, über die Praxis bestimmter interaktiven Spiele, bis hin zu neuen Gestalten zwischenmenschlicher Begegnungen. Diese bilden freilich einen inneren Zusammenhang, insbesondere im Hinblick auf die ontologische Frage nach der Existenz virtueller Welten und ihrer Bewohner. Als gemeinsamer Grundzug verschiedener Praktiken kann schließlich deren sozialer Aspekt angesehen werden. Wie ist dieser ontologisch zu verstehen? Existiert das Gesellschaftliche in der Vielfalt seiner Erscheinungsweisen? Wenn ja, was sind dessen Eigenarten? Antworten auf derartige Fragen werden in der Sozialontologie gesucht.

Kurzum: Regionale Ontologien haben ihre Berechtigung und stehen dem Anliegen einer Ontologie als Universalwissenschaft nicht entgegen.

Wir kommen damit zu einem anderen Charakteristikum ontologischer Reflexion. Diesem können wir uns durch die Frage annähern, was denn die Eigenart der Ontologie als universaler Rahmentheorie bzgl. Existenzvoraussetzungen ausmacht? Es könnte ja auch andere Projekte geben, die mit dem Anspruch der Universalität Existenzfragen angehen, etwa einzelwissenschaftliche. Eine klassische, aber dennoch auch zeitgemäße Antwort ist, dass es der Ontologie um alles geht, insofern es ist oder existiert. Die Ontologie liefert eine allgemeine Rahmentheorie bzgl. Existierendem. Das lässt sich dahingehend erläutern, dass diese Theorie ohne Einschränkungen durch bestimmte

\footnotetext{
${ }^{14}$ Die gibt es übrigens wirklich, wenn auch zur Zeit der Abfassung dieses Textes noch in den Kinderschuhen; bemerkenswert dazu ist jedenfalls Chalmers 2017.
} 
partikuläre Interessen, etwa durch einzelwissenschaftlich bedingte methodische Einschränkungen, entwickelt wird. Der Ontologie geht es, wie gesagt, um das Existierende als solches, um das, was in den Praktiken als existierend vorausgesetzt wird, insofern es als existierend vorausgesetzt wird.

Dieser Allgemeinheitsaspekt besagt ferner, dass es in der Ontologie als Allgemeindisziplin nicht um die Summe einzelwissenschaftlicher Detailaspekte des Existierenden geht. Wer alle Ergebnisse einzelwissenschaftlicher Reflexionen kennen würde, wäre deshalb noch kein Ontologe/keine Ontologin. Allgemeinheit muss übrigens auch für regionale Ontologien gelten. Beispielsweise untersucht eine Ontologie der Biologie die Existenzvoraussetzungen der biologischen Praxis unter allgemeiner Rücksicht. Sie tut das nicht biologisch, sprich durch die Methodik einer empirischen Wissenschaft des Lebendigen eingeschränkt, sondern durch eine Analyse des biologisch Existierenden als Existierendem.

Damit können wir zu einem dritten Merkmal ontologischer Reflexion kommen: Ontologische Theorien sind integrativ. Mit dem Attribut ,integrativ“" werden ontologische Theorien - darin besteht ein Unterschied zu „universal" und ,allgemein" - nicht an sich, sondern in ihrem Verhältnis zu anderen Theorien gekennzeichnet, wobei trotz des Unterschieds dieser integrative Charakter in einem engen Zusammenhang mit den ersten beiden Merkmalen verstanden werden muss. ${ }^{15}$

Für eine Erläuterung von ,integrativ“ im hier intendierten Sinn muss die Annahme vorausgeschickt werden, dass Voraussetzungen bezüglich Existenz für jene Praktiken, in denen sie gemacht werden, relevant und die Praxis faktisch bestimmend sind. Existenzvoraussetzungen in der Mathematik, etwa bzgl. Zahlen, Mengen oder Klassen, beeinflussen die jeweilige Theorienbildung. Insofern es der Ontologie um die Klärung dieser Existenzvoraussetzungen geht, ist sie, in einer näher zu bestimmenden Rücksicht, für einzelwissenschaftliche Theorienbildung grundlegend. Die Ontologie integriert in ihrem Rahmen Grundlagen dieser Einzelwissenschaften. Der angesprochene inte-

${ }^{15}$ Diesen Gesichtspunkt hat Otto Muck als Eigenart der Metaphysik bzw. metaphysischer Theorienbildungen und Erklärungen hervorgehoben und ausführlich entwickelt, vgl. diverse Artikel in Muck 1999. 
grative Charakter hängt also wesentlich mit dem Programm einer Rahmentheorie zusammen.

Die Ontologie selbst hat übrigens keinen weiteren Rahmen. Sie hat keine Voraussetzungen, die außerhalb ihres eigenen Bereiches liegen. Sie kann nicht in irgendeinen anderen Kontext integriert werden. Unter Berücksichtigung dieses Gesichtspunkts wird auch verständlich, warum ontologische Rahmentheorien, insofern sie Theorien über Existierendes sind, auch als Theorien über die Grundstrukturen der Wirklichkeit bezeichnet werden.

Ergänzt wird dieser Aspekt noch dadurch, dass durch die Integration von einzelwissenschaftlichen Grundlagen in eine ontologische Rahmentheorie die Basis dieser Einzelwissenschaften nicht nur im Hinblick auf den Rahmen, sondern auch in Bezug auf andere Einzelwissenschaften in eine systematische Ordnung gebracht wird. Die Existenzvoraussetzungen beispielsweise von Physik und Mathematik können so in einen Zusammenhang gebracht werden, wohl ebenso wie jene von nicht-naturwissenschaftlichen Praktiken mit bestimmten naturwissenschaftlichen.

Mit diesen programmatischen Bemerkungen können wir zu einem Abschluss der Erläuterungen unserer Einstiegsdefinition von Ontologie kommen. Die Ontologie ist die systematische Reflexion auf jene Voraussetzungen bzgl. dessen, was ist oder existiert, wie sie in verschiedenen Praktiken gemacht werden, mit dem Ziel, eine konsistente Rahmentheorie über die Klärung dieser Voraussetzungen zu erlangen. „Systematisch“ bedeutet hier zunächst (ohne Ausschluss von Alternativen) analytisch-philosophisch vorzugehen, „Rahmentheorie" impliziert den universalen, allgemeinen und integrativen Charakter des anvisierten Entwurfs einer Ontologie.

Damit soll der Blick auch schon wieder auf unseren roten Faden zurïckgewendet werden, und das ist die aktuelle Metaontologie. In einem nächsten Schritt, sprich im zweiten Kapitel, sollen deshalb zunächst die KritikerInnen bzw. GegnerInnen des Projekts einer Ontologie zu Wort kommen. Wie angekündigt werden das AutorInnen in der Tradition Carnaps und Quines sein. Im kritischen Diskurs mit ihnen soll dann im dritten Kapitel der durch die Einstiegsdefinition gewonnene Ontologiebegriff weiterentwickelt werden in Richtung eines näheren Verstehens von Ontologie als Alltagsontologie. 


\section{Ontologiekritik in der aktuellen Metaontologie}

Standardeinwände gegen die Ontologie, wie sie in der aktuellen Metaontologie vorgetragen werden, können in drei Hauptstoßrichtungen eingeteilt werden. Da ist zunächst einmal Kritik unter dem Titel der Deflationierung: Ontologische Fragen seien kognitiv irrelevant. Letztlich bezögen sie sich auf Streite um Worte, die, wenn überhaupt, dann entweder rein nach pragmatischen Gesichtspunkten oder in einer sich der Trivialität annähernden Weise entschieden werden. Eine davon unterscheidbare, wenn auch mitunter mit der Deflationierung zusammenhängende Kritik kommt aus dem Naturalisierungsprogramm: Die wirklich interessanten Existenzfragen würden in den Naturwissenschaften entschieden. Ontologie sei somit entweder Teil der Naturwissenschaft, oder eine rein spekulative Tätigkeit ohne Bezug zur relevanten wissenschaftlichen Praxis. Im Hinblick auf die im letzten Kapitel versuchte Einstiegsdefinition könnte man die Essenz der naturalistischen Kritik auch so umschreiben, dass die naturwissenschaftliche Praxis, etwa die der Physik, im Hinblick auf Existenzvoraussetzungen grundlegend und für alle anderen Praktiken allein maßgeblich sei. Die Existenzannahmen von physikalischen Theorien seien letztlich entscheidend für die Erklärung der Grundstrukturen der Wirklichkeit. Eine dritte Stoßrichtung der GegnerInnen der Ontologie kann unter dem Vorwurf der Simplifizierung zusammengefasst werden. Im Anschluss an die naturalistische Kritik wird der Ontologie auch vorgeworfen, naturwissenschaftliche Forschungsergebnisse schlicht auszublenden, und somit, wenn überhaupt, dann nur ein unvollständiges, höchst vereinfachendes Bild der Wirklichkeit zu zeichnen. Damit geht der Vorwurf einher, an der Oberflächenstruktur alltäglicher Redeweisen hängen zu bleiben, und diese als vermeintliche Grundstrukturen der Wirklichkeit zu interpretieren. Die Ontologie sei letztlich naive Hypostasierung der Alltagssprache. Hier erkennen wir auch den Zusammenhang mit der DeflationierungsKritik: OntologInnen erkennen nicht, dass ihre Kontroversen eigentlich nur Dispute über die Bedeutung von sprachlichen Ausdrücken seien.

In einem ersten Schritt sollen diese Einwände etwas genauer aufgelistet und dargestellt werden, auch in ihrem inneren Zusammenhang. So weit je- 
denfalls, dass ihre Diskussion im Hinblick auf die weitere Entwicklung eines spezifischen Ontologiebegriffes (im dritten Kapitel) erfolgen kann. Dazu sollen die theoretischen Voraussetzungen der Kritiken ins Auge gefasst, aber auch ihre Reichweite analysiert werden. Was sind die genauen Referenten der jeweiligen Kritik, sprich ontologische Theorien, die tatsächlich von ihr betroffen wären? Ziel ist eine anfängliche Relativierung der Ontologiekritik in zwei Richtungen: Erstens ist natürlich auch diese Kritik, in den geschilderten Ausprägungen, nicht frei von (erheblichen, sich mitunter wechselseitig bedingenden) Voraussetzungen. Zweitens kann es keine apriorische Kritik an der Ontologie geben, sondern immer nur Gründe gegen bestimmte ontologische Theorienbildungen.

\subsection{Deflationierung}

Den einleitenden Überlegungen entsprechend können wir mit der unter dem Stichwort „Deflationierung“ vorgetragenen Kritik beginnen. Dabei kann es von Nutzen sein, zunächst den im Stichwort enthaltenen Begriff etwas näher zu betrachten.

Obwohl „Deflationierung“ auf einen im Englischen als philosophischer Fachterminus etablierten Begriff, nämlich „,deflationism“, zurückgeht, kann auch seine deutsche Ursprungsbedeutung aufschlussreich sein. Diese stammt aus der Wirtschaftsstatistik und meint die Bereinigung von in Geldeinheiten ausgedrückten Größen um den Einfluss der Inflation. Aus einer nominalen Größe wird durch Deflationierung eine reale. Im Kern steht also Bereinigung von vermeintlich Vorhandenem zu wirklich Vorliegendem. In der Philosophie hat sich Deflationierung bzw. der Deflationismus zunächst in der Wahrheitstheorie und in der Semantik als Position etabliert. Ersteres meint schlicht und einfach, dass ,wahr“ als Attribut von Sätzen verzichtbar sei. Zu behaupten, dass ein Satz wahr sei, hieße nichts anderes als den Satz selbst zu behaupten. ${ }^{16}$ Unser Sprachgebrauch, in diesem Fall in einer sehr speziellen Praxis, wird durch die Weglassung von nur vermeintlich sinnvollen Attributen bereinigt.

${ }^{16}$ Vgl. Stoljar/Damnjanovic 2014. 
In der Semantik kommt „deflationism“ v.a. in Referenzschemata zum Tragen. Was wir wirklich tun, wenn wir singuläre Ausdrücke referierend gebrauchen, ist nicht, uns auf bestimmte individuelle Gegenstände oder Referenten zu beziehen, auf deren Annahme wir uns dann auch ontologisch verpflichteten. Dass ein Sprecher/eine Sprecherin (S) auf einen Referenten (R) referiert, bedeutet ausschließlich, dass $\mathrm{S}$ etwas über $R$ sagt - unabhängig davon, ob sich $\mathrm{S}$ damit tatsächlich auf einen außersprachlichen Gegenstand $\mathrm{R}$ bezieht oder nicht. ${ }^{17}$ Der Referenz wird in diesem deflationierenden Schema jedenfalls die vermeintliche „Last der ontologischen Verpflichtung“ genommen; sie wird um diese Last bereinigt, könnte man in Anlehnung an die ursprüngliche Wortbedeutung von „Deflationierung“ auch sagen.

Damit kommen wir schon zum Wortgebrauch in dem für uns maßgeblichen Themenfeld, nämlich in der Metaontologie. Auch hier geht es im Kern um eine Bereinigung bzw. um Richtigstellung, die von einer vermeintlichen Aufgabe zu einer realen überführt. Die vermeintliche Aufgabe der Ontologie, welche VertreterInnen eines Deflationierungsprogramms bereinigen wollen, ist, dass es in der Ontologie um die Lösung theoretisch entscheidbarer Fragen über die Grundstrukturen der Wirklichkeit geht. VertreterInnen dieses Programms haben verschiedene Vorschläge entwickelt, worauf sich die „Scheinfragen“ der Ontologie nun tatsächlich bezögen. Der Radikalste ist, dass sich die Grundfragen der Ontologie als rein konzeptuelle Probleme erweisen ließen. Eigentlich gehe es ausschließlich um einen Streit um Worte, um es pointiert zu sagen. Weniger radikal anmutend, der Sache nach aber für die (traditionelle) Ontologie nicht minder bedrohlich, ist die Bereinigung ontologischer Probleme durch Überführung von vermeintlich ,schweren“, etwa, was es denn in der Welt (,,an sich“) nun wirklich gäbe, in real „leichte“, etwa, ob die Anwendungsbedingungen bestimmter Begriffe erfüllt seien oder nicht - was sich entweder logisch oder empirisch, jedenfalls spektakulär unspekulativ lösen lässt. Ontologie wird so zu Begriffsanalyse. Von einer „Easy Ontology“ zu unterscheiden ist der wohl anspruchsvollste Deflationierungsvorschlag: Zentrale Fragen nach der Existenz von Entitäten seien schlicht und einfach Fragen nach der Bedeutung von „Existenz“ bzw. des in der Prädikatenlogik verwendeten „Existenz“-Quantors. Wenn man versteht, dass „Exi-

${ }^{17}$ Båve 2009, 65. 
stenz" verschieden verwendet werden kann, lösen sich ontologische Probleme als Scheinprobleme auf. Quantorenvarianz ist die Lösung.

Im Wissen, dass damit sicherlich nicht alle vorliegenden Positionen angesprochen sind, geschweige denn weitere mögliche Ausformungen des Deflationierungsprogramms, und es auch innerhalb der drei erwähnten Auffassungen noch unterscheidbare Theorienbildungen geben mag, möchte ich mich zunächst der ersten Position zuwenden, welche die Ontologie insgesamt als Streit um Worte abtut.

\subsection{Ist Ontologie ein Streit um Worte?}

Dabei können wir uns zunächst nach Streitsituationen oder Disputen umsehen, die tatsächlich rein um Worte geführt werden. ${ }^{18}$ Das sind z.B. solche, die auf den Gebrauch derselben Wörter, genauer: von Vorkommnissen oder tokens desselben Worttyps, in verschiedenen Ideolekten zurückzuführen sind. Sind Fußbälle rund oder sind sie oval? ${ }^{19}$ Sollten sich zwei ExpertInnen über diese Frage tatsächlich in die Haare geraten, kann man ihnen durchaus nahelegen, zuvor zu überlegen, ob die eine mit „Fußball“ nicht das Spielgerät in einem klassisch europäischen Sport (soccer) meint, der andere jenes in einer außereuropäischen Abwandlung dieses bemerkenswerten Spiels (American football). Für eine weitere Möglichkeit verbaler Dispute erinnere ich mich an einen Aufenthalt auf der schönen Ostseeinsel Rügen. Ein Fremdenführer bot dort eine Wanderung auf einen Berg an. Es handelte sich um eine natürliche Formation, die sich alles in allem knappe 100 Meter über den Meeresspiegel erhob. Der Hinweis eines in Innsbruck lebenden Menschen, „Das ist kein Berg“, mündete tatsächlich in einen veritablen Disput. Hier könnte man sich freilich fragen, ob dies nicht ein Streit darüber ist, welche Objekte zur Extension des Wortes „Berg“ zu rechnen sind, ein Streit, der durch Begriffsklärung auch friedlich beigelegt werden könnte. Eine dritte Möglichkeit, die hier exemplarisch angeführt sei, geht auf Bedeutungsunterschiede durch Umstandsrelativität zurück. Streitet etwa ein Bewohner einer zentralsibirischen Provinz

\footnotetext{
${ }^{18}$ Ich beziehe mich hier auf die Charakterisierung von verbal disputes bei David Manley, in ders. 2009, 8-15.

${ }^{19}$ Aus Manley 2009, 8, übernommenes und adaptiertes Beispiel.
} 
mit einer Sizilianerin darüber, ob es in Innsbruck im Monat April in der Regel kühl sei, so kann dieser Streit wohl in Güte geschlichtet werden, wenn man die besagte Relativität des Attributs ,kühl“" mit in Betracht zieht. Für die eine ist eine Tagesmitteltemperatur von 15 Grad Celsius kühl, für den anderen nicht.

Alle drei Beispiele, die natürlich durch andere und auch anders gelagerte ergänzt werden könnten, haben gemeinsam, dass sich der Streit letztlich auf eine zunächst nicht offenkundige Differenz bzgl. der Bedeutung bestimmter sprachlicher Ausdrücke verstehen lässt. ,[...] speakers disagree because they semantically deviate from each other $[\ldots]^{، 20}$, wie Manley die Streit-um-Worte-Position auf den Punkt bringt. Ist die ,Semantik“ geklärt, sollten sich die Streitparteien in Einigkeit wiederfinden, und zwar so, dass keine Partei ihre ursprüngliche Position aufgeben müsste: Okay, Fußbälle sind oval, wenn du „Fußball“ im Kontext von American football, einem von meinem Sprachgebrauch zwar abweichenden, aber durchaus verträglichen Ideolekt, verwendest. Wenn du damit einverstanden bist, ist unser Streit beigelegt. Ja, wenn du „Berg“ nach Ostsee-Kriteriologie verstehst, sind wir uns einig: Dann fällt diese Erhebung unter den Begriff. Wenn du mir zugestehst, dass uns deine Kriteriologie in der alpinen Geographie nicht weiter bringt, und du mir meine Terminologie bei der Klassifizierung von Erhebungen in Tirol zugestehst, können wir es darauf beruhen lassen. Concedo, es ist kalt, wenn du „kalt“ relativ zu süditalienischen thermischen Verhältnissen verwendest. Von meinem sibirischen Kontext aus, lässt sich das gut zugestehen. Wenn dir diese Auflösung zusagt, brauchen wir nicht weiter zu diskutieren.

Wir halten fest: In diesen drei Beispielfällen wird bzw. würde der Disput durch Begriffsklärung beendet, wobei (i) ein/e OpponentIn den Sprachgebrauch der/des jeweils anderen, als auch (ii) dessen/deren inhaltlichen Standpunkt relativ zu diesem Sprachgebrauch, akzeptieren kann, ohne (iii) die eigene These aufgeben zu müssen.

In diesem Sinn soll zunächst eingeräumt werden, dass es Dispute geben mag, die auf den ersten Blick ontologisch anmuten, u.U. sogar von OntologInnen geführt werden, sich bei näherem Hinsehen aber tatsächlich in Analo-

${ }^{20}$ Ebd., 14. 
gie zu den angeführten Beispielen außerontologischer Auseinandersetzungen als Streit um Worte auflösen lassen.

Nehmen wir an, zwei KontrahentInnen debattieren darüber, ob es sich bei Eigenschaften um Adhärenzen, oder nicht doch um Momente handelte. Bei einer Klärung der Begriffe „Adhärenz“ und „Moment“ stellen sie fest, dass sie darunter Entitäten mit exakt denselben ontologischen Charakteristika meinen, etwa, dass diese partikulär sind, in einem strengen Sinn von ihren Trägern abhängen und den Trägern gewisse Qualitäten verleihen. Nachdem sie festhalten, dass sie aufgrund der terminologischen Festlegungen in ihrem theoretischen Rahmen bei ihren jeweiligen Begriffen bleiben wollen/sollen, können sie dennoch sowohl den Sprachgebrauch des anderen (siehe Streit-umWorte-Kriterium (i)) als auch den inhaltlichen Standpunkt relativ zu diesem Sprachgebrauch (siehe (ii)) akzeptieren, und zwar ohne eine eigene These aufgeben zu müssen (siehe (iii)). Damit wäre der Disput beigelegt, wohl in Analogie zum ideolektischen Beispiel mit den Bällen. Ein anderes Beispiel wären zwei KontrahentInnen, die sich darüber streiten, ob gewisse Prokaryoten, etwa Bakterien, Substanzen seien oder nicht. Bei einer Klärung des Begriffes stellen sie gewisse Abweichungen in ihrer Substanz-Kriteriologie fest, auf welche die Uneinigkeit in der Extension von „Substanz“ zurückzuführen ist. Allerdings ist diese Uneinigkeit so, dass die am Disput Beteiligten die Kriteriologie des/der jeweils anderen akzeptieren (siehe (i)) und die These des/der jeweils anderen relativ dazu für wahr halten könnten (ii), ohne, und das ist auch hier das Entscheidende, die eigene ontologische Position aufgeben zu müssen (iii). Dieser Disput wäre dann in genauer Analogie zur „Berg“-Extensions-Diskussion aufzulösen. Ein drittes Beispiel lässt sich ebenfalls an „Substanz“ darlegen. Nehmen wir eine Auseinandersetzung darüber, ob es sich bei einem bestimmten Quantum Salzsäure um eine Substanz handelt oder nicht. Eine Klärung der verwendeten Begriffe führt zur Feststellung, dass der eine „Substanz“ relativ zur Einführung in eine bestimmte philosophische Begrifflichkeit versteht, die andere aber in einem alltagssprachlichen bzw. populär-naturwissenschaftlichen Sinne. Auch hier ist es möglich, den Streit so beizulegen, dass man dem jeweils anderen sowohl seinen Sprachgebrauch zugesteht (i), als auch die Wahrheit seiner Aussage relativ zu diesem (ii), ohne dass einer der KontrahentInnen seine Meinung aufgeben 
müsste (iii). Hier würde ich eine klare Analogie zur Kontextrelativität beim „kühl“"-Beispiel sehen.

Die entscheidende Frage ist nun aber, ob sich sämtliche ontologischen Dispute auf diese Weise rekonstruieren und auflösen lassen. Dann hätte die Streit-um-Worte-Partei gegen die Ontologie gewonnen. Die folgenden Überlegungen richten sich gegen eine solche allgemeine Deflationierung ontologischer Probleme.

Als paradigmatisches Problem-Problem wird in der aktuellen metaontologischen Debatte gerne das spezielle Kompositionsproblem angeführt. Steht in diesem Raum ein Ding der Art Sessel, oder handelt es sich dabei, frei nach Peter van Inwagens berühmt-berüchtigter Theorie in Material Beings ${ }^{21}$, lediglich um sessel-weise arrangierte Atome oder simples? Anders gelagert ist das Problem, ob man die Fortdauer des Sessels durch die Zeit als Abfolge numerisch verschiedener raum-zeitlicher Sesselteile oder -phasen verstehen kann (im Sinne der Lewis'schen perdurance), oder ob der Sessel nicht doch als endurer in einem strikten Sinn durch die Zeit identisch fortbesteht? ${ }^{22}$ Kann man derlei Fragen im Sinne der Streit-um-Worte-Position auflösen? Das wäre dann der Fall, wenn man durch eine semantische Klärung der verwendeten Begriffe, etwa „Sessel“, „Atom“, „(diachrone) Identität“ bzw. „,raum-zeitlicher Teil" so weit zu einer Streitschlichtung käme, dass die eine Partei den Sprachgebrauch der jeweils anderen anerkennt (i), sowie die alternative Position als relativ zu diesem Sprachgebrauch zutreffend zugestehen könnte (ii), ohne dabei ihren eigenen inhaltlichen Standpunkt aufgeben zu müssen (iii).

Bleiben wir zunächst beim Streit um die Persistenz des Sessels. Können wir, z.B. aus der endurer-Position heraus, die Debatte so beenden, dass wir, im Sinne von (i), zugestehen: Okay, der Sessel ist nicht diachron identisch, wenn du „Identität durch die Zeit“" als komplexes, somit reduzierbares Faktum im Sinne der perdurance verstehst. Ja, es stimmt, der Sessel ist eine Summe raum-zeitlicher Teile, wenn du „raum-zeitlicher Teil“ nach Quine oder Lewis einführst. Concedo, die perdurance-Auffassung ist berechtigt, relativ zu einer prozessontologischen Einführung von „Persistenz“?

\footnotetext{
${ }^{21}$ Hier: van Inwagen 1990.

${ }^{22}$ Vgl. Lewis 1986, 202.
} 
Wenn nicht schon der erste, so spätestens der zweite kritische Blick zeigt, dass die semantische Klärung das eigentliche Problem, den Streit um Persistenz, nicht beseitigt. Die Akzeptanz des ,gegnerischen“ Sprachgebrauchs steht unter einer Bedingung, deren Erfüllung von weitreichenden theoretischen Zugeständnissen abhängt, in unserem Fall etwa, dass Identität durch die Zeit komplex und reduzierbar sei, dass Quines und Lewis' Einführung von raum-zeitlichen Teilen zutreffend wäre, dass man Persistenz prozessontologisch verstehen könne, und so weiter. Das aber sind genau jene Punkte, welche in der fraglichen Auseinandersetzung zur Diskussion stehen, und folglich vom Standpunkt der endurer-Partei nicht akzeptiert werden können.

Vergleichbares stellen wir bezüglich des Versuches fest, das spezielle Kompositionsproblem durch semantische Klärung der verwendeten Begriffe aufzulösen. Auch hier können wir Voraussetzungen formulieren, die geeignet sind, theoretische Rahmenbedingungen in den Blick zu bringen. Man kann die Rede über Sessel paraphrasieren in eine über sessel-weise arrangierte Atome, wenn die Einheits- und Kompositionsfrage für Artefakte im Allgemeinen nicht geklärt werden kann; bzw. wenn der Atomismus richtig liegt und wir es hier mit einem Agglomerat von „simples“ zu tun haben. Die „wenn dem so sein mag“-Klärung von van Inwagens Sprachgebrauch zeigt auch hier die Prämissen seiner Auffassung, in unserem Falle jener über Sessel und andere Artefakte. Sie machen den theoretischen Rahmen seines Sprachgebrauchs aus, der von einem Skeptiker seiner Auffassungen gerade nicht geteilt werden kann (gegen (i) bzw. (iii)), somit auch nicht einzelne Thesen relativ zu diesem Sprachgebrauch (gegen (ii)). Bei der Klärung der Begriffe endet der Streit also nicht, vielmehr beginnt er. Das ontologische Problem, ob Sessel nicht doch Grundelemente der Wirklichkeit sind, der Atomismus aber falsch ist, wird durch die semantische Analyse von „Sessel“ bzw. „Atom“ nicht gelöst, schon gar nicht aufgelöst.

Kurzum: Wenn man versucht, den Charakter von Streitigkeiten um Worte zu verstehen, kann man zum Ergebnis kommen, dass es ontologische Probleme, wie z.B. das spezielle Kompositionsproblem oder die Persistenz-Debatte gibt, die sich nicht als solche auffassen lassen. Der Versuch, diese Probleme als rein verbale Fragen darzustellen, scheitert und bestätigt ihre ontologische Relevanz. Das schließt, wie gesagt, nicht aus, dass es Dispute in der On- 
tologie geben mag, die sich tatsächlich als Streite um Worte auflösen lassen. Die hier vertretene Erwiderung gegen die Deflationierung der Ontologie unter diesem Vorzeichen ist somit bescheidener: Es gibt einen Korpus von Fragen, der sich nicht im Sinne der Streit-um-Worte-Position weginterpretieren lässt.

\subsection{Ist Ontologie ,easy“?}

Wir können uns damit einem weiteren aktuellen Vorschlag zuwenden, Ontologie bzw. ontologische Probleme zu deflationieren, in oben eingeführtem Sinne. Es ist die sogenannte Easy Ontology, wie sie federführend von Amie Thomasson vertreten wird. ${ }^{23}$ Die Ontologie soll von schweren Problemen bereinigt und in eine „leichte“ Lehre überführt werden, in deren Kern Begriffsanalyse als theoretisches Programm verbleibt. Da sich die „leichte Ontologie“ explizit als neo-carnapianisches Projekt versteht, soll sie zunächst auch von Carnap her eingeführt werden.

Carnaps Grundanliegen kann in dem nunmehr zur Debatte stehenden $\mathrm{Zu}$ sammenhang so umrissen werden, dass er die kognitive Relevanz der philosophischen Rede von Existenz, folglich auch von Existenzfragen, generell anzweifeln möchte. Der Ontologie geht es aber um Existenz und Existenzfragen. ${ }^{24}$ Also wird bei Carnap die kognitive Relevanz der Ontologie problematisiert. Carnaps Vorschlag in Güte besteht darin, die Ontologie von „harten“ oder „schweren“ dogmatischen Spekulationen über die Wirklichkeit (,an sich“) zu entbinden, und durch einen semantischen Aufstieg zur Klärung der Bedeutung von Existenzfragen zu kommen. Tun wir das, können wir zwischen sogenannten „externen“ und ,internen“ Fragen unterscheiden. In den einen werden bestimmte Begrifflichkeiten erwähnt, um für sie gewisse Verwendungsregeln festzulegen. Die anderen beruhen dann auf der konkreten Verwendung von Begriffen im Kontext festgelegter Terminologien. Ein bereits angeführtes Beispiel für Ersteres ist „Gibt es Zahlen?“. Nach Carnap geht es dabei keinesfalls um das alte platonische oder pythagoräische Rätsel

${ }^{23}$ Dabei ist v.a. ihr Buch Ontology Made Easy, hier Thomasson 2015, zu berücksichtigen. Zur Selbstcharakterisierung der Easy Ontology als deflationistisches Programm in der Metaon tologie siehe u.a. ebd., 22, 154.

${ }^{24}$ Thomasson 2015, 1. 
des Seinsstatus mathematischer Entitäten, sondern um die Anführung oder eben Erwähnung einer Sprachform, deren Akzeptanz allein nach pragmatischen Gesichtspunkten entschieden werden kann. Wird die jeweilige Sprachform, samt ihren Regeln, aber implementiert, können bestimmte Begriffe, entsprechend diesen Regeln, verwendet werden. Existenzfragen, die sich darauf beziehen, um beim gegebenen Beispiel zu bleiben „Gibt es Primzahlen größer als 100?“, sind dann logisch, also einfach zu entscheiden.

Die auf Carnap zu beziehende Unterscheidung zwischen Anführung oder Erwähnung (engl.: mentioning) und Verwendung (engl.: using) spielt nun auch in der Easy Ontology von Amie Thomasson eine entscheidende Rolle. Hier wird der eigentliche Sinn von ,schweren“ Debatten in der Ontologie darauf reduziert, die Frage nach der Erwähnung bzw. Einführung einer bestimmten Begrifflichkeit im Sinne von Carnaps externen Fragen zu klären. Die Frage beispielsweise, ob es endurer gibt, wäre dann als Problem anzugehen, ob sich pragmatische Gründe angeben lassen, einen begrifflichen Rahmen für durch die Zeit in einem strikten Sinn identische Gegenstände anzunehmen. ${ }^{25}$ Die Verwendungsregeln von Begriffen, wie z.B. „Sessel“, lassen sich aus den allgemeinen Regeln dieses Rahmens ableiten. Van Inwagens Frage, ob es denn überhaupt Sessel gebe, lässt sich dann leicht klären, zwar in diesem Falle nicht logisch, dafür aber empirisch. Wenn es in unserer Erfahrungswelt etwas gibt, das den besonderen Verwendungsregeln von „Sessel“ entspricht - Thomasson spricht in diesem Zusammenhang auch von Applikationsbedingungen, die mit dem Term verknüpft sind - dann (und nur dann) kann man davon sprechen, dass es eben Sessel gibt, dass Sessel existieren.

Der Hinweis auf Applikationsbedingungen führt uns von der grundlegend carnapianischen Ausrichtung der Easy Ontology zu einer etwas detaillierten Darstellung dieser Position.

Diese kann ansetzen bei der speziellen Quine-Kritik, die Thomasson zur Unterstützung ihrer Grundthese vorbringt. Quine sei, ihres Erachtens, ein Vorreiter einer dogmatischen oder ,schweren“ Metaphysik, insofern nämlich als er, die Arbeitsteilung zwischen Naturwissenschaften und Philosophie missachtend, nicht bereit ist, Begriffsanalyse als die eigentliche Aufgabe der

${ }^{25}$ Ebd., 40. 
Philosophie, somit auch der Ontologie, anzuerkennen. Naturalisiert man, wie Quine und seine SchülerInnen, die Ontologie, wird es ihr Geschäft, sich wie die Naturwissenschaften ,,[... in empirical enquiry aiming to discover matters of facts ${ }^{\text {‘26 }} \mathrm{zu}$ engagieren. Diese Suche nach Fakten (,,an sich“) sei aber der Beginn einer „schweren“ Metaphysik, der man nur durch Carnaps Rückführung der Ontologie auf Begriffsanalyse begegnen kann. Die Naturalismuskritik, die auf der Negierung einer Arbeitsteilung zwischen Philosophie und Naturwissenschaften aufbaut, kann bei Thomasson dadurch ergänzt werden, dass sie, gerade im Hinblick auf Existenzfragen, nicht nur jene ,ontologischen Verpflichtungen“ als maßgeblich erachtet, welche ,die besten wissenschaftlichen Theorien" eingehen, sondern auch jene, die wir in unserer alltäglichen Praxis machen. ${ }^{27}$ Ausgehend von unserem alltäglichen Reden lassen sich Analysen bezüglich der Verwendungsweise von Termini vornehmen, deren Regeln Aufschluss über Existenz und Existierendes geben.

Damit kommen wir wieder zurück zur bereits erwähnten Analyse von Applikations- oder Anwendungsbedingungen von Termini, einem Kernstück der Easy Ontology. Signifikanterweise werden bei Thomasson diese Applikationsbedingungen im Kontext der Darlegung einer Existenzbedingung eingeführt, die da lautet: „Ks exist iff the application conditions for , $\mathrm{K}^{\prime}$ are fulfilled“ ${ }^{28}$ Anwendungsbedingungen von Termini sind Bedingungen, deren Erfüllung Existenzannahmen in einem starken, sprich notwendigen und hinreichenden Sinn, legitimieren. Da an der angegebenen Stelle „K“ für Allgemeinbegriffe (nicht für Eigennamen) steht, können wir als erläuterndes Beispiel angeben, dass sich die Frage nach der Existenz etwa von Schafen zunächst darstellt als Frage, ob es denn etwas gibt, das den Applikations- oder Anwendungsbedingungen von "Schaf" entspricht. Die Antwort ist leicht, weil in Analogie zur Frage nach Sesseln, empirisch zu entscheiden. Damit wir das Schafexistenzproblem aber in dieser Weise als leichtes angehen kön-

\footnotetext{
${ }^{26}$ Ebd., 10.

${ }^{27}$ Diese Annahme wird von Thomasson in verschiedenen Kontexten ihres Buches hervorgehoben, siehe u.a. 20ff, 132, 322.

${ }^{28}$ Ebd., 86. Dort wird diese Existenzbedingung an der Stelle ,application conditions for , $\mathrm{K}^{\text {““ }}$ ergänzt durch ,,application conditions actually associated with , $\mathrm{K}$ “, um die Möglichkeit abzudecken, dass Ks auch dann existieren, wenn , $\mathrm{K}^{`}$ mit alternativen Applikationsbedingungen gekoppelt ist. Das spielt für unsere Darstellung jedoch keine Rolle.
} 
nen, müssen zuvor die begrifflichen Rahmenbedingungen geklärt werden, die zur Festlegung von Anwendungsbedingungen des fraglichen allgemeinen Terms, also von „Schaf“, führen. Das kann nur durch Anführung von Termini und ihrer externen Prüfung, im Sinne Carnaps, geschehen. So gesehen sind Existenzfragen eine spezielle Art der Verwendung von Termini, die zuvor eben angeführt und in ihren Verwendungs- oder Gebrauchsregeln geklärt worden sind. Dem entspricht die Ansicht, dass es sich bei Applikations- und Anwendungsregeln um „,basic rules of use“29, also um grundlegende Gebrauchsregeln handelt, die zuvor eingeführt und festgelegt werden müssen.

Die leichte Klärung von Existenzfragen kann im Kontext der Easy Ontology nicht nur durch die empirische bzw. logische Feststellung der Erfüllung von Anwendungsbedingungen erfolgen, sondern auch durch das, was bei Thomasson, wohl wieder in Anlehnung an Carnap, einfache Ableitungen (engl.: easy or simple inferences) genannt wird. Dabei beginnt man bei unkontroversen Aussagen, wie z.B. dass sich gleich viele Schafe wie Ziegen auf einem Weidegrund befinden. In trivialen Schritten, wie Thomasson auch sagt, ${ }^{30}$ wird daraus geschlossen, dass die Zahl der Schafe und die Zahl der Ziegen identisch ist, und daraus dann die ontologische Folgerung gezogen, dass es hier etwas gibt, das identisch ist, nämlich eine Zahl. Die Wahrheit des unkontroversen Ausgangssatzes garantiert, dass die Anwendungsbedingungen des neuen Terms (,Zahl“) erfüllt sind.

Die Frage, ob es denn Zahlen gibt, wird in diesem Sinne einfach, carnapianisch intern, gelöst. Im Anschluss an Carnap räumt Amie Thomasson aber ein, dass es PhilosophInnen, die sich die Frage nach der Existenz von Zahlen stellen, in der Regel nicht um derartige ,easy inferences“ geht. Normalerweise meinen sie damit eine externe Frage, die freilich - losgelöst von den internen Ableitungen - bei Carnap wie bei Thomasson ausschließlich pragmatisch, also nicht nach kognitiv relevanten Gesichtspunkten entschieden werden kann.

Vermutlich wird dieser Pragmatismus aber auch bei den easy inferences nicht auszublenden sein. Damit nämlich, wie beim gegebenen Beispiel deutlich wird, die einfachen Folgerungen funktionieren können bzw. sicherge-

\footnotetext{
${ }^{29}$ Ebd., 89.

${ }^{30}$ U.a. ebd., 21, 129-132.
} 
stellt werden kann, dass die Wahrheit des Ausgangssatzes die Erfüllung der Anwendungsbedingungen eines Terms garantiert, muss ja bereits ein bestimmter begrifflicher Rahmen festgelegt sein. In diesem Fall braucht es wohl Grundzüge einer Sprache, deren Regelwerk die Einführung von Zahlentermini mit besonderen Anwendungsbedingungen erlaubt. ${ }^{31}$ Das ist ja auch jene begriffliche Arbeit, nach pragmatischen Gesichtspunkten durchgeführt, die bei Carnap und Thomasson das Geschäft der Philosophie bleibt.

Mit diesem Hinweis auf die pragmatischen Voraussetzungen der simple inferences kommen wir auch schon zum Übergang vom Versuch einer Darstellung der Easy Ontology hin zu einer kritischen Erörterung, unter besonderer Rücksicht des hier relevanten Themas, nämlich Deflationierung.

Dabei können wir uns zunächst die grundsätzliche Frage stellen, gegen welche Ontologie die Theorienbildung der ,leichten Ontologie“ gerichtet ist, welche Art von Ontologie durch sie deflationiert werden soll. Eine erste Antwort wurde im darstellenden Teil bereits vorweggenommen. Thomasson richtet sich gegen das Projekt einer Naturalisierung der Ontologie, wie es federführend von Quine betrieben wurde. Wenn man die Ontologie gleichsam als Naturwissenschaft versteht, ignoriert man die Eigenart ontologischer Theorienbildung und läuft Gefahr, in ,schwere“ Spekulationen abzugleiten. Man könnte diese Kritik auch so verstehen, dass in naturalistischen Ontologien naturwissenschaftliche Erklärungsmodelle in unreflektierter Weise als Abbildungen von Tatsachen an sich aufgefasst werden. Das führt zu einer vorschnellen Ontologisierung oder Hypostasierung von Ergebnissen einzelwissenschaftlicher Theorienbildung. ${ }^{32}$ Fasst man jedoch die Ontologie als Grundlagenwissenschaft auf, die nach Maßgabe eigener Methodik die Voraussetzungen alltäglicher wie wissenschaftlicher Praxis hinsichtlich Existenz und Existierendem reflektiert, ist man unter dieser Rücksicht nicht im Fokus der Deflationierungsbestrebungen der Easy Ontology.

\footnotetext{
${ }^{31}$ Ebd., 129, spricht Thomasson davon, dass es für easy inferences kompetente SprecherInnen (engl.: competent speakers) braucht, die gemäß eingeführten Regeln bereits Applikationsbedingungen von Termini beherrschen.

${ }^{32}$ Diesen Gesichtspunkt habe ich versucht in Kanzian 2016, Abschnitt I - 2.2.2, auszufalten. Er wird auch im Folgenden eine Rolle spielen. Er kann daher in dem nunmehr relevanten Zusammenhang, das ist die Easy Ontology, kurz gehalten werden.
} 
Thomasson richtet sich natürlich nicht nur gegen naturalistische Ontologie. Ihr Anliegen kann, positiv gefasst, auch so interpretiert werden, dass sie gegen Ansätze naiver Ontologie auftritt. Naiv wäre es etwa, ohne vorherige Prüfung begrifflicher Voraussetzungen die Grundstrukturen der Wirklichkeit (,,an sich“) angehen zu wollen. Dem entspräche eine extreme, sprich unkritische Spielart des Realismus. Es kann jetzt nicht die Aufgabe sein, historisch zu untersuchen, welche Positionen in der älteren oder auch in der aktuellen Ontologie unter ein derartiges Verdikt fallen würden. Hier geht es nur darum festzuhalten, dass dies keinesfalls jede ontologische Theorienbildung betrifft: eine Ontologie, die ihre Reflexion im Anschluss an eine Analyse alltäglicher oder wissenschaftlicher (Sprech-)Praxis vornimmt, wohl nicht.

Eine weitere Ebene der Auseinandersetzung könnte darin bestehen zu fragen, ob die begriffliche Analyse, in der Weise, wie sie die Easy Ontology propagiert, tatsächlich in der Lage ist, die Ontologie in der Vielfalt ihrer Aufgaben abzudecken. Diese kritische Anfrage zielt zum einen darauf ab, ob die Klärung von Existenzfragen tatsächlich so „easy“ ist, wie Thomasson das vorschwebt; zum anderen aber darauf, ob mit der Klärung von Existenzfragen tatsächlich schon das gesamte Geschäft der Ontologie erledigt ist.

Bleiben wir zunächst beim Ersten. Hier ist Amie Thomasson explizit, indem sie es ablehnt, über die Erfüllung von Anwendungsbedingungen von Termini hinausgehende Existenzkriterien in Betracht zu ziehen. In diesem Zusammenhang wendet sie sich beispielsweise gegen das Kriterium der kausalen Wirksamkeit (engl.: causal efficacy) oder der Bewusstseinsunabhängigkeit (engl.: mind-independence) oder andere substantielle (engl.: substantive) Kriterien zur Klärung von Existenzfragen. ${ }^{33}$ Ihre Argumente können so auf den Punkt gebracht werden, dass (i) Existenz keine ,tiefe und substantielle Natur" habe. ${ }^{34}$ Dementsprechend sei eben ein rein formales Kriterium wie das in der Easy Ontology gegebene adäquat, nicht irgendwelche inhaltlichmaterialen. Thomasson argumentiert weiter (ii), dass verschiedene Typen oder Kategorien von Entitäten verschiedene Existenzkriterien bräuchten. Dementsprechend könnten weder kausale Effizienz noch Bewusstseinsunabhängigkeit, weil allgemein, als Existenzkriterien fungieren. Schließlich (iii)

\footnotetext{
${ }^{33}$ Thomasson 2015, 81, 89, $115 \mathrm{ff}$.

${ }^{34}$ Ebd., 116 f.
} 
könnten Entitäten nach den „easy criteria“ auch dann als existent ausgewiesen werden, wenn sie die „schweren“ Kriterien nicht erfüllen. Letztere können somit nicht notwendig sein.

Hier soll es nicht darum gehen, eines der von Thomasson zurückgewiesenen Existenzkriterien zu verteidigen. Bewusstseinsunabhängigkeit ist als allgemeines Existenzkriterium wohl ebenso wenig hinnehmbar, ${ }^{35}$ wie es kausale Effizienz ist, insbesondere wenn diese auf engführende Konzepte von Kausalität und kausaler Wirksamkeit rekurrieren. Die Äußerung des Verdachts, dass Thomasson bei ihrer Argumentation gegen substantielle Existenzkriterien das „leichte“ Verständnis von Existenz bereits voraussetzt, insbesondere bei (i) und (iii), sei aber dennoch gestattet. Besagte Argumentation kann also bestenfalls eine Anwendung der Prämissen der Easy Ontology bzgl. des Verstehens von Existenz auf das Thema Existenzkriterien sein. Sie leistet aber keine zusätzliche Unterstützung der Grundthesen der Easy Ontology; insbesondere wenn man bedenkt, dass die verbleibende Argumentationslinie (ii) nicht gegen ,schwere“ oder substantielle Existenzkriterien spricht, sondern nur dagegen, dass es für alle angenommenen Entitäten ein einziges gäbe. Das aber ist eine Ansicht, die auch von OntologInnen geteilt werden kann, welche ihre Disziplin nicht im Sinne der Easy Ontology deflationieren.

Wir können uns damit der zweiten angedeuteten Frageebene zuwenden, ob denn mit der Klärung von Existenzfragen tatsächlich schon das gesamte Geschäft unserer Disziplin erledigt sei. Hier finden sich bei Thomasson Ansätze, die eine differenzierende Antwort nahelegen. So stellt sie es durchaus in Frage, ob sich beispielsweise das Thema Modalitäten ebenso „leicht“ abhandeln lässt, wie Lösungen im Zusammenhang mit Existenz. Ebenso verhält es sich mit dem Problem, ob wir ein Viel-Schichten-Modell der Wirklichkeit annehmen sollten, oder vielleicht doch besser eine einzige Schicht oder Ebene. Neigen wir zu Ersterem, stellt sich die Frage nach einer fundamentalen Ebene bzw. nach dem Verhältnis der verschiedenen Ebenen. Helfen hier Konstitution, gar Supervenienz? ${ }^{36}$ Nehmen wir nur eine Ebene der Wirklichkeit an, bleibt zu klären, was bzw. welche wir als solche akzeptieren? Unab-

\footnotetext{
${ }^{35}$ Dies habe ich in Kanzian 2009, 109ff, im Kontext einer Zurückweisung von Argumenten gegen Artefakte ausgeführt.

${ }^{36} \mathrm{Vgl}$. Thomasson 2015, 325.
} 
hängig von einer Antwort versucht Thomasson die Strategie, all diese Themenstellungen als solche einer Metaphysik zu deklarieren, wohl, um sie die Leichtigkeit der Ontologie nicht gefährden zu lassen. Dem soll nicht die prinzipielle Ungangbarkeit einer Unterscheidung zwischen Metaphysik und Ontologie entgegengehalten werden. Das wäre sowohl historisch falsch als auch sachlich kurzschlüssig. Dennoch kann kritisch vermerkt werden, dass die von Thomasson anvisierte Grenzziehung an dieser Stelle doch etwas willkürlich anmutet. Und zwar deshalb, weil offensichtlich all die von ihr angeschnittenen „metaphysischen“ Probleme unmittelbar mit der Existenz von Entitäten, dem verbleibenden Betätigungsfeld ihrer Ontologie, zu tun haben. Es ist ja nicht nur so, dass die Klärung von Existenzfragen im Allgemeinen mit der Annahme von Schichten der Wirklichkeit einhergeht. Auch die Akzeptanz eines konkreten kategorialen Rahmens hängt intrinsisch damit zusammen, ob wir etwa die Ebene der Mikrophysik als die fundamentale Schicht der Wirklichkeit annehmen oder aber unsere alltägliche Lebenswelt. Während sich für den ersteren Zugang ein prozess- oder tropenontologisches Kategorienschema nahelegt, wird man für den letzteren auf ding- oder substanzontologische Interpretationen zurückgreifen.

Vielleicht könnte man in diesem Zusammenhang die doch nicht nur „leichte“, sondern auch leichtfertige Einführung von Kategorien durch die Easy Ontology - so werden Applikationsbedingungen von nach pragmatischen Gesichtspunkten eingeführten Terminologien mitunter in recht inflationärem Ausmaß erfüllt - kritisieren. Und zwar so, dass sie eben losgelöst von den angeführten ,schweren“ Themen erfolgt, ohne dass dies weiter hinterfragt würde.

Zusammenfassend können wir festhalten: Das Deflationierungsvorhaben der Easy Ontology betrifft nicht die Ontologie als ganze, sondern bestimmte (naive) Varianten ontologischer Theorienbildung. Darüber hinaus lässt sich zeigen, dass die ,leichte“ Deflationierung auf bestimmten Annahmen inhaltlicher und ontologie-theoretischer Art beruht, welche ihrerseits aus naheliegenden Gründen in Frage gestellt werden können. Auch der Deflationierung der Easy Ontology ist die Ontologie also nicht hilflos ausgesetzt. 


\subsection{Quantorenvarianz}

Wir können damit zu einem weiteren Projekt kommen, das in der Literatur als Deflationierungsversuch der Ontologie vermerkt wird. Es geht von der Annahme aus, dass der Existenzquantor nicht eine von vornherein festgelegte Bedeutung hat, sondern semantisch variieren kann. Insofern es der Ontologie um die Klärung von Existenzfragen geht, sich aber Existenzfragen, frei nach Quine, als Fragen nach dem Wertebereich von durch Quantoren gebundenen Variablen auffassen lassen, ist die Relevanz der Ontologie durch diese Lehre von der Quantorenvarianz in ihrer Substanz betroffen. In ihrer Bedeutung variierende Quantoren bzw. die durch sie gebundenen Variablen haben nämlich unterschiedliche Wertebereiche. Wenn dem so ist, lassen sich ontologische Streitigkeiten relativieren, bzw. durch semantische Analysen, nämlich von angenommenen Bedeutungen von Quantoren, auflösen. Man muss dann z.B. nicht mehr darüber streiten, ob die Dinge unserer Alltagswelt endurer oder perdurer sind. Es reicht die Annahme, dass es eben unterschiedliche Existenzquantoren gibt: Der eine läuft über endurer, der andere über Lewis' dingliche Phasen und deren Summen.

Dieser Ansatz zur Deflationierung der Ontologie kann durchaus als eine technisch anspruchsvollere Fortführung der Streit-um-Worte-Position angesehen werden. Wenn sich, um beim Beispiel zu bleiben, die Debatte, ob diachrone Identität im Sinne der endurance (E) oder der perdurance $(\mathrm{P}) \mathrm{zu}$ verstehen ist, auflösen lässt als Streit über die Adäquatheit einer E-Sprache bzw. einer P-Sprache, in der eben die Quantoren E-spezifische bzw. P-spezifische Bedeutung haben, reduziert sich eine Kernfrage der aktuelleren Ontologie auf einen Streit über Sprachen oder Sprechweisen, wenn man so will, eben über Worte.

Anschluss kann die Lehre von der Quantorenvarianz auch an die bereits behandelte Easy Ontology finden, insofern sich namhafte Vertreter, wie Eli Hirsch, gegen eine ,hypertheoretische“ Ontologie (engl.: hyper-theoretical-

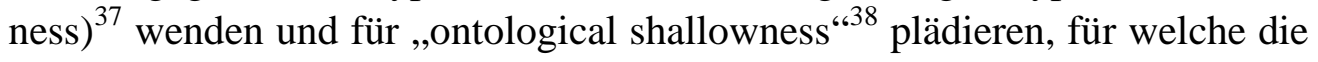
„Leichtigkeit“" von Amie Thomasson noch eine ontologie-wohlwollende

${ }^{37}$ Hirsch 2002, 61.

${ }^{38}$ Ebd., 63. 
Übersetzung darstellte. Jedenfalls werden divergierende ontologische Standpunkte, relativ zur Einführung von bedeutungsunterschiedlichen Quantoren, leicht vertretbar und schwer angreifbar. Eine Explikation von Existenzannahmen, relativ zu Sprechweisen mit entsprechenden semantischen Regeln, wird jedenfalls zu einem recht seichten Geschäft.

Um allerdings die Lehre von der Quantorenvarianz in ihrer reifsten Form, das ist wohl jene, die ihr Hirsch gegeben hat, richtig einordnen zu können, muss man sie auch von gewissen Extremvarianten unterscheiden. Als solche könnte man zum einen Ryles ontologischen Pluralismus verstehen, zum anderen Putnams Anti-Realismus.

Schon nach Ryle gibt es keine einheitliche Bedeutung von „existieren“ bzw. „es gibt“ “ ${ }^{39}$ sondern je nach Gegenstandsbereich sehr unterschiedliche. So behauptet man schlichtweg Verschiedenes, wenn man aussagt, dass Sessel existieren, und wenn man davon spricht, dass Zahlen existieren. Die Bedeutung von ,existieren“ in diesen Beispielfällen ist ebenso verschieden und unvereinbar wie die Bedeutungen von „Bank“ im Fall von Sitzgelegenheiten in Parks und von „Bank“ im Sinn von Geldinstituten. „Existieren“ und „Bank“ in beiden Fällen sind glatte Äquivokationen. Es gibt nach Ryle somit nicht einen Existenzquantor, sondern deren viele, ohne dass wir in dieser Vielfalt eine verbindende Einheit in ihrer Bedeutung feststellen könnten. Existenzfragen verlieren damit jedwede kognitive Relevanz. Fragen wir uns, ob Zahlen existieren, kann es darauf keine nicht-triviale Antwort geben: Natürlich existieren Zahlen, wenn wir eine zahlen-freundliche Semantik des Existenzquantors zulassen.

Hirsch kann man nicht in Richtung dieser glatten Äquivokation interpretieren. Die möglichen Bedeutungsvariationen des Existenzquantors lassen sich bei ihm vermitteln. Es ist durchaus möglich, semantische Brücken zwischen verschiedenen Quantoren, etwa den erwähnten E- und P-Quantoren, zu schlagen. Diese Brücken sind sogar so stabil, dass sich beispielsweise in der E-Sprache eine Semantik der P-Sprache darlegen lässt. ${ }^{40}$ Hirsch vertritt auch keinen undifferenziert egalitären Pluralismus. Nach ihm gibt es durchaus eine privilegierte Weise der Quantorenverwendung. Es ist, um es an dieser Stelle

\footnotetext{
${ }^{39}$ Vgl. Ryle 1949, 23.

${ }^{40}$ Hirsch 2009, 245.
} 
vorwegzunehmen, jene, die sich an unserem alltäglichen Sprachgebrauch orientiert.

Nicht gegen Ryle, aber gegen Putnams Anti-Realismus richtet sich Hirschs These, dass sich aus der Verschiedenheit der Bedeutungen des Existenzquantors keinesfalls die Sprachabhängigkeit dessen, was existiert, folgern lässt. ${ }^{41}$ Dies erscheint umso relevanter, als sich Putnam in einschlägigen Arbeiten als richtungsweisender Vertreter der Quantorenvarianz hervorgetan und genau das mitbehauptet hat. Der springende Punkt besteht für Hirsch darin, dass wir uns mit Festlegungen bezüglich der Bedeutung des Existenzquantors, somit der Bedeutung von „Es gibt ..."-Aussagen, natürlich im Bereich von „linguistic decisions“ bewegen. ${ }^{42}$ Ebenso ist es Sache sprachlicher Bestimmungen, entsprechende semantische Regeln $\mathrm{zu}$ formulieren, damit auch die Wahrheit und Falschheit von „Es gibt ..."-Aussagen festzulegen. So hängt es von Entscheidungen über die Bedeutung von „Es gibt ...“ ab, ob wir für die Aussage „Es gibt Sessel“ den Wahrheitswert wahr oder den Wahrheitswert falsch annehmen müssen. Es wäre allerdings irrig zu meinen, so Hirsch, dass sich an dieser Festlegung semantischer Konventionen die Existenz von Sesseln entscheidet. Das wäre ,,an absurd form of linguistic idealism that is not at all implied by quantifier variance ${ }^{643}$, welche letztlich auf eine Verwechslung der Anführung (engl.: mentioning) und der Verwendung (engl.: using) sprachlicher Ausdrücke zurückzuführen ist. Von der Anführung von ,Es gibt ...“, in der man auch über seine Bedeutung reflektieren mag, ist eben seine Verwendung zu unterscheiden, welche außersprachliche Existenz bzw. Nicht-Existenz meint. Bedeutungsfestlegungen sind sprachabhängig, Existierendes nicht.

Diese anti-idealistische Tendenz ist ein wesentlicher Aspekt von Eli Hirschs Theorie der Quantorenvarianz. Weitere Charakterisierungen können unmittelbar daran anknüpfen. Eine davon besteht in einem vertiefenden Blick auf das Verhältnis zweier unterschiedlicher Quantoren-Bedeutungen, deren Differenz so gravierend ist, dass sie zu unterschiedlichen Wahrheitswerten

${ }^{41}$ Siehe die programmatische Ansage im Titel von Hirsch 2002 ,Quantifier Variance and Realism“.

${ }^{42}$ Hirsch 2002, 52.

${ }^{43}$ Ebd. 
von „Es gibt...“-Aussagen führt. Bleiben wir beispielhaft bei den oben bereits erwähnten E- und P-Quantoren, deren Semantik im Kontext einer endurer-Sprache (E), welche die Existenz zeitlicher Teile von alltäglichen Dingen negiert, bzw. einer alternativen perdurer-Sprache $(\mathrm{P})$, festgelegt ist. Dieses Verhältnis ist nach Hirsch so zu interpretieren, dass sich in E weitgehende Konzessionen im Hinblick auf $\mathrm{P}$ formulieren lassen. So könnte man etwa in E einräumen: Über die Persistenz von alltäglichen Dingen können wir reden, als $o b$ (engl.: as if) sie in der Kontinuität numerisch verschiedener zeitlicher Phasen bestünde, wenn man die Bedeutung des Existenzquantors im Sinne von $\mathrm{P}$ festlegt. Die Möglichkeit eines solchen entgegenkommenden Vergleiches (engl.: simile) zwischen E und P beruht auf der Annahme einer vergleichbaren Quantorenbedeutung in diesen Sprachen. Dieser Vergleich birgt freilich auch ein Risiko in sich. Dieses besteht darin, das ,als ob“ unter den Tisch fallen zu lassen, und durch eine verkürzende Formel zu ersetzen: Zeitliche Phasen gibt es, relativ zu P. Wenn man diese verkürzende Formel als Metapher für die längere Als-ob-Wendung ansieht, mag das noch hinzunehmen sein. Löst man sie jedoch los vom ursprünglichen Kontext, landet man in „lunacy“ und „madness““. 44 Dann behauptet man nämlich, dass die Existenz zeitlicher Teile tatsächlich von semantischen Regeln, nämlich jenen, welche in P gelten, abhängig ist. Wir landeten in dem von Eli Hirsch kritisierten linguistischen Idealismus.

Der Hinweis auf die besondere Relevanz der Als-ob-Klausel und der mit ihr einhergehenden „Wenn-Bestimmung“, lässt sich bei Hirsch auch dadurch ergänzen, dass er davor warnt, mögliche Quantorenvarianz mit einer faktischen zu verwechseln, worin Hirsch auch einen Unterschied seiner Variante der Quantorenvarianz zu jener von Putnam festmacht. ${ }^{45}$ Im Hintergrund steht die Überzeugung, dass es, vom Standpunkt einer E-Sprache, durchaus möglich ist, relativ zu bestimmten theoretischen Interessen, den EQuantor in seiner Bedeutung zu modifizieren, etwa zu einem P-Quantor. Dem entspricht die Auffassung, dass die Rede von zeitlichen Teilen von alltäglichen Dingen durchaus ihren Sinn haben mag, semantisch gesprochen, mit positiven Wahrheitswerten ausgestattet werden kann. Wenn man etwa

\footnotetext{
${ }^{44}$ Hirsch 2002, 55.

${ }^{45}$ Vgl. Hirsch 2002, 60.
} 
Alltagsdinge in physikalischen Modellen, welche eine vierdimensionale Raum-Zeit bedingen, rekonstruieren möchte. Daraus folgt aber nicht, und das ist der entscheidende Punkt, dass wir die faktische Verwendung des Existenzquantors verändern. Durch eine modellhafte Darstellung von Alltagsdingen in physikalischen Theorien machen wir sie nicht zu vierdimensionalen RaumZeit-Zonen.

Ein Vorteil dieses undogmatischen Verstehens von Quantoren besteht für Hirsch darin, dass sich schwere ontologische Diskussionen semantisch rekonstruieren und auch auflösen lassen. Als EndurantistInnen brauchen wir nicht weiter auf der Falschheit der Zeitlichen-Teile-Rede bei Dingen beharren. Wir können einräumen, dass PerdurantistInnen, aufgrund berechtigter theoretischer Interessen, von einer legitimen Variation der Bedeutung des Existenzquantors Gebrauch machen. ${ }^{46}$ Relativ zu einer solchen P-Variation wird die Annahme von zeitlichen Dingteilen trivialerweise wahr. Diesen Vorteil der Auflösung eines ontologischen Kernproblems können wir genießen, wenn wir auf eine metaphysisch privilegierte Quantorenbedeutung, Hirsch nennt diese leicht mißverständlich auch „God's quantifier“47, verzichten, folglich auf hyper-theoreticalness in der Ontologie. Wir müssen, selbst als eingefleischte EndurantistInnen, nicht mehr mit allen zur Verfügung stehenden theoretischen Mitteln auf den Perdurantismus losgehen. Es reicht der Hinweis, dass PerdurantistInnen eben im Kontext ihrer P-Sprache, entsprechend ihrer P-Interessen, eine P-relative Quantorenbedeutung angenommen haben, welche ihre Rede über zeitliche Dingteile trivialerweise wahr macht.

Plädiert Eli Hirsch damit für einen ,anything goes“ Standpunkt in ontologischen Fragen? Nein! Das wird aus den bereits geschilderten anti-idealistischen Grundannahmen und den sich daraus ergebenden Distinktionen klar. Wenn es auch keine, aus metaphysischen Gründen (!) privilegierte Quantorenbedeutung gibt, so doch eine faktische Grund- bzw. Ausgangsbedeutung des Existenzquantors. Diese entspricht der aktuellen Verwendung von „Es gibt ..." in unserer alltäglichen Sprache; Hirsch spricht in diesem Zusammenhang auch von ,plain English“ ${ }^{48}$ Hirsch geht also von einer faktischen oder,

\footnotetext{
${ }^{46}$ Vgl. Hirsch 2002, 63.

${ }^{47}$ Ebd.

${ }^{48}$ Hirsch 2002, 60.
} 
wenn man möchte, pragmatischen Priorität des „,common sense“ in Existenzfragen aus. ${ }^{49}$ Von hier aus werden im Sinne der Als-ob-Klausel auch andere Bedeutungen des Existenzquantors möglich. Wir können so sprechen, als $o b$ es zeitliche Teile von Dingen gibt. In diesem Sinn können wir auch Sprachen entwickeln, in denen die Behauptung beliebiger Dingkompositionen als wahr, die Annahme der Existenz etwa von alltäglichen Gebrauchsgegenständen wie Sessel hingegen als falsch zu erweisen sind. Wichtig ist nur, dass wir nicht meinen, damit die Welt zu verändern. Wir verändern die Bedeutung von „Existenz“ und damit die Interpretation des Streites darüber, was es denn in der Welt nun wirklich gibt, oder nicht.

Aus diesen Überlegungen ergibt sich die Möglichkeit einer differenzierenden Sichtweise auf das Thema Quantorenvarianz und Deflation der Ontologie. Zunächst kann in diesem Zusammenhang durchaus zugestanden werden, dass es Dispute geben mag, die sich ohne tiefergehende Debatten im Sinne einer Quantorenvarianz auflösen lassen. Das wären etwa solche, in denen die Streitparteien bedeutungsunterschiedliche Quantoren verwenden, allerdings so, dass sie in der Lage sind, die Sprachform der alternativen Partei anzuerkennen, samt der Wahrheit der von den „GegnerInnen“ formulierten Thesen relativ zu deren Sprachform, ohne die eigene Position aufgeben zu müssen.

Im Anschluss an Eli Hirsch können wir allerdings auch klare Grenzen gegenüber Versuchen ziehen, ontologische Fragen generell zu deflationieren. Das ergibt sich schon daraus, dass Hirsch auch ontologische Dispute einräumt, die sich nicht rekonstruieren lassen als durch unterschiedliche Bedeutungen von Quantoren bedingt. Dementsprechend gibt es auch keine irenische Schlichtung des Streits aufgrund einer Darstellung der alternativen Position im Sinne der eben besprochenen ,,as if“-Klausel. Explizit spricht Hirsch in diesem Zusammenhang den Streit um die Existenz abstrakter Entitäten an. Hirsch negiert, dass man, etwa vom Standpunkt des Nominalismus aus, eine platonistische Sprache akzeptieren kann, in der ein spezieller A-Quantor, der über abstrakte Entitäten laufen mag, eingeführt wird und der Rede über Abstrakta (trivialerweise) positive Wahrheitswerte zukommen lässt. Das funktioniert ebenso wenig wie das Umgekehrte, die Akzeptanz eines nominalisti-

${ }^{49}$ Ebd., 60-63. 
schen Quantors durch die abstrakta-freundliche Partei. ${ }^{50}$ Wenn wir die im Streit-um-Worte-Abschnitt vorgeschlagene Kriteriologie aufgreifen, können wir das so verstehen, dass es vom nominalistischen Standpunkt aus schlicht nicht möglich ist, einen platonistischen Sprachgebrauch zuzulassen, dazu die Wahrheit einer abstrakta-freundlichen Position relativ zu diesem Sprachgebrauch, ohne - und das ist entscheidend - den eigenen nominalistischen Standpunkt aufgeben zu müssen. Gesteht man theoretische Erfordernisse zu, welche die Einführung eines A-Quantors bedingen, kann man nicht weiter NominalistIn sein. Aus analogen Gründen kann es auch keine rein nominalistische Sprache vor einem platonistischen Hintergrund geben. Es bleibt uns OntologInnen also nichts anderes übrig als weiter darüber zu streiten, welche Position nun tatsächlich die adäquate ist: Nominalismus oder Platonismus. Wenn dem so ist, haben wir jedenfalls ein starkes Argument dagegen in der Hand, dass die Ontologie insgesamt obsolet sei im Sinne eines sich auf Quantorenvarianz berufenden Deflationierungsprogramms.

Um zu diesem Ergebnis zu kommen, können wir aber auch auf jene Streitfälle Bezug nehmen, von denen Hirsch meint, dass sie durch den Hinweis auf unterschiedliche Bedeutungen der von den Parteien verwendeten Quantoren beizulegen wären, allen voran den Disput zwischen EndurantistInnen und PerdurantistInnen. Hirsch ist ja davon überzeugt, dass es von einem endurantistischen Standpunkt aus möglich ist, relativ zu bestimmten theoretischen Zielsetzungen, den E-Quantor in seiner Bedeutung zu modifizieren, etwa zu einem P-Quantor. So gesehen, kann die Endurantistin so sprechen, als $o b$ die vom Perdurantisten gewählte Sprachform wahre Aussagen beinhaltete. Tatsächlich muss der Endurantist seinen Standpunkt nicht aufgeben, wenn er die perdurantistische Sprache, nach Einräumung bestimmter theoretischer Interessen - siehe oben, zulässt, und somit in $P$ auch wahrheitsfähige Aussagen. Es sei jedoch auch darauf hingewiesen, dass dies nicht jeden ontologischen Streit erübrigt. Man könnte sich nämlich durchaus darüber auseinandersetzen, welche Sprache nun die primäre ist, welche aber jene, die, durch gewisse eingeschränkte Interessen erzeugt, Sätze beinhaltet, die man semantisch bewerten kann, als ob sie wahr wären. Ist die P-Sprache grundlegend, und kann man aus ihrer Perspektive die E-Sprache als Alternative ansehen,

${ }^{50}$ Vgl. dazu u.a. Hirsch 2009, $252 \mathrm{ff}$. 
die man relativ zu bestimmten Zielsetzungen als wahrheitsfähig erachtet; oder ist es die E-Sprache, von der ausgehend man die P-Sprache einführen kann? Dass hier eine Entscheidung fällig ist, entspricht Hirschs Annahme, dass es faktisch eine Grund- oder Ausgangsbedeutung des Existenzquantors gibt, aus der heraus sich die Alternativen erst rekonstruieren und verstehen lassen. Welche das aber ist, führt zu einer Auseinandersetzung, die ontologischer Argumentation bedarf. Im Falle der Alternative E-Sprache/P-Sprache ist das offensichtlich. Der Standpunkt, dass es sich bei der E-Sprache um die grundlegende Sprachform handelt, entspricht einer alltagsontologischen $\mathrm{Zu}$ gangsweise; die Alternative aber einer Ontologie, welche geneigt ist, physikalische Modelle zum Ausgang ihrer Theorienbildung anzunehmen. Wer Recht hat, muss sich in einem nicht zu deflationierenden Disput herausstellen, in dem grundlegende Einstellungen zu den Grundstrukturen der Wirklichkeit eingebracht werden müssen.

Jedenfalls ist die Annahme der Quantorenvarianz nicht geeignet, die Ontologie als philosophische Disziplin zu deflationieren. Diese Gründe finden sich, wie gesehen, z.T. bei Hirsch, z.T. lassen sie sich aus den Thesen Hirschs herleiten.

Zum Abschluss soll nun auch noch auf solche Gründe gegen den Erfolg des Deflationierungsprogramms hingewiesen werden, die über die Theorienbildung bei Hirsch hinausgehen, bzw. sich sogar gegen dieselbe richten. Um bei Letzterem zu beginnen, kann darauf hingewiesen werden, dass bei Hirsch die Festlegung auf eine grundlegende Sprachform, etwa die Alltagssprache, damit verbunden auf eine eigentliche Bedeutung des Existenzquantors, selbst nicht weiter zu begründen ist. Solche Versuche würde Hirsch wohl unter das Etikett hyper-theoreticalness einordnen. Dahinter soll allerdings ein Fragezeichen gesetzt werden. Kann nicht auch die Entscheidung für den common sense ,deep“ oder „,non-shallow“ sein? Kann man nicht auch für die Priorität der Alltagssprache und ,ihres“ Existenzquantors argumentieren, folglich dafür, dass alternative Sprachformen und entsprechende Bedeutungsvariationen in gewisser Weise sekundär, ihre semantische Bewertung immer unter dem Vorbehalt des ,als ob“ stehen? Gerade wenn man, wie Hirsch, behaupten möchte, dass es der alltagssprachliche Gebrauch des Existenzquantors ist, der die außersprachliche Realität betrifft. An dieser Stelle soll dieser Gedanke 
nicht weiter verfolgt werden. Das wird in späteren Abschnitten (v.a. in 3.21 und 3.22) ausführlicher geschehen.

Die Ausführungen über Quantorenvarianz sollen vielmehr durch den Hinweis (vorläufig) beschlossen werden, dass es in der Ontologie nicht nur um Existenzfragen geht. Dies wurde auch schon im Zusammenhang der Diskussion der Easy Ontology erwähnt, um zu zeigen, dass die Deflationierung der Existenz-Thematik (selbst wenn sie erfolgreich wäre, was hier in Frage gestellt wird) keinesfalls zu einer Deflationierung der gesamten Ontologie führt. Die Themen „Modalitäten“, „Schichten der Wirklichkeit“, ,,Verhältnisse von Vorkommnissen unterschiedlicher Kategorien“ können paradigmatisch dafür angeführt werden, dass mit dem Hinweis auf Quantorenvarianz nur ein Teilbereich der Ontologie, wenn auch ein bedeutender, berührt wird.

Zusammenfassend können wir festhalten: Im Felde von metaontologischen Positionen, welche Quantorenvarianz annehmen, sind es lediglich manche extremen Varianten (z.B. jene Ryles), aus denen sich der Versuch einer vollständigen Deflationierung ontologischer Fragestellungen ableiten ließe. Eli Hirsch würde einer solchen umfassenden Deflationierung nicht beipflichten. Er wendet sich zwar gegen eine hypertheoretische Ontologie, allerdings fasst er dabei nur jenen Teilbereich ontologischer Themen ins Auge, in dem es um Existenzbehauptungen geht. Auch in diesem Teilbereich kann man nach Hirsch nur den Gehalt mancher Dispute auflösen, nicht den aller. Und selbst bei den nach Hirschs Meinung zu Deflationierenden kann man sich fragen, ob er, Hirsch, seine Ansicht nicht auf bestimmten Annahmen inhaltlicher und ontologie-theoretischer Art gründet, etwa auf dem von ihm angenommenen Pragmatismus, welche ihrerseits kritisiert werden können. Die Vermutung legt sich jedenfalls nahe, dass durch die Ontologiekritik von Vertretern der Quantorenvarianz die Geschichte der Ontologie nicht beendet wird.

\subsection{Die Nicht-Substantialität ontologischer Fragen}

In den einleitenden Bemerkungen zum Abschnitt 2.1 wurde eingeräumt, dass in dieser Erörterung des Deflationierungsprogramms sicherlich nicht alle Positionen, ja nicht einmal die unterschiedlichen Varianten der unter 2.11-13 behandelten Theorien geschildert werden können. Es geht vielmehr um Grundlinien einer Strategie zur Verteidigung der Ontologie, die anhand der 
Auseinandersetzung mit paradigmatischen VertreterInnen des Deflationierungsprogramms entwickelt werden sollen. Dies geschieht allerdings mit dem Anspruch der allgemeinen Anwendbarkeit. Ein Aspekt dieser Strategie kann mit Bezug auf Theodore Siders Ausführungen zur Nicht-Substantialität ontologischer Fragen ergänzt werden.

Sider entwickelt ein Kennzeichen jener ontologischen Dispute, welche keine argumentativ vertretbare Lösung aufweisen, somit deflationiert werden könnten. Er charakterisiert solche „nicht-substantiellen“ ontologischen Debatten so, dass die KontrahentInnen Begriffe verwenden, die aufgrund unterschiedlicher Bedeutungen unterschiedliche, aber gleichwertige (Sider: ,equally good") Lösungen des diskutierten Problems erlauben. ${ }^{51}$ Beim Verstehen von „Gleichwertigkeit“ bei Sider ist sein (starker) Realismus zu berücksichtigen, demzufolge bzgl. der gemeinten alternativen Antworten gilt: „no candidate carves at the joints [of nature or reality] better than the others". ${ }^{52}$ Der Wirklichkeit kommt keine Antwort näher als eine andere.

Wir können die Erörterung dieser Metaphorik hier beiseite lassen und vielmehr auf ein richtungsweisendes Beispiel für solche nicht-substantiellen Dispute Bezug nehmen. Es ist der Streit über Kriterien personaler Identität. In einem einschlägigen Artikel $^{53}$ sieht Sider die Grenze der Substantialität dieser Debatte dort, wo man sich aufgrund bestimmter theoretischer Vorannahmen bzgl. der Bedeutung des Begriffs „Identitätskriterium“ auf die Alternative zwischen körperlichen bzw. psychologischen Gegebenheiten beschränkt. Fasst man „Identitätskriterium“ bei Personen physisch oder physiologisch auf, wird man als Antwort bestimmte körperliche Bedingungen finden, welche notwendig bzw. hinreichend für personale Identität sind. Geht man jedoch davon aus, dass „Identitätskriterium“ psychologisch zu interpretieren ist, wird man auf gewisse psychologische oder kognitive Instanzen, etwa im Sinn von Lockes Selbstbewusstseinskriterium, verweisen, wenn man die Identität bzw. Nichtidentität von Personen erfassen will. Nach Sider sind diese Antworten aber gleichwertig, weil sie gleichermaßen der Realität entspre-

\footnotetext{
${ }^{51}$ Sider 2011, 46, im Abschnitt 4.2 „Substantivity characterized“.

${ }^{52}$ Ebd.

${ }^{53}$ Sider 2001.
} 
chen, um Lewis' joint-carving-Metapher frei wiederzugeben. Somit wird die Frage nach personaler Identität als nicht-substantielle ausgewiesen.

Der springende Punkt aber, den Sider in diesem Artikel besonders deutlich macht, ist, dass die Wahl der beiden Kandidaten für die Bedeutung von „Identitätskriterium“ auf einer Voraussetzung beruht; nämlich der, dass sich überhaupt eine (informative) Kriteriologie für personale Identität angeben lässt. Beide Bedeutungen haben nur im Kontext einer ,complex view“ der personalen Identität, der Ansicht also, dass sich personale Identität analysieren und explizieren, letztlich damit auch reduzieren lässt, ihre Berechtigung. Nimmt man hingegen eine „simple view“ an, derzufolge personale Identität ein einfaches, nicht analysierbares Faktum ist, das auch keine (informative) Kriteriologie erlaubt, fällt jene Bedeutungsdifferenzierung weg, welche nach Sider zur Desavouierung der Frage nach personaler Identität als nicht-substantieller führt.

Mein Punkt ist es hier nicht, eine Entscheidung zwischen der simple- und der complex view bzgl. personaler Identität zu begründen. Mein Punkt ist vielmehr, dass sich die Substantialität bzw. Nicht-Substantialität ontologischer Fragen relativ zu bestimmten Voraussetzungen verstehen lässt. Darin sehe ich jenen wichtigen Aspekt der Ausführungen von Theodore Sider, der auch für die Einschätzung der Ontologiekritik der Quantorenvarianz relevant sein mag. Schon bei Hirsch haben wir gesehen, dass, wenn überhaupt, dieser Ansatz nur unter bestimmten, nochmals zu hinterfragenden Voraussetzungen $\mathrm{zu}$ einer Deflationierung gewisser ontologischer Fragen führt. Dies mag durch Sider gestützt und verdeutlicht werden: Wenn man beispielsweise die Frage, ob es durch die Zeit mit sich identische Entitäten gibt, zu einer Frage nach unterschiedlichen Bedeutungen des Existenzquantors deflationiert, welche bzgl. endurer unterschiedliche, jedoch gleichwertige Antworten (in Siders Sinn) erlauben, kann man das mit Sider auch so kommentieren, dass gerade diese Bedeutungsvariation des Existenzquantors schon vor dem Hintergrund ontologischer Entscheidungen geschieht. Diese Entscheidungen sind natürlich reflektier- und diskutierbar. Die Mühe ontologischer Dispute bleibt einem jedenfalls nicht erspart. Wenn man möchte, könnte man von hier aus auch nochmals Licht auf die Streit-um-Worte-Partei innerhalb des Deflationierungsprogramms werfen: Damit wir ontologische Dispute auf Streitereien 
über Worte bzw. deren Bedeutungen hin deflationieren können, müssen wir diese Bedeutungen auf bestimmte Weise festlegen. Dies geschieht nach Maßgabe theoretischer Bedingungen, die ihrerseits wiederum Gegenstand von (substantiellen) ontologischen Überlegungen sind.

Zugegeben: Es mag durchaus auch nicht-substantielle Fragen geben, die sich im Kontext ontologischer Debatten etabliert haben, zumindest über eine bestimmte Zeit hinweg. Dennoch bestätigt der abschließende Hinweis auf Sider, dass davon niemals die Ontologie als Ganze betroffen sein kann. Zu deflationieren sind dann, wenn überhaupt, Fragen, die einen Teilbereich ontologischer Theorienbildung betreffen, etwa Debatten, die auf die Bedeutung des Existenzquantors zurückzuführen sind. Aber auch diesbezüglich, wie wir gesehen haben, sind es nur manche, nicht alle Dispute; und auch bei den manchen gilt Siders Hinweis auf die notwendige Hebung von Voraussetzungen, welche die Nicht-Substantialität von Fragen bedingen mögen.

Diese Grundlinien einer Strategie zur Verteidigung der Ontologie können keinesfalls Originalität beanspruchen. Im Grunde, um auch den größeren Bogen wieder zu schließen, tauchen sie (oder verwandte Varianten) stets dann auf, wenn Deflationierer in Erscheinung treten. Diesbezüglich kann wieder auf die klassische Carnap-Quine-Debatte verwiesen werden. Auch Carnap deflationiert Fragen in einem Teilbereich der Ontologie, nämlich solche bzgl. Existenz, im Letzten freilich nur jene, die auf externe Fragen zurückzuführen sind. Quine hat gezeigt, dass auch diese Deflationierung auf bestimmten Voraussetzungen beruht, die hier nicht nochmals aufzulisten sind.

Wir wollen uns mit diesem Zwischenergebnis einem weiteren Programm zuwenden, das Bedenken gegen die Ontologie als philosophische Disziplin vorgetragen hat, nämlich dem Naturalisierungsprogramm. 


\subsection{Naturalisierung}

Wie eingangs des zweiten Kapitels bemerkt, beinhaltet das Naturalisierungsprogramm von den Angriffen der DeflationiererInnen unterscheidbare Attacken gegen die Ontologie. Dennoch, und darauf wollen wir hier das besondere Augenmerk legen, können auch innere Zusammenhänge zwischen diesen Ansätzen der Ontologiekritik gefunden werden. Von der Sache her ist das wenig überraschend: Wenn NaturalistInnen, pointiert auf den Punkt gebracht, behaupten, dass die wirklich relevanten Existenzfragen in den Naturwissenschaften entschieden würden, liefe dies auf eine Reduzierung ontologischer Fragen, wie es Existenzfragen sind, auf naturwissenschaftliche hinaus, somit letztlich auf eine Deflationierung der Ontologie, in eingeführtem Sinne. Somit ist es auch nicht ungewöhnlich, dass es führende AutorInnen gibt, die, hier wie da, als DeflationiererInnen und als NaturalisiererInnen, in Erscheinung treten.

Entsprechend der Zielsetzung dieses Kapitels soll es im Folgenden darum gehen, Aspekte naturalistischer Ontologiekritik in ihrem inneren Zusammenhang etwas genauer aufzulisten und darzustellen, um sie im Hinblick auf die weitere Entwicklung eines Ontologiebegriffes brauchbar zu machen. Dazu sollen deren theoretische Voraussetzungen und ihre Reichweite analysiert werden. Ziel ist an dieser Stelle freilich keine allgemeine Naturalismuskritik. Diese wird im Abschnitt 3.223 erfolgen. Unser Betätigungsfeld bleibt hier allein die Metaontologie, wie sie in der aktuellen Literatur unter Bezugnahme auf die Gründungsautoren der analytischen Ontologie betrieben wird.

\subsection{Die Metaphysikkritik der frühen Analytischen Philosophie}

Die wohl radikalste Metaphysik- und somit auch Ontologiekritik, die mit der aktuellen Metaontologie unter dem Stichwort „Naturalisierung“ in Zusammenhang gebracht werden kann, finden wir in der Frühzeit der Analytischen Philosophie; und zwar im Kontext jener Positionen, die einen radikalen „linguistic turn" in der gesamten Philosophie einfordern.

Wenn Ludwig Wittgenstein in seinem Tractatus logico-philosophicus (TLP) darangeht, ,dem Denken eine Grenze [zu] ziehen, oder vielmehr - 
nicht dem Denken, sondern dem Ausdruck der Gedanken“"54, so braucht es keine Spezialkenntnisse in Wittgenstein-Exegese um festzustellen, dass sich jene Fragestellungen, mit denen sich die Ontologie in Vergangenheit und Gegenwart beschäftigt, wohl außerhalb dessen befinden, was sich ihm zufolge sinnvollerweise sprachlich ausdrücken lässt. Natürlich ist Wittgenstein kein Naturalist. Er wendet sich sogar ausdrücklich gegen das Hauptdogma des philosophischen Naturalismus, wenn er bekundet: „Die Philosophie ist keine der Naturwissenschaften“ (TLP 4.111). Desgleichen setzt er sich ab von jener „modernen Weltanschauung“ des Szientismus, welche auf der „Täuschung“ beruht, dass die „Naturgesetze die Erklärungen der Naturerscheinungen“" seien (TLP 6.371), was ja noch eine milde und offene Formulierung dessen ist, was NaturalistInnen im Kern ihres Programms annehmen. Dennoch hat er, nolens volens, entscheidende Impulse für eine naturalistische Ontologiekritik geliefert. Etwa durch seine Festlegung darauf, dass es eigentlich die einzig richtige Methode der Philosophie wäre, „Nichts zu sagen als was sich sagen lässt, also Sätze der Naturwissenschaft" (TLP 6.53). Daraus ergibt sich, dass eine philosophische Disziplin, welche unabhängig von, oder vorab zu naturwissenschaftlichen Kontexten Existenzfragen zu klären versucht, schlicht Unsinn produziert. Das ist im Kern die Ontologiekritik des Naturalismus, auch wenn sie sich nicht (mehr) auf den Tractatus beruft.

Wittgenstein ist auch deshalb in diesem Zusammenhang zu erwähnen, weil er die Metaphysik- und somit Ontologiekritik des Wiener Kreises durchaus inspiriert hat. (Nota bene: Es geht hier nicht um die diffizile Frage nach den historischen Bezügen zwischen Wittgenstein und Vertretern des Wiener Kreises, sondern um die faktische Nähe mancher Anschauungen unter relevanter Rücksicht.) Allen voran sind die frühen programmatischen Schriften Rudolf Carnaps zu erwähnen, etwa seine Artikel zur „Überwindung der Metaphysik durch logische Analyse der Sprache“ bzw. der „Physikalischen Sprache als Basissprache der Wissenschaft“". 55

Vor dem Hintergrund einer szientistischen Weltanschauung, die Carnap u.a. in seiner wissenschaftlichen Autobiographie eindrucksvoll darlegt, ${ }^{56}$ ist

\footnotetext{
${ }^{54}$ Siehe Wittgensteins Vorwort zum Tractatus; verwendete Ausgabe: Wittgenstein 1963.

${ }^{55}$ Hier: Carnap 1931 bzw. 1931 b.

${ }^{56}$ Siehe Carnap 1993, u.a. 11.
} 
klar, dass für ihn die Naturwissenschaften universalen Geltungsanspruch haben, damit auch die Basis jedweder kognitiv relevanten, sprich ,wissenschaftlichen“ Welterklärung bilden. ${ }^{57}$ Der Philosophie bleibt die Aufgabe der logischen Klärung naturwissenschaftlicher Aussagen. ${ }^{58}$ Metaphysik und Ontologie, die sich „Objektfragen“ zuwenden, verlieren sich in sinnlosen Worthülsen. Diese Sinnlosigkeit lässt sich darauf zurückführen, dass in der Ontologie zum einen Ausdrücke syntaxwidrig zusammengestellt werden. ${ }^{59}$ Wenn etwa behauptet wird, ,Seiendes ist“", scheint hier schlicht und einfach eine unvollständige Prädikation vorzuliegen. Es fehlt das Prädikat, bzw. wird die Kopula ungerechtfertigter Weise als solches verstanden. Sinnlose Sätze kommen aber, zum anderen, auch so zustande, dass Behauptungen aufgestellt werden, welche keiner gesicherten Methode der Verifikation, das sind entweder Logik oder Empirie, zugänglich sind. ${ }^{60}$ „Substanzen sind unabhängige Entitäten“, z.B., ist weder ein logisches oder analytisches Urteil, noch empirisch bestätig- bzw. widerlegbar.

Zeitgenössische NaturalistInnen setzen sich vom einfachen, mitunter polemischen Stil der angesprochenen programmatischen Schriften ab. Der aktuelle Naturalismus ist in seiner Ontologiekritik zweifelsfrei technisch versierter und auch inhaltlich reicher. Dennoch sei es erlaubt, die Frage zu stellen, ob sich aktuelle NaturalistInnen nicht nur in ihren weltanschaulichen Rahmenbedingungen, sondern auch in den Prämissen ihrer philosophischen Theorienbildung, meist implizit, an jenem Programm orientieren, das Carnap erfrischend explizit dargelegt hat. Allen voran ist die universale Erklärungskompetenz der Naturwissenschaften zu erwähnen. Die Naturwissenschaften geben vor, was der Fall ist, die Ontologie versuche, dies in der ihr eigenen allgemeinen Begrifflichkeit nachzuzeichnen. Jedenfalls habe sich die Ontologie als ,a posteriorische“ Disziplin zu verstehen. Entspricht das nicht exakt Carnap bzw. der alleinigen Abbildungskompetenz der Welt durch naturwissenschaftliche Aussagen Wittgensteins? Wir werden diesem Punkt noch konkreter nachgehen. Ebenso dem, ob sich das von Carnap der Philosophie zuge-

${ }^{57}$ Besonders prägnant: Carnap 1931b, 448, wo Carnap davon spricht, dass die physikalische Sprache die Sprache der Wissenschaft ist.

${ }^{58}$ Siehe u.a. Carnap 1931, 237f.

${ }^{59}$ Vgl. u.a. Carnap 1931, 229.

${ }^{60}$ Vgl. Carnap 1931, $236 f$. 
wiesene Refugium, nämlich die logische Klärung naturwissenschaftlicher Aussagen, nicht auch bei aktuellen naturalistischen Ontologiekritiken findet. Wenn etwa behauptet wird, dass Ontologie, die sich nicht auf Begriffsanalyse beschränke, in unbescheidene, aber leere Spekulation verfalle.

Abschließen möchte ich diesen Abschnitt aber nicht mit der provokant klingenden Frage, ob gewisse Rahmenbedingungen von Carnaps Ontologiekritik auch heute noch Geltung haben, und es nicht wert wäre, dies in ausgefeilteren naturalistischen Ansätzen explizit zu machen und kritisch zu reflektieren. An dieser Stelle sei vielmehr nachgefragt, ob Carnaps Kritik bzw. jene der frühen Analytischen Philosophie tatsächlich jede Art ontologischer Theorienbildung trifft. Wittgenstein und Carnap hatten eine bestimmte Gestalt von Metaphysik und Ontologie vor Augen, etwa jene, die paradigmatisch in Heideggers Schrift „Was ist Metaphysik?“ exemplifiziert ist. Von dieser kann man sich tatsächlich fragen, ob sie Grammatik und Syntax standardmäßig befolgt. Ebenso sind die Begründungsstrategien Heideggers berechtigterweise $\mathrm{zu}$ diskutieren. Ohne mit erhobenem Finger auf historische $\mathrm{Au}-$ toren zu verweisen, lässt sich aber, durchaus unter Verweis auf Wittgenstein und Carnap, fragen, ob nicht auch zeitgenössische Ontologien, selbst solche analytischer Provenienz, jene begrifflichen und argumentativen Standards verfehlen, welche die beiden vor Augen hatten. Natürlich können wir aber auch festhalten, dass es Ontologie-Projekte gibt, die sich eben darum bemühen, und das durchaus unter Berufung (wenn schon nicht dem Buchstaben, so doch der Intention nach) auf die Gründungsväter der Analytischen Philosophie.

Wir halten fest: Die Ontologiekritik der frühen Analytischen Philosophie beruht auf schweren Voraussetzungen, u.a. jener, dass die Physik als „Basiswissenschaft", auch im Hinblick auf Existenzfragen, zu verstehen sei. Insofern diese Kritik auf die Ontologie als Ganze abzielt, kann ihr durch eine Infragestellung dieser Voraussetzungen entgegengetreten werden. Fasst man diese Kritik bescheidener auf, und zwar als Ausformulierung bestimmter begrifflicher und argumentativer Standards, gibt es wohl ontologische Theorien, die nicht zu Unrecht ihrem Verdikt ausgesetzt sind. Das sind, so ein optimistischer Abschluss, allerdings nicht alle. 


\subsection{Die Sinnlosigkeit aller „unbescheidenen Ontologie“}

Radikale Attacken wie jene Wittgensteins und Carnaps gegen die Ontologie, verbunden mit ihrem Programm des linguistic turn, haben sich im Zuge der Etablierung der Analytischen Philosophie bald gelegt. Es traten, vom Standpunkt der Ontologie aus gesehen, differenzierendere und moderatere Kritiken auf; auch, und das ist hier unser Thema, im Kontext des Naturalisierungsprogramms. In diesem Sinn sollen im Folgenden Ansätze behandelt werden, welche die Ontologie nicht in Bausch und Bogen als ,unwissenschaftlich“ oder ,,sinnlos“ brandmarken, sondern nur, insofern sie sich vollständig losgelöst von begrifflicher Analyse bzw. außerhalb des Kontexts naturwissenschaftlicher Theorienbildung bewegt. Unter Heranziehung eines in der aktuellen Debatte verwendeten Attributs könnte man solche Ontologie auch „unbescheiden" bezeichnen. Der wesentliche Fortschritt zur Fundamentalkritik Carnaps und Wittgensteins besteht darin, dass damit eine gewisse Vereinbarkeit von Naturalismus und Ontologie nicht ausgeschlossen wird.

Ein erster Autor, der unter diesem Etikett behandelt werden kann, ist Quine. Er ist uns schon in der Einleitung als Kritiker Carnaps begegnet. Somit können wir uns an dieser Stelle bei der Darstellung seiner Absetzung von seinem „Lehrer und Freund“ und der damit verbundenen Weiterentwicklung naturalistischer Ontologiekritik kürzer fassen. Quine kann jedenfalls so verstanden werden, dass er jene Voraussetzungen in Frage stellt, die Carnap in seinen frühen programmatischen Artikeln zur vollständigen „Überwindung der Metaphysik [...]“ präsupponiert. Es sind nicht zufällig auch jene Punkte, die ihn gegenüber Carnaps späterer Deflationierung der Ontologie zurückhaltend sein lassen. Allen voran sind das die Unterscheidung zwischen analytischen oder logischen und synthetischen oder empirischen Urteilen, sowie die damit zusammenhängende Annahme, dass man überhaupt einzelne Sätze verifizieren könnte. ${ }^{61}$ Wir haben ja gesehen, dass Carnap diese Distinktionen auch bei seiner Argumentation für die Sinnlosigkeit der Ontologie voraussetzt: Nach Carnap sind (einzelne) ontologische Sätze empirisch nicht zu verifizieren, obwohl sie beanspruchen, nicht analytisch zu sein. An dieser Stelle müssen wir uns nicht um die Detailargumente Quines kümmern, auch nicht

${ }^{61}$ Das kritisiert Quine bekanntermaßen als „Dogmen des Empirismus“, siehe Quine 1951. 
um die Frage, ob Carnap durch Quine nun tatsächlich widerlegt sei, oder vielleicht doch nicht. Auch hier setzen wir vielmehr bei Quines holistischem Theorienverständnis an, demzufolge niemals einzelne Sätze verifiziert werden können, sondern stets Theorien als Ganze, und damit auch die Unterscheidung zwischen logischen und empirischen Sätzen zu relativieren ist.

Den für diesen Kontext relevanten Aspekt können wir in den Blick bekommen, wenn wir uns fragen, welche ganzen Theorien das nun sind, die Quine in besonderem Maße interessieren. Es besteht kein Zweifel daran, dass dies für ihn naturwissenschaftliche Theorien sind, allen voran solche der Physik. Und zwar deshalb, weil es allein die Physik bzw. physikalische Begriffsschemata sind, die uns mit unserer Erfahrungswelt in adäquater Weise zurechtkommen lassen. ${ }^{62}$ Dementsprechend sieht Quine die Aufgabe der Ontologie darin, jene ontologischen Verpflichtungen in den Blick zu bekommen, die man mit diesen „besten“ wissenschaftlichen Theorien eingeht. Dazu hat man deren Aussagen in die ,kanonische Notation“ der Prädikatenlogik zu übertragen. Ontologisch verpflichtend ist der Gebrauch von Variablen (,etwas“, „x“), welche durch den sogenannten Existenzquantor („Es gibt mindestens ein“, ,ヨ“), gebunden sind. Dass etwas existiert, bedeutet somit nichts anderes als im Wertebereich gebundener Variablen vorzukommen. Im Wertebereich gebundener Variablen von Aussagen physikalischer Theorien aber kommen primär physikalische Gegenstände (engl.: physical objects) vor, worin wir den charakteristischen Ausdruck einer physikalistischen oder naturalistischen Ontologie erblicken können.

Entscheidend für das Verstehen von physikalischen Gegenständen als den Bewohnern jenes Wertebereichs, auf den man sich mit Aussagen in physikalischen Theorien verpflichtet, ist es nun aber, dass es sich bei den von Quine so angenommenen „Entitäten“ genau genommen um Setzungen (engl.: posits)

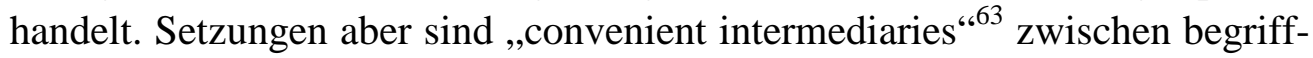
lichen Schemata und den Erfahrungen. Solche Setzungen entsprechen Modellen, mit denen wir empirische Daten und deren Zusammenhänge interpretieren, bei wissenschaftlichem Anspruch auf möglichst ökonomische, einfache

\footnotetext{
${ }^{62}$ In Quine 1951, 42, wird diese Adäquatheit auch als Vereinfachung interpretiert: Physikalische Theorien ,serve [...] to simplify our treatment of experience“.

${ }^{63}$ Quine 1951, 41.
} 
und konsistente Weise. Beispielhaft ist in diesem Zusammenhang die Annahme von Molekülen. ${ }^{64}$ Moleküle sind nicht etwas, das wir durch einen Blick in ein Elekronenmikroskop entdecken. Wir nehmen Moleküle als Setzungen an, weil uns das erlaubt, nach bestimmten physikalischen, wohl auch chemischen Begriffsbildungen, gewisse empirische Befunde in adäquater Weise modellhaft zu interpretieren.

Was wäre nun nach Quine eine „unbescheidene“ und folglich sinnlose Ontologie? Ein erstes Unbescheidenheitskriterium ist für Quine Dogmatismus. In Theorien geht es für Quine stets darum, nach pragmatischen Gesichtspunkten Interpretationen von Erfahrungen zu geben. Faktisch erfüllen physikalische Theorien mit ihren Setzungen den Maßstab solcher Interpretationen (ihm zufolge) am besten. Deshalb ist auch sein Physikalismus niemals als „Dogma“, sondern stets unter dem Vorzeichen des Pragmatismus zu verstehen. ${ }^{65}$ Dennoch, Quine ist faktisch Physikalist oder Naturalist. Deshalb ist für ihn, zweites Kriterium, wohl auch jene Ontologie unbescheiden, die nicht im Kontext der besten wissenschaftlichen Theorien arbeitet, das sind für ihn, bis zum Auftreten von Alternativen, jene der Physik. Unbescheiden wäre schließlich, drittens, auch eine solche Ontologie, die unabhängig von ontologischen Verpflichtungen, die wir mit quantifizierten Aussagen eingehen, ihre Theorien entwickelt. Dieses Kriterium könnte man als Abwendung von brachialrealistischen Verstehensweisen der Ontologie auffassen bzw. als Erbe eines moderateren linguistic turn.

Wir sehen also, dass auch Quines Ontologiekritik nicht unsere Disziplin als Ganze betrifft, sondern nur bestimmte dogmatische Varianten, bzw. solche, welche nach den Grundstrukturen der Wirklichkeit fragen, ohne Rückbezug auf unsere sprachliche Praxis. Auch können wir festhalten, dass Quines Kritik klare Voraussetzungen hat: der faktisch angenommene, für sämtliche Wissenschaften grundlegende Charakter der Physik, Pragmatismus, eine spezifische Interpretation des Existenzquantors und die Interpretation von Entitäten als Setzungen.

${ }^{64}$ In Quine 1960, §6, werden Moleküle als paradigmatische Setzungen angeführt.

${ }^{65}$ Vgl. Quine 1951, 43, wo Quine seinen Pragmatismus als über jenen Carnaps hinausgehenden erachtet. Das gilt im Speziellen für die Akzeptanz bestimmter Setzungen, aber auch im Allgemeinen für die Annahme physikalischer Gegenstände, vgl. dazu auch Quine 1960, 4. 
Nach dieser mittlerweile als klassisch zu bezeichnenden naturalistischen Version des Unbescheidenheitsvorwurfs an (manche) Ontologiekonzepte soll in diesem Zusammenhang noch eine weitere behandelt werden, die gerade in der aktuellen Metaontologie einige Beachtung findet. Es ist die von Thomas Hofweber propagierte „,bescheidene Ontologie“".66

Man kann Hofwebers Beiträge durchaus als eine Weiterentwicklung von Quines grundlegenden Ansichten verstehen, vor allem insofern Hofweber bei einer differenzierenden Analyse des Existenzquantors ansetzt. Dass er diese Weiterentwicklung unter Vorzeichen betreibt, die man in einem weiteren Sinne als „,carnapianisch“ bezeichnen könnte, sollte aus der folgenden Darstellung ebenfalls ersichtlich werden.

Einer der leitenden Gesichtspunkte bei Hofwebers Weiterführung des quineschen Ansatzes ist die Frage nach einem genaueren Verstehen von ,ontologischer Verpflichtung“, die wir durch die Verwendung des sogenannten Existenzquantors eingehen. Dem entspricht die Frage, was genau es bedeutet, wenn wir Aussagen der Form „Es gibt mindestens ein x, für das gilt, dass es F ist" behaupten. Eine Ebene der Diskussion ist, ob es denn nicht Verwendungen des besagten „Es-gibt-mindestens-eins-Quantors“ geben könnte, die gerade nicht darauf verpflichten, all das als existierend anzunehmen, worüber wir quantifizieren. Nehmen wir z.B. an, Hans sei ein Verehrer der Harry Potter-Serie. Nach der Lektüre sämtlicher Teile wird Hans zu einem aufrichtigen Bewunderer von Severus Snape. Aus der Feststellung, dass dem so ist, dass Hans eben Snape bewundert, können wir nun ableiten, dass es jemanden gibt, den Hans bewundert. Zur Verwendung unseres Quantors ist es nur noch ein kleiner Schritt: Es gibt mindestens ein x, von dem gilt, dass es von Hans bewundert wird. Trotz der offensichtlichen Gültigkeit der vorgenommenen Ableitungen, und das ist der springende Punkt, werden wir uns damit kaum auf die Existenz eines Bewunderungsobjekts, schon gar nicht von Snape, ontologisch verpflichten wollen. ${ }^{67}$ Thomas Hofweber nennt eine solche, eben nicht

${ }^{66}$ Grundlegend für diesen Ansatz sind zunächst Überlegungen in Hofwebers Artikel „A Puzzle about Ontology“. Hier: Hofweber 2005.

${ }^{67}$ Dieses Beispiel habe ich aus einer Passage (42.5 Was genau bedeutet „„“? ) meines Artikels „Sein als Existenz“, hier: Kanzian 2020, entnommen; einem Textteil, an dem ich mich in diesen Absätzen auch sonst stark orientiere. 
ontologisch verpflichtende Verwendungsweise des Quantors auch „,intern“ “68 Diese interne Verwendung kann auch im Sinne einer „substitutionellen“ Verwendung des Quantors verstanden werden, insofern wir durch Einsetzung oder Substitution eines Terms (,Snape“) für die durch den Quantor gebundene Variable (Es gibt etwas, das/der ...) einen wahren Satz erhalten. Technisch bemerkenswert ist, dass es sich bei der ins Auge gefassten Ableitung um eine Inferenz handelt, in der aus einem Satz mit einem offensichtlich referierenden Ausdruck (,Hans bewundert Snape“), auf einen Satz geschlossen wird ohne einen solchen („Es gibt jemanden, den Hans bewundert“). In diesem Sinn bezeichnet Hofweber diese ontologisch nicht verpflichtende, interne Interpretation des Quantors auch als ,inferential role reading“ desselben. ${ }^{69}$

Von dieser angenommenen internen ist jedenfalls die externe Verwendung zu unterscheiden. Sie legt bestimmte Wertebereiche fest, auf die wir uns dann auch tatsächlich ontologisch verpflichten. Diese Interpretation des Quantors nennt Hofweber dementsprechend auch die ,domain conditions reading “" ${ }^{70}$ Aufgabe der Ontologie ist es zu klären, welche Verwendungsweise des Quantors im jeweiligen Fall vorliegt. Hierin geht Hofweber über Carnap hinaus, insofern er darin tatsächlich ein der Ontologie vorbehaltenes „,sinnvolles Unternehmen“ (engl.: „meaningful enterprise“"71) sieht.

Eine durchaus vergleichbare Differenzierung hat David Chalmers vorgenommen, wenn er zwischen „leicht-“ und „schwergewichtigen“ Quantoren (engl.: lightweight/heavyweight quantifiers) unterscheidet. $^{72}$ Die ersteren kommen in unserer Alltagssprache durchgängig vor. Wenn wir zum Ausdruck bringen wollen, dass es im Haus Maier, zwischen Hans und Luise, familiär kriselt, sagen wir mitunter: „Es gibt dicke Luft zwischen Hans und Luise“. Es fällt nicht schwer, dies in quantifizierter Schreibweise darzulegen.

${ }^{68}$ Hofweber 2005, 273. Die Theorie interner Quantorenverwendung bzw. einer inferential role reading hat Hofweber in seinem Buch „Ontology and the Ambitions of Metaphysics“, hier: Hofweber 2016b, bestätigt, siehe v.a. chapter 3 und 4.

${ }^{69}$ Hofweber 2005, 273.

${ }^{70}$ Ebd., 271.

${ }^{71}$ Ebd., 282, auch: 278ff. In Hofweber 2016 wird der über Carnap hinausgehende Aspekt der „bescheidenen Ontologie“ betont, siehe u.a. ebd., 25. Hier setzt sich Hofweber von Carnap auch insofern ab, als er den externen Fragen durchaus kognitiven Gehalt zubilligt: „Both questions [internal and external] have the same status."

${ }^{72}$ Chalmers 2009. 
Chalmers meint nun, dass solche Aussagen trivialerweise korrekt sind, wenn man die verwendeten Termini entsprechend den eingeführten Usancen verwendet. Damit beanspruchen wir freilich nicht, etwas über die Grundelemente der Wirklichkeit auszusagen. Der Vergleich zu Hofwebers interner Quantorenverwendung liegt auf der Hand. Auch „Es gibt dicke Luft zwischen Hans und Luise“ kann man als Ableitungsprodukt aus Aussagen (mit durchaus referierenden Ausdrücken) verstehen, welche familiäre Zwistigkeiten besagen. Vom leichtgewichtigen Quantor ist jedenfalls der schwere zu unterscheiden: Wenn etwa ein Ontologe oder eine Ontologin mit realistischen Intuitionen bezüglich abstrakter Entitäten, in Einvernehmen mit platonisierenden GrundlagenforscherInnen in der Mathematik, mit kämpferischer Überzeugung behauptet: „Es gibt Zahlen.“ Schwere Quantifikationen sind ausdrücklich als Behauptungen in einem ontologischen Diskurs gekennzeichnet und haben nicht-triviale Korrektheitsbedingungen. Darin bestehen wohl auch Übereinstimmungen mit Hofwebers domain conditions reading des Quantors. Hier können wir es außer Acht lassen, dass Chalmers beide Verwendungsweisen des Quantors kritisiert, was ihn letztlich zu anti-realistischen Auffassungen in der Ontologie, zumindest im Hinblick auf die Interpretation von „Es gibt mindestens ein“-Aussagen, bewegt. Das schließt nicht aus, dass es so etwas wie eine fundamentale Ebene der Wirklichkeit gibt, ${ }^{73}$ die dann aber, so kann man Chalmers hier wohl verstehen, anders als mit dem standardmäBigen Apparat der Prädikatenlogik zu erreichen ist. Wie gesagt, das können wir beiseite lassen. Worum es geht, ist der Hinweis auf eine weitere Differenzierungsmöglichkeit bezüglich $\exists$, welche uns auch die Ontologie als ,bescheidene" Disziplin näherbringen soll.

Damit ist das Stichwort gegeben, um wieder zu Thomas Hofweber zurückzukommen. So stellt sich die Frage, wo genau gemäß seinem Ansatz Kritik an der Ontologie ansetzen könnte. Wohl dort, wo sich unsere Disziplin anmaßt, unabhängig von einer vorgängigen Analyse des verwendeten Quantors, irgendwelche Behauptungen über die Grundstrukturen der Wirklichkeit zu machen. Damit setzte man sich der Gefahr aus, die interne oder inferential role-Bedeutung des Quantors mit jener externen zu verwechseln, die tatsächlich domain conditioning wäre. Das könnte zur Folge haben, in der Ontologie ge-

${ }^{73}$ Vgl. ebd., 117-123. 
wisse alltägliche Redeweisen naiv umzulegen auf eine vermeintliche Theorie über Grundelemente der Wirklichkeit. In diesem Sinne existierten, um beim gegebenen Beispiel zu bleiben, nicht nur Hans und Luise, sondern genauso wie die beiden auch die dicke Luft zwischen ihnen, und womöglich auch Snape, der vielleicht den familiären Zwist ausgelöst hat. Was im gegebenen Beispiel harmlos erscheinen mag, hat in anderen Anwendungsfällen verheerende Wirkungen, sprich eine schier unüberschaubare, v.a. systematisch nicht $\mathrm{zu}$ erfassende Inflation von Entitäten und deren Kategorien. Die Ontologie könnte wohl kaum als Grundlagendisziplin anderer Wissenschaften fungieren, wenn sie unkritisch sämtliche „Es gibt mindestens ein“-Aussagen als Existenzbehauptungen aufgreifen würde. Diese Stoßrichtung der Ontologiekritik könnte durchaus auch unter Verweis auf Chalmers dargelegt werden. Wenn wir unberücksichtigt lassen, dass ein leichtgewichtiger Quantor andere Adäquatheitsbedingungen aufweist als ein schwergewichtiger, wird die Ontologie als Rahmentheorie von Existenzbehauptungen in Alltag und Wissenschaft nicht zu etablieren sein.

Die Missachtung der Unterscheidung zwischen auf interne und externe Quantorenverwendung bezogene Existenzfragen führt dazu, dass sich die Ontologie in Scheinprobleme verzettelt. Das Universalienproblem ist für Hofweber ein Beispiel. Es verschwindet, wenn man einsieht, dass es sich bei Fragen nach der Existenz von Eigenschaften eigentlich um ,interne“ handelt. $^{74}$

Inwiefern aber, um nun auch den in diesem Abschnitt maßgeblichen Gesichtspunkt anzuvisieren, können Hofwebers Thesen unter dem Vorzeichen des Naturalismus verstanden werden? Um dies besser in den Blick zu bekommen, kann es helfen, einige Passagen aus seinem Beitrag „Ambitious,

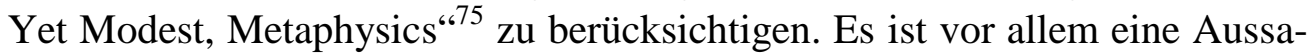
ge, die das hier Gemeinte auf den Punkt bringt: „If anyone finds entities, it's the sciences $[\ldots]^{676}$. Wörtlich genommen bedeutet das, dass die Wissenschaften, in erster Linie wohl die Naturwissenschaften, faktisch jene Instanz

\footnotetext{
${ }^{74}$ Dies hat Hofweber ausführlich in ders. 2016b, chapter 8, begründet.

${ }^{75}$ Hier: Hofweber 2009.

${ }^{76}$ Hofweber 2009, 287. An dieser Stelle fügt Hofweber zwar als Parenthese ,(for overlap cases)“ hinzu. Da dies unter der hier relevanten Rücksicht keine Einschränkung der zitierten Kernaussage besagt - mangels Relevanz der non-overlap-cases, siehe ebd., ganz oben -, kann eine Erörterung der Bedeutung des Einschubs aber unterlassen bleiben.
} 
sind, welche über Existierendes Aufschluss gibt. Das erinnert an Quine, unter der Rücksicht, dass dieser ja auch den „besten wissenschaftlichen Theorien“ bzw. deren ontologischen Verpflichtungen exklusive Kompetenz in der Klärung von Existenzfragen zubilligt. Wie aber, so können wir uns weiterfragen, ist dieser naturalistische Aspekt im Kontext der Differenzierung zwischen interner und externer Quantorenverwendung zu verstehen?

Interne Quantorenverwendung geschieht in Aussagen mit Ausdrücken, durch die wir keine ontologischen Verpflichtungen eingehen - selbst dann nicht, wenn sie aus Aussagen abgeleitet werden, in denen solche Verpflichtungen eintreten. Sprachphilosophisch gesehen gibt es in Aussagen mit interner Quantorenverwendung keine referierend gebrauchten Ausdrücke. ${ }^{77}$ Nach Thomas Hofweber sind u.a. auch Ausdrücke für Zahlen so zu verstehen. Bleiben wir zur Illustration beim Beispiel der Schafe und Ziegen, das wir schon aus dem Abschnitt über die Easy Ontology kennen. Dabei haben wir ja mit der harmlos wirkenden Aussage begonnen, dass sich gleich viele Schafe und Ziegen auf einer Weide befinden. In trivialen Schritten wurde daraus geschlossen, dass die Zahl der Schafe und die Zahl der Ziegen identisch ist, und daraus wiederum, dass es hier etwas gibt, das identisch ist, nämlich eine Zahl, nehmen wir an 26. Nach Hofweber gehen wir nun mit „26“ etwa in „26 ist die Zahl von Schafen und Ziegen auf dieser Wiese" keine Verpflichtung auf eine Entität ein, die darin bestünde, die Zahl $26 \mathrm{zu}$ sein. Mit „26“ referieren wir, so Hofweber, nicht. Unabhängig davon, was es gibt, es gibt nichts, das 26 und anderen Zahlenausdrücken entsprechen würde. Damit ist aber auch die ontologische Frage nach Zahlen beantwortet, und zwar negativ. ${ }^{78}$ Es gibt natürlich auch Existenzbehauptungen, in denen Quantoren extern gebraucht werden. Als Beispiel führt Hofweber Quantoren an, die Variablen binden, welche über einen Wertebereich mit materiellen Gegenständen (engl.: material objects) wie Dinge laufen. ${ }^{79}$ Dass dem so ist, dass es tatsächlich materiel-

\footnotetext{
${ }^{77}$ Diese These vertritt Hofweber durchgehend in 2009, bestätigt hat er sie u.a. in Hofweber 2016, siehe ebd., 27.

${ }^{78}$ Hier (Hofweber 2009, 285) bringt Hofweber ein Prinzip in Anschlag, das er auch „REF*“ nennt, dem gemäß gilt „If ,Fred“ doesn't refer to Fred then Fred doesn't exist“. Auf unser Beispiel umgelegt: Wenn wir aufgrund der Annahme des Vorliegens interner Quantorenverwendung davon ausgehen, dass ,26“ nicht referiert, existiert 26 nicht.

${ }^{79}$ Hofweber 2009, 287.
} 
le Dinge gibt, so betont Hofweber ausdrücklich, ${ }^{80}$ ist aber eine empirische Feststellung, welche von einer Naturwissenschaft, wenn schon nicht von der Physik, so doch von einer „material science“ (welcher auch immer) vorgebracht wird.

Ja, es gibt sinnvolle Fragen nach Existenz, somit eigentlich auch ein Projekt der Ontologie. Hier stellen wir nochmals einen Unterschied zu Carnap fest. Aber, zum Leidwesen für unsere Disziplin, werden diese Fragen entweder, im Fall interner Quantorenverwendung, von vornherein negativ ${ }^{81}$ oder aber, in den wirklich maßgeblichen Fällen externer Quantorenverwendung, empirisch und somit naturwissenschaftlich beantwortet. Das Herausfinden, welche Quantorenverwendung nun die adäquate ist, bleibt eine „substantielle“ und „schwerwiegende“ Angelegenheit. Allerdings, so betont Hofweber nunmehr in diesem Artikel, ergibt sich daraus ein Projekt, das die Ontologie nicht alleine bewältigen kann: „To settle internalism vs. externalism [...] is a substantial and difficult task. Here there is much work to be done, but it is largely in the philosophy of language [...]". ${ }^{82}$ Die gute Nachricht: Die Ontologie hat eine substantielle Aufgabe. Die schlechte: Eigentlich obliegt diese Aufgabe weitgehend der Sprachanalyse und Linguistik.

Inwiefern ergibt sich aus den naturalistischen Elementen der Theorie Hofwebers eine spezifische Ontologiekritik? Die Antwort liegt auf der Hand: Es sind die Naturwissenschaften, welche die verbleibenden interessanten Existenzfragen beantworten. Findet sich die Ontologie damit nicht ab und versucht unabhängig von den Naturwissenschaften externe Existenzfragen anzugehen, gleitet sie ab in eine ambitionierte, allerdings auch ungerechtfertigt unbescheidene Disziplin. Es ist wohl auch diese Unbescheidenheit, die unter Umständen sogar dazu führen kann, dass sich die Ontologie über Existenzfra-

${ }^{80}$ Ebd., Fußnote 12. In Hofweber 2016b findet sich ebenfalls der Hinweis auf die diesbezügliche Autorität der Materialwissenschaft, u.a. ebd., 197. Auch dort ist der Kontext „scientific evidence" für komplexe Makrodinge.

${ }^{81}$ „Internalism [...] answers the ontological question“, Hofweber 2009, 285. In späteren Publikationen scheint Hofweber diese These zu relativieren. Siehe ders. 2016, 29: ,If internalism is true then the external question is not answered. [...] The external question is [...] still open. Metaphysics can try to tackle it [...] ontology would be the part of metaphysics to do so." Detailfragen der Hofweber-Exegese können wir hier allerdings beiseitelassen, ohne das Hauptanliegen aus den Augen zu verlieren, das ist: naturalismus-affine Ontologiekritik.

${ }^{82}$ Hofweber 2009, 287. 
gen hinausbewegt, etwa auf Spekulationen nach der fundamentalen Ebene der Wirklichkeit bzw. der Gründung abgeleiteter Entitäten durch Bewohner dieser fundamentalen Basis; noch dazu in einer Begrifflichkeit, die außerhalb (natur-)wissenschaftlicher Intelligibilität liegt. Dies wäre in Hofwebers Diktion ,esoterische Metaphysik“. ${ }^{83}$

Damit ist auch unsere routinemäßige Frage beantwortet, welche Ontologie von Hofwebers Kritik betroffen ist. Abschließend können wir deshalb nochmals unser Augenmerk auf deren Voraussetzungen richten: Zusätzlich zur Theorie der unterschiedlichen Funktionen von Quantoren, die bereits abgehandelt wurde, und des hohen Stellenwerts linguistischer Analysen, die an die Blütezeit des linguistic turn erinnert, können wir, wie bereits festgestellt, anführen, dass Hofweber davon ausgeht, dass die Annahme der Existenz bestimmter Kategorien, wie die der materiellen Dinge, eine Sache empirischer Feststellung ist, welche den Naturwissenschaften obliegt. Wenn wir die Frage nach der Eigenart empirischer Verifikation beiseite lassen, die hier vorausgesetzt wird, können wir verweisen auf jene, den Naturalismus charakterisierende Ansicht, dass bezüglich (interessanter) Existenzfragen Naturwissenschaften prioritäre Kompetenz haben. Es ist jetzt (noch) nicht der Ort, dies zu erörtern. An dieser Stelle soll es darauf ankommen, dies als Prämisse einschlägiger Ontologiekritik festzuhalten, bzw. darauf, dass der, welcher diese Voraussetzungen nicht teilt, mit Thomas Hofweber die Ontologie nicht kritisieren kann.

\subsection{Die Nicht-Substantialität nicht-naturalistischer Ontologie}

Ein nächster Aspekt naturalistischer Ontologiekritik wird in der aktuellen Debatte durch Theodore Sider vertreten. Die Darstellung dieses Aspekts überschneidet sich mit Ausführungen im Zusammenhang mit der DeflationismusKritik (siehe oben, 2.14), wo Siders Kriterium zur Unterscheidung zwischen substantiellen und nicht-substantiellen ontologischen Fragen bereits Thema war. Dass sich aus dem Hinweis der Nicht-Substantialität (mancher) ontologischer Fragen auch unter dem Vorzeichen des Naturalismus Kritik an der Ontologie formulieren lässt, zeigt die angesprochene Überlappung ontologie-

${ }^{83}$ Ebd., 283; siehe auch ders. 2016b, chapter 13. 
kritischer Positionen (siehe oben, einleitende Bemerkungen zu 2.2). Im Folgenden wird das besagte Kriterium kurz aufgegriffen, um das Augenmerk darauf zu lenken, welche Hintergrundtheorie Sider dabei anwendet bzw. inwiefern dabei Anleihen am Naturalisierungsprogramm genommen werden.

Nicht-substantiell sind jedenfalls nach Sider jene ontologischen Dispute, welche keine argumentativ vertretbare Lösung aufweisen. Wie in Abschnitt 2.14 geschildert, charakterisiert Sider solche Dispute so, dass die beteiligten KontrahentInnen Begriffe verwenden, die, aufgrund unterschiedlicher Bedeutungen, unterschiedliche und gleichwertige Antworten auf die disputierten Fragen ergeben. Der Umkehrschluss ist natürlich auch zulässig: Substantielle ontologische Auseinandersetzungen zeichnen sich dadurch aus, dass von den KontrahentInnen Aussagen mit unterschiedlich bedeutenden Ausdrücken thesenhaft vertreten werden, welche nicht-gleichwertige Lösungen der Dispute implizieren.

Beim Versuch, besagte Gleichwertigkeit bzw. Nicht-Gleichwertigkeit zu verstehen, stoßen wir umgehend auf Siders eigenen theoretischen Rahmen. Es ist Siders Realismus, demzufolge bzgl. der alternativen Antworten im Falle der Gleichwertigkeit gilt: „,no candidate carves at the joints [of nature or reality] better than the others " 84 ; im gegenteiligen Fall aber, nicht-metaphorisch gesprochen, dass eine These der Wirklichkeit näher kommt, sie besser abbildet, als die andere. Sider geht also davon aus, dass es eine fundamentale Ebene der Wirklichkeit gibt, welche (im Fall substantieller Dispute) durch ontologische Theorien mehr oder weniger gut erfasst werden kann. Die beste ontologische Theorie ist nach Siders Ansicht eine, welche die Struktur der Wirklichkeit adäquat abbildet.

In diesem Sinn beginnt Sider auch die Einleitung seines Buches Writing the Book of the World ${ }^{85}$ mit der programmatischen Ansage eines „realism about structure" bzw. der These, dass es eine objektiv korrekte Weise gäbe, das Buch über die Welt zu schreiben. ${ }^{86}$ Wenn man versucht, diese nach Siders Ansicht beste Weise der Weltbeschreibung in den Blick zu bekommen, stoßen wir auf Merkmale, die sich vor dem Hintergrund der aktuellen meta-

\footnotetext{
${ }^{84}$ U.a. Sider 2011, 46.

${ }^{85}$ Hier: Sider 2011.

${ }^{86}$ Vgl. Sider 2011, vii.
} 
ontologischen Debatte gut rekonstruieren lassen. So ist die „wahre“ Ontologie eine, die sich jedenfalls in der Prädikatenlogik erster Stufe formulieren lässt, also jener formalen Sprache, die mit dem Existenzquantor operiert. Sider spricht in diesem Sinn (frei nach David Lewis) auch davon, dass der Existenzquantor ,,carves at the joints of nature““ ${ }^{87}$ Das, worüber wir quantifizieren, gehört also, im Unterschied zu jenen sprachlichen Zeichen, mit deren Hilfe wir quantifizierend operieren, zu jenen Elementen, welche die Grundstrukturen der Welt oder der Wirklichkeit ausmachen. In Siders Worten: „,being existential is a genuine, not merely nominal, feature of facts“ ${ }^{88}$ Darin ist ihm zufolge auch der Erfolg der Prädikatenlogik gegründet: Ihre Axiome korrespondieren strukturell mit der Wirklichkeit. Der dabei verwendete ,echte metaphysische“ Quantor ist einzig (gegen Ryle und Hirschs Pluralismus bzw. Quantorenvarianz) und in seiner „Schwergewichtigkeit“ (Chalmers) zu verteidigen. Interne Quantoren (Hofweber) lehnt Sider explizit ab. Sie sind entweder auf echte ,,joint carving“-Quantoren zurückzuführen oder zu eliminieren. Dieses Verdikt leichtgewichtiger Quantoren betrifft auch ,the quantifiers of ordinary language“. ${ }^{89}$ Die Existenzannahmen unserer Alltagssprache müssen also in die Existenzannahmen der wahren ontologischen Fachsprache (Ontologesisch, engl.: ontologese) übertragen, oder aber aufgegeben werden.

Inwiefern aber, um zum nächsten der angekündigten Schritte zu kommen, machen diese Annahmen Anleihen am Naturalismus? Sider gibt unmissverständlich zu verstehen, dass Ontologesisch letztlich die Fachsprache der Physik ist, Logik und Mengentheorie inkludierend, ${ }^{90}$ oder, mit anderen Worten, dass die fundamentale Ebene der Welt nichts anderes als die physikalische Wirklichkeit ist. Die physikalische Sprache aber ist eine, in der ausschließlich über Raum-Zeit-Punkte quantifiziert wird; Raum-Zeit-Punkte, welche mengentheoretisch zu komplexeren Gebilden zusammengefasst werden können. Spätestens im Abschlusskapitel mit dem treffenden Titel „A Worldview“91 macht Sider unmissverständlich klar: „It is a version of naturalism“. ${ }^{92} \mathrm{Im}$

\footnotetext{
${ }^{87}$ Vgl. u.a. Sider 2011, 89, aber auch 97.

${ }^{88}$ Ebd., 89.

${ }^{89}$ Ebd., $171 \mathrm{f}$.

${ }^{90}$ Ebd., 292.

${ }^{91}$ Ebd., 292-296.

92 Ebd., 293.
} 
Kontext dieses Bekenntnisses findet sich auch der für naturalistische Ontologien kennzeichnende Optimismus, demzufolge man das Reden über alltägliche Gegenstände letztlich aus der Basissprache über Mengen von Raum-ZeitPunkten rekonstruieren könne, es also eine „toy metaphysical semantics“ gäbe, selbst wenn ihm diese gegenwärtig noch nicht greifbar wäre. ${ }^{93}$

Im Unterschied zu Quine haben wir es also nicht mit einem pragmatischen Naturalismus zu tun, demzufolge die „Setzung“ (siehe oben 2.22) physikalischer Objekte als beste Interpretation empirischer Daten erfolgt, sondern einen weltanschaulich verankerten Naturalismus, der davon ausgeht, dass unabhängig von jedweder Theorienbildung die Welt fundamental besteht, eine Welt oder Natur, welche durch die Sprache der Physik allein adäquat abgebildet wird. Die wahre Ontologie, von der schon oftmals die Rede war, ist deshalb die Physik.

Welche Möglichkeiten der Kritik an der Ontologie ergeben sich daraus und auf welchen Voraussetzungen beruhen sie? Zunächst können wir auch im eben behandelten Kontext klar machen, dass nach Sider jene Ontologie zu kritisieren ist, welche sich im fruchtlosen Diskurs nicht-substantieller Fragen erschöpft. Beispiele solcher Debatten haben wir schon unter 2.14 dargelegt und müssen deshalb nicht nochmals aufgegriffen werden. Festgehalten soll hingegen werden, dass sich hier eine Überschneidung mit Kritiken aus dem Deflationierungsprogramm ergibt.

$\mathrm{Zu}$ kritisieren ist die Ontologie aus Siders Perspektive auch unter der Rücksicht metaontologischer Hintergrundannahmen, und zwar stets dann, wenn eine Abweichung geschieht von der im Grunde bereits von Quine vertretenen strikten Univozität der Bedeutung des Existenzquantors. Wenn man beachtet, dass manche Positionen, welche im Hinblick auf die Bedeutung des Quantors eine pluralistische Auffassung vertreten, dies unter dem Vorzeichen eines Versuchs der Deflationierung der Ontologie tun, kann bei Sider, neben der eben bemerkten partiellen Übereinstimmung, auch eine deutliche Absetzung von Anliegen des Deflationierungsprogramms festgestellt werden. Diese Absetzung ist auch unter Berücksichtigung von Siders eigentümlichem Realismus konsistent.

${ }^{93}$ Ebd., 294. 
Zum Stichwort „Realismus“ kann vermerkt werden, dass sich, aus Siders Perspektive, auch die Kritikwürdigkeit jeder Ontologie ergibt, welche hier in irgendeiner Weise Abstriche macht. Davon könnte Quine betroffen sein, sicherlich aber Carnap und Programme, die sich auf ihn berufen. Wenn zu existieren, wie beispielsweise nach der Easy Ontology, nichts anderes bedeutet als Applikationsbedingungen von Termini zu erfüllen, muss das nach Siders Theorie abzulehnen sein. Ihm geht es um die Welt oder die Wirklichkeit, die in ihren Strukturen zu erfassen ist, nicht um die Einführung begrifflicher Rahmenbedingungen nach pragmatischen Gesichtspunkten.

$\mathrm{Zu}$ kritisieren ist die Ontologie nach Sider schließlich auch dann, wenn sie sich in irgendeiner Weise mit dem common sense arrangiert. Nicht die Bedeutung von Existenz und Existierendem, wie es unserem alltäglichen Umgang mit uns selbst und den Dingen unserer Alltagswelt entspricht, ist wahrhaft ontologisch, sondern jene Bedeutung von „Existenz“, wie sie der physikalischen Theorienbildung, auf deren jeweils aktuellem Stand, entspricht.

Die Voraussetzungen, welche Sider macht, liegen auf der Hand: Zusammenfassend gesagt, ist das auf der Ebene der „Ideologie“994 Prädikatenlogik erster Stufe mit Quantoren univoker Bedeutung, ergänzt durch Operatoren der Mengentheorie. Auf der ontologischen Ebene ist es die Annahme vierdimensionaler Raum-Zeit-Gebilde, welche aus den primären „Entitäten“, das sind Raum-Zeit-Punkte, kompiliert werden können. Sider ist Naturalist unter den Vorzeichen eines, wie manche meinen würden, sehr starken Realismus.

Das ist nicht das Ende der Ontologie. Ein solches einzuläuten liegt sicher nicht in Siders Absicht. Es ist, wenn Sider Recht hätte, allerdings die Widerlegung jedweder ontologischer Zugangsweisen, welche seine Voraussetzungen nicht teilen. Darin erweist sich ein weiteres Mal die Relativität von Ontologiekritik, um die es in diesem Kapitel einzig und alleine geht.

\subsection{Die Unvollständigkeit nicht-naturalistischer Ontologie}

Damit kommen wir zum letzten Gesichtspunkt, der unter dem Stichwort „Naturalismus" bzw. naturalistische Ontologiekritik an dieser Stelle untersucht werden soll: Ontologie ist dann in Frage zu stellen, wenn sie nicht die gesam-

${ }^{94}$ Ebd., 292. 
te Wirklichkeit in den Blick bekommt, sondern nur manche Teilbereiche. Als exemplarischer Vertreter soll Peter Simons und sein Artikel „Farewell to Substance. A Differentiated Leavetaking“"95 behandelt werden.

Bevor wir zur Darstellung des besagten Einwands kommen, soll seine Brisanz unterstrichen werden. Die Ontologie ist Universalwissenschaft, wie es auch im programmatischen ersten Kapitel hervorgehoben wurde. Kann man einer Theorie nachweisen, dass sie Bereiche der Wirklichkeit systematisch, d.h. von ihrem Ansatz her ausklammert, kann sie keine ontologische sein. Von diesem Grundsatz geht nun Simons im angesprochenen Beitrag aus. Ein Zitat, welches das Gesagte auf den Punkt bringt: „A metaphysics [...] is a general theory of everything, or it is nothing at all."96

Es gibt wohl keinen Ontologen oder keine Ontologin, die Simons diesbezüglich widersprechen würde. Dieser Konsens mit ihm sollte allerdings dort beendet sein, wo Simons ausdrücklich macht, gegen welche Positionen er diesen allgemeinen Grundsatz meint vorbringen zu können. Auch diesbezüglich ist Simons dankenswert explizit. So schreibt er: ,a metaphysics which does not aspire to universality does not deserve the name, and it is the conceptual scheme inherent in common sense that will be incomplete for lack of coverage of outlying regions inaccessible to common sense". ${ }^{97}$ Es sind also jene ontologischen Theorien, welche sich begrifflicher Rahmenbedingungen bedienen, die vom common sense, also dem alltäglichen Verstehen der Wirklichkeit, ausgehen. Zur Begründung führt Simons an, dass solche Theorien jene Bereiche der Wirklichkeit in ihrer Reflexion ausschließen, welche eben nicht durch unser alltägliches Verstehen erfasst werden können. Das ist in erster Linie jene fundamentale Ebene der Wirklichkeit, die ihm zufolge allein von der Naturwissenschaft, vor allem der Quantenphysik, erschlossen und der Ontologie als philosophischer Disziplin zugänglich gemacht wird.

Daraus können sowohl eine grundsätzliche Einstellung zur Metaphysik oder Ontologie als auch eine konkrete These bezüglich der Ausgestaltung eines kategorialen Rahmens abgeleitet werden. Das Grundsätzliche: „Investi-

\footnotetext{
${ }^{95}$ Hier: Simons 1998.

96 Simons 1998, 251. „Metaphysics“ und „ontology“ können wir in diesem Zusammenhang übrigens cum grano salis als Synonyme gelten lassen.

${ }^{97}$ Ebd., 247, Hervorhebung Kanzian.
} 
gating the nature of the world and our relationship to it is not a task for a priori metaphysics but of a science revisable in the light of increasing knowledge about the world and ourselves, a posteriori but still with a metaphysical framework of maximal generality at any stage." 98 Nach Simons (und anderen) sind wir also als OntologInnen auf die Ergebnisse der Quantenphysik angewiesen; nicht nebensächlich, sondern im Kern der Theorienbildung, insofern es in der Ontologie (auch) um die fundamentale Ebene der Wirklichkeit zu gehen hat. Bezüglich eines kategorialen Rahmens ergibt sich daraus, dass jene für ein „,commonsensical“ Verstehen der Welt maßgeblichen Entitäten, das sind nach Simons Ansicht Substanzen, in ihrer Relevanz entscheidend relativiert werden müssen. Versteht man sie standardmäßig als dreidimensionale endurer, können sie keine Grundelemente der fundamentalen Ebene der Wirklichkeit sein. Dort kommen nämlich endurer nicht vor. Jede Ontologie, welche die fundamentale Ebene (mit)berücksichtigt, diese nicht systematisch ausblendet und so in nunmehr relevantem Sinne eben unvollständig bleibt, hat letztlich „Farewell“ zu dieser altehrwürdigen Kategorie zu sagen. Dieser Abschied kann im Zuge eines „differentiated leavetaking“ erfolgen, welcher den Stellenwert des common sense als Teilbereich der Welt, zumindest nicht von vornherein und vollständig negiert. Aber: Jede Ontologie, welche dem Vollständigkeitsanspruch gerecht werden will, muss kategoriale Schemata entwickeln, welche die Fundamentalebene der Welt in ihren Grundzügen erfasst. Sie muss darüber hinausgehen, Substanz-Ontologie zu sein.

Dass es sich bei diesem Vorschlag um einen handelt, der dem Naturalisierungsprogramm verpflichtet ist, liegt auf der Hand. In diesem Sinne plädiert Simons auch für eine ,,revisionäre Ontologie“, ${ }^{99}$ die ihre „,deskriptiv-alltäglichen“ Begriffsschemata öffnet für die Ergebnisse der Naturwissenschaft auf ihrem letzten anerkannten Stand. Die Angewiesenheit der Ontologie auf die Naturwissenschaften oder ihr ,,aposteriorischer Status“ ist dabei in einem speziellen Sinne zu verstehen, der so weit geht, dass Ontologie im Grunde zu einer Naturwissenschaft mit etwas allgemeinerer Begrifflichkeit wird. So gesehen ist Simons sogar paradigmatischer Naturalist oder Physikalist.

${ }^{98}$ Simons 1998, 251.

${ }^{99}$ Ebd., $246 f$. 
Welche Ontologien von Simons kritisiert werden, ist damit auch klar: Es sind jene sich an alltäglichen begrifflichen Schemata orientierenden ,deskriptiven" Ontologien, welche auf die Kategorie der Substanzen als grundlegende Entitäten angewiesen sind. Sie bleiben unvollständig, weil sie die fundamentale Ebene der Wirklichkeit unberücksichtigt lassen.

Es bleibt allerdings die Frage, unter welchen Voraussetzungen diese Kritik als stichhaltig angesehen werden kann. Die allgemeine Voraussetzung, dass nur eine naturalistische oder physikalistische Ontologie dem Universalitätsanspruch genügt, wurde bereits angesprochen. Ontologie muss sich also als eine aposteriorische Disziplin verstehen. Dazu sollen noch zwei besondere Prämissengruppen genannt werden: Die erste umfasst Voraussetzungen, welche auf ein Viel-Schichten-Modell der Wirklichkeit hinauslaufen. Simons selbst gebraucht ja den Begriff ,levels“, um diverse Wirklichkeitsebenen differenzierend voneinander abzuheben. Jedenfalls beruht sein Unvollständigkeitsvorwurf an Substanzontologien darauf, mit ihren kategorialen Schemata zumindest eine (von mehreren) Schichten oder Ebenen der Wirklichkeit nicht $\mathrm{zu}$ erreichen. Es ist hier nicht der Ort, dieses Viel-Schichten-Modell zu problematisieren und nachzufragen, wie sich die einzelnen Schichten oder Ebenen der Wirklichkeit nach Simons zueinander verhalten, bzw. welcher ontologische Stellenwert den nicht-fundamentalen Schichten, bis „hinauf“ zur Alltagswirklichkeit, zugebilligt wird. In diesem Kontext mag es genügen, darauf hinzuweisen, dass sich durchaus Alternativen zu diesem Modell vorstellen lassen. So könnte man die lebensweltliche Wirklichkeit des common sense als ontologisch allein maßgebliche Ebene erachten, die (Mikro-) Physik aber als Abstraktionsprodukt unter methodisch eingeschränkter Perspektive. Auch das Umgekehrte ist natürlich denkbar: Nur die (Mikro-)Physik hat ontologische Relevanz, alles andere ist letztlich Illusion. Wie gesagt: An dieser Stelle soll das Viel-Schichten-Modell nicht gegenüber Ein-Ebenen-Konzepten abgewogen, sondern als Voraussetzung des Unvollständigkeitseinwands genannt werden.

Mit diesem Modell geht bei naturalistischen Konzepten jedenfalls eine Priorisierung der ,untersten“ Schicht einher, sprich jener Ebene der Wirklichkeit, welche die (Mikro-)Physik am aktuellen Forschungsstand als grundlegend präsentiert. Von diesem Grund auf wird die Wirklichkeit „bottom-up“ 
rekonstruiert. Auch diesbezüglich soll in diesem Abschnitt keine kritische Debatte erfolgen. An dieser Stelle soll allerdings unterstrichen werden, dass es durchaus auch die alternative Möglichkeit gibt, die Wirklichkeit „topdown" zu verstehen, also der Makrowelt mit ihren Bewohnern eine Vorrangstellung einzuräumen. Bottom-up bleibt als Vorrangrichtung eine Voraussetzung bzw. steht für eine zweite Voraussetzungsgruppe, derer sich Peter Simons faktisch bedient.

Simons geht es sicher nicht darum, das Ende der Ontologie zu propagieren; „lediglich“ das Ende der Substanzontologie oder, allgemeiner gesagt, das Ende jedweder Ontologie, die sich am common sense orientiert. Die Ontologie überlebt im Schlepptau der Quantenphysik. Wäre das jemandem allerdings zu wenig für das Fortbestehen einer philosophischen Disziplin, kann er Simons Überlegungen auch als Fundamentalkritik an unserer Disziplin verstehen; gesetzt den Fall natürlich, Simons Voraussetzungen stellen sich als legitime Präliminarien heraus und erweisen seinen theoretischen Rahmen als korrekt.

Damit kommen wir an das Ende unserer Darlegung ontologiekritischer Positionen unter dem Vorzeichen des Naturalismus. Auf den Punkt gebracht stellen sie sich heraus als jeweils auf bestimmte methodische und inhaltliche Ansätze gerichtete Einwände, die relativ zu teils explizit gemachten, teils aber implizit bleibenden Voraussetzungen vorgebracht werden. Darin kommt die Ontologiekritik des Naturalismus mit jener des Deflationierungsprogramms überein. Mit diesem Ergebnis können wir zur dritten angekündigten Stoßrichtung der Ontologiekritik übergehen, welche unter dem Stichwort „Simplifizierung“ zu umreißen ist. 


\subsection{Simplifizierung}

Während die Ontologiekritik unter den Stichwörtern „Deflationierung“ bzw. „Naturalisierung“ allgemein gut eingeführte Topoi sind, ist „Simplifizierung“ keine standardmäßige Bezeichnung einer anti-ontologischen Positionierung. Deshalb soll an dieser Stelle eine kurze allgemeine Charakterisierung dessen erfolgen, was im Folgenden darunter verstanden wird. Unter „Simplifizierung" ist das Beenden von Reflexion gemeint, welches allerdings nach Maßgabe der Erfordernisse unserer Disziplin als frühzeitig und folglich unangemessen geschieht. Je umfassender ein solcher unangemessener Reflexionsabbruch in der Ontologie attestiert wird, umso grundlegender ist sie unter dieser Rücksicht zu kritisieren. Zumal, und das ist die Zielrichtung einschlägiger Kritik, der besagte Reflexionsstopp inhaltlich gesehen zur Ausblendung von Bereichen führt, die eigentlich von der Ontologie berücksichtigt werden müssten. Formal oder methodisch gesehen, führt Simplifizierung zu einer Art von Dogmatismus, der den erfolgreichen Fortschritt der Disziplin behindert.

Die Ausblendung von Bereichen, die von der Ontologie selbst berücksichtigt werden müssten, soll diese Stoßrichtung der Ontologiekritik übrigens absetzen von jener Kritik, welche abzielt auf die Ausklammerung bestimmter Fragen anderer philosophischer Disziplinen. Wenn etwa der Ontologie vorgeworfen würde, dass sie bei ihrer Analyse der Grundstrukturen der Wirklichkeit die Frage der Erkennbarkeit dieser Strukturen ausblendet, also der Epistemologie keinen primären Status einräumt. Während eine solche Kritik „von außen“ an dieser Stelle nicht behandelt werden kann, wollen wir uns der Simplifizierung widmen, insofern sie der Ontologie „von innen“ her, also nach Maßgabe dessen, was sie nach eigenem Anspruch leisten sollte, vorgeworfen wird.

\subsection{Simplifizierung und das Ausblenden naturwissenschaftlicher Forschungsergebnisse}

Ein erster Vorwurf der Simplifizierung an die Ontologie steht eng im Zusammenhang mit Einwänden aus dem Naturalisierungsprogramm, hier v.a. unter der im Abschnitt 2.24 angesprochenen Rücksicht. Er soll in diesem Kontext aufgegriffen werden, um auch von der Perspektive dieses Abschnittes aus die 
wechselseitige Verbindung verschiedener Positionen der Ontologiekritik zu verdeutlichen. Die Behandlung soll allerdings, aufgrund der besagten weitgehenden Überlappung, in gebotener Kürze erfolgen.

Auf den Punkt gebracht, wird der Ontologie vorgeworfen, in ihrer Reflexion auf die Grundstrukturen der Wirklichkeit die Ergebnisse jener wissenschaftlichen Disziplinen auszublenden, welche es gerade mit diesen Grundstrukturen zu tun haben. Das sind die Naturwissenschaften. Damit kann die Ontologie ihre eigentliche Aufgabe nicht erfüllen. Ohne weitere Rechtfertigung bleibt sie somit in ihrer Theorienbildung beschränkten Paradigmata verpflichtet, etwa jenen, die auf ein alltägliches oder vorwissenschaftliches Verstehen der Wirklichkeit abzielen. Das sei eine Art Dogmatismus. Dass diese beschränkten Paradigmata im Vergleich zu den methodisch komplexen, etwa der Naturwissenschaften, einfach, ja simpel anmuten, macht noch deutlicher, dass dieser Vorwurf auch unter dem nunmehr zur Debatte stehenden Stichwort abgehandelt werden kann.

Zur Konkretisierung sei auf einen Beitrag von Markku Keinänen verwiesen. Nach ihm ist die Ontologie dann zu kritisieren, wenn sie in ihrer Theorienbildung kategoriale Schemata entwickelt, welche auf jene Entitäten, die durch (natur-)wissenschaftliche Theorien eingeführt würden, nicht angewendet werden können (engl.: ,to be inapplicable to entities introduced by scientific theories" ${ }^{100}$ ). Diese Unanwendbarkeit ist durch einen Reflexionsstopp bedingt, der nach Maßgabe der eigenen, ontologischen Aufgabenstellung ungerechtfertigt bleibt. Auf Entitäten anwendbar zu sein, ist ja geradezu innerstes Anliegen ontologischer Theorien. Auch der Aspekt der „Dogmatisierung" lässt sich bei Keinänen finden. So wirft er Ontologien mit unanwendbaren kategorialen Schemata vor, sie seien „,based on the rather limited data about reality available to us at a given time“. ${ }^{101}$ Beschränkte Ausgangsdaten als Grundlage universaler Theorien anzunehmen, ohne diese Limitierung methodisch zu reflektieren, ist der hier vorgebrachte Kritikpunkt. Damit simplifiziert die Ontologie im eingangs eingeführten Sinn.

${ }^{100}$ Keinänen 2008, 48.

${ }^{101}$ Ebd., 46. 
In seinen Ausführungen orientiert sich Keinänen an Peter Simons, primär an seinem Artikel „Metaphysical Systematics: A Lesson from Whitehead“. ${ }^{102}$ Auch in diesem Beitrag wirft Simons einer sich am common sense orientierenden Ontologie vor, Gegenstandsbereiche, welche für ihre Reflexion eigentlich maßgeblich wären, auszublenden. Das sei unstatthaft, denn: “[...] there is much more in and to the world than common sense tells us there is". ${ }^{103}$ Es gibt, es existiert mehr als ein alltägliches Verstehen der Wirklichkeit erfassen kann. Eine Ontologie - Simons spricht auch in diesem Beitrag von „metaphysics” - kann es sich nicht erlauben, jenes „mehr“, das allein die Naturwissenschaften erfassen, systematisch zu vernachlässigen. Ohne sich an den „,besten Standards“ der Erfassung der Wirklichkeit zu orientieren, gerate die Ontologie in ein selbst verschuldetes Abseits. ${ }^{104}$ Auch von Simons her können wir den Simplifizierungsvorwurf sowohl inhaltlich als auch methodisch deutlich nachvollziehen.

Es ist nicht schwer, die Voraussetzungen dieser Version der Ontologiekritik in den Blick zu bekommen: Sie stehen in engem Zusammenhang mit denjenigen des Naturalisierungsprogramms und überlappen mit den unter 2.24 dargelegten. Allerdings können sie unter der hier maßgeblichen Rücksicht darauf fokussiert werden, dass die Naturwissenschaften in einem speziellen Sinn Entitäten einführen (Keinänen) bzw. das für die Ontologie maßgebliche Paradigma der Erklärung der Wirklichkeit bereitstellen (Simons). Lässt die Ontologie die wissenschaftlich aufgewiesenen Entitäten außer Acht, simplifiziert sie in eingeführtem Sinne.

Auch liegt es auf der Hand, gegen welche Ontologie dieser Vorwurf gerichtet ist. Es ist jene Theorienbildung gemeint, die bereits im Abschnitt über die „Unvollständigkeit nicht-naturalistischer Ontologie“ ins Visier geraten ist. Auch aus der Perspektive dieses Abschnitts können wir die Kritik nur dann als Fundamentalkritik auffassen, wenn es keine Perspektive einer naturalisierten Ontologie gäbe. Das freilich wird von den KritikerInnen nicht behauptet. Referenzobjekte ihrer Kritik sind jene ontologischen Ansätze, die

\footnotetext{
102 Hier: Simons $1998 b$.

103 Simons 1998b, 379.

${ }^{104}$ Ebd.: "A metaphysics which aspires to remain in touch with the best knowledge offered by science cannot afford to be standoffish."
} 
sich bei der Interpretation der Wirklichkeit an gewöhnlichen oder alltäglichen Begriffsschemata orientieren. Um das Kind wie im Abschnitt 2.24 beim Namen zu nennen: Sie meinen deskriptive oder Alltagsontologien.

Die Ontologie als Disziplin gegen diese Version des Simplifizierungsvorwurfs verteidigen kann man folglich entweder durch Aufbau einer ,wissenschaftlichen Ontologie“ im Sinne des Naturalisierungsprogramms oder durch eine gegenteilige Strategie, die in einer möglichst restlosen Widerlegung der eben dargelegten Voraussetzungen besteht. Hier wird der zweite Weg eingeschlagen. Ansätze zu einer nicht-resignativen Entgegnung der eben behandelten Kritik, also zur Verteidigung einer deskriptiven oder Alltagsontologie, sollen aber in einschlägigen Abschnitten des dritten Kapitels angeboten werden.

\subsection{Simplifizierung unter dem Vorzeichen naiver Hypostasie- rungen sprachlicher Schemata}

Der Simplifizierungsvorwurf kann auch unabhängig von naturalistischen Voraussetzungen erhoben werden. So beruht eine besonders weit verbreitete kritische Grundeinstellung gegenüber der Ontologie darauf, dass sie sprachliche Strukturen naiv oder eben simplifizierend als Strukturen der Wirklichkeit auffasse. Auch diese Simplifizierung sei Folge eines unangebrachten Reflexionsabbruchs, der inhaltlich zu einer ungerechtfertigten Vergegenständlichung oder Hypostasierung von Termini führt, methodisch aber zu einem allzu einfachen Verstehen des Verhältnisses sprachlicher Ausdrücke und dem durch sie Bezeichneten.

Unter dieser generellen Charakterisierung lassen sich eine Reihe von ontologiekritischen Positionen einordnen, die nochmals $\mathrm{zu}$ unterteilen sind in solche, welche konkrete sprachliche Funktionen bzw. ihre Missdeutung durch die Ontologie ins Auge fassen, bzw. solche, welche ein allgemeines Missverstehen von sprachlichen Strukturen monieren. ${ }^{105}$

\footnotetext{
${ }^{105}$ Wenn z.B. Liske in ders. 2004, 17, davor warnt, der Oberflächenstruktur der Sprache im Kontext ontologischer Überlegungen leichtfertig als Autorität zu vertrauen, meint er wohl paradigmatisch ein Missverstehen im hier anvisierten allgemeinen Sinn.
} 


\subsection{Allgemeines Missverstehen sprachlicher Strukturen}

Eine Möglichkeit, das Projekt einer Ontologie ausgehend von einem allgemeinen Missverstehen von sprachlichen Strukturen bzw. von sprachlichen im Verhältnis zu kategorialen Strukturen anzugehen, hat Keinänen im bereits angesprochenen Artikel unter dem Stichwort des „Modellierens“ (engl.: modelling) oder eines „Modellismus“ dargelegt. ${ }^{106}$ Einschlägige Modellierungsverfahren beginnen bei Beschreibungen der Welt, die ihre AutorInnen relativ zu angenommenen Rahmenbedingungen für die wahren halten. Diese Beschreibungen können auch formalisiert bzw. durch Modaloperatoren angereichert werden. Dann wird der Inhalt der Beschreibungen durch bestimmte Modelle angegeben, wobei im Falle von „starkem Modellieren“ (engl.: strong modelling) jede Distinktion auf der Ebene sprachlicher Einheiten in einer Distinktion von Modellelementen abgebildet wird. Bei „schwachem Modellieren“ (engl.: weak modelling) wird diese Strukturisomorphie nicht streng angenommen. Als paradigmatischen (weichen) Modellierer führt Keinänen übrigens David Lewis mit seinem Possibilismus an, den dieser von einer Theorie des „Inhalts“, vielleicht sollten wir eher sagen der semantischen Bewertung von Modalaussagen her entwickelt. Ohne jetzt auf Details dieser Differenzierung eingehen zu können, soll der entscheidende Punkt hervorgehoben werden: Modellierende oder modellistische Ontologien gehen einen Schritt weiter, indem sie die entworfenen Modelle als Entitäten, also als Grundelemente der Wirklichkeit, auffassen. So mache Lewis semantische Bewertungsmodelle zu Entitäten, genauerhin zu ganzen Welten von Entitäten. ${ }^{107}$ Genau genommen werden hier zwar nicht sprachliche Elemente und ihre Zusammenhänge vergegenständlicht oder hypostasiert, sondern Modelle, welche deren Inhalt erläutern sollten. Simplifiziert, im Sinne eines ungerechtfertigten Reflexionsabbruchs hinsichtlich der Legitimität eines solchen Verfahrens, wird allemal; und zwar durchaus so, dass die Simplifizierung offensichtlich auf einem bestimmten, nicht weiter hinterfragten Verständnis des Verhältnisses von sprachlichen und kategorialen Strukturen beruht.

\footnotetext{
${ }^{106}$ Dazu grundlegend: Keinänen 2008, 24ff.

${ }^{107}$ Hier kann es nicht darum gehen, Keinänens Kritik an Lewis zu beurteilen. Es geht um das, was Keinänen „Modellieren“ nennt. Für eine etwas weiter führende Darlegung von Lewis’ modalem Realismus siehe Runggaldier/Kanzian 1998, 84-90.
} 
Eine anders gelagerte, jedoch ebenfalls unter dem Stichwort „Simplifizierung“, Abteilung allgemein linguistische Simplifizierung, einzuordnende Position hat Matti Eklund unter dem Titel „Maximalismus“ dargelegt. Nach diesem Maximalismus existieren alle „quasi-möglichen“ Objekte (engl.: quasipossible objects). ${ }^{108}$ Quasi-mögliche Objekte aber sind Vorkommnisse einer bestimmten Art F, für die gilt, dass es (irgend-)eine Sprache gibt, in der die Behauptung „Fs existieren“ wahr ist. Im Hintergrund steht dabei eine möglichst liberale, sprich plurale Sichtweise von Sprachen bzw. Bedeutungen von Existenzbehauptungen in diesen Sprachen. Unter dieser Rücksicht greift Eklund jenen Pluralismus auf, den wir auch bei der Theorie der Quantorenvarianz (Hirsch) behandelt haben; allerdings ohne die bei Hirsch geschilderte Differenzierung zwischen einer eigentlichen und einer Als-ob-Quantorenbedeutung anzunehmen. Hinsichtlich Quantorenbedeutungen besteht maximale Liberalität. Somit existiert nach dem Maximalismus (trivialerweise ${ }^{109}$ ) alles, was in den wahren Sätzen irgendeiner Sprache behauptet wird. Grundstrukturen der Wirklichkeit werden in diesem Sinne simple Abbildungen sprachlicher Strukturen. Wir können hier nicht nachverfolgen, inwieweit sich Eklunds Maximalismus, wie er selbst meint, auf Quine bzw. Frege ${ }^{110}$ beziehen lässt. Festzuhalten ist allerdings, dass auch diese Position unter der Rücksicht einer Hypostasierung sprachlicher Ausdrücke verstanden werden kann. Eklund scheint dies selbst zu bestätigen, wenn er metaphorisch davon spricht, dass Objekte eigentlich „Schatten von singulären Termen seien“. ${ }^{111}$ Mit der Etablierung einer Sprache werden auch „deren“ mögliche Objekte als Entitäten, Grundelemente der Wirklichkeit, angenommen. Simplifiziert wird dabei jedenfalls, auch durch den Abbruch der Reflexion darauf, was nun tatsächlich unter einer ontologisch relevanten Sprache zu verstehen ist, bzw. welches Wahrheitskriterium angewendet wird, um nach maximalistischen Voraussetzungen quasi-mögliche Objekte einzuführen. ${ }^{112}$ Ebenso fehlt die Reflexion

\footnotetext{
${ }^{108}$ Eklund 2006, 325.

${ }^{109}$ Ebd.

${ }^{110}$ Ebd., 326f.

${ }^{111}$ Ebd., 325: ,objects are [...] mere shadows of singular terms“.

${ }^{112}$ In Eklund 2008, section IV, gibt Eklund ein empirisches Kriterium an, ,given that the empirical facts are exactly what they are", um wahre Sätze etwa über Phlogiston auszuschließen. Den Simplifizierungsverwurf entkräftet dies wohl nicht. Ganz im Gegenteil: An der an-
} 
darüber, was einen solchen rein linguistischen Zugang zur Ontologie (engl.: linguistic approach to ontology) legitimieren würde.

Wenn Eklund ein solches maximalistisches Bild einer Ontologie zeichnet, skizziert er also das Projekt einer Ontologie, welche dem Simplifizierungsvorwurf ausgesetzt ist. Da man sich in dieser Konzeption durchaus Sprachen vorstellen kann, welche außerhalb von naturwissenschaftlicher Theorienbildung definiert werden, scheint Eklunds Simplifizierung außerhalb der unter 2.31 abgehandelten Kritik zu liegen. Überlappungen bestehen jedoch mit der Ontologiekritik unter den Vorzeichen der Deflationierung. In der Tat scheint eine maximalistisch simple Ontologie auf bestimmten Voraussetzungen $\mathrm{zu}$ beruhen, etwa bzgl. Quantorenpluralismus, welche im Kontext deflationistischer Ontologiekritik (siehe 2.13) behandelt wurden. ${ }^{114}$ Auch von hier aus sehen wir jedenfalls, dass die großen Stoßrichtungen der Attacken gegen die Ontologie in einem inneren Zusammenhang stehen.

Die Darstellungen des Modellismus und des Maximalismus unterscheiden sich dadurch, dass Erstere Ontologie rein als Objekt von Kritik zeigt, Letztere aber zunächst als eine von einem Autor selbst vertretene Position. Modellismus und Maximalismus kommen jedoch in ihrer grundsätzlichen Konzeption von Ontologie überein. Diese besteht in einem linguistischen Zugang (engl.: linguistic approach) zu Fragen der Grundstrukturen der Wirklichkeit. Der Modellismus hypostasiert Modelle, die zur semantischen Bewertung sprachlicher Strukturen dienen, der Maximalismus vergegenständlicht Sprachen auf eine Weise, der gleich noch näher nachgegangen wird. Da man als Ontologin bzw. Ontologe weder Modellismus noch Maximalismus als Teil einer ontologischen Theorie vertreten kann, sondern nur als metaontologische Voraussetzung einer universalen und allgemeinen Weltbeschreibung, ist die Ontologie davon als Ganze betroffen, wenn eine der beiden Positionen zutrifft. Mit anderen Worten: Ist die Ontologie entweder modellistisch oder maximalistisch grundgelegt, kann der Simplifizierungsvorwurf gegen sie als ganze Disziplin erhoben werden.

gegebenen Stelle gibt es Eklund ausdrücklich als Ziel seiner Theorie an, die Ontologie als „,dekadent“ darzustellen.

${ }_{113}$ Eklund 2006, 327.

114 In Eklund 2008, section V, stellt der Autor den Maximalismus als eine Fortentwicklung des Deflationismus dar, relativ zum Pluralismus von Putnam und Hirsch. 
Beide Konzeptionen können freilich hinsichtlich ihrer Voraussetzungen getrennt voneinander analysiert werden, und zwar über die allgemeine Prämisse eines „linguistic approach“ hinaus. Die augenscheinlichste und auch bemerkenswerteste Voraussetzung einer modellierenden Ontologie besteht darin, dass man Modelle, wozu auch immer man sie ursprünglich entwirft, realistisch interpretieren kann, ja muss. David Lewis scheint dazu ausdrücklich zu stehen, wenn er betont, dass es eben kein „semantisches Paradies zum Nulltarif" "115 geben könne, sprich keine modellhafte Bewertung von Modalaussagen ohne Ontologie möglicher Welten. Diffiziler scheinen die Voraussetzungen von Eklunds Maximalismus zu sein. Damit nämlich singuläre Ausdrücke tatsächlich „ontologische Schatten werfen“, sprich Entitäten einführen, muss man ein spezielles Verstehen der Referenz dieser singulären Ausdrücke voraussetzen. Vereinfacht gesagt muss man annehmen, dass, wenn singuläre Termini (n) in wahren Sätzen $\mathrm{P}(\mathrm{n})$ auf Fs referieren, daraus folgt, dass es Fs gibt, dass sie existieren. ${ }^{116}$

Mit dieser Voraussetzung befinden wir uns aber auch schon im Übergang zur eingangs angekündigten speziellen Simplifizierungskritik an der Ontologie: dass sie nämlich in ihrem Verstehen bestimmter sprachlicher Funktionen, in unserem Fall des Referierens, grob vereinfachend vorgehe; wobei „grob“ bedeutet, relativ zu ihrem Anspruch simplifizierend. Sie unterlässt sowohl inhaltlich als auch methodisch die erforderliche Reflexion.

\subsection{Simplifizierendes Verstehen von Referenz}

William L. Craig hat die Ontologiekritik unter der Rücksicht eines simplifizierenden Referenzschemas neuerdings auf den Punkt gebracht. Sein Hauptanliegen in einschlägigen Publikationen ist Anti-Platonismus. ${ }^{117}$ Seine argumentative Hauptstoßrichtung besteht darin aufzuzeigen, dass sich platonisierende Ansichten, z.B. in der Philosophie der Mathematik, auf naive Referenzschemata stützen, die sich auf simple Deutungen der Verwendung von Quan-

\footnotetext{
${ }^{115}$ Vgl. Lewis 1986, „A Philosophers“ Paradise“, v.a. 4.

116 Vgl. Eklund 2006, 325.

117 Craig 2012: God and Abstract Objects; ders. 2014: Anti-Platonism; ders. 2017: Abstrakte Gegenstände, Naturalismus und Gott.
} 
toren zurückführen lassen. Sagen wir etwa aus ,,5 ist eine ungerade Zahl“, so würde aus der Verwendung von ,5“ an Subjektstelle seine Referenz auf etwas abgeleitet werden, das eine Zahl ist, also als abstrakte Entität existiert; bzw. aus: „Es gibt etwas, das die Zahl 5 und ungerade ist“, gefolgert, dass die durch „Es gibt ...“, den Existenzquantor, gebundene Variable „etwas“ über einen Wertebereich eben von abstrakten Gebilden wie Zahlen läuft.

Die von Craig verfolgte Gegenstrategie kann mit dem Stichwort „Neutralismus“ auf den Punkt gebracht werden: Selbst wenn wir wahre Sätze äußern, wie den einfachen Beispielsatz, verpflichten wir uns nicht darauf, jene Gegenstände als existierend anzunehmen, auf die sich die singulären Ausdrücke unserer Aussagen beziehen, bzw. über welche wir mit jenen gebundenen Variablen quantifizieren, welche in der prädikatenlogischen Struktur dieser wahren Sätze enthalten sind. Kurzum: Selbst wenn wir über Zahlen und Mengen sprechen, müssen wir nicht annehmen, dass Zahlen und Mengen existieren. Craigs Anliegen ist es also darzulegen, dass durch Quantoren gebundene Variablen bzw. singuläre Ausdrücke gerade keine ,ontologischen Schatten werfen“, um Eklunds Metapher aufzugreifen. Wir bleiben mit „Es gibt...“-Aussagen im ontologisch neutralen Bereich.

Die „bzw.“-Wendungen im vorhergehenden Absatz sollen darauf hinweisen, dass man bei Craig zwischen der Ebene einer Analyse der Referenz singulärer Ausdrücke und jener einer Untersuchung ontologischer Verpflichtung durch gebundene Variablen unterscheiden kann. Obwohl die Ebenen natürlich in einem inneren Zusammenhang stehen - kein Referenzschema ohne Deutung ontologischer Verpflichtung durch Quantoren - und auch die Stoßrichtung beider Stufen, wir können sie auch referenztheoretisch und metaontologisch nennen, bei Craig dieselbe ist, nämlich Anti-Platonismus, tun wir gut daran, diese Differenzierung im Auge zu behalten.

Wenden wir uns zunächst der metaontologischen Ebene des Neutralismus zu. Hier plädiert Craig dafür, Quantoren als Vehikel zu verstehen, bestimmte logische Schlussfolgerungen zu erleichtern, ${ }^{118}$ gerade aber nicht als sprach-

118 So macht die prädikatenlogische Struktur der einzelnen Aussagen transparent, dass aus „Es gibt etwas, das die Zahl 5 und ungerade ist“" folgt „Es gibt etwas, das die Zahl 5 ist“. 
liche Mittel zum Ausdruck von Existenzannahmen. ${ }^{119}$ Craig spricht sich dementsprechend für ontologisch harmlose oder neutrale Quantoren aus, die sehr an Chalmers' leichtgewichtige oder Hofwebers interne erinnern. Allerdings zieht Craig keine schwergewichtigen bzw. externen in Betracht, wie das Chalmers und Hofweber tun. Darin zeigt sich auch deutlich die Nähe von Craigs neutralistischem Vorschlag zum Deflationismus in einer doch radikalen Variante.

Craig kann mit seiner Affinität zum Deflationismus freilich nicht eindeutig in Richtung Neo-Carnapianismus interpretiert werden. So erwägt er noch ein weiteres metaontologisches Rahmenkonzept für seinen Neutralismus, den Meinongianismus. Nach einem meinongianischen Verstehen verpflichtet man sich mit Quantoren nicht auf die Existenz von Gegenständen. Das kommt dem Neutralismus entgegen. Man verpflichtet sich allerdings darauf, dass es etwas in ihrem Wertebereich gibt; darauf anzunehmen, dass uns etwas in irgendeiner Weise als Objekt gegeben ist. Von diesem kann man auch dann wahrheitsgemäß Eigenschaften aussagen, wenn man seine Existenz nicht annimmt. Das klingt attraktiv, wenn man etwa von mathematischen Gegenständen bestimmte formale Eigenschaften aussagen möchte, ohne die Existenz dieser Gegenstände annehmen zu wollen. Der Preis des Meinongianismus ist jedoch hoch. Das zeigt sich u.a. dann, wenn wir die (Nicht-)Existenz von etwas explizit behaupten wollen, wie etwa in der Aussage: „Zahlen existieren, Einhörner aber nicht.“ Wollen wir dem Sinn geben, müssen wir „Existenz“ als Prädikat im Skopus des Quantors verstehen, etwa derart, dass wir aussagen: „Es gibt etwas, das eine Zahl ist und existiert und es gibt etwas, das ein Einhorn ist und nicht existiert." Aus dem Quantor wird ein nicht auf Existenz verpflichtender „Seins-“ oder „Partikularquantor““120 ,Existenz“ aber zu einem Prädikat erster Stufe, das von einem Teilbereich dessen prädiziert wird, worauf wir mit „etwas“ Bezug nehmen. Das aber steht der gesamten Kant-Frege-Russell Tradition diametral entgegen und birgt die Gefahr in sich, letztlich zwischen dem, was es im Sinne des Partikularquantors gibt,

${ }^{119}$ Vgl. Craig 2014, 119; ders. 2017. Abschnitt: Das Unverzichtbarkeitsargument Überprüft. Um beim gegebenen Beispiel zu bleiben: Aus der logischen Durchsichtigkeit der Gültigkeit des Schlusses in der vorhergehenden Fußnote muss nicht gefolgert werden, dass etwas existiert, Grundelement der Wirklichkeit ist, das darin besteht, die Zahl 5 zu sein.

${ }^{120}$ Letztere Bezeichnung stammt von Albert Menne, in: Weingartner 1966, 57. 
und dem Existierenden $\mathrm{zu}$ unterscheiden. Dieser Aufspaltung zwischen „Sein“ und „Existenz“ begegnen aber so manche (u.a. van Inwagen ${ }^{121}$ ) mit begründeter Skepsis.

Hier soll freilich keine Widerlegung des Meinongianismus angestrebt werden. Es sei allerdings festgehalten, dass er eine keineswegs unkontroverse mögliche Konsequenz neutralistischer Deutungen der Quantoren ist. Craigimmanent könnte man sich zudem fragen, ob das Zugestehen des (meinongianischen) Seins platonischer Abstrakta, auch unter Berücksichtigung seiner eigenen theoretischen Interessen, wirklich unproblematischer ist als die Annahme ihrer Existenz. Wie auch immer: Will man sich als Neutralist mit dem Meinongianismus nicht allzu intensiv verbünden, kann man noch den anderen Weg wählen, nämlich die Akzeptanz einer Variante deflationierender Deutungen des Existenzquantors. Dass auch dies nicht ohne Einwände bleibt, wurde bereits ausführlich erörtert (siehe Abschnitt 2.1). Wir können uns deshalb gleich dem explizit referenztheoretischen Teil von Craigs Neutralismus zuwenden.

Dabei versucht Craig seine Auffassung durch eine Referenztheorie (u.a. vertreten von Båve) zu stützen, die ebenfalls durch das Attribut ,deflationistisch" charakterisiert wird. Sie ist uns übrigens bereits begegnet, nämlich bei der Einführung des Begriffs „Deflationierung“ zu Beginn des Abschnitts 2.1. Die Grundidee der von Craig favorisierten Referenztheorie besteht jedenfalls darin, dass Referieren als intentionale Aktivität von Personen zu verstehen ist, Referenz somit nicht als eine Beziehung, die unabhängig von diesen Intentionen zwischen Wörtern und außersprachlichen Objekten besteht. Es liegt nicht in der „Natur“ der Referenz, eine Wort-Welt-Relation zu sein. ${ }^{122}$ Wir können also singuläre Ausdrücke referierend verwenden, ohne uns mit ihnen auf irgendwelche außersprachlichen Objekte zu beziehen. So können wir beispielsweise Termini für Zahlen oder Mengen an Subjektstelle von Aussagen referierend verwenden, ohne dass wir uns darauf verpflichteten, die Existenz von Zahlen oder Mengen als Abstrakta anzuerkennen. Wir können reden, wie wir wollen, ohne uns auf irgendeinen Platonismus zu verpflichten.

${ }^{121}$ Van Inwagen u.a. 1998, 235f.

${ }^{122}$ Craig 2014, 119ff; ders. 2017, 16-20. 
Es soll hier nicht darum gehen, die sprachphilosophischen Gesichtspunkte des von Craig bevorzugten Referenzschemas zu untersuchen; auch nicht darum, ob seine ,intentionale Referenztheorie“ schwerwiegende Implikationen im Hinblick auf ontologische Voraussetzungen, Stichwort ,intentionale Objekte", hat. ${ }^{123}$ Versuchen wir vielmehr, in den Blick zu bekommen, welche Art von Ontologiekritik durch den Neutralismus vorgetragen wird. Zudem sollen uns auch in diesem Zusammenhang die Fragen beschäftigen, ob und unter welcher Rücksicht sie unsere Disziplin als Ganze betrifft bzw. ausschließlich einzelne Positionen. Last but not least: Welche Voraussetzungen macht Craigs Ontologiekritik?

Craig kritisiert zunächst jene Auffassung, der zufolge jede Verwendung von „es gibt“ zusammen mit einschlägigen Partikeln wie „etwas“ zum Anlass genommen wird, all das als Grundelement der Wirklichkeit anzunehmen, worauf sich letzterer Ausdruck bezieht. Eine solche Einstellung ginge Hand in Hand mit ontologisch „schweren“ Auffassungen bzgl. des Gebrauchs singulärer Termini an Subjektstelle. Craig kritisiert also die Ontologie, so könnten wir das im Hinblick auf das Thema dieses Abschnitts zusammenfassen, insofern sie Referenzschemata simplifizierend zur Ableitung ontologischer Verpflichtungen heranzieht. Das beträfe unsere Disziplin als Ganze, wenn jede Analyse der Grundelemente der Wirklichkeit und ihres Zusammenhangs auf eine derartige Auffassung der ontologischen Verpflichtung durch gebundene Variablen bzw. singuläre Ausdrücke hinausliefe. Wenn und insofern aber eine ontologische Position dem nicht näher tritt, und, selbst bei grundlegendem Bekenntnis zur Relevanz von Quantoren in ontologischen Untersuchungen bzw. zur Referenz von singulären Ausdrücken auf außersprachliche Objekte oder Referenten, eine kritische Analyse von „Es gibt...“-Aussagen, bzw. der „Natur“ der Referenzbeziehung (Craig) miteinbezieht, kann sie von Craigs Kritik nicht bzw. nicht mit voller Härte getroffen werden.

Das sollte für die Ontologie Perspektiven eröffnen, zumal wohl auch Craigs eigene Perspektive, der Neutralismus, nicht ohne kritikwürdige Voraussetzungen auskommt. Deflationismus und Meinongianimus wurden auf

\footnotetext{
${ }^{123}$ Chisholms ,intentionaler Zugang“ zur Ontologie ist beispielshaft dafür, dass gerade so einer extrem reichhaltigen Ontologie, gerade im Hinblick auf Abstrakta, Tür und Tor geöffnet wird. Vgl. u.a. ders. 1996.
} 
der metaontologischen Ebene bereits angeführt. Dazu kommt, dass auch der referenztheoretische Deflationismus nicht unproblematisch ist. Selbst wenn, nach diesem, Referenz keine Ontologie erfordert, so schließt sie, Referenz, auch keine kategorialen Schemata aus: Auch nach der von Craig vorgetragenen deflationistischen Referenztheorie kann sich ein Ontologe bzw. eine Ontologin mit stark realistischen Intuitionen mit singulären Ausdrücken auf platonische Entitäten beziehen; er bzw. sie muss das nur intendieren. Ein Blick auf Båve kann diese Kritik untermauern. So schreibt dieser: "[...] the deflationary theory of reference does not confine us to deflationary answers [...] on all questions concerning how we relate, mentally and linguistically, to the external world"124 bzw. "this theory does not force us to shun all questions about the relations between expressions and external objects“". ${ }^{125}$ Deflationäre Theorien der Referenz ,deflationieren“ Fragen nach dem sprachlichen Bezug zur externen Welt nicht; klarerweise auch nicht Fragen, welche Bewohner der externen Welt wir annehmen. Um es provozierend zu sagen: Auch mit Båve können wir gut und gern recht ,simpel“ Ontologie betreiben.

So lässt sich, vielleicht etwas zugespitzt, fragen, ob nicht auch Craigs Voraussetzungen unter bestimmter Rücksicht simplifizierend sind. Oder positiv zielorientiert: Kann man der maximalistischen Simplifizierung nicht durch moderatere Mittel beikommen als durch neutralistisch-deflationistische Interpretationen der Referenz aller singulären Termini bzw. durch leichtgewichtige Deutungen aller Quantoren? Kurzum: Craigs Ontologiekritik unter der Rubrik „simplifizierende Referenzschemata“ kann nicht nur auf bestimmte Positionen eingeschränkt, sondern auch kritisch analysiert werden im Hinblick auf ihre eigenen Voraussetzungen.

Die rhetorischen Fragen zu Beginn des letzten Absatzes mögen die Richtung andeuten, in der weiterführende Theorienbildungen erfolgen sollen. Hier wird uns jedoch weiter das Thema Simplifizierung unter der Rücksicht des Verstehens besonderer sprachlicher Funktionen beschäftigen. In diesem Sinne wollen wir uns nach Referenz bzw. Referieren, einem weiteren Anlassfall zuwenden, das ist Aussagen oder Prädizieren.

124 Båve 2009, 73.

125 Ebd., 71. 


\subsection{Simplifizierendes Verstehen des Prädizierens}

Ontologiekritik unter dem Vorzeichen einer simplifizierenden Deutung des Aussagens oder Prädizierens lässt sich pointiert bei John Heil festmachen. ${ }^{126}$ Die Punkte, auf die Heil abzielt, sind zum einen die grundsätzliche Unterscheidung zwischen aussagend gebrauchten sprachlichen Ausdrücken, also Prädikaten, und ontologischen Fakten, nennen wir sie der Einfachheit halber vorläufig Eigenschaften; bzw. zum anderen, dass es zwischen Prädikaten und Eigenschaften nicht in jedem Fall strukturelle Analogie gibt. Selbst wenn man also einen allzu einfachen nominalistischen Weg ablehnt und annimmt, dass Eigenschaften als Entitäten von prädizierend gebrauchten Ausdrücken zu unterscheiden sind, folgt daraus nicht, dass jedem wohlgeformten Prädikat „F“ beliebiger Komplexität auch tatsächlich eine strukturell analoge Eigenschafts-Entität F entspricht. Heils Anliegen ist es also herauszustreichen, dass man ontologische Fakten nicht rein aus einer Analyse von Prädikaten ersehen bzw. aus der grammatikalischen Struktur eines Prädikats keine ontologischen Strukturen ableiten könne. Kurzum: Nicht jedes Prädikat ist gleichsam ein Bild einer Entität der Kategorie der Eigenschaften.

Kritik übt Heil dementsprechend an einer Ontologie, die eine solche Strukturanalogie zwischen Prädikaten und Eigenschaften generell behauptet. Im Sinne der Themenstellung dieses Abschnitts können wir diese Kritik auch als Vorwurf einer Simplifizierung des Verstehens des Aussagens oder Prädizierens auffassen, in der Annahme, dass es durchaus im Bereich der Aufgaben unserer Disziplin liegt, auf das Verhältnis zwischen aussagend gebrauchten sprachlichen Ausdrücken und Entitäten, die in Trägern vorkommen oder inhärieren, zu reflektieren. Heil tritt dabei jedenfalls sehr resolut auf. Er erblickt in einer solchen Simplifizierung beispielhaft das Grundübel der „Ontologisierung“ einer Semantik. Dies aber führt unausweichlich zu einer ,myriad of philosophical puzzles“" ${ }^{\text {127, }}$, von denen gleich noch die Rede sein wird. Heil nennt die Behauptung einer solchen generellen Strukturanalogie zwischen Prädikaten und Eigenschaften in loser Anlehnung an Wittgensteins Tractatus auch ,picture-theory“. Diese Theorie gründet in einem (Korrespondenz-)

${ }^{126}$ Hier: Heil 2003. Vgl. dazu auch Kanzian 2016, III - 1.

${ }^{127}$ Heil 2003, 8. 
Prinzip, er nennt es „Ф“, das Heil so formuliert: „When a predicate applies truly to an object, it does so in virtue of designating a property possessed by that object and by every object to which the predicate truly applies (or would apply) ${ }^{6} .128$

Wir können uns hier nicht in die Details der Heil-Exegese begeben. $\Phi$ bedeutet als allgemeines Prinzip (und so ist es formuliert) jedenfalls, dass jedes Prädikat so zu deuten ist, dass eine ihm korrespondierende Entität jenem Objekt zukommt, von dem es ausgesagt wird. Es gibt nach $\Phi$ keinen anderen Weg, die ontologische Relevanz von Prädikaten zu interpretieren, als durch Verweis auf Entitäten, auch Heil spricht von Eigenschaften (engl.: properties), welche durch die Prädikate „designiert“ werden.

Ein offensichtliches Problem von $\Phi$ scheint zu sein, dass seine Annahme zu einem Dammbruch führt in Richtung unüberschaubar abundante Ontologie von Eigenschaften. Lässt man auf der sprachlichen Ebene etwa beliebig komplexe konjunkte und disjunkte Prädikate zu, was durchaus seinen Sinn hat, muss man nach diesem Korrespondenzprinzip wohl auch beliebig komplexe konjunkte und disjunkte Eigenschaften zulassen. Demnach kommen einem roten, runden Gegenstand nicht nur die Eigenschaften Rot bzw. Rund zu, sondern auch die Eigenschaften Rot-und-Rund bzw. Rot-oder-Grün bzw. Rund-oder-Eckig. Ähnliches ist bezüglich rein formaler Prädikate zu sagen. Behauptet man, dass etwas identisch sei mit sich selbst, müsste man nach $\Phi$ eine entsprechende Eigenschafts-Entität annehmen; ebenso wie, um es auf die Spitze zu treiben, die Eigenschaft, die darin besteht, die entsprechende Eigenschaft zu haben. Auch ein solches Prädikat lässt sich nämlich grammatikalisch korrekt bilden.

Eine weitere Problemgruppe macht Heil daran fest, dass es, relativ zu den verschiedenen Stufen von Prädikaten, nach $\Phi$ eine Hierarchie von Eigenschaften, folglich auch verschiedene Schichten der Wirklichkeit geben muss. Ein solches Viel-Schichten-Modell der Wirklichkeit aber eröffnet tatsächlich eine „myriad of philosophical puzzles“"129. Die Erklärung des Zusammenhangs und der Abhängigkeit der Schichten voneinander ist eines dieser puzz-

${ }^{128}$ Ebd., 26.

${ }^{129}$ Ebd., 28f. 
les, die Annahme einer basalen Ebene der Wirklichkeit, v.a. worin diese besteht, ein anderes.

Es kann hier nicht darum gehen, diesen Problemen weiter nachzugehen. So soll auch nicht behauptet werden, dass diese puzzles nicht doch gelöst werden könnten. Es geht, mit anderen Worten, (noch) nicht um die Negierung einer reichhaltigen Ontologie von Eigenschaften bzw. eines VielSchichten-Modells der Wirklichkeit. Worauf hier allerdings das Augenmerk gerichtet werden soll, ist, dass dies in der von Heil beanstandeten Ontologie nicht durch eine systematische Reflexion auf Existenz bzw. Existierendes angenommen und entwickelt wird, sondern eben in Folge eines Reflexionsabbruchs, der nach Maßgabe eigenen Anspruchs nicht erfolgen sollte, also simplifizierend im eingeführten Sinne ist.

Daraus ergibt sich bereits eine Antwort auf unsere standardmäßige Frage, welche Ontologie von Heils Kritik betroffen ist, welche aber nicht. Betroffen sind OntologInnen, die einer sehr reichhaltigen Ontologie von Eigenschaften bzw. einem „multy layer picture“ der Wirklichkeit das Wort reden und diese Reichhaltigkeit bzw. Vielschichtigkeit auf eine einfache Umlegung einer grammatikalischen Analyse von Prädikaten auf eine ontologische von Eigenschaften zurückzuführen. Legt man sich jedoch nicht auf $\Phi$ fest, steht es einem frei, die ontologische Relevanz von Prädikaten differenzierend zu verstehen; sprich nicht in allen Fällen so, dass den Dingen, von denen Prädikate ausgesagt werden, den Prädikaten korrespondierende Entitäten zukommen. Mit der Negierung von $\Phi$ schlagen wir jedenfalls Heils Weg ein, dessen Ausgang dieser wie folgt umschreibt: „We turn our backs on the idea that ontology can be settled by analysis“. ${ }^{130} \mathrm{Da}$ es solche Nicht- oder Anti- $\Phi$-Ontologien gibt, kann nicht die Ontologie als ganze Disziplin von Heils Kritik betroffen sein. Dabei wird angenommen, dass man eine differenzierende Sicht auf Eigenschaften entwickeln und ein nachvollziehbares Unterscheidungskriterium zwischen abundanten und nicht-abundanten Eigenschaften darlegen kann; ein Preis, der in einer systematischen Ontologie wohl nicht als zu hoch angesehen werden sollte. ${ }^{131}$

\footnotetext{
${ }^{130}$ Heil 2003, 9.

${ }^{131}$ In diesem Zusammenhang darf ich nochmals auf Kanzian 2016, III - 1, auch auf die dort angeführten Verweise, Bezug nehmen.
} 
Aus dem nunmehr abgehandelten Simplifizierungsvorwurf lässt sich also keine allgemeine Ontologiekritik ableiten. Würde dies behauptet, müsste man dies unter Heranziehung bestimmter, offensichtlich falscher Annahmen tun; dass man sich etwa in jeder Ontologie von Eigenschaften auf das von Heil inkriminierte $\Phi$-Prinzip verpflichtet. Das deckt sich im Ergebnis mit den anderen Ausprägungen einschlägiger Simplifizierungskritik (siehe 2.321 und 2.322), wobei die problematischen Voraussetzungen bei einem allgemeinen Missverstehen von sprachlichen Strukturen bzw. von Referenz, wie gesehen, anders gelagert sind. In diesem Sinn kann der erste Satz dieses Absatzes auch als allgemeines Resümee von Abschnitt 2.32 festgehalten werden.

\subsection{Simplifizierung als Einschränkung ontologischer Fragestel- lungen}

Wir kommen damit zu einem dritten Aspekt von Simplifizierung, welcher Anlass zu einer kritischen Einstellung gegenüber der Ontologie gegeben hat. Es ist die Ausblendung von Fragestellungen, die eigentlich im Bereich einer allgemeinen und universalen Theorie der Grundstrukturen der Wirklichkeit liegen müssen.

Die Wendung „eigentlich ... liegen müssen“ soll klarstellen, dies sei in diesem Zusammenhang nochmals angeführt, dass die Kennzeichnung als „allgemeine“ und „universale“ Theorie natürlich nicht ausschließt, dass es im Hinblick auf Grundstrukturen der Wirklichkeit auch außer-ontologische philosophische Themen gibt; etwa, wie in der Einleitung zu diesem Abschnitt angedeutet, im Umfeld von Fragen des erkenntnismäßigen Bezugs auf diese Strukturen. Die sind hier klarerweise nicht gemeint. Gemeint ist vielmehr eine von der Sache her ungerechtfertigte Ausblendung von Themen, im Zusammenhang mit vorschnellem Abbruch der Reflexion eben über die Grundstrukturen der Wirklichkeit.

Ein gemeinsamer Nenner der unter dieser Rücksicht vorgetragenen Ontologiekritik besteht darin, dass eine Ontologie dann zu kurz greift, eben simplifizierend im nunmehr gemeinten Sinn vorgeht, wenn sie sich rein auf Existenzfragen beschränkt. Bei einer solchen Beschränkung bleibt außer Acht, dass eine Theorie der Grundstrukturen der Wirklichkeit nicht nur die Erarbeitung kategorialer Schemata (Es existieren Vorkommnisse der Katego- 
rien $\mathrm{A}, \mathrm{B}, \mathrm{C}$; nicht aber Ds, Es, Fs ...) umfassen darf, sondern a) auch die Verhältnisse von Vorkommnissen unterschiedlicher Kategorien klären muss. Weiterführend wäre in diesem Zusammenhang die Frage, ob sämtliche Elemente der Grundstrukturen Entitätenstatus aufweisen, oder ob nicht auch formale bzw. epiphänomenale Elemente eine unverzichtbare Rolle spielen. Damit hängt b) die Frage der Unterscheidung zwischen grundlegenden bzw. gegründeten (engl.: grounded) Kategorien zusammen. Schließlich wird in diesem Kontext immer wieder auch c), die Frage nach der Revidier- und Entwickelbarkeit ontologischer Theorien genannt.

Den ersten Gesichtspunkt a) bringt wiederum Peter Simons auf den Punkt, wenn er bekundet: „A mere list of categories is not enough [... ${ }^{“ .}{ }^{132}$ Wie im letzten Absatz angesprochen, genügt es für eine Theorie der Grundstrukturen der Wirklichkeit nicht, eine einfache Liste dessen zu erstellen, was man denn als existierend akzeptiert oder auch nicht; etwa anzugeben, dass man zwar Substanzen, Eigenschaften, vielleicht auch noch Ereignisse als Entitäten anzunehmen geneigt ist, nicht jedoch abstrakte Individuen wie mathematische Gegenstände o.ä.. Dementsprechend fährt Simons an der zitierten Stelle auch fort , ,[...] they must be put to work in general metaphysical statements describing the relations of things in the categories to one another and the general metaphysical principles governing their employment". ${ }^{133}$ Es braucht zumindest noch Reflexionen über das Verhältnis der angenommenen Kategorien von Entitäten, am besten entwickelt aus Grundsätzen, die darüber Aufschluss geben, wie die Vorkommnisse einzelner Kategorien im Hinblick auf eine Gesamttheorie der Grundstrukturen der Wirklichkeit zu interpretieren sind. Wie verhalten sich z.B. Substanzen zu Eigenschaften, bzw. wie kann man diese als Elemente der Wirklichkeit verstehen? Was bedeutet es überhaupt für Substanzen und Eigenschaften, Grundelemente der Wirklichkeit zu sein?

Das eben angeführte Beispiel soll keinesfalls insinuieren, dass Simons in besagtem Artikel substanzontologische Ambitionen zeigt. Er meint vielmehr in Whiteheads Prozessontologie eine geradezu paradigmatische Vorlage für

132 Simons 1998b, 386.

${ }^{133}$ Ebd. 
gelungene ontologische Systematik vorzufinden. Das soll hier nicht Gegenstand der Erörterung sein. Maßgeblich sind jedoch weitere von Simons angeführte Charakteristika einer solchen Systematik, die über das blanke Behaupten der Existenz von Vorkommnissen bestimmter Kategorien hinausgehen: die Unterscheidung zwischen formaler und materialer Ontologie, die Verbindung bzw. die Abhängigkeit von Vorkommnissen unterschiedlicher Kategorien, schließlich die Frage nach dem Letzten (engl.: the ultimate) als dem Prinzip ontologischer Systematik.

Bzgl. der Differenzierung zwischen formaler und materialer Ontologie nimmt Simons auf Husserl Bezug, insofern dieser zwischen der Frage nach einem kohärenten allgemeinen Kategorienschema und der Frage nach Charakteristika besonderer Vorkommnisse unterscheidet. Materiale Ontologie bringt Simons auch in Zusammenhang mit Wolffs metaphysica specialis bzw. dem Anliegen regionaler Ontologien, etwa von Ontologien des Lebendigen oder des Sozialen. ${ }^{134}$ Es sei dahingestellt, ob es nicht treffendere Unterscheidungskriterien zwischen formaler und materialer Ontologie geben mag. ${ }^{135}$ Entscheidend ist, dass die mit der Differenzierung vorliegenden Themen jedenfalls Gegenstand ontologischer Reflexion zu sein haben.

Im Zusammenhang mit dem Punkt „Verbindung bzw. Abhängigkeit von Vorkommnissen unterschiedlicher Kategorien“ betont Simons das Erfordernis ,,anatomizing the differences between the categories“. ${ }^{136}$ Die Unterscheidungen zwischen den Kategorien müssen wir, um Simons wiederum frei zu interpretieren, mit begrifflichem Seziermesser vornehmen. Tun wir das, können wir elementare Tatsachen (engl.: elementary facts) aufweisen, die im Vorfeld der Zuweisung von Entitäten in Kategorien eine Rolle spielen. Bemerkenswerterweise spricht Simons davon, dass diese Fakten selbst nicht

\footnotetext{
${ }^{134}$ Simons 1998 b, 380.

135 Z.B. Roman Ingardens Vorschlag, zwischen existential-, formal- und materialontologischen Fragen zu unterscheiden. Daraus geht zum einen klar hervor, dass sich die Ontologie gerade nicht auf Existenzfragen beschränken lässt; zum anderen, dass man „,materiale Ontologie“ als allgemeine Theorie „,bestimmter Gegenständlichkeit“ (Ingarden) verstehen kann, nicht „,regional“ eingegrenzt. Vgl. Ingarden 1964, 58ff.

136 Alle Bezüge auf Simons in diesem Absatz referieren auf Simons 1998b, 381f. Auch Keinänen hat das Thema ,exakte Analyse des Verhältnisses von Vorkommnissen unterschiedlicher Kategorien“ als wesentlich für die Ontologie, folglich als eines ihrer Qualitätskriterien aufgegriffen: vgl. Keinänen 2008, 46.
} 
Klassen konkreter Entitäten ausmachen, sondern vielmehr ,analytic invariants discernible behind such classes“ seien. Das entspricht der vorab genannten Idee, dass bei einer Theorie der Grundstrukturen der Wirklichkeit nicht nur Entitäten eine Rolle spielen, sondern auch ,formale“ Elemente, die hinter oder „unter" kategorialen Schemata liegen, für diese jedenfalls grundlegend sind. Als Beispiel nennt Simons an dieser Stelle übrigens ontologische Abhängigkeit (engl.: ontological dependence), insofern sie nicht wieder als zweistellige Relation mit Entitätenstatus zu verstehen ist, sondern als grundlegendes Charakteristikum des Verhältnisses von Vorkommnissen unterschiedlicher Kategorien, mit dessen Hilfe auch die Eigenart dieser Vorkommnisse ontologisch analysiert werden kann. Dieser Nicht-Entitätenstatus von Grundlegendem lässt sich bei Simons (im Anschluss an Whitehead) auch auf „das Letzte“ (engl.: the ultimate) beziehen. ${ }^{137}$ Es stellt ein finales Prinzip dar, aus dem sich eine Systematik ontologischer Theorienbildung herleiten lässt, somit selbst nicht Element dieser Systematik sein kann. Simons stellt hier Bezüge zur vorsokratischen Philosophie (Anaximander, Anaximenes) her, insofern sie nach der ,,arché“ frägt und aus einem grundlegenden Prinzip (apeiron/Unbestimmtes, aer/Luft) die Vielheit verschiedener Elemente der Wirklichkeit zu erklären sucht. Dies braucht uns hier ebenso wenig zu beschäftigen wie Whiteheads „creativity“ als ein solches Letztes. Festzuhalten bleibt, dass man auch von diesem Gesichtspunkt aus das Erfordernis festmachen kann, dass mit einer einfachen Auflistung von Kategorien das Geschäft der Ontologie keinesfalls erledigt ist; bzw. dass sich die Ontologie dann einer fundamentalen Kritik ausgesetzt sieht, wenn sie sich in simplifizierender Weise darauf beschränkt.

Daraus ergibt sich ein nahtloser Übergang zum zweiten eingangs geschilderten Gesichtspunkt, b), thematischer Simplifizierung, der im Ignorieren der Unterscheidung zwischen grundlegenden bzw. gegründeten (engl.: grounded) Kategorien besteht. Den nunmehr gemeinten Aspekt kann man in einem allgemeinen Sinn verstehen als Frage, ob sich aus den Verhältnisbestimmungen zwischen Kategorien nicht eine gewisse Hierarchisierung von Entitäten ergibt, die von der Ontologie als genuines Thema aufgefasst werden muss. Ge-

${ }^{137}$ Simons 1998b, 387f. 
rade in neuester Zeit hat sich in diesem Zusammenhang eine speziellere Debatte in der (Meta-)Ontologie etabliert, die mit dem Stichwort „Gründung“ überschrieben werden kann: Haben die besagten Verhältnisbestimmungen nicht eine innere Systematik? Dergestalt, dass sich die verschiedenen Verhältnisse aus einem Prinzip heraus verstehen und auch differenziert darlegen lassen? Auf unseren Kontext bezogen: Kann es sich die Ontologie leisten, einem solchem Prinzip nicht nachzugehen?

Jonathan Schaffer war maßgeblich an der Etablierung dieser GründungsDebatte beteiligt. Für unsere Zwecke mag es zunächst genügen, auf seinen Beitrag „On What Grounds What“ zu verweisen, ${ }^{138}$ in dem die letztere Frage des vorhergehenden Absatzes gleich in aller Deutlichkeit beantwortet wird. Existenzfragen seien, so Schaffer, eigentlich trivial. In diesem Sinn vertritt Schaffer eine Art Permissivismus (engl.: permissivism), dem zu Folge alles Nichtwidersprüchliche im Wertebereich des Existenzquantors anzunehmen sei. ${ }^{139}$ Beschränkte sich die Ontologie rein auf Existenzfragen, reduziere sie sich auf Triviales. Damit wäre der Simplifizierungsvorwurf radikalisiert. Schaffer plädiert dementsprechend für eine Ontologie, die jenes Anliegen aufgreift, das er seit Aristoteles als ihre eigentliche Fragestellung ausmacht: „What grounds what?“140 Schaffer sieht es nämlich als den Kern der aristotelischen Kategorienlehre an, die unterschiedlichen Weisen der Abhängigkeit von Vorkommnissen gegründeter Kategorien von der Gründungskategorie, den Substanzen, darzulegen : ,[...] categories just are ways things depend on substances". 141

Diese Formulierungen stellen auch den Zusammenhang mit der vorhin erwähnten aktuellen Debatte der Gründung her. Schaffer ist unter dieser Rücksicht richtungsweisend, als er in besagtem Artikel wesentliche Elemente von ,grounding“ darlegt. Zunächst betont er, dass es sich dabei um einen

\footnotetext{
138 Hier Schaffer 2009. Einen eingehenderen Überblick über die besagte Debatte bietet Correia/Schnieder 2012. In den dort enthaltenen Beiträgen wird klar, dass der Sachgehalt der Gründungsdebatte natürlich nicht neu erfunden ist, ihre systematische Entfaltung jedoch einiges an Potential aufweist, gerade im Hinblick auf das Verstehen der Verhältnisse unterschiedlicher Kategorien. Kritik an „Gründung“ übt u.a. Koslicki 2012.

${ }^{139}$ V.a. Schaffer 2009, 356ff. Als Ausnahme führt Schaffer, ebd. 359, übrigens meinongianisch subsistierende Gegenstände an.

${ }^{140} \mathrm{Vgl}$. Schaffer 2009, 350f.

${ }^{141}$ Vgl. Schaffer 2009, 355, Hervorhebung Schaffer.
} 
Grundbegriff handelt, der selbst nicht wieder einer ontologischen Analyse zugänglich ist. In diesem Sinne ist ,grounding“ jedenfalls einfach (engl.: primitive). Schaffer bedient sich hier sogar eines Superlativs, in dem er grounding als den Strukturbegriff der Metaphysik überhaupt kennzeichnet. ${ }^{142}$ Insbesondere lehnt es Schaffer ab, Gründung über „Supervenienz“ zu analysieren, wie das eine Zeitlang in manchen ontologischen Kreisen durchaus üblich war. ${ }^{143}$

Als ein solcher einfacher Begriff kommt „grounding“ in der Analyse anderer Begriffe vor, welche die Verhältnisse zwischen unterschiedlichen Kategorien beschreiben, etwa „fundamental für“ bzw. „abgeleitet aus“ zu sein. Um beim Beispiel aristotelischer Ontologie zu bleiben, könnte man sagen, dass es sich bei Akzidentien um abgeleitete, d.h. gegründete, bei Substanzen aber um fundamentale, d.h. nicht-gegründete Entitäten handelt.

Seine Einfachheit schließt nicht aus, dass man „Gründung“ formal explizieren und im Hinblick auf besondere Verhältnisse differenzierend darlegen kann. Charakteristische formale Merkmale für Gründung sind jedenfalls $I r$ reflexivität, Asymmetrie und Transitivität. ${ }^{144}$ Daraus ergibt sich, dass sich keine Entität selbst gründen kann und auch wechselseitige Gründung ausgeschlossen ist. Ein von $\mathrm{x}$ gegründetes $\mathrm{y}$ kann jedoch selbst wiederum ein $\mathrm{z}$ gründen, was $\mathrm{z}$ auch als Gründung von $\mathrm{x}$ ausweist. Dass dies Raum für die Darlegung differenzierender Abhängigkeitsverhältnisse eröffnet, kann man ebenfalls anhand der aristotelischen Kategorienlehre ersehen: So wird wohl die Gründung von Substanzen im zweiten Sinn durch Substanzen im ersten Sinn anders zu verstehen sein als das Verhältnis von Substanzen im ersten Sinn zu Vorkommnissen diverser akzidenteller Kategorien.

Ausgehend von seinen Grundannahmen versucht Schaffer in besagtem Artikel auch noch eine Art Neukonzeption aristotelischer Ontologie unter naturalistischem Vorzeichen. Demzufolge gibt es als die (einzige) Substanz den

\footnotetext{
${ }^{142}$ Vgl. Schaffer 2009, 364. Seine Formulierung im Original: ,it [grounding] is the primitive structuring conception of metaphysics". Hervorhebung Schaffer

${ }^{143}$ Ebd.

${ }^{144}$ Schaffer 2009, 376. Diese formalen Charakteristika sind in der Gründungsliteratur allgemein anerkannt: siehe dazu auch die Einleitung zu Correia/Schnieder 2012, Grounding: an opinionated introduction, u.a. 4,8 .
} 
Kosmos, verstanden als universale physikalische Größe. ${ }^{145}$ Der Kosmos gründet sämtliche seiner Bestandteile in deren Existenz. Es kann hier nicht die Aufgabe sein, diese eher spinozistisch anmutende Variante einer Substanzontologie näher zu erörtern; ebenso wenig wie eine Diskussion von Schaffers Annahme, dass nicht nur das Gründende und das Gegründete existiert, sondern genau so auch die Gründung als zweistellige Relation. ${ }^{146}$ Worum es geht, ist Simplifizierung. Und die liegt, und das ist mit Schaffer klar darzulegen, dann vor, wenn man sich in der Ontologie rein auf Existenzfragen beschränkt, und das Anliegen der mit „Gründung“ verbundenen Systematik außer Acht lässt.

Es bleibt nunmehr der eingangs angeführte Gesichtspunkt c) möglicher Simplifizierung, das Ausblenden der grundsätzlichen Revidier- und Entwickelbarkeit ontologischer Theorien.

Wie einige andere Aspekte der Ontologiekritik bedarf auch c) anfänglicher Präzisierung, um nicht Anlass zu schwerwiegenden Missverständnissen zu geben. So bedeutet die hier gemeinte Revidier- und Entwickelbarkeit ontologischer Theorien nicht, dass man jede These über die Grundstrukturen der Wirklichkeit unter relativistischen Generalverdacht stellt. Ontologische Theorien haben keinen Kultur- oder Zeitindex, etwa derart, dass ihre Geltung von vornherein auf bestimmte gesellschaftliche oder historische Gegebenheiten beschränkt wäre. Gerade wenn man jedoch, wie hier geschehen, Ontologie als systematische Reflexion versteht auf Voraussetzungen menschlicher Praxis bzgl. Existierendem, sei diese Praxis alltäglich, sei sie speziell, wie im Falle wissenschaftlicher Tätigkeit, kommt man nicht umhin, bei der ontologischen Theorienbildung auch die mögliche Varianz dieser Praxis mit einzubeziehen. Dementsprechend wird das einschlägige Nachdenken über diese Praxis bzw. die Darlegung ihrer ontologischen Rahmenbedingungen den Aspekt

\footnotetext{
145 Schaffer 2009, 378f.

${ }^{146}$ Ebd., 353. Diese These ist wohl nur im Kontext von Schaffers maximalistischem Permissivismus zu verstehen. Fasst man, unabhängig davon, „Gründung“ als den metaphysischen Strukturbegriff auf, ist er also Prinzip ontologischer Systematik, kann er nicht selbst Element dieser Struktur bzw. Teil besagter Systematik sein. Gründung kann keine Entität sein, wie das u.a. Jonathan Lowes Einsicht entspricht, dass grundlegende Strukturbegriffe, Lowe nennt sie ,formal“, ,too fundamental“ seien, ,to be something in the world - an element of being because it is that without there could be no beings and so no world" (Lowe 2006, 49).
} 
der Weiterentwicklung zu berücksichtigen haben. Das schließt nicht aus, dass das Ergebnis dieses Nachdenkens einen Kern invarianter Elemente der ontologischen Rahmenbedingung jedweder Praxis beinhaltet. Ontologie kann sich nur nicht von vornherein der Reflexion über diesen dynamischen Aspekt ihrer Theorienbildung verschließen; sei es dadurch, dass sie entwickelbare Praktiken als für sie irrelevant einschätzt, sei es, dass sie die Konsequenzen der zugestandenen Relevanz nicht in ihren Ansatz zu integrieren vermag.

Wir können uns wieder auf Peter Simons berufen, um diesen Aspekt der Ontologiekritik in der aktuellen Debatte zu verankern. Simons kritisiert die Ontologie, insofern sie ihre Theorien „stable, invariant across cultures and without significant history or change" darlegt. ${ }^{147}$ Simons verweist darauf, durchaus im Sinne der Ausführungen im letzten Absatz, dass sich ontologisch relevante Praktiken ändern. Simons bezieht sich dabei zwar ausschließlich auf die Naturwissenschaft und deren Ergebnisse, den oben angeführten Punkt illustriert er damit aber allemal: Eine Ontologie, die sich von vornherein als revidier- und entwicklungsresistent erweist, kann die nicht-stabile, variante Dimension einschlägiger Praxis nicht erreichen. Ins selbe Horn hat in einem älteren Beitrag Susan Haack gestoßen, wenn sie die Ontologie kritisiert, insofern sie unter der Voraussetzung agiert, einen zeitlich und interlinguistisch invarianten Rahmen bzgl. Existierendem zu entwerfen, der als Voraussetzung individueller bzw. sozialer Praxis angenommen wird. ${ }^{148}$ Dies wird der Dynamik dieser Praktiken nicht gerecht.

Auch hier würde es unseren Rahmen sprengen, die Details der Theorienbildung unserer ReferenzautorInnen zu erörtern, zumal das, zumindest im Falle von Simons, bereits geschehen ist. Unser Augenmerk an dieser Stelle ist Simplifizierung. Und die liegt dann vor, wenn eine Ontologie, von ihrem Ansatz her, die Reflexion über die Varianz ihrer Theorienbildung ausblendet.

Wir können damit wieder zur obligaten Frage kommen nach den Voraussetzungen der einschlägigen Ontologiekritik, das ist Simplifizierung unter der Rücksicht einer ungerechtfertigten Einschränkung von Themenstellungen. Diese wurden im Zuge der Ausführungen in diesem Abschnitt bereits ange-

\footnotetext{
${ }^{147}$ Simons 1998b, 379.

${ }^{148}$ Vgl. Haack 1978, u.a. 81, 86, 90.
} 
führt und sollen hier deshalb nur noch stichwortartig wiederholt werden: So wird bei Schaffer die Kritik an einer Einschränkung der Ontologie auf Existenzfragen bzw. auf eine simple Auflistung von Kategorien dadurch begründet, dass Existenzfragen als solche eigentlich trivial seien. Schaffer macht damit also eine nicht unwichtige Voraussetzung, die er auch dankenswert explizit darlegt als Bekenntnis zu einem ontologischen Permissivismus. Es sei jedoch in diesem Fall betont, dass sich die von ihm geäußerte Simplifizierungskritik auch unabhängig von diesen seinen $\left(\right.$ starken $^{149}$ ) Prämissen darlegen lässt. So führt Schaffer selbst Aristoteles und die von ihm inspirierte Tradition an. Nach dieser sind Existenzfragen, sofern sie als solche artikuliert werden, aber keinesfalls trivial. Dennoch ist gerade die aristotelische Metaphysik oder Ontologie ein geradezu paradigmatisches Beispiel dafür, dass es eben mit einer simplen Auflistung von obersten Gattungsbegriffen, unter die alles Seiende fallen mag, nicht getan ist. Als weitere Beispiele können wir Husserl und seine NachfolgerInnen anführen, die ja auch von Peter Simons erwähnt werden. Unabhängig von Schaffers Voraussetzungen würden „HusserlianerInnen“ jedwede Beschränkung der Ontologie etwa auf eine Auflistung dessen, was es gibt oder existiert, als inakzeptabel abtun. Kurzum: Der Vorwurf wird faktisch unter verschiedenen Voraussetzungen und Prämissensystemen konsistent vertreten, sodass man nicht davon sprechen könnte, dass die Anführung einschlägiger Kritik in irgendeiner Weise auf eine Gruppe solcher Voraussetzungen verpflichtete. Das gilt gleichermaßen für Peter Simons, der seine Kritik mangelnder ontologischer Systematik faktisch unter dem Vorzeichen des Naturalismus darlegt, insbesondere in Anwendung von Whiteheads Theorienbildung. Auch hier können wir festhalten, dass Simons' Anliegen gleichermaßen vor anderen theoretischen Hintergründen vertreten werden kann.

Um auch die zweite standardmäßige Frage im Kontext unserer Analyse von Ontologiekritik zu platzieren, können wir dem nachgehen, gegen wen, das heißt gegen welche Ontologie, die einschlägige Kritik der Simplifizierung gerichtet ist. Es liegt auf der Hand: Keiner bzw. keine der zitierten KritikerInnen hat die Ontologie als Ganze vor Augen. Die Behauptung, dass die Ontologie als philosophische Disziplin zu kritisieren wäre, weil sie perma-

${ }^{149}$ Vgl. die kritische Analyse von permissivistischen Prämissen u.a. in Hewitt 2015. 
nent Themen ausblendet, die eigentlich in ihrem Bereich liegen, wäre offensichtlich überzogen bis absurd. Es geht, wie eigentlich bei den meisten anderen Stoßrichtungen der Ontologiekritik, um bestimmte Tendenzen in der Ontologie bzw. um konkrete Weisen, unsere Disziplin zu betreiben. Simons und Haack haben v.a. deskriptive Ontologie im Stile Peter Strawsons vor Augen. Das ist in diesem Kontext zur Kenntnis zu nehmen und an gegebener Stelle näher zu untersuchen. Schaffer hingegen hat zunächst Quine als „Simplifizierer" in Verdacht, der mit seinem programmatischen Credo zu Beginn von „On What There Is“, dass es der Ontologie eben um alles gehe, samt der Durchführung des Programms, durchaus unter dem zur Debatte stehenden Gesichtspunkt zu hinterfragen ist. ${ }^{150}$ Einschlägige Quine-Kritik hat neulich auch Kathrin Koslicki in ihrem Artikel „Questions of Ontology“ vorgetragen, und diese Kritik auch gleich auf Carnap angewendet. Insofern beide die Ontologie auf Existenzfragen und ihre Interpretation beschränken, legt sich jene kritische Einstellung nahe, die Koslicki so formuliert: „But one may justifiably wonder whether the Quinean and Carnapian perspective on ontology really does justice to many of the most central concerns of this discipline“" ${ }^{15}$

In diesem Artikel führt die Autorin Fragen nach ontologischer Abhängigkeit bzw. Fundamentalität als solche „,most central concerns“ der Ontologie an. ${ }^{152}$ Der Verweis auf Carnap möge in diesem Zusammenhang jedenfalls das Augenmerk darauf lenken, dass auch manche VertreterInnen des Deflationierungsprogramms, insofern sie sich in ihrer Positionierung explizit auf Carnap berufen, von dieser Kritik betroffen sind. Insbesondere die Easy Ontology, aber durchaus auch die Modest Ontology (siehe Abschnitte 2.12 bzw. 2.22) sind unter dieser Rücksicht zu hinterfragen: Wird bei der Fokussierung auf Existenz bzw. auf Interpretationen von Existenzfragen nicht ungerechtfertigterweise auf Themen vergessen, die eigentlich im Bereich ontologischer Untersuchungen liegen sollten?

Auch dies soll an dieser Stelle nicht abschließend beantwortet werden. Der Hinweis darauf, dass unter dem Stichwort „Simplifizierungsverdacht“

\footnotetext{
${ }^{150} \mathrm{Vgl}$. Schaffer 2009, 348f.

151 Koslicki 2016, 222.

152 Aufhänger ist v.a. die unterschiedliche Interpretation partikularer Eigenschaften in Tropen- (Campbell 1990) bzw. Substanz-Ontologien (Lowe, u.a. 1998).
} 
nun auch Positionen im Bereich des Naturalismus und des Deflationismus ins Visier der Ontologiekritik geraten, soll jedoch unterstrichen sein. Und zwar unter der Rücksicht, dass verschiedene Varianten der Ontologiekritik offensichtlich nicht nur im positiven Anliegen in einem inneren Zusammenhang stehen. Das wurde im Zuge der vorliegenden Ausführungen immer wieder hervorgehoben. Einzelne Stoßrichtungen der Ontologiekritik stehen auch in einem wechselseitigen Kritikverhältnis. ${ }^{153}$ Auch wenn es nicht möglich ist, hier eine vollständige Darlegung aller dieser wechselseitig kritischen Verweisungszusammenhänge zu erzeugen, oder gar eine Begründung zu versuchen, welcher Kritik unter welcher Rücksicht nun recht zu geben ist - wir halten fest: Eine Analyse des Referenten der Simplifizierungskritik an der Ontologie zeigt, dass hier nicht nur ontologische, sondern auch andere ontologiekritische Positionen betroffen sind.

Damit können wir überleiten zu einer resümierenden Zusammenfassung der „Bedenken gegen die Ontologie aus der aktuellen Metaontologie“, also jener Ontologiekritik, die hier beispielhaft berücksichtigt werden konnte.

\subsection{Ontologiekritik: ein Resümee}

Ein erster Eindruck, den die Leserin bzw. der Leser dieses zweiten Kapitels gewonnen haben sollte, ist, dass die Ontologiekritik der gegenwärtigen Metaontologie ein komplexes, uneinheitliches, aber auch vielschichtiges Phänomen ist. Dieser Eindruck mag nunmehr zusammenfassend unterstrichen werden. Es sei zunächst betont, dass mit der (nicht exhaustiv gemeinten) Unterteilung der Kritik in Deflationierung, Naturalisierung, Simplifizierung versucht wurde, eine gewisse Systematisierung zu erzeugen. Dadurch sollte allerdings der gewünschte Ersteindruck nicht geglättet werden. Dem entspricht zunächst, dass eine gewisse Uneinheitlichkeit in der Präsentation der gewählten Positionen beibehalten wird: So ist manche Theorie als Träger der Ontologiekritik, gleichsam als Subjekt, dargestellt - z.B. die Easy Ontology; man-

\footnotetext{
153 Paradigmatisch kann hier auch Thomas Hofwebers Kritik an der Gründungsdebatte erwähnt werden. Diese sei, wie wir im Abschnitt 2.22 gesehen haben, symptomatisch für „nicht-bescheidene“, gar „esoterische“ Metaphysik. Es kann also nicht nur die „Modest Ontology“ als Fortführung von Carnaps Programm von VertreterInnen der Relevanz von grounding unter der Rücksicht Simplifizierung kritisiert werden. Die Kritik wird auch erwidert.
} 
che aber als Referent der Kritik, also als Objekt, behandelt - z.B. modellierende Ontologie oder der Maximalismus.

Eine weitere Ebene bewusst beibehaltener Diskrepanz beruht auf der unterschiedlichen Reichweite von Ontologiekritik. Bei manchen Angriffen ist tatsächlich die Ontologie als Ganze, sprich als gesamte philosophische Disziplin anvisiert. Denken wir an manche extremen Ausformungen der Streitum-Worte-Partei, an die Metaphysikkritik der frühen Analytischen Philosophie, aber auch an Gilbert Ryle in seiner radikalen Theorie der Varianz äquivoker Quantorenbedeutungen. Dabei geht es nicht um die Kritik an einzelnen ontologischen Positionen, sondern vielmehr darum, die kognitive Irrelevanz, Trivialität, ja Sinnlosigkeit unserer Disziplin insgesamt zu erweisen. Davon zu unterscheiden ist Ontologiekritik, die sich an bestimmten Zugangsweisen zur Ontologie stößt. Die Kritik etwa an deskriptiver Ontologie bzw. an einer sich am common sense orientierenden Theorienbildung, wie sie beispielsweise von Peter Simons vorgetragen wird, sei unter dieser Rücksicht angeführt. Ebenso kann Thomas Hofwebers Zurückweisung von unbescheidener bzw. esoterischer Ontologie hier eingeordnet werden. Vergleichbar damit ist auch solche Ontologiekritik, die im Kern auf eine bestimmte These abzielt. Hier können wir Craigs Anti-Platonismus anführen, ebenso wie Heils Verdikt gegen allzu einfache Deutungen der Prädikation durch so manche ontologische Theorie. Jedenfalls, und das mag als ein Ziel der Darstellung herausgestrichen sein, tut man gut daran, Ontologiekritik im Hinblick auf die angezielte Reichweite zu befragen. Dieses Anliegen wird dadurch nicht gemindert, dass besagte Reichweite mitunter nicht ganz klar festzumachen ist: So kann aus einer partiellen Ontologiekritik durchaus eine universale werden; wenn sich etwa herausstellt, dass die angezielte Rücksicht bzw. die kritisierte Detailthese unsere Disziplin in einem ihrer Grundanliegen betrifft. Bei Hofweber mag sich dieser Verdacht durchaus einstellen. Wenn man bedenkt, dass jenes Refugium an Restrelevanz, das er der Ontologie zubilligt, zu wenig ist, um als selbständige philosophische Disziplin zu überleben. Aber auch das Umgekehrte mag der Fall sein: Wenn sich eine vermeintliche Fundamentalattacke auf die Ontologie auf den zweiten Blick doch als Angriff auf bestimmte Weisen, Ontologie zu betreiben, erweist. Hier können wir die Kritik des frühen Carnap anführen. Betrifft sein Sinnlosigkeitsvorwurf jede Ontolo- 
gie, oder vielleicht doch nur jene Varianten, die er anfangs der 30er Jahre des 20. Jahrhunderts vor Augen hatte?

Eine weitere Ebene der Unterscheidung von Ontologiekritik betrifft die vielfältigen Voraussetzungen, die bei den jeweiligen Angriffen gemacht werden. Diese sind naturgemäß äußerst verschieden und an manchen Stellen auch nicht immer transparent. Umso wichtiger erscheint es, diese durch gezielte Analyse ins Auge zu bekommen und in eine systematische Beurteilung der einzelnen Kritik einfließen zu lassen. Die jeweiligen Prämissen müssen an dieser Stelle nicht nochmals aufgelistet werden, zumal das in den jeweiligen Abschnitten stets zum Pflichtprogramm gehört hat. Resümierend können wir jedoch festhalten, dass sich diese Voraussetzungen mitunter nicht nur als umfangreich, sondern auch als schwer und schwerwiegend herausstellen. Denken wir in diesem Zusammenhang etwa an Siders letztlich doch recht markanten Physikalismus, den er im Schlusskapitel von ,Writing the Book of the World" als Voraussetzung seiner oft sehr speziellen Thesen darlegt. Beachten wir, um beim Thema zu bleiben, jene gar nicht selbstverständliche Verhältnisbestimmung von Philosophie und Naturwissenschaft, wie sie Naturalismus und Physikalismus, insbesondere ihrer Ontologiekritik, zugrunde liegt. Denken wir aber auch an Hirschs Prämisse der theoretischen Unentscheidbarkeit der primären Bedeutung des Existenzquantors, die ihm Anlass zur Kritik an einer Ontologie der hyper-theoreticalness gibt. Schaffers Permissivismus wurde eben erst als schwere Vorannahme einer speziellen Ontologiekritik dargestellt.

Damit soll keineswegs behauptet werden, es sei anrüchig, in der Entwicklung von kritischen Theorien auf bestimmten Voraussetzungen aufzubauen. Das wäre grober Unsinn. Es sei lediglich darauf hingewiesen, dass es nicht nur legitim, sondern auch angebracht ist, ontologiekritische Positionen auf solche Vorannahmen hin zu analysieren. Das hilft nicht nur, die Kritik zu verstehen, sondern auch, sie für die Ontologie selbst fruchtbar zu machen. Wenn nötig kann dies dazu beitragen, die Ontologie als Ganze bzw. in einer ihrer Zugangsweisen zu verteidigen - gerade dann, wenn sich Einwände als Fundamentalkritik verstehen, ihre Voraussetzungen aber höchst problematisch sind. 
Unter apologetischer Rücksicht kann auch darauf aufmerksam gemacht werden, dass sich unter den Voraussetzungen ontologiekritischer Positionen mitunter sehr konkrete Annahmen über die Grundstrukturen der Wirklichkeit befinden. So haben wir bei Theodore Sider gesehen, dass die Unterscheidung zwischen substantiellen und nicht-substantiellen Fragen durchaus auf vorab angenommenen besonderen Prämissen, etwa einem spezifischen Vierdimensionalismus beruht. Etwas allgemeiner ist der Hinweis, dass der Versuch der Deflationierung der Ontologie durch Rückführung auf einen Streit um Worte, wie gesehen, handfeste ontologische Überlegungen und Dispute voraussetzt, um stimmig vorgetragen werden zu können. Pointiert gesagt: Manche Ansätze, die Ontologie zu kritisieren, setzen sie im Grunde voraus.

Bemerkenswert, gerade im Hinblick auf die Perspektiven einer zukünftigen Ontologie, ist auch, dass sich manche ExponentInnen der Ontologiekritik in der Stoßrichtung ihrer Kritik überschneiden. Das haben wir in besonderer Weise bei Deflationierung und Naturalisierung gesehen. Wie zuletzt angesprochen, können wir bei verschiedenen ontologiekritischen Ansätzen aber auch wechselseitig kritische Verhältnisse feststellen: So kritisiert Amie Thomassons Easy Ontology Quine. Quine kritisiert geschichtswirksam Carnap, Carnap bzw. neo-carnapianische Positionen aber den Realismus. Sider, als Realist, wiederum kritisiert die Easy Ontology grundsätzlich, womit sich der Kreis schließt. Diese und ähnliche Verhältnisse sagen nichts über die Legitimität und Adäquatheit der einzelnen Argumentationslinien aus. Konkrete Ontologiekritik wird dadurch nicht widerlegt. Aus innerontologiekritischen Widerlegungskreisen zeigt sich aber besonders deutlich, dass gewisse pauschal oder final formulierte Urteile wie „Ontologie ist durch Ontologiekritik endgültig überwunden“, nicht nur überzogen, sondern auch sachlich falsch sind. Die Ontologiekritik gibt es nicht. Es gibt vielfältige, vielschichtige, in ihren Voraussetzungen reichhaltige, z.T. untereinander zerstrittene Positionen, welche die Ontologie ganz oder unter einer bestimmten Rücksicht kritisieren. Ontologinnen und Ontologen tun gut daran, dies ernst zu nehmen und die Bemühungen der Kritik so für ihr Fortkommen nutzbar zu machen. Allerdings können wir als Ergebnis auch festhalten, dass aus der Perspektive der Ontologie kein Anlass zur Resignation besteht. 


\section{Alltagsontologie}

Das am Ende des ersten Kapitels geschilderte Programm soll zunächst durch die Klärung des Begriffs einer Alltagsontologie auf den Weg gebracht werden (3.1). Alltagsontologie wird in der Folge mit den im zweiten Kapitel dargelegten Anliegen verschiedener Aspekte der Ontologiekritik, das sind Deflationierung, Naturalisierung, Simplifizierung, konfrontiert (3.2).

Wie kann man vom Standpunkt einer Alltagsontologie den konkreten Anfragen deflationistischer Kritik begegnen? Was kann man aber auch daraus lernen? Kann man nicht sogar bestimmte Ansätze aufgreifen und im Sinne von Alltagsontologie systematisch weiterentwickeln?

Der Umgang mit dem Naturalisierungsprogramm wird schwieriger, sprich kontroverser, zumal Vertreter von Naturalismus und Physikalismus ihre Theorien mitunter explizit als Gegenposition zu deskriptiver Ontologie, der ja Alltagsontologie verpflichtet ist, inszenieren. Hier ist Stellung zu beziehen, besonders unter der Rücksicht einer alltagsontologischen Klärung des Verhältnisses zwischen Naturwissenschaft und Ontologie.

Eine sich an alltäglicher Praxis orientierende Ontologie ist auf spezifische Weise Objekt der Kritik unter dem Vorzeichen des Simplifizierungsverdachts. Ist Alltagsontologie tatsächlich so naiv oder eben simpel, wie ihre Opponenten das behaupten? Diese Frage wird, unter Angabe von Gründen, $\mathrm{zu}$ verneinen sein. Auch hier wird allerdings das Anliegen im Mittelpunkt stehen, in der Diskussion mit kritischen Positionen das Profil unserer Alltagsontologie zu schärfen.

\subsection{Was bedeutet „Alltagsontologie“"?}

Die anvisierte Begriffsklärung soll in drei Schritten erfolgen. Zunächst wird auf das Gründungsdokument analytisch-deskriptiver Ontologie Bezug genommen, das ist Peter Strawsons Einzelding und logisches Subjekt (3.11). Alltagsontologie soll allerdings nicht schulmäßig aus Strawsons deskriptiver Ontologie oder Metaphysik, wie Strawson sagt, hergeleitet werden, sondern in systematischer Weiterentwicklung. Dazu sollen zunächst die Kriterien In- 
tuitivität, Entsprechung zu Grundstrukturen alltäglichen Sprechens bzw. Revidier- und Entwickelbarkeit als Merkmale einer Alltagsontologie dargestellt (3.12) und zu dem im ersten Kapitel entwickelten Ontologiebegriff in Bezug gesetzt werden (3.13).

\subsection{Peter Strawsons deskriptive Metaphysik}

Wenn eben von Einzelding und logisches Subjekt als von einem Gründungsdokument die Rede war, so kann im Hinblick auf eine Begriffsbestimmung von ,deskriptiver Metaphysik“ in besonderer Weise die Einführung zu diesem Werk hervorgehoben werden. ${ }^{154}$ Deshalb lohnt es sich, diese Textpassagen etwas genauer unter die Lupe zu nehmen.

Schon der Einstieg ist bemerkenswert und sei jedem bzw. jeder, der bzw. die sich in deskriptiver Metaphysik üben möchte, als Leitspruch ins Stammbuch geschrieben. So bekennt Strawson: „Metaphysik wurde vielfach revisionär, weniger oft deskriptiv betrieben. " Damit ist nicht nur das Gegenstück zu deskriptiver Theorienbildung in Metaphysik bzw. Ontologie angesprochen, nämlich die revisionäre, sondern auch der Hinweis, dass man sich mit der deskriptiven Zugangsweise in eine Minderheitenposition begibt. Das war zur Zeit der Abfassung von Strawsons Buch so und ist heute noch der Fall. Zum Glück ist die Zugehörigkeit zum Mainstream in der Ontologie kein absolutes Adäquatheitskriterium. Als deskriptive MetaphysikerIn muss man jedenfalls damit rechnen, „mit Misstrauen betrachtet“ $\mathrm{zu}$ werden und unter einem gewissen Auftrag zur Rechtfertigung zu stehen. Dies kann man freilich nicht nur als Last, sondern durchaus auch als Chance begreifen. Jedenfalls hat es noch keiner ontologischen Theorie geschadet, ihre Grundlagen zu reflektieren, mag das auch unter apologetischer Rücksicht geschehen.

Zum Kern der Sache gelangt man, wenn man folgende abgrenzende Charakterisierung beachtet: „Deskriptive Metaphysik begnügt sich damit, die tatsächliche Struktur unseres Denkens über die Welt zu beschreiben, revisionäre Metaphysik hat das Ziel, eine bessere Struktur hervorzubringen.“ Die Betonung in dieser Gegenüberstellung ist auf die Verben „,beschreiben“ versus

154 Strawson 1972, 9-13. Unter Anführungszeichen stehende Textbausteine in diesem Abschnitt 3.11 beziehen sich, wenn nicht anderes vermerkt ist, auf diese Seiten. 
„,hervorbringen“ zu legen. Revisionärer Metaphysik geht es darum, eine bessere oder adäquatere Struktur unseres Denkens zu entwickeln als jene, die sich im faktisch-alltäglichen Denken zeigt. Normalerweise geschieht das unter dem Verweis auf Wissenschaftlichkeit, wobei es in der Regel speziell naturwissenschaftliches Denken ist, welches dem gemeinen alltäglichen entgegengestellt wird. Ergänzt sei, dass dieses Hervorbringen oder Entwickeln letztlich kein Selbstzweck bleibt. Es zielt vielmehr darauf ab, die ,tatsächliche Struktur unseres Denkens“ auf bestimmte Weise zu erklären, etwa durch eine Ableitung aus den adäquateren oder besseren Strukturen, die dann auch relativ zu diesen tatsächlichen als die grundlegenden zu gelten haben. Dadurch wird auch die kognitive Relevanz bzw., im Kontext der Ontologie, die Weltdeutungskompetenz alltäglichen Denkens hergeleitet. Das macht den Gegensatz zu deskriptiver Metaphysik deutlich: Diese geht von der Überzeugung aus, dass es bzgl. des faktischen, alltäglichen Denkens schlicht nichts zu erklären gibt. Es ist, so wie es ist, relevantes Denken über die Welt. Somit genügt es, dieses zu beschreiben.

Im Hinblick auf die zitierte Stelle könnte man sich natürlich die Frage stellen, was „,die Struktur unseres Denkens“ mit der Struktur der Wirklichkeit zu tun hat, mit ,der Welt“, um die es ja in der Metaphysik bzw. in der Ontologie geht. Haben wir es hier mit dem Versuch einer Rückführung der Ontologie auf Erkenntnistheorie zu tun, gar mit der Reduzierung des Seins der Wirklichkeit auf das Denken über die Welt, also mit blankem Idealismus? Ohne jeden Verdacht in diese Richtung vorschnell vom Tisch wischen zu wollen, tun wir im Sinne einer angebracht wohlwollenden Interpretation gut daran, eine wichtige Prämisse, die bei Strawson wohl mitgemeint ist, explizit zu machen: Ontologische Strukturen und denkerische Strukturen haben, vorsichtig gesagt, miteinander zu tun. Beziehungsweise eine ontologische Theorie über die Grundstrukturen der Welt ist, unabhängig bzw. losgelöst von Überlegungen, wie wir (über die Welt) denken bzw. sprechen, eine problematische Konstruktion. Selbst wenn man dieser Prämisse reserviert gegenübersteht, bleibt zu beachten, dass die von Strawson gemeinte Strukturanalogie zwischen Denken und Wirklichkeit nicht nur die Charakterisierung deskriptiver Metaphysik betrifft, sondern insgesamt die Metaphysik. Sie kann 
also nicht ausschließlich gegen das Anliegen deskriptiver Metaphysik vorgebracht werden.

Ein weiteres wesentliches, sprich definierendes Merkmal deskriptiver Metaphysik besteht darin, dass sie durch den ,,unbegrenzten Umfang und die Allgemeinheit der Fragestellung" charakterisiert ist. Demgegenüber bietet revisionäre Metaphysik eine ,enger begrenzte und partielle begriffliche Untersuchung“ bzw. geht von einer solchen aus. Darin ist, wie Strawson einräumt, zunächst ein Startvorteil der revisionären Metaphysik auszumachen. Geht man von Ergebnissen methodisch begrenzter und somit partieller Zugangsweisen zu Forschungsobjekten aus, wie sie z.B. Naturwissenschaften liefern, kann man dafür eine gewisse, sprich dazu relative „Selbstverständlichkeit““ beanspruchen, die nicht zuletzt auch vom Erfolg besagter Zugangsweise in der Erklärung und Prognostizierung von Weltverläufen profitiert. In diesem Sinne kann revisionäre Metaphysik „Prägnanz“ und „Intensivität“, wie Strawson sagt, beanspruchen, gerade aufgrund der besagten Partialität. Deskriptive Metaphysik hat aufgrund ihrer Allgemeinheit und Universalität diesen sicheren Ausgangshafen in der Theorienbildung nicht aufzuweisen. Im Gegenzug aber erzielt sie Autonomie gegenüber einzelwissenschaftlicher Vorgangsweise, durchaus auch mit dem Anspruch, das in Einzelwissenschaften enthaltene Partielle und Begrenzte in einen universalen Rahmen zu integrieren und nach Maßgabe eigener Methodik systematisch zu erfassen. Darüber hinaus ist sie nicht auf die aus Einzelwissenschaften übernommene, also relative Selbstverständlichkeit angewiesen, die ja im Kontext philosophischer Argumentation doch wieder zu hinterfragen ist. Für die deskriptive Metaphysik gilt, dass sie „,keiner weiteren Rechtfertigung bedarf als der der Forschung im Allgemeinen“. Das könnte man im Sinne der traditionellen Bestimmung der Metaphysik verstehen, dass sich diese als Prinzipienwissenschaft ausschließlich selbst begründen kann und Selbstverständlichkeiten von außen weder aufnehmen muss noch darf.

Eine weitere wesentliche Charakterisierung von deskriptiver Metaphysik geht von Strawsons Feststellung aus, dass sich Begriffe „tatsächlich ändern“, „,und das nicht nur - wenn auch überwiegend - an der Peripherie des Spezialforschers“. Diese Änderungen aber ,wirken auf das normale Denken zurück“. Diese Bemerkungen können wir nicht nur wissenschaftstheoretisch als Hin- 
weis auf die Fortentwicklung einzelwissenschaftlicher Spezialsprachen verstehen. Es geht durchaus auch um das sprachwissenschaftliche Faktum, dass natürliche Sprachen keine starren Gebilde sind, sondern in permanenter Dynamik bestehen. Beides kann man nicht losgelöst betrachten von dem, was die deskriptive Metaphysik als die tatsächliche Struktur unseres Denkens beschreibt. Obwohl es ein Fehler wäre, die „Metaphysik nur auf diese historische Weise zu sehen“, ${ }^{155}$ kann man somit auch keine reine, dogmatische Statik unserer faktischen Denkstrukturen annehmen. Der von Strawson anvisierte Mittelweg zwischen historisierender und dogmatisch starrer Metaphysik besteht darin, zunächst anzunehmen: „Es gibt faktisch Kategorien und Begriffe, die sich in ihrem Grundcharakter überhaupt nicht ändern." Allerdings schließt das nicht aus, dass es theoretisch Elemente der Grundstrukturen unseres Denkens geben mag, die sich weiterentwickeln, wenn schon nicht in ihrem „Grundcharakter“, so doch in manchen ihrer bestimmenden Merkmale. Wir haben es also in der deskriptiven Metaphysik nicht, wie manche InterpretInnen behaupten (siehe u.a. 2.33, wo von Susan Haack die Rede ist), mit einem Produkt ungerechtfertigt ahistorischer Statik zu tun, sondern mit einer Theorie, die charakteristische Entwickel-, ja auch Revidierbarkeit aufweist.

$\mathrm{Zu}$ ergänzen ist, dass sowohl die theoretische Varianz als auch die Annahme eines faktisch vorhandenen, stabilen Grundbestands der Strukturen tatsächlichen oder alltäglichen Denkens nicht nur historisch, sondern durchaus auch geographisch, oder besser kulturübergreifend verstanden werden können: Faktisch zeichnet sich die tatsächliche Denkstruktur von Menschen auch noch so unterschiedlicher Kulturen durch einen gemeinsamen Bestand eines Zentralbereichs aus, ohne dass man von vornherein eine starre Übereinstimmung in allen Merkmalen behaupten muss. Kulturvarianz, so verstanden, gehört ebenfalls zu den Merkmalen deskriptiver Metaphysik.

Ein weiterer Punkt: Zur Bewertung der Distinktion zwischen deskriptiver und revisionärer Metaphysik gibt Strawson zu bedenken, dass in konkreten Positionierungen bei „keinem wirklichen Metaphysiker“ eine Reinkultur des einen wie des anderen gefunden werden kann. Das ist bemerkenswert, wenn man bedenkt, wie kontrovers die Gegenüberstellung mitunter aufgebaut wird. Strawsons Relativierung dieser Unterscheidung können wir wohl zunächst so

155

Hervorhebung Kanzian 
verstehen, dass sich auch revisionäre Metaphysik faktisch der tatsächlichen Grundstrukturen des Denkens bedienen muss. Faktisches Denken gibt nämlich die Rahmenbedingungen einzelwissenschaftlicher Theorienbildungen $a b$, was uns in folgenden Abschnitten noch eingehender beschäftigen wird. Einzelwissenschaftliche Theorienbildung aber bildet das Motiv zu und den Ausgang von revisionär-metaphysischen Überlegungen. Allerdings ist es auch so, dass wir die Grundstrukturen unseres alltäglichen Denkens ,nicht an der Oberfläche der Sprache erkennen“ können. Deskriptive Metaphysik hat sich deshalb bei ihrer Beschreibung des faktischen, alltäglichen Denkens auch Analyseelementen zu bedienen, welche der Tiefenstruktur unserer Sprache gerecht werden. Diese Analyseelemente aber können nicht selbst wieder aus dem Alltagsdenken entlehnt werden. Dazu sind sie zu speziell, mitunter wohl auch zu technisch, und erfordern deshalb auf der Reflexions- oder Metaebene an manchen Stellen eine Revision allzu einfacher, ja naiver Verständnisweisen des faktisch-alltäglichen Sprechens.

Diese bedingte Relativierung der Abgrenzung zwischen deskriptiver und revisionärer Metaphysik steht bei Strawson allerdings nicht in Gegensatz zu einer klaren Bekundung des grundlegenden Status der Ersteren. So schreibt er unmissverständlich: „Revisionäre Metaphysik ist der deskriptiven Metaphysik verpflichtet." Diese Behauptung bringt es auf den Punkt, dass für ihn die Autonomie, die Voraussetzungslosigkeit, sowie der integrative Charakter der deskriptiven Metaphysik, schließlich der zuletzt angeführte grundlegende Charakter des Faktisch-Alltäglichen speziell für einzelwissenschaftliches Denken, eine klare Priorisierung bedingt. Dem entspricht die Einstellung, dass es ,die Selbstverständlichkeiten des am wenigsten entwickelten Denkens [sind], die dennoch unabweislich den Kern für das begriffliche Rüstzeug auch des anspruchvollsten Kopfes ausmachen“.

In diesem Sinne geht es in der Metaphysik bzw. in der Ontologie darum,

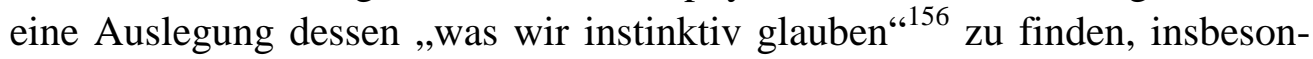
dere natürlich bezüglich dessen, was existiert. Dass dies bei Strawson nicht als Plädoyer für eine naive Umlegung alltäglicher Redeweisen auf eine Theorie der Grundstrukturen der Wirklichkeit gemeint ist, sollte aus den vorhergehenden Ausführungen anfänglich deutlich geworden sein, und durch

${ }^{156}$ Strawson 1972, 317. 
den Versuch einer Weiterentwicklung seines Ansatzes im Sinne einer Alltagsontologie, die auch den Einwänden der aktuellen Metaontologie gewachsen ist, noch klarer werden.

\subsection{Grundkriterien für Alltagsontologie}

Im Sinne der einleitenden Bemerkungen zu diesem Abschnitt sollen nun Intuitivität, Entsprechung zu Grundstrukturen unseres alltäglichen Sprechens bzw. Revidier- und Entwickelbarkeit als charakterisierende Merkmale einer Alltagsontologie vorgestellt werden. ${ }^{157}$ Dabei wird sich zeigen, dass diese Merkmale aus einer systematischen Weiterentwicklung bestimmter Grundelemente von Strawsons deskriptiver Metaphysik zu verstehen sind.

Beginnen wir bei Intuitivität. Es handelt sich dabei um einen Topos, der im ontologischen Diskurs durchaus geläufig ist; für gewöhnlich freilich als polemisches Attribut. Der Verweis auf Gegen- oder Kontraintuitivität (engl.: counterintuitivity) wird nämlich gar nicht so selten, quasi als Argument, gegen eine These ins Treffen geführt, wenn die Verständlichkeit oder Nachvollziehbarkeit dieser These in Zweifel gezogen werden oder einfach dem Gegner eine gewisse Beweislast aufgebürdet werden soll. Hier wird das Postulat der Intuitivität allerdings zunächst von Strawson her eingeführt. Insofern nämlich, als deskriptive Metaphysik, wie gegen Ende von 3.11 vermerkt, von dem auszugehen versucht, „was wir instinktiv“ glauben, bzw. bei den „Selbstverständlichkeiten des am wenigsten entwickelten Denkens“ ansetzt. Der Kontraintuitivitätshinweis würde demnach zunächst besagen, dass die adressierte Position das nicht tut. Sie ist keine deskriptiv metaphysische.

Ohne dies an dieser Stelle als Argumentationsstrategie bewerten zu wollen, können wir einen Schritt weitergehen und fragen, wie genau jene ,instinktiven" oder eben intuitiven Einstellungen zu verstehen sind, die dabei ins Spiel gebracht werden. Eine Liste möglicher Merkmale hat jedenfalls eine gewisse Spontaneität zu beinhalten, wobei „spontan“ hier weder als vorschnell noch als naiv zu verstehen ist, sondern als Gegensatz zu wissenschaft-

${ }^{157}$ Dabei orientiere ich mich an Überlegungen in den Monographien „Ding - Substanz - Person“ bzw. „Wie Dinge sind“: Kanzian 2009, Abschnitt I - 1: Was ist eine Alltagsontologie, bzw. Kanzian 2016, Abschnitt I - 2: Deskriptive Ontologie. 
lich reflektierten Einstellungen. Vielleicht ist auch das Attribut „,vorwissenschaftlich“ hilfreich. Das Objekt der gemeinten Einstellungen ist, um einen zweiten Punkt anzusprechen, jedenfalls ein propositionaler Gehalt. Man ist auf einen Inhalt bezogen, der darin besteht, dass etwas der Fall ist bzw. so oder so der Fall ist. Dass mein Auto heute noch dasselbe ist wie zu jenem Zeitpunkt, zu dem ich es gekauft habe, wäre ein solcher Inhalt. Das bedingt auch, dass man sich zu diesen Gehalten entweder zustimmend oder ablehnend verhalten kann. Sich spontan neutral dazu zu verhalten, ob etwas der Fall ist, wird damit ausgeschlossen. Natürlich kann man sich einen Moment der Unsicherheit bzgl. eines Gehalts vorstellen. Das aber ist dann eben keine intuitive Einstellung zu etwas, sondern deren Fehlen. Freilich ist, drittens, nicht jede Intuition, sprich spontane Einstellung, ontologisch relevant. Das sind nur solche, deren Gehalt in allgemeinen und universalen Gegebenheiten besteht, nicht in konkret Einzelnem und Besonderem. Wenn jemand beispielsweise ohne lange Spekulation zur Auffassung gelangt, dass seinem Auto wieder einmal die Durchfahrt einer Waschstraße guttäte, wird das für eine Alltagsontologie irrelevant bleiben. Nicht jedoch die spontan positive Einstellung, dass sich Dinge wie Autos durch ihre Selbigkeit durch die Zeit auszeichnen. Von ontologischer Relevanz ausgeschlossen bleiben auch solche spontanen Einstellungen, die in hohem Maße subjektiv sind; fernab davon, so weit von einer Vielzahl von Menschen geteilt zu werden, dass das deren soziales $\mathrm{Zu}$ sammenleben befördert. Intuitionen bzgl. des Bestehens von Identität durch die Zeit von Autos sind nicht subjektiv in diesem Sinn. Sie werden, von möglichen wenigen Ausnahmefällen abgesehen, spontan von Menschen geteilt. Zum Glück, könnte man sagen, zumal geklärte Eigentumsverhältnisse wesentlich für gedeihliche gesellschaftliche Kooperation sind. Intuitive Einstellungen oder kurz Intuitionen sind, um es zusammenzufassen, spontane, positive oder negative Einstellungen zu bestimmten Inhalten, die, im Fall ontologischer Relevanz, auf allgemeine und universale Gegebenheiten bezogen sind und intersubjektiv geteilt werden.

Wie können wir das nun als ein Adäquatheitskriterium für eine ontologische Theorie formulieren? Jedenfalls nicht so, dass man damit einer einfachen oder naiven Umlegung von Alltagseinstellungen in eine Ontologie das Wort redete. Zudem sei auch das „ein“ in ,ein Adäquatheitskriterium“ unter- 
strichen. Das besagt, dass es noch andere, allgemeinere Kriterien geben mag, wie innere Konsistenz, Ökonomie etc., die auch im Kontext von Alltagsontologie maßgeblich für die Beurteilung von Thesen sind. Dann aber kann durchaus davon die Rede sein, dass eine These im Sinne einer Alltagsontologie als adäquat zu erachten ist, wenn sie Teil eines Rahmens zur Erläuterung dessen abgibt, was wir spontan zu bestimmten Gehalten mit ontologischer Relevanz meinen. Eine These, die z.B. die Annahme der diachronen Selbigkeit unserer Autos ontologisch rechtfertigt, kann in diesem Sinn als alltagsontologisch adäquat erachtet werden. Alltagsontologische Adäquatheit kann natürlich an dieser Stelle nur als ein relatives Kriterium gelten. Das sei gesagt, um die einleitenden Überlegungen zum Intuitivitätskriterium als ontologisches Argument aufzugreifen. Wenn aber, wofür zusätzlich Gründe angeführt werden müssen, Alltagsontologie als ontologisches Projekt Erfolg hat, dann ist eine These als ontologische gerechtfertigt, wenn sie alltagsontologisch adäquat ist. Viel Arbeit liegt jedenfalls vor uns.

In diesem Sinn können wir zur Darlegung eines weiteren besonderen Kriteriums für Alltagsontologie übergehen, nämlich die Entsprechung zu Grundstrukturen unseres alltäglichen Sprechens; wobei hier Strawsons Überzeugung, dass sich "gewöhnliches Denken" in diesen basalen sprachlichen Strukturen manifestiert, angenommen werden soll. Unter einer „Grundstruktur" sei jedenfalls eine Struktur verstanden, die relativ zu verschiedenen natürlichen Sprachen invariant ist, einzelne Sprachen als Ganze betrifft und mit Mitteln der Grammatik dieser einzelnen Sprachen nicht expliziert werden kann, weil sie dieser Grammatik selbst zugrunde liegt.

Die Subjekt-Prädikat-Struktur habe ich an anderer Stelle als eine solche Grundstruktur versucht darzustellen. ${ }^{158}$ Dass sich dies auch im Anschluss an Strawson nahelegt, ergibt sich schon daraus, dass der gesamte zweite Hauptteil von Einzelding und logisches Subjekt einer Interpretation dieser Struktur gewidmet ist. Hier müssen wir nicht die ausführlichen Darlegungen Strawsons im Detail berücksichtigen. Es reicht der Hinweis, dass es wesentlich für unser gewöhnliches Denken ist, sich in der sprachlichen Struktur von Subjekt und Prädikat auszudrücken: „Die Tafel ist grün“ bzw. „Hans sitzt neben

${ }^{158}$ Siehe vorhergehende Fußnote 
Luise" sind einfache Beispiele, die auch gleich zeigen, dass Prädikate unterschiedliche grammatikalische Gestalt, etwa Ein- bzw. Mehrstelligkeit, annehmen können.

Vollständigkeitsbeweis, dass diese Struktur alle natürlichen Sprachen prägt, müssen wir hier keinen erbringen, gerade unter Verweis auf die Kulturvarianz, die theoretisch auch eine Abwandlung von Grundstrukturen bedingen kann. Auch muss man in Betracht ziehen, dass sich die Subjekt-Prädikat-Struktur in verschiedenen Sprachen auf unterschiedliche Weise grammatikalisch manifestiert. Jedenfalls können wir davon ausgehen, dass eine für unsere Zwecke hinreichende Invarianz vorliegt und der grundlegende Charakter dieser Struktur für grammatikalische Oberflächen von Sprachen ebenso gegeben ist wie das Faktum, dass nicht nur Teile von Sprachen, sondern stets Sprachen als Ganze nach Subjekt und Prädikat strukturiert sind. ${ }^{159}$

Eine weitere Grundstruktur in eingeführtem Sinne besteht darin, dass natürliche Sprachen mit einem bestimmten Apparat der Identifikation von Gegenständen ausgestattet sind, die eine enge Verbindung mit der bezugnehmenden oder referierenden Funktion aufweist. Ebenso zeichnet natürliche Sprachen aus, dass man bei oder in ihnen bestimmte Abhängigkeiten in der Identifikation unterschiedlicher Objekte feststellen kann. Damit beschäftigt sich Strawson im ersten Teil von Einzelding und logisches Subjekt: ${ }^{160}$ Einzeldinge oder Individuen sind jene Objekte, auf deren Identifikation das sprachlich artikulierte alltägliche Denken ausgerichtet ist. Unter den Individuen sind es materielle Körper, deren Identifikation grundlegend, die der Ereignisse z.B. aber abhängig ist, und zwar von der Identifikation ihrer „Träger“, das sind eben materielle Körper. Für Strawson ist klar, dass wir „für gewöhnlich“ auf Ereignisse, etwa Spaziergänge, identifizierend nur Bezug nehmen können, wenn wir auf etwas oder jemand Bezug nehmen, der eben spazieren

${ }^{159}$ Das schließt nicht aus, dass es satzartige sprachliche Ausdrücke geben mag, bei denen diese Struktur nicht augenfällig ist, z.B. einfache Imperative wie „Geh!“. Diese aber sind nur verständlich als grammatikalisch reglementierte Abkürzungen von Sätzen, welche der Struktur entsprechen, etwa „Du sollst jetzt gehen!“. Darin zeigt sich auch, dass die SubjektPrädikat-Struktur nicht an der Oberfläche der Grammatik liegt, sondern eben für diese grundlegend ist. Dass die Struktur „Sprachen als ganze“ betrifft, meint nun, dass es keinen Bereich in ihnen gibt, der nicht auf diese oder analoge Weise rekonstruiert werden könnte.

${ }^{160}$ Grundlegend: Strawson 1972, $17 \mathrm{ff}$. 
geht. Dieses Etwas oder dieser Jemand aber gehört der Kategorie der materiellen Körper an. ${ }^{161}$ Besagtes Phänomen ist sprachinvariant, für die grammatikalische Ausprägung von Sprachen grundlegend und lässt sich nicht auf einen Teilbereich von Sprachen beschränken.

In Entsprechung zum oben bzgl. Intuitionen Gesagten, können wir auch unter Verweis auf diese Grundstrukturen alltäglichen Sprechens ein Adäquatheitskriterium für Alltagsontologie formulieren. Hier ist allerdings besonderer Wert darauf zu legen, dass damit keinesfalls eine einfache oder naive Umlegung sprachlicher Strukturen auf eine ontologische Theorie gemeint sein kann. Das wäre Simplifizierung im abgehandelten Sinn (siehe Abschnitt 2.32). Dennoch können wir festhalten, dass eine These im Sinne einer Alltagsontologie als adäquat zu erachten ist, wenn sie Teil eines Rahmens zur Erläuterung der Subjekt-Prädikat-Struktur bzw. des identifizierenden Apparats unserer Alltagssprache ist, unter Berücksichtigung von einschlägigen Abhängigkeiten bzgl. der Identifikation verschiedener Arten von Objekten, etwa jener der Ereignisse von derjenigen der materiellen Körper oder Dinge. Wenn etwa eine Ontologie Dinge kennt, wie den Spaziergänger im Beispiel, und Ereignisse, z.B. sein Spazierengehen, wobei Letztere in spezifischer Weise von Ersteren abhängen, können wir eine solche Erläuterungskompetenz annehmen. Das gilt ebenso für eine Ontologie mit Entitäten wie Tafeln, Hans und Luise, die als Träger von Eigenschaften fungieren können, welche sie charakterisieren und sie untereinander in Beziehung setzen. Natürlich gilt auch von dieser Stelle aus die vorläufige Relativität dieses Adäquatheitskriteriums als ontologisches Argument. Durch Verweis auf dieses Kriterium ist eine Theorie erst dann zu widerlegen, wenn der Vorzug einer Alltagsontologie über ihre Konkurrenz, wir können sie weiter „,revisionäre Ontologie“ nennen, erwiesen ist.

Damit können wir zu einem weiteren Gesichtspunkt kommen, der es uns gestattet, Alltagsontologie zu charakterisieren, nämlich deren Entwickel- bzw. Revidierbarkeit. Dieser dritte Gesichtspunkt lässt sich im Kontext einer Bestimmung von Alltagsontologie nicht isoliert von den ersten beiden, Intuitivi-

${ }^{161}$ Dies ist im Hinblick auf Ereignisse pointiert formuliert das Ergebnis von Strawson 1972, Teil I, Abschnitt 3: Grundlegende Einzeldinge. 
tät und Entsprechung zu den Grundstrukturen alltäglichen Sprechens, verstehen. Intuitionen, auch ontologisch relevante, können sich weiterentwickeln, sich damit im Hinblick auf bestimmte Inhalte hinsichtlich Zustimmung bzw. Ablehnung ändern. Ebenso gilt, dass Sprachen keine statischen Gebilde sind, sondern einer Entwicklungsdynamik unterliegen, von der Elemente ihrer Grundstrukturen nicht ausgenommen werden können. Wenn dem so ist, ergibt sich dieses dritte Charakteristikum von Alltagsontologie aus den vorhin angenommenen Kriterien.

In Zusammenschau mit den Ausführungen von Abschnitt 3.11 lässt sich auch an Entwickel- bzw. Revidierbarkeit eindeutig Strawson-Bezug festmachen, unter besonderer Berücksichtigung der Evolution natürlicher Sprachen, die ja, so Strawson, zumindest theoretisch, nicht nur deren „Peripherie“ betrifft, sondern auch deren Kernbereich.

Wie wir das bei Strawsons deskriptiver Ontologie gemacht haben, können wir auch im Hinblick auf Alltagsontologie bei diesem ,nicht-statischen“ oder „nicht-dogmatischen“ Aspekt eine synchrone und eine diachrone Dimension unterscheiden. Während mit „diachron“ die historische Entwickelbarkeit von Grundzügen natürlicher Sprachen gemeint ist, sowie die Änderung von ontologisch relevanten Intuitionen durch die Zeit, ist mit „synchron“ die Kontextbzw. Kulturvariabilität von Alltagsontologie gemeint. Wir können faktisch einen Kern von Intuitionen zu Grundstrukturen der Wirklichkeit annehmen, der von Menschen sämtlicher Kulturen geteilt wird bzw. eine weitgehende Invarianz sprachlicher Grundstrukturen in natürlichen Sprachen. Dennoch ist es prinzipiell denkbar, dass es Menschengruppen gibt, in denen von unserer Kultur vollkommen verschiedene, ontologisch relevante, intuitive Einstellungen vorherrschen. Ebenso sind, wie erwähnt, auch Kulturen mit alternativen sprachlichen Grundzügen denkbar.

Als ein weiteres Kriterium für Alltagsontologie können wir die Entwickel- bzw. Revidierbarkeit ihrer Thesen, sowohl im historischen als auch im kulturvergleichenden Sinn, annehmen. Betont sei im Sinne des eben Dargelegten, dass hier jene Entwickel- und Revidierbarkeit gemeint ist, die sich im Zusammenhang mit den beiden anderen Kriterien für Alltagsontologie ergibt, nämlich Intuitivität und Entsprechung zu den Grundstrukturen alltäglichen Sprechens, in welchen sich „,normales Denken“ manifestiert. 
Zusammenfassend können wir somit als Grundkriterien einer Alltagsontologie annehmen, dass sie bei ihrer Theorienbildung ontologisch relevante intuitive Einstellungen (in eingeführtem Sinn) berücksichtigt und eine Erläuterung von Grundstrukturen alltäglichen Sprechens, in denen sich unser „gewöhnliches Denken" manifestiert, anzielt. Aufgrund der Entwickel- bzw. Revidierbarkeit beider gilt für Alltagsontologie, dass sie grundsätzlich offen ist für Modifikationen und somit keinen Anspruch auf eine definitive Gestalt erheben kann.

Darin unterscheidet sie sich von ihrem, mit Strawson als „revisionär“ zu bezeichnenden Gegenstück. Für revisionäre Ontologie ist Kontraintuitivität nicht nur hinzunehmen, sondern gegebenenfalls sogar anzustreben, wenn sich, etwa ausgehend von Ergebnissen naturwissenschaftlicher Forschung, eine Abweichung unseres „normalen Verstehens“ der Grundstrukturen der Wirklichkeit nahelegt. Wissenschaft revidiert alltägliches Verstehen der Welt. ${ }^{162}$ Desgleichen spielen die angenommenen Grundstrukturen alltäglichen Sprechens, die Subjekt-Prädikat-Struktur bzw. Identifikation mit Identifikationsabhängigkeiten, keine Rolle. Das Gegenteil ist der Fall: Die Gliederung in Subjekt und Prädikat ist, wenn schon nicht aufzuheben, so in einer revidierten oder ,idealen“ Sprache zumindest zu relativieren. ${ }^{163}$ Identifikationsabhängigkeiten zwischen Objekten unterschiedlicher Kategorien verlieren schon deshalb an Bedeutung, weil revisionäre Ontologien in der Regel auf mehrkategoriale Ordnungsschemata verzichten.

Entwicklung und Modifikation spielt in revisionären Ontologien durchaus eine Rolle. Allerdings werden diese in ihrem Kontext nicht durch den Bezug auf die Abänderung intuitiver Einstellungen bzw. die Dynamik natürlicher Sprachen begründet, sondern durch den Fortschritt der von ihr akzeptierten

\footnotetext{
162 Quine 1994, 93 bringt diese revisionäre Einstellung auf den Punkt, indem er bekundet: „Unnaturalness in philosophy is all right“.

${ }^{163}$ Auch diesen Gedanken kennen wir von Quine (u.a. 1948) her, der in seiner „,kanonischen“ Analyse von Sätzen der Alltagssprache (vermeintliche) Subjekte als Prädikate interpretiert, v.a. um negative Existenzaussagen widerspruchsfrei formulieren $\mathrm{zu}$ können. So wird aus dem Subjekt in „Pegasus existiert nicht“ in der Sprache der Prädikatenlogik ein Prädikat: „Es gibt nicht etwas, das pegasiert." Eine noch radikalere revisionäre Negierung der Subjekt-Prädikat-Struktur hat übrigens Lorenz Puntel geboten, u.a. in ders. 2006, $196 f$.
} 
Autoritäten in Sachen Wirklichkeit, normalerweise sind dies die Naturwissenschaften, allen voran die Physik.

Möchte man tatsächlich Alltagsontologie betreiben, muss man nun, wie auch Strawson das getan hat, eine Bewertung vornehmen. Strawson spricht ja vom grundlegenden Charakter der deskriptiven Metaphysik gegenüber der revisionären; sogar davon, dass revisionäre Metaphysik der deskriptiven verpflichtet ist. Seine Argumentation für diesen Standpunkt muss hier nicht wiederholt werden. Allerdings ist das damit zum Ausdruck gebrachte Anliegen zu unterstreichen. So muss es in der Alltagsontologie darum gehen, Gründe vorzubringen, warum eine - kurz gesagt - sich am alltäglichen Verstehen der Wirklichkeit orientierende Ontologie gegenüber einer „,wissenschaftlichen“, sprich naturwissenschaftlich ausgerichteten, der Vorzug zu geben ist. Erst dann können die Kriterien für eine Alltagsontologie als nicht-bedingte bzw. nicht-relative Adäquatheitskriterien einer ontologischen Weltbeschreibung gelten. Wenn das gelingt, kann allerdings die Entsprechung zu intuitiven Einstellungen, die Kompetenz in der Interpretation von Grundstrukturen alltäglichen Sprechens, letztlich auch spezifische Entwickel- und Modifizierbarkeit als Argument für eine ontologische Theorie dienen; Kontraintuitivität, Ignoranz der faktischen sprachlichen Grundstrukturen, damit verbundenes Missverstehen der Modifizierbarkeit ontologischer Theorien, bzw. deren Negierung, allerdings als Gründe, ontologische Theorien zurückzuweisen.

Mit diesem Ausblick können wir zum dritten, in der Hinführung zu 3.1 genannten Schritt zur Entwicklung des Begriffs einer Alltagsontologie kommen und die nach Strawson angenommenen Grundkriterien zu dem im ersten Kapitel dargelegten Ontologiebegriff in Bezug setzen.

\subsection{Ontologie als Alltagsontologie}

Um das eben Gesagte anzugehen, soll zunächst der angesprochene Ontologiebegriff des ersten Kapitels aufgegriffen werden: Die Ontologie, so wurde dort ausgeführt, ist eine systematische Reflexion auf jene Voraussetzungen bzgl. dessen, was ist oder existiert, wie sie in verschiedenen Praktiken gemacht werden; mit dem Ziel, eine konsistente Rahmentheorie über die Klärung dieser Voraussetzungen zu erlangen. „Systematisch“ bedeutet in diesem Zusammenhang, wohlgemerkt ohne Ausschluss von Alternativen, zunächst 
analytisch-philosophisch vorzugehen. In Anwendung der begrifflichen Werkzeuge, aber auch unter Berücksichtigung einschlägiger Themen der analytischen Standarddiskussion, soll argumentativ nachvollziehbar über die Voraussetzungen bzgl. Existierendem nachgedacht werden. Mit „Rahmentheorie" ist, um auch diesen Gesichtspunkt in Erinnerung zu rufen, der universale, allgemeine und integrative Charakter des anvisierten Entwurfs einer Ontologie gemeint. „Universal“ besagt dabei zunächst, dass keine Praxis von einer einschlägigen Untersuchung ausgeschlossen werden darf. Im Hintergrund steht die Überzeugung, dass nicht nur die Praxis in diversen Wissenschaften hinsichtlich ihrer Voraussetzungen bzgl. dessen, was es gibt, für die Ontologie relevant ist, sondern auch die alltägliche Praxis in der Vielfalt ihrer Erscheinungsformen. Natürlich ist nicht alles, was wir denken, sprechen, meinen, hoffen, auf Grundelemente der Wirklichkeit bezogen. Der Punkt ist vielmehr, dass zu den Voraussetzungen dieser Praktiken, seien sie dem Objekt nach noch so banal, bestimmte, in den allermeisten Fällen implizite Annahmen darüber gehören, was es gibt; bzw. wie das, was man als existierend annimmt, existiert, insofern es existiert. Welches Objekt unser Handeln auch immer hat, ohne ontologische Voraussetzungen kann es nicht vollzogen werden. Das Geschäft unserer Disziplin ist es, das explizit zu machen, in nachvollziehbarer Art, systematisch eben. Mit dem Bezug auf Annahmen bzgl. dessen, was existiert ,insofern es existiert“ ist auch schon die Eigenart einer ontologischen Rahmentheorie bzgl. Existierendem umrissen. Die Ontologie visiert eine allgemeine Rahmentheorie bzgl. Existenzvoraussetzungen diverser Praktiken an, sprich eine Theorie, die nicht von beschränkten methodischen Zugangsweisen bzw. Interessen geprägt ist. Die Ontologie reflektiert natürlich auch auf einzelwissenschaftliche Praktiken. Allerdings, um es wiederholend zu unterstreichen, auf deren Existenzvoraussetzung und, auf das kommt es hier an, in einer methodisch nicht an den Einzelwissenschaften orientierten Weise. „Integrativ“, um noch das dritte Charakteristikum ontologischer Theorien anzusprechen, macht noch einmal die Perspektive ontologischer Reflexion auf die ihr eigentümlichen Gegenstände deutlich: Existenzvoraussetzungen bestimmter Praktiken sollen in einen Zusammenhang gebracht werden, untereinander und in Bezug auf eine Gesamttheorie bzgl. Existierenden, welche dann eben als Rahmentheorie fungiert. 
Wie kann man nun diesen Ontologiebegriff in Beziehung setzen zu jenen Kriterien, die im letzten Abschnitt als maßgeblich für Alltagsontologie vorgestellt wurden: Intuitivität, Entsprechung zu den Grundstrukturen alltäglichen Sprechens, schließlich Entwickel- und Revidierbarkeit?

Bei der Beantwortung dieser Frage können wir dabei ansetzen, dass bislang von jenen Praktiken, deren Voraussetzungen im Hinblick auf Existenz zu reflektieren sind, ohne Einschränkung und auflistend die Rede gewesen ist. Für die Ontologie als Universaltheorie ist es ja nur maßgeblich, alle Praktiken in Betracht zu ziehen, um diese dann nach Maßgabe der ihr eigenen Allgemeinheit zu reflektieren und deren Existenzvoraussetzungen systematisch in Bezug zueinander zu setzen.

Nun können wir, in einem nächsten Schritt, aber dazu übergehen, eine bestimmte Praxis, besser vielleicht ein bestimmtes Praxisfeld, genauer unter die Lupe zu nehmen. Im Hinblick auf die Entwicklung von Alltagsontologie wird das, wenig überraschend, die alltägliche Praxis sein. Dabei, sprich bei der Reflexion auf die Existenzvoraussetzungen alltäglicher Praxis, zeigt sich aber, dass die drei im vorhergehenden Absatz diskutierten Kriterien eine maßgebliche Rolle spielen. Bei der Interpretation der Existenzvoraussetzungen alltäglicher Praxis spielen nämlich Intuitionen oder, wie Strawson sagt, das, was wir „,instinktiv“ annehmen, eine maßgebliche Rolle, und zwar als ein Adäquatheitskriterium in eingeführtem Sinn. Es ist jene Interpretation der Existenzvoraussetzungen alltäglicher Praxis zu bevorzugen, welche mit spontanen, vorwissenschaftlichen Einstellungen bzgl. dessen, was existiert, zurechtkommt, gegenüber einer, welche ontologisch relevanten Intuitionen entgegenläuft. Nehmen wir an, um an ein Beispiel aus dem vorhergehenden Abschnitt anzuknüpfen, wir akzeptieren es intuitiv, dass sowohl die Gebrauchsgegenstände unserer Alltagswelt, als auch deren belebte BewohnerInnen, in einem strikten Sinn nicht nur zu einem Zeitpunkt, sondern auch durch die Zeit mit sich identisch sind, so ist jener Interpretation der Existenzvoraussetzungen unserer alläglichen Praxis der Vorrang zu geben, die auf solchen endurer aufbaut; gegenüber einer Deutung, welche dieselben negiert.

Analoges lässt sich bzgl. des zweiten Kriteriums anführen: Als eine authentische Auslegung der Voraussetzungen bzgl. Existierenden alltäglicher Praxis gelten Thesen, welche den Grundstrukturen alltäglichen Sprechens 
entsprechen. Das ergibt sich schon daraus, dass alltägliches Sprechen ja ein paradigmatisches Beispiel alltäglicher Praxis ist. Dies kann im Hinblick auf die genannten Elemente alltäglicher Sprechpraxis, Subjekt-Prädikat-Struktur bzw. Identifikation bzw. Identifikationsabhängigkeiten, konkretisiert werden. Somit kann eine Theorie dann als adäquate Auslegung der Existenzvoraussetzungen alltäglicher Praxis erachtet werden, wenn diese eine Interpretation der Subjekt-Prädikat-Struktur bzw. alltagssprachlicher Identifikationsmechanismen abgibt. Eine Ontologie, die etwa Dinge und ihre Eigenschaften annimmt, bzw. Ereignisse als von Dingen abhängige Entitäten erachtet, kann damit auch als adäquate Deutung der Existenzvoraussetzungen alltäglicher (Sprech-)Praxis gelten.

Alltägliche Praxis ist keine statische Größe, weder synchron, d.h. im Vergleich gegenwärtig etablierter Kulturen, noch diachron, d.h. in historischer Perspektive. Daraus ergibt sich die Annahme, dass auch die Existenzvoraussetzungen alltäglicher Praxis revidier- und entwickelbar sind. Das besagt natürlich nicht, dass es nicht einen Kern kultur- und geschichtlich invarianter Strukturelemente der ontologischen Voraussetzungen alltäglicher Praxis gibt. Das besagt aber, dass man bei einer Reflexion auf diese Voraussetzungen bzgl. dessen, was man im Alltag als Existierendes annimmt, mit Varianz zu rechnen hat, die nicht nur die „Peripherie“, sondern, zumindest theoretisch, auch deren Kern betrifft. Eine Theorie der Existenzvoraussetzungen alltäglicher Praxis muss sich somit auch als entwickel-, ja revidierbar erweisen.

Kurzum: Für eine Theorie der Existenzvoraussetzungen alltäglicher Praxis sind die Kriterien für Alltagsontologie maßgeblich.

Einen Schritt weiter gehen wir, wenn wir uns vor Augen führen, dass wir natürlich auch speziellere Praktiken, als es die alltägliche Praxis ist, in ihren Voraussetzungen hinsichtlich Existenz und Existierendem untersuchen können. Schon zu Beginn wurde in diesem Zusammenhang einzelwissenschaftliche Praxis, etwa jene in den Naturwissenschaften, erwähnt. Ontologie als Universaltheorie über alles, was es gibt, was existiert, kann man nun so betreiben, dass man den Existenzvoraussetzungen alltäglicher Praxis rahmengebenden Status gewährt. Oder man kann Ontologie so in Gang setzen, dass man diesen Rang den Existenzvoraussetzungen einer bestimmten Praxis, etwa einer einzelwissenschaftlichen, zubilligt. Es macht nun aber einen funda- 
mentalen Unterschied, ob man bei seiner Theorie der Grundelemente der Wirklichkeit den ersten Weg oder den alternativen zweiten geht. Im ersten Fall nimmt man an, dass die Existenzvoraussetzungen der alltäglichen Praxis in einer bestimmten Weise grundlegender sind als jene einzelwissenschaftlicher Praxis. Die Existenzvoraussetzungen unseres alltäglichen Lebens geben den Rahmen für einzelwissenschaftliche Praxis und sind maßgeblich für die ontologische Interpretation einzelwissenschaftlicher Ergebnisse. Im zweiten Fall wird man versuchen, aus den Existenzvoraussetzungen einzelwissenschaftlicher Praxis, heute sind damit meistens jene der Physik gemeint, das zu rekonstruieren, was wir im Alltag als Existierendes annehmen. Damit ist eine prioritäre Erklärungskompetenz der Physik im Hinblick auf Existenzannahmen postuliert; um den Preis, dass wir unser alltägliches Verstehen unserer Welt, ja von uns selbst, im „Licht“ der Physik revidieren müssen.

Alltagsontologie ist nichts anderes als der erste Weg, während der zweite die Vorgangsweise revisionärer Metaphysik oder Ontologie charakterisiert. ${ }^{164}$ Für Alltagsontologie sind die Existenzvoraussetzungen unserer alltäglichen Praxis rahmengebend und damit grundlegend für das Verstehen der Existenzvoraussetzungen jeder, auch einzelwissenschaftlicher Praxis. Insofern die systematische Entfaltung dieser Existenzvoraussetzungen alltäglicher Praxis nach den Kriterien der Intuitivität, Entsprechung zu den Grundstrukturen alltäglichen Sprechens, Entwickel- und Revidierbarkeit geschieht, sind diese drei Kriterien auch maßgeblich für eine rahmengebende Universaltheorie bzgl. Existierendem. Damit sollte der Ontologiebegriff des ersten Kapitels in Bezug gebracht sein mit den sich an Strawsons deskriptiver Metaphysik orientierenden Kriterien von Alltagsontologie.

Gegen Ende des Abschnitts 3.12, in dem die genannten Kriterien für Alltagsontologie entwickelt wurden, war davon die Rede, dass das Betreiben von Alltagsontologie auf einer Bewertung zu beruhen hat. Diese Bewertung besteht darin, dass Alltagsontologie gegenüber revisionärer Ontologie der Vorrang zu geben ist. Dieser Gedanke soll an dieser Stelle unterstrichen werden. Alltagsontologie ist nicht die Wahl einer, relativ zu ihrer Alternative gleich-

\footnotetext{
${ }^{164}$ Nota bene: Damit wird nicht behauptet, dass nicht auch revisionäre Ontologie allgemein, d.h. methodisch unabhängig von Einzelwissenschaften vorgeht. Sie ist Ontologie; allerdings eine, die von der Priorität der Existenzvoraussetzungen einer Einzelwissenschaft ausgeht.
} 
wertigen Interpretation der Existenzvoraussetzungen menschlicher Praxis. Der Vorzug der Alltagsontologie ist keine Sache des „Geschmacks“ oder pragmatisch anzunehmen. Eine solche Bewertung verlangt vielmehr eine $B e$ gründung. Wie gesagt: Einem Alltagsontologen muss es darum gehen, Argumente vorzubringen, warum eine sich am alltäglichen Verstehen der Wirklichkeit orientierende Ontologie gegenüber einer naturwissenschaftlich ausgerichteten der Vorzug zu geben ist.

Diese Begründung soll nun in gebotener Ausführlichkeit im nächsten Teil dieser Arbeit erfolgen. Wie in der Einleitung zu diesem dritten Kapitel angekündigt, wollen wir uns an jenen allgemeinen Einwänden gegen die Ontologie orientieren, wie sie in den Abschnitten 2.1-2.3 unter den Stichwörtern „Deflationierung“, „Naturalisierung“, „Simplifizierung“ vorgebracht wurden. Zentral ist dabei die Auseinandersetzung mit dem Naturalismus, weil dieser, mitunter sogar explizit, als Gegenprogramm zu deskriptiver bzw. Alltagsontologie entwickelt wird. Ob jeder Naturalismus im Sinne revisionärer Ontologie verstanden werden muss, sei dahingestellt. Faktisch laufen die einflussreichsten naturalistischen Strömungen aber auf eine Revision alltäglichen Sprechens, Denkens, Weltverstehens hinaus. Auch der Simplifizierungsvorwurf ist in einer Begründung des prioritären Status von Alltagsontologie zu berücksichtigen, weil, wie wir gesehen haben, dieser Vorwurf vorwiegend nicht als allgemeine Kritik an der Ontologie zu verstehen ist, sondern hauptsächlich eben an Alltagsontologie. Alltagsontologie sei Ausdruck naiver Theorienbildungen über die Grundstrukturen der Wirklichkeit. Dem gilt es zu entgegnen. Begonnen soll allerdings mit dem zuerst Genannten werden: Der Frage, wie man sich als Alltagsontologe mit den Kritikpunkten aus dem „Deflationierungslager" auseinandersetzen kann. Es wird zu zeigen sein, dass gerade eine differenzierende Auseinandersetzung mit AutorInnen aus dieser Positionierung zur Weiterentwicklung von Alltagsontologie beitragen kann, auch unter Rücksicht der argumentativen Darlegung ihres grundlegenden Charakters gegenüber revisionärer Ontologie. 


\subsection{Alltagsontologie und Ontologiekritik}

Bevor der am Ende des letzten Abschnitts gelegte Faden aufgegriffen wird, soll, bei dieser Gelegenheit des Einstiegs in einen neuen Themenbereich, kurz der Blick auf den weiteren Kontext des nunmehr Folgenden gerichtet werden. Worum es in diesem Teilband des Gesamtprojekts einer Darstellung der Ontologie geht, ist, Alltagsontologie in Auseinandersetzung mit aktuellen metaontologischen Debatten zu entwickeln. Nach der Einführung eines ersten Ontologiebegriffs (Kapitel 1.), der Darlegung von Ontologiekritik aus der aktuellen Metaontologie (Kapitel 2.), geht es nunmehr um die Etablierung der Ontologie als Alltagsontologie (Kapitel 3.). Ziel wird es sein, Alltagsontologie so weit auf Schiene zu bringen, dass sie als Rahmenprogramm für die Ausarbeitung eines kategorial-ontologischen Schemas fungieren kann. Dies wird im zweiten Teilband geschehen. Natürlich nicht, und damit komme ich wieder zurück zur nunmehrigen Aufgabe, ohne dass die sachliche Auseinandersetzung mit der Ontologiekritik des zweiten Kapitels erfolgt. Dabei kann es nicht um reine Apologetik gehen, sondern vielmehr darum, im Diskurs mit metaontologischen Positionen die Eigenart der Alltagsontologie in aufbauender Absicht herauszuarbeiten und ihren Vorzug gegenüber revisionären Theorien über die Grundstrukturen der Wirklichkeit zu begründen.

In diesem Sinn soll nun, wie angekündigt, zunächst der Dialog mit dem Deflationierungsprogramm gesucht werden.

\subsection{Alltagsontologie und Deflationierung}

Wie im einschlägigen Abschnitt (2.1) über den metaontologischen Deflationismus (engl.: deflationism) gezeigt wurde, handelt es sich dabei um ein „Bereinigungsprogramm“, das der Ontologie verordnet wird, um von vermeintlichen Themen und Fragestellungen zu den tatsächlich für sie relevanten zu kommen. Am radikalsten ist die Position, dass es sich bei den standardmäßig in der Ontologie diskutierten Fragen gerade nicht um solche über die Grundstrukturen der Wirklichkeit handelte, sondern um rein konzeptuelle Probleme, letztlich um Streitereien um Worte. Weniger radikal ist die Auffassung, dass die Deflationierung im Fall der Ontologie darin zu bestehen habe, vermeintlich schwere Probleme überzuführen in leichte (engl.: easy), z.B. in eine 
Analyse von Begriffen, insbesondere von deren Anwendungsbedingungen. Schließlich lassen sich auch jene Positionen unter das Deflationierungsprogramm subsumieren, welche das tiefe Problem von Sein und Existenz auf die Frage nach der Bedeutung des Begriffs „Existenz“bzw. des Existenzquantors zurückführen. Wie, um die Grundfrage dieses Abschnittes aufzugreifen, können wir diese kritischen Einstellungen auf Alltagsontologie beziehen, vielleicht sogar für die Weiterentwicklung derselben fruchtbar machen?

\section{Streit um Worte}

Bleiben wir, in Analogie zur Gliederung des Abschnitts 2.1, zunächst bei der radikalsten Version, beim Versuch, die Ontologie auf einen reinen Streit um Worte zu deflationieren. Zunächst sei zugestanden, dass dieser Vorschlag gegen jede Ontologie gerichtet ist, also auch unsere Alltagsontologie betrifft. Wollen wir Alltagsontologie betreiben, ist also Entgegnung bzw. Zurückweisung der Streit-um-Worte-Position nötig.

Wir können dabei bei der gegebenen allgemeinen Charakterisierung von Streiten um Worte beginnen. Diese besteht darin, dass Sprecher und Sprecherinnen bei Streiten um Worte (allein) deshalb in Konflikt geraten, weil sie bzgl. der Bedeutung mancher sprachlicher Ausdrücke voneinander abweichen: ,[...] speakers disagree because they semantically deviate from each other [...]“. ${ }^{165}$ Das ,,allein“ in der Parenthese oben ist so $\mathrm{zu}$ interpretieren, dass, über die semantische Nichtübereinstimmung hinaus, kein sachlicher Gegensatz vorliegt. Das ist der Fall, wenn nach einer Bedeutungsklärung (i) ein/e OpponentIn den Sprachgebrauch des/der jeweils anderen anerkennen kann, dazu (ii) dessen/deren inhaltlichen Standpunkt relativ zu diesem Sprachgebrauch, ohne (iii) dabei eigene Überzeugungen aufgeben zu müssen.

Sind alle ontologischen Dispute auf diese Weise aufzulösen, wird die Ontologie insgesamt auf einen Streit um Worte zurückgeführt. Wie kann dies speziell auf Alltagsontologie bezogen werden? Dazu gibt es prima facie drei Möglichkeiten. Die erste ist, den Streit-um-Worte-Verdacht auf Debatten zu beziehen, ob eine These im Kontext einer Alltagsontologie adäquater ist als eine andere. Ist im Falle des Vorliegens zweier konkurrierender Thesen T1 und T2, T1 die angemessenere Interpretation der Existenzvoraussetzungen

${ }^{165}$ Manley 2009, 14. 
unserer alltäglichen Praxis, oder T2? Wenn dies allein auf einen Streit um Worte, in eingeführtem Sinn, zurückzuführen ist, kann Alltagsontologie als solche deflationiert werden. Die zweite Möglichkeit, den Streit-um-WorteEinwand in Bezug zu Alltagsontologie zu setzen, ist, die Frage, ob eine These (überhaupt) eine alltagsontologische ist, rein semantisch aufzulösen. Schließlich, Möglichkeit drei, kann auch die Grundsatzfrage, ob revisionärer Ontologie oder Alltagsontologie der Vorzug zu geben sei, dem Streit-um-WorteVerdacht ausgesetzt werden. Unser Projekt kann demnach auch so deflationiert werden, dass der Disput über die Priorisierung der Existenzvoraussetzungen alltäglicher Praxis gegenüber jenen einer einzelwissenschaftlichen Praxis auf einen semantischen Meinungsunterschied zurückgeführt wird.

Bevor wir hier alle drei Ebenen mit einiger Vehemenz verteidigen, tun wir gut daran einzuräumen, dass es auch im Kontext von Alltagsontologie manche Debatten geben mag, die durch eine semantische Klärung der verwendeten Begrifflichkeiten entschärft, wenn nicht sogar aufgelöst werden können. Gerade die als erste Möglichkeit angeführten Streitfälle bzgl. der „,besseren“ Alltagsontologie, das ist die adäquatere Auslegung der Existenzvoraussetzungen alltäglicher Praxis, bieten sich hier an. Warum sollte es nicht auch AlltagsontologInnen möglich sein, beim Disput über zwei alternative Thesen zur Auslegung von Existenzvoraussetzungen alltäglicher Praxis dazu zu kommen, die Unterschiedlichkeit ihrer Positionen allein auf Differenzen in der Bedeutung sprachlicher Ausdrücke zurückzuführen; so dass die jeweils andere Bedeutungspräferenz akzeptabel erscheint (im Sinne von (i)), die fragliche These des Opponenten/der Opponentin relativ dazu annehmbar ist (ii), ohne dass damit die je eigene These aufgegeben werden müsste (iii)?

Die Frage ist allerdings, ob dies für alle Debatten auf dieser ersten Ebene, darüber hinaus auch auf der zweiten und dritten gilt. Dies freilich kann in Zweifel gezogen werden.

Schon auf der zitierten ersten Ebene übrigens. Das ergibt sich daraus, dass sich der Konfliktfall, ob eine These T1 oder die ihr entgegenstehende These T2 die adäquatere Interpretation der Existenzvoraussetzungen alltäglicher Praxis ist, nicht in jedem Fall als rein semantischer rekonstruieren lässt. Um von den Beispielen bzgl. diachron identischer Dinge wegzukommen, können 
wir uns einer Frage im Kontext des guten alten Universalienstreits zuwenden. Nehmen wir an, auf einem Tisch liegen drei rote Kugeln. Im Hinblick auf das Problem, wieviele Rot-Eigenschaften hier vorliegen, könnte man nun die These (nennen wir sie T1) vertreten, dass hier eine universale Röte dreimal vorkommt. Desgleichen lässt sich die Auffassung vertreten (bezeichnen wir sie als T2), dass hier drei klar umrissene Rot-Partikularien verschiedene $\mathrm{Ku}$ geln charakterisieren. Nehmen wir nun an, dass sowohl T1 als auch T2 beanspruchen, eine Interpretation bestimmter Existenzvoraussetzungen alltäglicher Praxis zu sein. Damit stehen wir vor der Frage, welche nun tatsächlich die angemessenere ist. Nota bene: Hier geht es natürlich nicht um eine Lösung des Universalienproblems. Wir haben uns auf die Untersuchung zu beschränken, ob die besagte Frage in die wiederholt geschilderte Streit-umWorte-Konstellation mündet; sprich, dass nach Klärung der verwendeten termini technici (etwa „Universale“, „Partikulare“, „Eigenschaft") der Sprachgebrauch der jeweils anderen Partei annehmbar im Sinne von (i) ist, man relativ dazu die jeweils andere These als adäquate Interpretation von alltäglichen Existenzvoraussetzungen akzeptieren kann (ii), ohne seine eigene These, siehe (iii), als alltagsontologische aufgeben zu müssen. Das ist schlicht zu verneinen. Als alltagsontologischer Freund der Drei-Eigenschaften-Position, also von T2, kann ich keinen T1-Sprachgebrauch akzeptieren, der auf dem besagten Tisch lediglich eine Röte annimmt (siehe (i)), relativ dazu eine Interpretation der Existenzvoraussetzungen alltäglicher Praxis, welcher so etwas wie eine universale Eigenschaft, die an drei Kugeln vorkommt (ii); zumindest nicht, ohne meinen eigenen, nominalistischen Standpunkt als alltagsontologischen über Bord werfen $\mathrm{zu}$ müssen (iii). Kurzum: Nach einer durchaus möglichen Begriffsklärung beginnt der Konflikt erst, er löst sich nicht auf.

Ein möglicher Einwand mag darauf rekurrieren, dass die besagte Unversöhnlichkeit von T1 und T2 im Beispiel auf einen tiefer liegenden ontologischen Konflikt zurückzuführen und nicht spezifisch alltagsontologisch ist. Dazu sei vermerkt, dass die Spitze dieses Einwands umgekehrt und zugunsten der These angeführt werden kann, dass sich Dispute über die angemessenere Interpretation alltäglicher Praxis im Hinblick auf Existierendes nicht als Streit um Worte deflationieren lassen: Weil und insofern es ontologische 
Auseinandersetzungen, wie das Universalienproblem, gibt, die sich nicht im Sinn der Streit-um-Wort-Partei auflösen lassen, lassen sich auch Debatten über die bessere Alltagsontologie nicht als Streite um Worte rekonstruieren, wenn beide Parteien, im Beispielsfall bzgl. der Existenz von Universalien, ihre Thesen als Interpretationen alltäglicher Praxis verstehen.

Wir können uns damit der zweiten angeführten Möglichkeit zur Deflationierung zuwenden. Hier können wir insbesondere auf die Anwendung unserer Alltagsontologie-Kriterien Intuitivität, Entsprechung zu Grundstrukturen alltäglichen Sprechens und Entwickel- und Revidierbarkeit rekurrieren. Diese entscheiden, ob eine These eine alltagsontologische ist, oder eben nicht. Der springende Punkt liegt darin, dass eine Untersuchung, $o b$ eine These diesen Kriterien entspricht, gerade kein semantisches Unterfangen ist. Natürlich tun wir gut daran, im Konfliktfall unsere Begriffe zu klären. Ist das geschehen, sind wir allerdings nicht am Ende, sondern erst am Beginn der maßgeblichen Prüfung, z.B. ob eine These intuitiv nachvollziehbar ist, oder eben nicht. Desgleichen ist die Frage, ob eine These im Kontext einer plausiblen Interpretation der Subjekt-Prädikat-Struktur bzw. des Identifikationsapparats unseres alläglichen Sprechens vertreten werden kann, natürlich eine auf sprachliche Grundzüge bezogene. Entschieden werden kann sie jedoch durch die Klärung der in ihr verwendeten Begriffe nicht. Dass z.B. eine Ontologie mit Dingen und Ereignissen als von Dingen abhängigen Entitäten im Zusammenhang mit der Erläuterung des faktischen Identifikationsapparats unserer Alltagssprache vertreten werden kann, bezieht sich auf diese Grundstruktur unseres Sprechens. Wie es aber zu verstehen ist, dass dem so ist, lässt sich allein mit einer Klärung des Ding- bzw. Ereignisbegriffs nicht darlegen. Umso mehr gilt das Ungenügen rein semantischer Analysen für die Klärung der Frage, ob eine These dem spezifischen Entwickel- bzw. Revidierbarkeitskriterium von Alltagsontologie entspricht.

Die dritte erwähnte Ebene der Diskussion bietet besondere Chancen, den Streit-um-Worte-Verdacht auf Alltagsontologie zu beziehen, bzw. auch für das Projekt Alltagsontologie, vom Streit-um-Worte-Einwand zu profitieren. Führen wir uns dazu nochmals eines der im Abschnitt 2.11 angeführten „Problem-Probleme“ vor Augen, nämlich das spezielle Kompositionsproblem. Steht hier ein Ding der Art Sessel, oder handelt es sich dabei nur um ses- 
sel-weise arrangierte Atome oder simples? Diesen Streit könnte man (nur) dann im Sinn der Streit-um-Worte-Position auflösen, wenn man durch eine semantische Klärung der verwendeten Begriffe, etwa „Sessel“ bzw. „Atom“, so weit zu einer Streitschlichtung käme, dass die eine Partei den Sprachgebrauch der jeweils anderen anerkennt (i), sowie die alternative Position als relativ zu diesem Sprachgebrauch zutreffend zugestehen könnte (ii), ohne dabei ihren eigenen inhaltlichen Standpunkt aufgeben zu müssen (iii).

Wie in 2.11 gesehen, zeigt eine genauere Analyse, dass dies schlicht nicht der Fall ist. Die Akzeptanz des ,gegnerischen“ Sprachgebrauchs (i) steht unter einer Bedingung, deren Erfüllung von weitreichenden theoretischen Zugeständnissen abhängt: van Inwagen hat Recht, wenn die Einheits- und Kompositionsfrage für Artefakte im Allgemeinen nicht geklärt werden kann; wenn es so etwas gibt wie metaphysische Atome (engl.: simples), deren Agglomeration u.a. Sessel bilden. Die ,wenn dem so sein mag“-Klärung zeigt die Voraussetzungen bzw. den theoretischen Rahmen eines Sprachgebrauchs, der von einem Artefakte-freundlichen Anti-Atomisten gerade nicht geteilt werden kann (gegen (i)), somit auch nicht einzelne Thesen relativ zu diesem Sprachgebrauch (das steht (ii) und (iii) entgegen). Hier endet also nicht der Streit, vielmehr beginnt er. Das ontologische Problem, ob Sessel nicht doch Grundelemente der Wirklichkeit sind, der Atomismus aber falsch ist, wird durch die semantische Klärung von „Sessel“ bzw. „Atom“ nicht gelöst, schon gar nicht aufgelöst.

Das gegebene Beispiel ist deshalb gewählt bzw. an dieser Stelle aufgegriffen, weil es den exemplarischen Fall eines Disputs darstellt, der in seinen, durch eine „wenn dem so sein mag“-Analyse aufgedeckten Voraussetzungen auf die Alternative Alltagsontologie bzw. revisionäre Ontologie verweist. Die Akzeptanz von metaphysischen Atomen oder simples ist geradezu paradigmatisch revisionär, während die Annahme von Sitzmöbeln und anderen Artefakten als Grundelemente der Wirklichkeit den unvermeidbaren Bestandteil einer tauglichen Interpretation der Existenzvoraussetzungen alltäglicher Praxis bildet. Wenn nun also der Streit, ob Sessel Entitäten sind oder nicht doch van Inwagens Atomismus wahr ist, nicht semantisch (auf-)gelöst werden kann, so auch nicht die damit einhergehende Entscheidung bzgl. des Vorzugs entweder der Alltagsontologie oder einer revisionären Ontologie. Wir sind 
bei der Entgegnung des Streit-um-Worte-Einwands wider die Alltagsontologie auf der dritten Ebene angelangt.

Damit ist auch jener Gesichtspunkt angesprochen, von dem eingangs als Profit der Alltagsontologie vom Streit-um-Worte-Einwand die Rede war. Auseinandersetzungen um das spezielle Kompositionsproblem im Hinblick auf Artefakte zeigen nämlich, dass man manche Streite möglicherweise doch bereinigen kann; allerdings nicht im Sinne der Auflösung eines ontologischen Disputs in einen Streit um Worte, sondern in einen Streit, ob Alltagsontologie oder revisionäre Ontologie die adäquatere Interpretation der Rahmenbedingungen unserer Praxis, umfassend verstanden, ist. Das Kompositionsproblem wird nicht zu einem semantischen Problem degradiert. Seine Rekonstruktion zeigt aber, dass es im Kern auf einen Disput hinausläuft, ob man den Existenzvoraussetzungen alltäglicher Praxis im Hinblick auf eine ontologische Universaltheorie den Vorzug zu geben hat, oder den Existenzvoraussetzungen bestimmter Einzelwissenschaften. Ersteres impliziert die Annahme der Existenz von Artefakten; Letzteres ist, bereinigt gesehen, die entscheidende Voraussetzung z.B. atomistischer Ontologie.

\section{Easy Ontology}

Wir können damit zum zweiten, vielleicht weniger radikal anmutenden Ansatz im Kontext des Deflationierungsprogramms kommen, das ist die „Bereinigung“ der Ontologie um die schweren, hin zu ausschließlich leichten Fragen. Im Abschnitt 2.12 wurde die Easy Ontology von Amie Thomasson unter dieser Rücksicht diskutiert. Dies soll hier aufgegriffen und auf Alltagsontologie angewendet werden. Bei dieser Anwendung können wir zunächst auf bestimmte augenfällige Überlappungsflächen zwischen der Easy Ontology und Alltagsontologie, wie sie hier konzipiert wurde, verweisen. Dann aber gilt es auch Linien zu markieren, die eine Abgrenzung zwischen diesen Ansätzen ermöglichen. Schließlich soll auch in diesem Zusammenhang die Frage gestellt werden, welche Anliegen der Easy Ontology für die Weiterentwicklung einer Alltagsontologie fruchtbar gemacht werden können; bzw. inwieweit die kritische Auseinandersetzung mit Amie Thomasson auch konstruktiv zum Aufbau der Alltagsontologie von Nutzen sein mag. 
Beginnen wir mit den angesprochenen gemeinsamen Anliegen. Ein wesentlicher Aspekt in diesem Zusammenhang kann mit „Naturalismuskritik“ überschrieben und auf eine Kritik an Quine pointiert umgelegt werden. Zur Erinnerung: Thomasson kritisiert Quine als Vorreiter einer „dogmatischen“ (folglich „schweren“) Metaphysik, insofern er, die Arbeitsteilung zwischen Naturwissenschaften und Philosophie missachtend, sich daran macht, wie in den Naturwissenschaften, bestimmte „matters of fact" aufzufinden. Dabei verkenne er die Kernaufgabe der Philosophie, die nach Thomassons Meinung in Begriffsanalyse besteht.

Es kann hier nicht die Aufgabe sein zu untersuchen, welche Gesichtspunkte von Thomassons Quine-Kritik nun zutreffend sind, welche aber nicht. Insbesondere können wir die Frage ausklammern, ob Quine in seiner Theorie ontologischer Verpflichtungen naturwissenschaftlicher Theorien tatsächlich einen tendenziell so naiven Realismus annimmt, wie Thomasson ihm das zu unterstellen scheint, Stichwort „Dogmatismus“; oder ob insbesondere seine Auffassung von physikalischen Objekten (engl.: physical objects) als Setzungen (engl.: posits) nicht doch auch einen pragmatisch, somit nicht-dogmatisch orientierten Gesichtspunkt in der Interpretation naturwissenschaftlich aufgewiesener empirischer Daten mit einbringt.

Bemerkenswert ist jedenfalls der Ansatz von Thomassons Naturalismuskritik, der wie gesagt darin besteht, dass es zwischen Naturwissenschaft und Philosophie, ergo auch der Ontologie, eine Arbeitsteilung gibt, die keinesfalls durch eine methodische Ineinssetzung oder gar eine inhaltliche Ausrichtung der Ontologie an den Naturwissenschaften verwischt werden darf. Ontologie ist nicht Naturwissenschaft und kann nicht a posteriori betrieben werden. Das können wir von Thomassons Easy Ontology annehmen und als Gemeinsamkeit mit der Alltagsontologie betrachten. Es steht ja im Kern alltagsontologischer Überzeugungen, dass die Praxis einer Einzelwissenschaft und deren ontologische Voraussetzungen nicht zum Maßstab einer allgemeinen und universalen Theorie von Existenzvoraussetzungen menschlicher Praxis gemacht werden kann. Es ist vielmehr die alltägliche Praxis, bzw. die Reflexion darauf im Hinblick auf deren Voraussetzungen bzgl. Existierendem. Bemerkenswerterweise bringt Amie Thomasson diesen Bezug auf die alltägliche Praxis und deren zentralen Stellenwert auch explizit zum Ausdruck, gerade 
unter der hier maßgeblichen Rücksicht. Existenzfragen, so Thomasson, können nicht auf Fragen nach ontologischen Verpflichtungen einzelwissenschaftlicher Theorien reduziert werden. Existenzfragen sind zu stellen mit Bezug auf das, was wir in unserem Alltag praktisch tun. ${ }^{166}$

Wenngleich die Easy Ontology beachtliche Übereinstimmungen mit alltagsontologischen Auffassungen aufweist, ist die Alltagsontologie keine Easy Ontology. Letztere ist ein neo-carnapianisches Programm, Erstere nicht; ohne dass mit dieser Behauptung jedwede Berührungspunkte mit Carnap ausgeschlossen werden müssten. Im Kern lässt sich die Grenzziehung an der Einstellung zur kognitiven Relevanz ontologischer Fragen festmachen. Die Entscheidung von Existenzfragen ist für Thomasson, ebenso wie für Carnap, teilweise „easy“, teilweise rein pragmatisch anzugehen. Bei internen Existenzfragen geht es schlicht darum abzuklären, ob die Applikationsbedingungen von bestimmten sprachlichen Ausdrücken erfüllt sind, oder nicht. Das kann entweder einfach empirisch geschehen, oder logisch, je nach der Eigenart jenes begrifflichen Rahmens, dem die fraglichen Ausdrücke angehören. Die externe Wahl des begrifflichen Rahmens selbst aber ist bei Carnap pragmatisch zu entscheiden. Bei Thomasson ist dies, so haben wir im Abschnitt 2.12 gesehen, ${ }^{167}$ ebenfalls vorausgesetzt. In der Alltagsontologie können wir eine solche exhaustive Unterscheidung zwischen pragmatisch und „leicht“, sprich empirisch oder logisch, zu entscheidenden Existenzfragen nicht annehmen. Was die Voraussetzungen alltäglicher Praxis im Hinblick auf Existierendes sind, ist ganz bestimmt nicht leicht zu entscheiden, weder durch eine empirische Untersuchung im Sinne eines carnapianisch-verifikationistischen Programms, noch logisch. Ferner sind die begrifflichen Rahmenbedingungen, in denen wir dann eine alltagsontologische Theorie entwickeln, natürlich auf Praxis bezogen. Das heißt freilich nicht, dass die Wahl dieser Bedingungen nach pragmatischen Kriterien im Sinne Carnaps erfolgen kann. Da gibt es vielmehr Gründe anzuführen, warum etwa diese Rahmenbedingungen in der Lage sind, die Existenzvoraussetzungen alltäglicher Praxis zu reflektieren, jene aber nicht. Ist, um zu unserem Standardbeispiel zurückzukehren, eine endurer-Ontologie besser geeignet, die Existenzvoraussetzungen alltäglicher

\footnotetext{
${ }^{166}$ Siehe die einschlägigen Ausführungen in 2.12, besonders die Textverweise in Fußnote 27. 167 ... v.a. dort, wo es um pragmatische Voraussetzungen der easy inferences ging.
} 
Praxis zu klären, oder eine perdurer-Theorie? Die Gründe für einen Standpunkt haben sich, wie in den letzten Abschnitten gesehen, an Kriterien zu orientieren. $\mathrm{Ob}$ diese erfüllt sind, darüber kann man nun, auch das haben wir eben erst in der Erörterung der Streit-um-Worte-Position gesehen, diskutieren. Diese Diskussion aber ist unter Anführung von Argumenten zu führen und somit kognitiv relevant.

Damit zusammenhängend können wir es als ein grundlegendes Unterscheidungsmerkmal zwischen Easy Ontology und Alltagsontologie annehmen, dass Erstere ausschließlich die Erfüllung von Applikationsbedingungen von Termini als Existenzkriterium annimmt. Alltagsontologie kann sich keinesfalls darauf reduzieren. Das kann an dieser Stelle, an der es um Abgrenzung geht, behauptet werden, ohne dass positiv erläutert werden müsste, wie die Entfaltung des zentralen alltagsontologischen Existenzkriteriums, d.i. zu den Voraussetzungen alltäglicher Praxis bzgl. Existierendem zu gehören, vorzunehmen ist. Das soll Thema späterer Abschnitte sein, v.a. auch jener Teile, in denen es um die Ausarbeitung eines kategorialen Rahmens nach alltagsontologischen Voraussetzungen geht. Jedenfalls ist klar, dass Begriffsanalyse im Sinne der Easy Ontology allein nicht zur Klärung von Existenzvoraussetzungen unserer Praxis genügt, wir vielmehr auch „schwerere“ Kriterien in Anschlag bringen werden müssen. Weder ist alltägliche Praxis auf Begriffsverwendung zu reduzieren, noch ist die Untersuchung der Erfüllung von Applikationsbedingungen von Termini die einzige Möglichkeit, Existenzvoraussetzungen irgendeiner Praxis zu klären.

Analoges gilt für die Beschränkung ontologischer Fragen auf Existenzfragen. In einer Alltagsontologie kann es nicht nur darum gehen, was faktisch zu den Voraussetzungen alltäglicher Praxis bzgl. Existierendem zu zählen ist, sondern (u.a.) auch darum, wie die so anzunehmenden Entitäten ontologisch zu charakterisieren sind, ob es Entitäten gibt, die sich in ihren grundlegenden Merkmalen voneinander unterscheiden, bzw. wie sich Entitäten verschiedener Kategorien zueinander verhalten. Auch wird sich zeigen, dass die Klärung der Existenzvoraussetzungen alltäglicher Praxis nicht mit einer Auflistung eines Kategorienschemas das Auslangen finden wird. Für jetzt ist entscheidend: Alltagsontologie ist mehr als die Klärung von Existenzfragen. 
Insofern das für die Easy Ontology nicht gilt, ist Alltagsontologie von ihr zu unterscheiden.

Damit kommen wir aber auch schon zum dritten angekündeten Gesichtspunkt unserer Bezugsetzung von Easy Ontology und Alltagsontologie: Was können wir als AlltagsontologInnen von der Diskussion mit der Easy Ontology lernen, und zwar für den Aufbau unseres eigenen Programms?

Die neo-carnapianische Differenzierung zwischen „leichter“ und „schwerer" Ontologie kann im Rahmen einer Alltagsontologie nicht übernommen werden. Und dennoch: Sie weist auf eine Unterscheidung hin, die für eine Alltagsontologie von einiger Bedeutung ist und hier eigenständig entwickelt werden soll. Gemeint ist die Unterscheidung zwischen einer „dogmatischen“ Ontologie $^{168}$, die meint, unabhängig von menschlicher Praxis Grundstrukturen einer „Wirklichkeit an sich“"169 theoretisch abbilden zu können und einer, nicht pragmatischen (!), aber sich an der Praxis orientierenden Analyse dessen, was als seiend oder existierend anzunehmen ist. Man könnte diese Unterscheidung auch darlegen als jene zwischen einer stark- oder absolut-realistischen Interpretation der Wirklichkeit und einem, nicht relativistischen (!), sondern relativ-realistischen Verstehen der Welt. Ersterem zufolge liegt das, was existiert, unabhängig von oder vorab zu jedweder menschlichen Praxis vor und ist in einer ontologischen Theorie bestmöglich abzubilden. In Theodore Siders ,Writing the Book of the World“ kann man, wie bereits dargelegt, eine solche Einstellung paradigmatisch vorfinden. Die Metapher der Landkarte kann hier hilfreich sein. Beim Buch-der-Welt-Schreiben geht die Ontologin bzw. der Ontologe vor wie ein Kartograph, der die Umrisse der vorab seinem Tun vorliegenden geographischen Strukturen nachzeichnet. Alltagsontologie setzt sich davon ab; ohne freilich, das muss sofort betont werden, in das gegenteilige Extrem zu verfallen: Alltagsontologie ist kein metaphysischer Idealismus. Der handelnde Mensch bringt die Wirklichkeit nicht hervor. Die Welt existiert ,,an sich“. Das ergibt sich schon daraus, dass man aus idealistischen oder gar solipsistischen Grundannahmen menschliche

\footnotetext{
168 ... in freier Anlehnung an Kants „Schlummer“, aus dem ihn Hume wohl befreite. Vgl. ders. 1968b, Vorrede zu den „Prolegomena“, 260.

169 ... ebenso.
} 
Praxis gar nicht rekonstruieren könnte. ${ }^{170}$ Wie aber die Welt ist, wie ihre Grundelemente zu bestimmen sind bzw. wie sich diese zueinander verhalten, kann nach alltagsontologischen Annahmen nur in einer Reflexion auf, also relativ zu jenen Voraussetzungen bzgl. dieser Grundelemente und ihrer Verhältnisse herausgefunden werden, die wir in unserer alltäglichen Praxis machen. Dieser nicht-dogmatische Charakter der Alltagsontologie kommt besonders in jenem Kriterium für Alltagsontologie zum Ausdruck, das auf die Entwickel- und Revidierbarkeit ihrer Theorien rekurriert. Insofern sich menschliche Praxis entwickelt, so auch die Ergebnisse einer systematischen Reflexion auf ihre Existenzvoraussetzungen.

Wir sehen also: Die Distinktion zwischen ,leichter“ und „schwerer“ Ontologie kann weiterentwickelt werden in Richtung Unterscheidung zwischen „praxisbezogenen“ und „dogmatischen“, wenn man so will zwischen naiven absolut-realistischen Verständnisweisen der Wirklichkeit und reflektierten relativ-realistischen Interpretationen.

Eine weitere neo-carnapianische Distinktion, die von Amie Thomasson an verschiedenen Stellen aufgegriffen wird, ist die zwischen der Erwähnung (engl.: mentioning) und der Verwendung (engl.: using) sprachlicher Ausdrücke. Bei externen Fragen erwähnen wir bestimmte Begrifflichkeiten, um sie pragmatisch im Hinblick auf ihre Nützlichkeit für theoretische Kontexte zu untersuchen. Dabei legen wir, im Bedarfsfall, auch ihre Applikationsbedingungen fest. Bei internen Fragen wiederum verwenden wir diese Termini, um im konkreten Fall die Erfüllung dieser Applikationsbedingungen zu prüfen. Auch dies ist, wie bereits ausgeführt, in einer Alltagsontologie nicht angebracht. Allerdings kann auch diese Distinktion weiterentwickelt werden: In der Alltagsontologie klären wir die Existenzvoraussetzungen von konkreten alltäglichen Vollzügen. Dafür verwenden wir bestimmte Begrifflichkeiten, etwa Ausdrücke für Ereignisse, aber auch für Dinge. Davon zu unterscheiden ist jene systematische Reflexion, welche die dort verwendeten Begriffe erwähnt, um sie im Rahmen einer ontologischen Theorie als termini technici

\footnotetext{
${ }^{170} \mathrm{Vgl}$. dazu jene Autoren in der Positionierung transzendentaler Metaphysik, die, als Bedingung der Möglichkeit diverser menschlicher Vollzüge oder Praktiken, den Ausgriff auf einen Horizont, wie sie sagen, des Seins, also von Existierendem annehmen. Vgl. u.a. Coreth 1999, v.a. Abschnitt 2.
} 
einzuführen und ontologisch zu interpretieren. Bleiben wir bei jenen Vollzügen, die wir schon im Eingangskapitel als Beispiele angeführt haben: Der Wecker läutet, ich stehe auf, nehme einen Arbeitstag in Angriff. Dabei habe ich es zu tun mit lieben Menschen natürlich, u.a. aber auch mit Kaffeemaschine, Tageszeitung, zudem mit einer Busfahrt ins Büro. Bei der Klärung der praxisermöglichenden Vorannahmen bzgl. Existierenden werde ich somit Begriffe verwenden, die sich auf diese Gegenstände beziehen lassen, in unserem Fall u.a. „Kaffeemaschine“ bzw. „Busfahrt“. Das aber ist natürlich nur ein erster Schritt hin zu einer ontologischen Theorie. Ein weiterer ist die Erwähnung dieser Begrifflichkeiten beim Versuch, eine ontologische Theorie zu entwickeln, die u.a. darin bestehen mag, Kaffeemaschine und Busfahrt in ein kategoriales Rahmenwerk zu bringen, welches Vorkommnissen der darin angenommenen Kategorien jene Merkmale zuweist, die dann tatsächlich in der Lage sind, im Sinne einer Alltagsontologie, die Praktiken im Beispielszenario zu erläutern. Kaffeemaschinen sind Dinge, die, als endurer verstanden, bestimmte Eigenschaften aufweisen; Busfahrten aber Ereignisse, die z.B. in bestimmte Abschnitte oder Phasen unterteilt werden können.

Ob diese Explikationen dann tatsächlich alltagsontologisch tauglich sind, zeigt sich u.a. an der Anwendung des Intuitivitätskriteriums, aber auch jenes Kriteriums, das auf die Entsprechung zu Grundstrukturen unseres Sprechens verweist. In gegenständlichem Fall dürften beide, diachron identische Kaffeemaschinen-Dinge, samt ihren erfreulichen Eigenschaften, aber auch Busfahrts-Ereignisse recht gute Chancen haben. Es ist intuitiv durchaus plausibel, die Busfahrt als Ereignis zu verstehen, welches aus bestimmten Abschnitten, etwa Teilstrecken zwischen Stationen, aufgebaut ist; Kaffeemaschinen und ihre Eigenschaften aber als Entsprechungen zur Subjekt-Prädikat-Struktur unserer Alltagssprache.

Auch hier stellen wir fest, dass eine in der Easy Ontology verwendete Unterscheidung kreativ für alltagsontologische Zwecke weiterentwickelt werden kann, und zwar so, dass wir keinesfalls nur Nebensächliches daraus herleiten, sondern grundlegende Merkmale alltagsontologischer Theorienbildung ins Auge fassen. 


\section{Quantorenvarianz}

Wir kommen damit zur dritten behandelten Positionierung des Deflationierungsprogramms, welche von einer Varianz der Bedeutung von „Existenz“ bzw. des Existenzquantors ausgeht, und deshalb kurz mit Quantorenvarianz (engl.: quantifier-variance) betitelt ist. Hier wird in besonderem Maße der Aspekt der Weiterentwicklung der Alltagsontologie zu berücksichtigen sein, zumal, wie gezeigt werden soll, diese Weiterentwicklung auch weitgehend innerhalb der Quantorenvarianz-Theorie vorgenommen werden kann. Es geht also nicht nur um die Entwicklung der Alltagsontologie in Auseinandersetzung mit Quantorenvarianz, sondern auch um alltagsontologische Weiterentwicklung der Quantorenvarianz-Position. Das unterscheidet das Folgende von der alltagsontologischen Diskussion mit der Easy Ontology und mit der Streit-um-Worte-Partei. In Analogie zu den vorhergehenden Abschnitten können wir auch bei unserer einschlägigen Behandlung der Quantorenvarianz mit Übereinstimmungen beginnen. Danach folgt eine Grenzziehung zwischen Quantorenvarianz und Alltagsontologie, welche auf bestimmte (Extrem-)Varianten bzw. unerwünschte „Nebenwirkungen“ Bezug nimmt, um im Anschluss daran das strategische Ziel, siehe letzter Absatz, verfolgen zu können.

Eine erste wichtige Übereinstimmung ist die anti-idealistische Tendenz innerhalb der Quantorenvarianz-Position, bzw. deren Begründung, welche uneingeschränkt auf die Alltagsontologie zu übertragen ist. Das ist an dieser Stelle deshalb zu unterstreichen, weil ja beide, Quantorenvarianz und Alltagsontologie, zumindest auf den ersten Blick, mit bestimmten Verdachtsmomenten in Richtung Idealismus konfrontiert werden könnten. Bei der Quantorenvarianz ist das besonders naheliegend, weil sich führende Exponenten wie Putnam, wie gesehen, nicht explizit dagegen verwahren, um es vorsichtig zu formulieren. Für standardmäßige Theorien im Rahmen der Quantorenvarianz-Theorie, insbesondere Hirsch ist hier zu erwähnen, gilt jedoch, dass sich aus der These der variierenden Bedeutung des Begriffs „Existenz“ bzw. des logischen Zeichens des Existenzquantors keinesfalls die Abhängigkeit des Existierenden von Begriffen bzw. von Zeichen einer bestimmten normierten Notation ergibt (siehe Abschnitt 2.13). Dementsprechend kann man auch den (Kurz-)Schluss zurückweisen, dass sich aus der alltagsontologischen Annahme der Bezogenheit von ontologischen Schemata auf alltägliche Praxis, 
damit verbunden deren historische und auch kulturelle Varianz, die Abhängigkeit dessen, was ist, von dieser Praxis ergibt.

Eine weitere, sofort ins Auge springende Übereinstimmung der Quantorenvarianz-Position, v.a. in der Auslegung von Eli Hirsch, mit der Alltagsontologie ist der Ausgang von Überlegungen bei einem Bezug auf das, was Hirsch ,plain-English“ nennt. Im Abschnitt 2.13 haben wir ja gesehen, dass Hirsch bei aller Vielfalt möglicher Bedeutungen von „Existenz“ eine faktische und somit auch prioritäre annimmt. Das aber ist nicht „Existenz“ in irgendeiner Kunstsprache, auch nicht eine durch eingeschränkte methodische Perspektiven festgelegte Bedeutung des Existenzquantors. Der Ausgang des Verstehens von „Existenz“, somit die bevorzugte Verständnisweise von Existierendem, manifestiert sich nach Hirsch im alltäglichen Sprachgebrauch.

Das kann gleichlautend als Grundmaxime alltagsontologischer Theorienbildung übernommen werden. Alltäglicher Sprachgebrauch ist Alltagspraxis. Deren Existenzvoraussetzungen, nicht die einer speziellen, etwa methodisch limitierten einzelwissenschaftlichen Praxis ist der Ausgang und prioritäre Bezugspunkt einer alltagsontologischen Theorie von Existierendem. So wurde ja auch die Interpretationskompetenz von Grundstrukturen unserer alltäglichen Sprache als Kriterium für den Status einer These als alltagsontologischer angenommen.

Die Tragweite dieser Überschneidungen zwischen Alltagsontologie und Quantorenvarianz-Theorie, die an dieser Stelle nur festgehalten sein soll, wird uns gleich wieder, im Zusammenhang mit wechselseitiger Weiterentwicklung beider Zugänge zur Ontologie, beschäftigen. Zunächst soll zur Klärung des Verhältnisses von Alltagsontologie und Quantorenvarianz der Blick aber auch auf gewisse Unvereinbarkeiten gelenkt werden. Dies ist schon deshalb nötig, um die genannten Übereinstimmungen nicht zum Anlass einer vorschnellen Ineinssetzung von Alltagsontologie und Quantorenvarianz zu nehmen.

Bei den Differenzen soll zwischen solchen unterschieden werden, die auf bestimmte (Extrem-)Varianten der Quantorenvarianz-Position Bezug nehmen, und solchen, die auf unerwünschte „Nebenwirkungen“ von Hirschs Standardtheorie verweisen. Kommen wir zunächst zum ersten Gesichtspunkt, der sich als Absetzung von Ryles Annahme einer äquivoken Bedeutungsviel- 
zahl von „Existenz“, damit verbunden von einer Trivialisierung ontologischer Fragen (siehe 2.13), darlegen lässt. Führt man „Existenz“ über Voraussetzungen alltäglicher Praxis ein, kann man das Existierende, sprich das Vorausgesetzte, nicht in eine zusammenhangslose Pluralität aufgliedern. Der Bezug zur alltäglichen Praxis stellt nämlich eine Verbindung dar, die trotz unterschiedlicher Art, wie bestimmte Existierende in der Praxis präsupponiert werden, nicht zu einer Vielzahl, im Sinne von Ryles Äquivokation, von so eingeführten Entitäten führen kann. Nehmen wir an, wir erachteten sowohl diachron identische Dinge als auch Ereignisse, in die sie involviert sind, als Existenzvoraussetzungen alläglicher Praxis. So mag es durchaus sein, dass die Existenz von Dingen und die von Ereignissen auf unterschiedliche Weise in unserer alläglichen Praxis vorausgesetzt werden. Auch mag, wie an verschiedenen Orten angedeutet, die Annahme der Existenz von Ereignissen auf bestimmte Art von der der Dinge abhängen. Dennoch, führt man „Existenz“ über unterscheidbare Voraussetzungen alltäglicher Praxis ein, kann man das Existierende, z.B. Dinge und Ereignisse, nicht in eine zusammenhangslose kategoriale Vielheit aufgliedern. Dinge und Ereignisse sind angenommen als unterschiedliche Voraussetzungen bzgl. Existierendem derselben Praxis. Auch der bei Ryle mit der Äquivokation von „Existenz“ verbundenen Trivialisierung der Frage nach bestimmten Kategorien von Entitäten entgeht man. Die alltagsontologische Frage nach den Existenzvoraussetzungen alltäglicher Praxis lässt sich, gerade in Anwendung der drei immer wieder angesprochenen Kriterien Intuitivität, Entsprechung zu Grundstrukturen alltäglicher Sprache, Entwickelbarkeit, nicht trivial beantworten. Ob, um beim Beispiel zu bleiben, Dinge und Ereignisse, als Existenzvoraussetzungen alltäglicher Praxis, als Entitäten anzunehmen sind, hängt von einer Prüfung gemäß gegebener Kriteriologie ab. Diese mag in bestimmten, wie den unsrigen Fällen zu klaren Ergebnissen führen. Trivial ist eine solche Prüfung jedoch nicht.

Wir können damit zu unerwünschten „Nebenwirkungen“ von Hirschs Theorie innerhalb der Quantorenvarianz-Position kommen: seine Vorschläge, ontologische Dispute durch Verweis auf Quantorenvarianz aufzulösen und damit gegen eine hypertheoretische Ontologie im Sinne von ontological shallowness zu plädieren. Der Gegensatz zur Alltagsontologie ist dadurch klar zu machen, dass Letztere antritt, um ontologische Dispute zu lösen, nicht aufzu- 
lösen. Das lässt sich an sämtlichen Standardbeispielen darlegen. Paradigmatisch kann allerdings wiederum die endurer-perdurer-Debatte herangezogen werden; auch deshalb, weil sie bei Hirsch durch den Verweis auf Quantorenvarianz deflationiert wird. Zur Erinnerung: Dieser Streit wird durch die Einführung zweier Bedeutungen von „Existenz“ bzw. zweier verschiedener Quantoren, $\exists_{\mathrm{E}}$ und $\mathrm{G}_{\mathrm{P}}$, aufgelöst. Alltagsontologisch ist das nicht. Hier ist die Debatte zu führen, ob die Annahme von durch die Zeit identischen Entitäten oder, alternativ, deren Ersetzung durch Summen numerisch verschiedener raum-zeitlicher Teile besser geeignet ist, Existenzvoraussetzungen unserer alläglichen Praxis zu verstehen. Sie ist zu führen und nach den bekannten Kriterien zu entscheiden. In diesem Sinn ist die Alltagsontologie wohl eher hypertheoretical als shallow, wenn wir gezwungen wären, sie im Spektrum von Hirschs polemischen Attributen zu positionieren. Auch wenn alltagsontologische Theorienbildung auf Praxis bezogen ist, ist sie nicht pragmatisch, sondern hochtheoretisch vorgetragen. Auch wenn es uns nicht schwer, sondern leicht fällt, einen Zugang zu unserer alltäglichen Praxis zu finden, wird die systematische Reflexion auf ihre Existenzvoraussetzungen nicht shallow. Schließlich: Selbst wenn wir semantische Überlegungen, etwa über die Interpretation von Grundstrukturen unseres alltäglichen Sprechens, als Kriterium für Alltagsontologie annehmen, werden dadurch ontologische Probleme nicht zu sprachlichen bzw. rein semantischen.

Zumindest bei dem zuletzt genannten Gesichtspunkt fallen Überschneidungen mit den Demarkationslinien zwischen Alltagsontologie und Easy Ontology ins Auge. Auch die Easy Ontology, darin ähneln sich ihre „Nebenwirkungen“" mit jenen von Hirschs Ansatz der Quantorenvarianz, schreibt sich ja Einfachheit und somit Kampf gegen theoretische Schwere, verbunden mit einem begriffsanalytischen Zugang zur Ontologie, auf ihre Fahnen. Um Redundanzen in der Darlegung zu vermeiden, können wir deshalb an dieser Stelle auf die einschlägigen Ausführungen in den Passagen zur Easy Ontology verweisen, Stichwörter: Verteidigung der kognitiven Relevanz „,schwerer“ ontologischer Fragen, Zurückweisung der Einschränkung ontologischer Themen auf Existenzfragen u.a. Sie lassen sich unter der angesprochenen Rücksicht auch auf Hirsch anwenden. 
Das lässt uns zum dritten und hauptsächlichen Gesichtspunkt kommen: wechselseitige Weiterentwicklung von Alltagsontologie und Quantorenvarianz. Wir können damit beginnen, dass wir in der Alltagsontologie durchaus mit variierenden Bedeutungen von „Existenz“ operieren. Wie schon gesagt, unsere alltägliche Praxis ist vielfältig, gerade im Hinblick auf Voraussetzungen bzgl. Existierendem. Im Kern ist diese Praxis wohl auf etwas angewiesen, das auch im Zuge von Veränderung in der Zeit in einem strikten Sinn identisch bleibt. Dieser, wenn man so will, statische Zug unter den Existenzvoraussetzungen ist jedoch nicht alles. Auch Veränderung ist ein wesentliches Element dessen, was wir voraussetzen, wenn wir versuchen, unser alltägliches Leben im Hinblick auf Existierendes zu verstehen. Da erscheint es naheliegend, in jenem ontologischen Rahmen, in dem wir diese Voraussetzungen systematisch entwickeln, eine grundlegende Verschiedenheit anzunehmen, die sich auch in der Annahme unterschiedlicher Existenzweisen, eben in unterschiedlichen Bedeutungen von „Existenz“ widerspiegelt. Dieses alltagsontologische Verstehen von „Existenz“ ist also keineswegs auf das, was Hirsch, leicht missverständlich, auch „God's quantifier“ nennt, angewiesen. Das Gegenteil ist der Fall: Die Alltagsontologie operiert nicht mit Symbolen, die strikt oder dogmatisch auf eine einzig mögliche Bedeutung von „Existenz“ festlegen. Und zwar deshalb nicht, weil dies den Bezug zu den Voraussetzungen alltäglicher Praxis ausblendet, bzw. zu jener eben angesprochenen Vielfalt von Existenzweisen, die dabei ins Spiel kommen. Alltagsontologie, so könnte man es auf den Punkt bringen, ist gerade nicht auf ein univokes Verstehen von „Existenz“ festgelegt.

Diese Variationen in den Bedeutungen von „Existenz“ bzw. des Existenzquantors aber lassen sich vermitteln. Es gibt stabile Brücken zwischen diesen unterschiedlichen Bedeutungen, die, wie eben dargelegt, damit zu tun haben, dass sie sich auf verschiedene Weise aus den Voraussetzungen bzgl. Existenz einer Praxis ergeben. Alltagsontologie steht auch einer äquivoken Vielfalt von Existenz-Begriffen entgegen.

Unter der alltagsontologisch akzeptierten Vielfalt von Bedeutungen von „Existenz“ aber kann man eine privilegierte annehmen. Obwohl die explizite Begründung dafür Sache der Entfaltung einer kategorialen Ontologie ist, können wir hier jene Bedeutung als besonders geeigneten Kandidaten annehmen, 
welche der Existenzweise der immer wieder angesprochenen endurer entspricht. Dies gibt durchaus auch die Einschätzung Hirschs des Stellenwerts des endurer-Quantors, $\exists_{\mathrm{E}}$, im plain-English wieder. Dieser Quantor läuft jedenfalls über Entitäten, die in einem strikten Sinn diachron identisch, deshalb auch Träger von Eigenschaften und deren Änderungen sind. Dies wird unverzichtbar von unserer alltäglichen Praxis vorausgesetzt. Anders hat diese Praxis keinen Sinn. Die Annahme von Elementen im Wertebereich von $\exists_{\mathrm{E}}$ entspricht unseren Intuitionen und kann als Teil einer legitimen Interpretation von Grundstrukturen unseres alltäglichen Sprechens gelten.

Die besagten Eigenschaften und auch Änderungen, als Ereignisse kategorisiert, hängen aber in einer genau zu erläuternden Weise von ihren Trägern ab. Das legitimiert die Rede, dass Eigenschaften und Ereignisse Entitäten mit anderen Existenzweisen als endurer sind. Auch solche Priorisierungen bzw. Abhängigkeiten werden selbstverständlich in unserem alltäglichen Leben angenommen und in unserer Alltagssprache zum Ausdruck gebracht - etwa, wie gesehen, durch Priorisierung bzw. Abhängigkeit von sprachlichen Identifikationsmitteln.

Der springende Punkt an dieser Stelle ist, dass wir ausgehend von einer angenommenen eigentlichen Kernbedeutung von „Existenz“, das ist jene von $\mathrm{G}_{\mathrm{E}}$, bestimmte Differenzierungen vornehmen können. Die jeweiligen Bedeutungen dieser Differenzierungen, mögliche Beispiele sind Quantoren, die über Ereignisse bzw. über partikulare Eigenschaften laufen, ergeben sich aber aus dem vergleichenden Bezug zu der angenommenen Kernbedeutung. Daraus lassen sich auch ihre Verbindungen ersehen, sowohl untereinander als auch im Hinblick auf die angenommene eigentliche Bedeutung. Die verschiedenen Bedeutungen von „Existenz“ entsprechen Elementen eines Gefüges von Voraussetzungen bzgl. Existierenden, das sich, wie gesagt, als ontologische Interpretation der einen alltäglichen Praxis verstehen lässt. Das lässt den Ausblick auf ein analoges Verstehen von Existenz in der Alltagsontologie $\mathrm{zu}^{171}$

${ }^{171}$ In der aktuellen metaontologischen Debatte hat Kris McDaniel den Vorschlag eines analogen Verstehens von Existenz eingebracht, vgl. u.a. ders. 2009. McDaniel geht davon aus, dass beschränkte Quantoren (engl.: restricted quantifier), das sind solche, die über nichtleere, nicht-überlappende Bereiche bestimmter Entitäten laufen, grundlegende Quantorenbedeutungen ausmachen, der generisch unbeschränkte Quantor relativ dazu aber als abgeleitet 
Einen entscheidenden Schritt weitergehen können wir, wenn wir die Möglichkeit ins Auge fassen, alternative Sprechweisen zu entwickeln, im Sinne des von Hirsch ins Spiel gebrachten als ob. Zur Erinnerung: Hirsch zieht die Möglichkeit in Betracht, vom Standpunkt einer endurer-Sprache davon abweichende Sprechweisen, etwa eine perdurer-Sprache, anzunehmen. Wir können als EndurantistInnen so sprechen, als ob wir PerdurantistInnen wären, also auch so, dass wir den Aussagen in der perdurer-Sprache positive Wahrheitswerte zuschreiben. Angewandt auf die vorhin entwickelten Überlegungen bzgl. Alltagsontologie können wir annehmen, dass wir als AlltagsontologInnen, die mit Gründen die endurer-Redeweise für grundlegend halten, so reden könnten, als $o b$ nicht ein endurer-Quantor die eigentliche Bedeutung von „Existenz“ darstellte, sondern die Bedeutung eines sich an der Existenzweise von perdurer, das sind Summen numerisch verschiedener, raum-zeitlicher Teile, orientierenden Quantors $\Xi_{\mathrm{P}}$; bzw. die Bedeutung eines der Existenzweise von Tropen, das sind partikulare Qualitäten, die im Bündel alltägliche Dinge konstituierten, entsprechenden Quantors $\mathrm{G}_{\mathrm{T}}$.

Von der alltagsontologisch privilegierten Perspektive aus können wir so sprechen, als $o b$ die Alternative adäquat wäre. Dies kann so weit gehen, dass wir durchaus auch Sätzen der geschilderten alternativen Sprechweisen positive Wahrheitswerte zuschreiben. Das setzt voraus, dass wir in der Lage sind, dieses als $o b$ im Rahmen einer systematischen Analyse nachvollziehbar zu machen. Das kann z.B. so geschehen, dass wir vom alltagsontologischen Standpunkt aus, der ja eine Universaltheorie der Existenzvoraussetzungen aller Praktiken anzielt, auch Sprachen untersuchen und verstehen wollen, die den methodisch legitimen Einschränkungen einzelwissenschaftlicher Praktiken entsprechen. Dazu kann es erforderlich sein, so zu sprechen, als ob z.B. Modellierungen im Sinne eines vierdimensionalen Raum-Zeit-Systems, wie sie in der physikalischen Praxis der Theorienbildung erfolgreich angenommen werden, auch ontologisch adäquat wären. Das wiederum geht nicht,

aufzufassen ist. Es würde zu weit führen, das hier vorgelegte Verständnis der Analogie von Existenz mit jenem von McDaniel zu vergleichen. Ein Unterschied mag jedenfalls darin bestehen, dass McDaniel eine Mehrzahl fundamentaler Quantorenbedeutungen annimmt (u.a. ebd., 314), was ihn auch in die Nähe der Quantorenvarianz-Position bringt. Hier wird für die grundlegende Bedeutung des endurer-Quantors argumentiert. Ein generischer, unbeschränkter Existenzquantor spielt in der Alltagsontologie keine Rolle, auch keine abgeleitete. 
ohne so zu sprechen, als ob $\exists_{\mathrm{P}}$ die eigentliche Bedeutung von „Existenz“ wäre. Oder es mag angebracht sein, so zu reden, als ob quantentheoretische Modelle, in denen eigenschaftsartige Objekte mit vager Identität gesetzt sind, tatsächlich Entitäten entsprächen. Dann könnte man so sprechen, als ob eine Art Tropen-Quantor, $\exists_{\mathrm{T}}$, die Kernbedeutung von „Existenz“ darstellte.

Von unserer durch $\exists_{\mathrm{E}}$ geprägten Sprache können wir nun durchaus auch die alternativen Sprechweisen mit positiven Wahrheitswerten versehen; wenn wir ja nicht vergessen, dass es sich um theoretisch legitime mögliche Abweichungen von der privilegierten Quantorenbedeutung handelt, die faktisch, wie auch Hirsch in vergleichbarem Zusammenhang betont, keine Verpflichtung auf Entitäten mit sich bringt.

Bei Hirsch finden wir die Annahme, dass die Frage, welche Sprachform nun die privilegierte, welche aber zu den abgeleiteten zu zählen ist, letztlich als Sache der Pragmatik abgetan werden kann. Spätestens hier haben wir als AlltagsontologInnen die Gesellschaft Hirschs, die uns ja durchaus auch gut tut, zu verlassen. Die Entscheidung über die privilegierte Quantorenverwendung bzw. über deren Als-ob-Ableitungen ist eine hochtheoretische, folglich ontologisch schwere. Hier muss für einen Standpunkt, z.B. die Priorität von $\mathrm{G}_{\mathrm{E}}$, argumentiert werden. Es ist ein Streit zu führen mit jenen, die annehmen, dass nicht die Existenzvoraussetzungen alltäglicher Praxis prioritär sind im Hinblick auf einen universalen ontologischen Rahmen, sondern die Existenzvoraussetzungen der Praxis einer Einzelwissenschaft. Letztere werden, wie angedeutet, $\exists_{\mathrm{P}}$ oder $\exists_{\mathrm{T}}$ den Vorrang geben, $\exists_{\mathrm{E}}$ aber als „unwissenschaftlich“ abtun, bzw. nur im Kontext uneigentlicher Als-ob-Redeweise durchgehen lassen. Führen wir diesen Streit, entwickeln wir die Theorie der Quantorenvarianz alltagsontologisch weiter. ${ }^{172}$

Der Streit selbst geht über die Auseinandersetzung mit Quantorenvarianz als Position innerhalb des Deflationierungsprogramms hinaus. Er ist vielmehr zu führen im Rahmen der alltagsontologischen Diskussion mit szientistisch-

\footnotetext{
${ }^{172}$ Dieser Streit, um das am Rande einzustreuen, ist natürlich keiner ,um Worte“ im Sinne einer Stoßrichtung der Deflationierungskritik an der Ontologie (vgl. 2.11, 3.21). Keinesfalls kann ein/e VertreterIn der Priorität des endurer-Quantors einen Sprachgebrauch akzeptieren, im Sinne von Kriterium (i) eines Streits um Worte, in dem ein perdurer-Quantor oder ein Tropen-Quantor diese priviligierte Position einnimmt, die endurer-Redeweise aber lediglich im Sinne eines ,als ob“ legitim wäre.
} 
revisionären Einstellungen, wie sie typisch sind für das Naturalisierungsprogramm. Das soll im Folgenden geschehen.

\subsection{Alltagsontologie und Naturalisierung}

Bevor wir an dieser Stelle in medias res gehen, soll aus gegebenem Anlass die Eigenart der nunmehr zu führenden Kontroverse hervorgehoben werden (3.221). Dann soll es, in Analogie zu den vorhergehenden Abschnitten, auch um bestimmte Übereinstimmungen mit Detailthesen aus naturalistischen Positionen gehen (3.222), bevor die Argumentation gegen den Naturalismus als ontologisches Programm aus Sicht der Alltagsontologie vorgetragen wird (3.223).

\subsection{Vorbemerkungen zu einer Kontroverse}

In der nunmehr zu führenden Kontroverse geht es um die Begründung einer Priorisierung, und zwar - alltagsontologisch gesprochen - der Existenzvoraussetzungen unserer alltäglichen Praxis vor jenen einer Einzelwissenschaft. Diese Begründung hat in kritischer Auseinandersetzung $\mathrm{zu}$ erfolgen mit VertreterInnen der Gegenposition, sprich des Naturalisierungsprogramms. Diese Begründung wurde bereits in vorhergehenden Abschnitten postuliert; z.B. am Ende des Abschnitts 3.13 „Ontologie als Alltagsontologie“, wo davon die Rede ist, dass das Betreiben von Alltagsontologie auf einer $\mathrm{Be}$ wertung zu beruhen hat. Diese Bewertung besteht darin, dass Alltagsontologie gegenüber revisionärer Ontologie der Vorrang zu geben ist. Wenn, wie angenommen, NaturalistInnen in der Ontologie den Standpunkt der Revision vertreten, muss eine entsprechende argumentative Auseinandersetzung erfolgen, um Alltagsontologie zu legitimieren. Diese Auseinandersetzung sollte dann auch die gerade angesprochene Priorisierungsfrage bzgl. des Verstehens von „Existenz“ entscheiden. Dahingehend, dass der endurer-Quantor, $\exists_{\mathrm{E}}$, grundlegend ist, $\exists_{\mathrm{P}}$ bzw. $\exists_{\mathrm{T}}$ hingegen nur im Sinne einer Als-ob-Redeweise adäquat sind. Es geht hier also nicht nur um die Rechtfertigung einer sich an deskriptiver Metaphysik orientierenden Alltagsontologie im Allgemeinen, sondern auch um die anvisierte alltagsontologische Weiterentwicklung der Quantorenvarianz. 
Eine Eigenart der Auseinandersetzung zwischen Alltagsontologie und Naturalismus besteht nun allerdings darin, dass sie nicht als binnenontologischer Disput geführt werden kann, zumindest nicht ausschließlich. Und zwar deshalb nicht, weil von beiden Parteien ein bestimmtes Verstehen von Naturwissenschaften bzw. naturwissenschaftlicher Praxis vorausgesetzt wird. Dieses Verstehen betrifft die Frage, inwiefern Naturwissenschaften Existenzaussagen im Sinne auch der Ontologie tätigen, bzw. ob die naturwissenschaftliche Praxis als solche überhaupt Voraussetzungen bzgl. Existierendem macht. NaturalistInnen müssen beides bejahen, und können (nur) darauf aufbauend sogar die besondere Autorität von Naturwissenschaften, insbesondere der (Quanten-)Physik, im Hinblick auf Existenz annehmen, bzw. eben den Vorrang der Existenzvoraussetzungen physikalischer Praxis im Hinblick auf eine Universaltheorie von Existenzvoraussetzungen. Ob und inwieweit aber Naturwissenschaften tatsächlich von Existenz bzw. Existierendem oder Entitäten handeln, ist etwas, das OntologInnen alleine gar nicht entscheiden können. Das muss im Grunde mit Blick auf die naturwissenschaftliche Praxis bedacht werden.

Deshalb, und damit komme ich zu meinem Punkt, soll die gegen den Naturalismus gerichtete alltagsontologische Begründung der Priorität der Existenzvoraussetzungen alltäglicher Praxis bedingt vorgetragen werden; und zwar, um es vorwegzunehmen, so, dass Alltagsontologie dann (und nur dann) als Universaltheorie alles Existierenden aufgefasst werden kann, wenn es den Naturwissenschaften, allen voran der Physik gerade nicht um Existenz geht; bzw. wenn zutrifft, dass, was auch immer in der einzelwissenschaftlichen Praxis der Physik vorausgesetzt wird, von der Physik gerade nicht als Existenzvoraussetzung verstanden wird; wenn somit die Interpretation von physikalischen Forschungsergebnissen im Hinblick auf Existenz autonome Sache der Ontologie ist.

Ist das ein Ausweichmanöver, das die Konfrontation mit den apodiktischen Behauptungen des Naturalismus fürchtet? Nein! Denn letztlich beruht auch naturalistische Ontologie auf einer Voraussetzung, die in einem Konditionalsatz zum Ausdruck gebracht werden kann, dessen Vordersatz ebenfalls nicht nur eine hinreichende, sondern auch notwendige Bedingung enthält, und der im Folgenden - cum grano salis - ,naturalistischer Konditional“ ge- 
nannt werden soll: Wenn Naturwissenschaften die eigentlichen Autoritäten in Existenzfragen sind, dann (und nur dann) hat man die Ontologie ausgehend von den Ergebnissen physikalischer Forschung als a posteriorisches Projekt aufzuziehen. Nur wird von NaturalistInnen die Bedingung im Vordersatz für gewöhnlich nicht als solche explizit gemacht. Aus einem Konditional wird so, ungerechtfertigterweise (!), eine affirmative Aussage bzgl. des Verhältnisses zwischen Physik und Ontologie: Die Ontologie ist a posteriori.

Begeben wir uns mit bedingten Begründungen aber nicht doch wieder in eine Abhängigkeit von Naturwissenschaften? In gewisser Weise schon; allerdings in keine einseitige, sondern in eine dialogische. Als AlltagsontologInnen bieten wir uns Einzelwissenschaften als ExpertInnen in Sachen Existenz an und sind darauf angewiesen abzuwarten, wie mit diesem Angebot verfahren wird. Jedenfalls ernennen wir Einzelwissenschaften nicht, gegen deren Intention, zu Auskunftsgeber über Entitäten. Dialog bringt natürlich auch Risiken mit sich. Diese sollte man allerdings im Sinne der Vermeidung dogmatischer bzw. prämissenverschleiernder Theorienbildung ruhig eingehen.

Trotz dieser gegenüber dem Naturalismus bewusst konfliktheischenden Hinführung soll im Folgenden überlegt werden, ob es, wenn schon nicht mit dem Programm des Naturalismus, so doch mit einzelnen Elementen naturalistischer Positionen Überschneidungen bzw. Übereinstimmungen gibt, die wir für den Fortgang von Alltagsontologie nützen können.

\subsection{Was man als Alltagsontologe von NaturalistInnen lernen kann}

Eine allgemeine Sympathiebekundung gegenüber VertreterInnen des Naturalisierungsprogramms kann beim Anliegen der Naturalisten der Gründungsgeneration der Analytischen Philosophie ansetzen, hohe begrifflich-argumentative Standards im philosophischen Denken zu etablieren (siehe Abschnitt 2.21). Dem kann man sich auch als Alltagsontologe nicht verschließen, gerade wenn es darum geht, durch unklare Sprachverwendung erzeugte philosophische Probleme zu vermeiden. Konkreter mag ein weiterer Hinweis auf Carnap ausfallen. Und zwar der, dass Carnap seine szientistische Weltanschauung explizit als Voraussetzung seines Naturalismus bzw. Physikalismus nennt. Als ein tragendes Element dieser Weltanschauung können wir es erachten, dass die Naturwissenschaften, allen voran die Physik, die Autoritäten 
in Sachen Welt- bzw. Wirklichkeitserklärung sind. ${ }^{173}$ Damit wird ihnen auch eine privilegierte Stellung bzgl. der Klärung von Existenzfragen zugebilligt. Wenn dem so wäre, dann (und nur dann) folgten daraus die Grundannahmen naturalistischer Ontologie. Wir dürfen also annehmen, dass bei Carnap der in den Vorbemerkungen erwähnte ,naturalistische Konditional“" durchaus transparent gemacht wird. Provokanterweise ließe sich die Mutmaßung anstellen, dass Carnap wohl nichts dagegen gehabt hätte einzuräumen, dass er widerlegt wäre, wenn sich die Bedingung im Vordersatz dieses Konditionals als unerfüllt herausstellt. Damit hätte er nämlich nie und nimmer gerechnet.

Angebracht ist es auch, unter der nunmehr zur Debatte stehenden Rücksicht auf Quine Bezug zu nehmen. Zunächst kann eingestanden werden, dass Quines formaler Apparat richtungsweisend für jedwede analytische Ontologie ist; wobei ,richtungsweisend“ durchaus die Möglichkeit kritischer Distanzierung oder zumindest kreativer Weiterentwicklung mit einschließt. Als Bezugspunkt ist Quine jedenfalls unverzichtbar. In diesem Sinne sei auf einige Implikationen der Anwendung von Quines formalem Apparat hingewiesen, welche auch für die Alltagsontologie begrüßenswerte Resultate mit sich bringen. Da ist zunächst die Festlegung darauf, dass es sich bei Existenz, im alltagsontologischen Verstehen, nicht um eine Eigenschaft handelt, ${ }^{174}$ zumindest nicht eine solche erster Stufe (Frege), oder „von dem Dinge selbst“ (Kant). ${ }^{175}$ „Existenz“ ist, logisch betrachtet, ein Prädikat, das von Begriffen ausgesagt wird (Kant und Frege), um von ihnen zu behaupten, dass ihre Extension nicht gleich 0 ist. ${ }^{176}$ Es würde hier zu weit führen, eine alltagsontologische Entfaltung von Eigenschaften im Allgemeinen und von Existenzprädikationen im Besonderen einzuschieben. ${ }^{177}$ An dieser Stelle sei jedoch festgehalten, dass der diesbezügliche Status von „Existenz“ sowohl bei Quine als auch in einer Alltagsontologie ein wichtiges metaontologisches Theoriestück ist. Dasselbe gilt von der Ineinssetzung von „Sein“ und „Existenz“ bzw. der

\footnotetext{
173 Dies macht Carnap in seiner Autobiographie Mein Weg in die Philosophie besonders deutlich; siehe Carnap 1993, u.a. 11.

174 ... wie das in der Gegenwartsliteratur v.a. Geach vertreten hat, siehe ders. 1968.

175 Vgl. Frege 1884, Kant 1968, 72.

${ }^{176}$ Vgl. dazu u.a. Röd 1989.

177 Dazu sei, insbesondere im Hinblick auf die Existenz-Thematik, auf Kanzian 2016, 183189 , verwiesen.
} 
Annahme, dass kein über Existierendes hinausgehender Seinsbereich anzunehmen ist, wie das in aktuellen Metaontologien v.a. meinongianischer Prägung vertreten wird. Dass eine solche Aufspaltung zwischen Sein und Existenz in einer sich an Quine orientierenden Theorie unannehmbar ist, hat in letzter Zeit Peter van Inwagen klar dargelegt. ${ }^{178}$ Wir können uns hier auf ihn berufen und auch diese These als Desiderat einer Alltagsontologie kennzeichnen, ohne das an dieser Stelle ausführen zu können.

Hier soll vielmehr auf einen weiteren Aspekt von Quines formalem Apparat hingewiesen werden, an den wir in der Alltagsontologie gewinnbringend anknüpfen können: Im Kern eines solchen Anknüpfens steht das Anliegen, den Aufweis der Voraussetzungen einer bestimmten Praxis bzgl. Existenz in Zusammenhang mit Quines ontologischen Verpflichtungen (engl.: ontological commitments) bestimmter Sprechweisen zu verstehen. Ansetzen kann man dabei durchaus bei Quine selbst, der ja die physikalische Sprache als jene Ausdrucksform auffasst, in der die Voraussetzungen physikalischer Praxis bzgl. Existenz deutlich werden, wenn wir diese Sprache in der kanonischen Notation der Prädikatenlogik erster Stufe darlegen. Diesen Ansatz aber kann man verallgemeinern, dahingehend, dass in entsprechend „kanonisch“ formalisierten Sprechweisen die ontologischen Voraussetzungen einer bestimmten Praxis bzgl. Existenz zu eruieren sind. Somit ist es legitim anzunehmen, dass sich in der Alltagssprache, prädikatenlogisch notiert, die Voraussetzungen unserer alltäglichen Praxis bzgl. Existenz zeigen. Auf diese Weise können wir wieder unseren endurer-Quantor ins Spiel bringen, der ja jene Bedeutung von Existenz paradigmatisch repräsentiert, welche die ontologischen Verpflichtungen unserer alltäglichen Praxis, ausgedrückt in der Alltagssprache, transportiert. So gesehen verpflichten wir uns, ganz im Sinne Quines, in unserer alltäglichen Praxis primär auf die Elemente des Wertebereichs von $\mathrm{B}_{\mathrm{E}}$, das sind synchron und diachron identische Entitäten, die Träger von Eigenschaften und deren Änderungen sein können. Damit klären wir, wie eben in Aussicht gestellt, mit Quines formalem Apparat die Voraussetzungen bzgl. Existierendem in unserer alltäglichen Praxis. Um einen gröBeren Bogen zu schlagen: Wir benötigen Quines Verstehen der ontologischen Verpflichtung durch die Verwendung von B, um von Freges Nicht-0-Quantor

${ }^{178}$ V.a. in van Inwagen 1998, Thesis 2.: Being is the Same as Existence, 235f. 
über Hirschs pragmatischer Priorisierung von $\mathrm{B}_{\mathrm{E}} \mathrm{zu}$ einer alltagsontologischen Perspektive auf den endurer-Quantor als grundlegender Verständnisweise von Existenz zu kommen.

Mit diesem Hinweis können wir wieder auf eine inhaltliche These zu sprechen kommen, die im Kern von Quines ontologischer Theorienbildung steht. Wie bereits unter 2.22 ausgeführt, erachtet Quine die ontologischen Verpflichtungen durch Aussagen in naturwissenschaftlichen Theorien als Setzungen (engl.: posits), die gleichsam als „convenient intermediaries“ zwischen begrifflichen Schemata und empirischen Daten fungieren. Das im besagten Abschnitt aufgegriffene Beispiel der Setzung von Molekülen macht deutlich, dass es sich dabei um modellhafte Interpretationen von experimentellen Befunden handelt, die dazu dienen sollen, empirischer Erfahrung eine möglichst gute, sprich nach Maßgabe der Methodik der Physik bzw. der Chemie praktikable Form zu geben. Es sei an dieser Stelle festgehalten, dass diese Auffassung von Setzungen bemerkenswerterweise richtungsweisend ist für das alltagsontologische Verstehen von Ergebnissen naturwissenschaftlicher Praxis. Dies wird uns im Kontext einer alltagsontologischen Kritik des Naturalismus noch ausführlich beschäftigen. Bereits hier kann vermerkt werden, dass der in Quines Auffassung von Setzungen zum Ausdruck kommende Anti-Dogmatismus ebenfalls als alltagsontologie-affin zu bezeichnen ist.

Carnap und Quine sind freilich nicht die einzigen Naturalisten, welche Thesen entwickelt haben, deren Berücksichtigung für eine Alltagsontologie maßgeblich ist. Auch bei Thomas Hofweber finden wir wichtige einschlägige Ansatzpunkte. In erster Linie sei in diesem Zusammenhang seine differenzierende Untersuchung der Verwendung von Quantoren erwähnt (siehe 2.22). Hofweber unterscheidet ja, wie gesehen, zwischen einer internen oder inferential role reading Verwendung von Quantoren und einer externen, die im Sinne eines domain conditions reading verstanden werden muss. Wir tun gut daran, eine Entsprechung dieser Distinktion in der Alltagsontologie zu etablieren. Nicht jede Verwendung von „Es gibt ..."-Partikeln in der Alltagssprache artikuliert nämlich eine Existenzvoraussetzung alltäglicher Praxis. Die Annahme des Gegenteils würde eine in der Tat naive Ontologisierung von Sprechweisen begünstigen, die in verschiedenen Kontexten bereits als problematisch herausgestellt wurde; gerade unter der Rücksicht, dass sich die Ontologie 
damit als Rahmen- oder Grundlagendisziplin über Existenz, auch in anderen Wissensbereichen, desavouieren würde. Da Alltagsontologie diesen, der Ontologie eigenen Anspruch erhebt, müssen wir in der Lage sein zu unterscheiden zwischen (im Sinne Hofwebers) internen Verwendungen von als Quantoren deutbaren „Es gibt..."-Partikeln und solchen, durch die tatsächlich Existenzvoraussetzungen transportiert werden.

Eine Möglichkeit, diese Distinktion alltagsontologisch zu etablieren, besteht wieder im Rekurs auf die Unterscheidung zwischen eigentlichen Redeweisen und Als-ob-Wendungen, die wir bereits in der Weiterentwicklung der Quantorenvarianz kennengelernt haben. Im Kern eigentlich ontologischer Redeweisen stehen nach alltagsontologischer Überzeugung solche, deren Existenzbehauptungen durch den endurer-Quantor zum Ausdruck gebracht werden. Durch diesen wird eine Domäne von Entitäten, noch dazu die primäre, festgelegt, durchaus im Sinne von Hofwebers domain conditions reading. Mit Hofweber könnte man diese Quantorenverwendung auch als ,paradigmatisch extern" bezeichnen. Das schließt nicht aus, dass nicht auch noch andere, analoge Existenzweisen anzunehmen sind, die durch Quantoren zum Ausdruck gebracht werden, deren Bedeutung in Zusammenhang mit dem endurer-Quantor zu entwickeln ist. Auch dies war bereits Thema in jenem Abschnitt, in dem Alltagsontologie und Quantorenvarianz als wechselseitige Bezugspositionen dargestellt wurden. Von den externen (Hofweber) Quantoren können wir nun solche „Es gibt..."-Redeweisen unterscheiden, die wir im Zuge von Als-ob-Abwandlungen der eigentlich ontologischen Redeweisen kennengelernt haben. Als Kandidaten können hier theoretische Setzungen naturwissenschaftlicher Theorien ebenso gehandelt werden, wie Reden über fiktive Gestalten, möglicherweise auch jene über mathematische Entitäten, um nur einige Beispiele zu nennen. Darin zeigt sich auch das Spektrum möglicher uneigentlicher oder interner (Hofweber) Quantorenbedeutungen, die im Kontext der Ausführung einer ontologischen Theorie sowohl untereinander, als auch im Hinblick auf ihren Bezug auf eigentliche zu entwickeln sind.

Damit kommen wir zu einem Aspekt, in dem sich ein wesentlicher Unterschied zwischen Hofwebers „bescheidener“ Ontologie (engl.: modest ontology) und unserer Alltagsontologie zeigt. Während Erstere die Unterscheidung zwischen den verschiedenen Interpretationen von Quantoren letztlich der 
Linguistik bzw. den Naturwissenschaften überlässt, bleibt dies - alltagsontologisch gesehen - der Entfaltung höchst unbescheidener ontologischer Theorienbildung vorbehalten. Was tatsächlich zu den Existenzvoraussetzungen alläglicher Praxis zu zählen ist, was hingegen im weiten Feld uneigentlicher Redeweisen bleibt, muss ontologisch begründet werden. Diese Bemerkung ist auch geeignet, den Duktus der Kontroverse mit dem Naturalisierungsprogramm aufzugreifen, was im Sinne der Einleitung zu diesem Abschnitt 3.22 umgehend in Angriff genommen werden soll.

\subsection{Warum das Naturalisierungsprogramm falsch liegt}

Aus den Ausführungen in vorhergehenden Abschnitten sollte bereits deutlich geworden sein, dass es sich beim Naturalismus um ein Programm handelt, das zu innerer Differenzierung durchaus in der Lage ist. So gibt es eine Vielzahl von Positionen quer durch die philosophischen Disziplinen, die in unterschiedlicher Stärke und mit unterschiedlichem Erklärungsziel spezifisch naturalistische Ansichten propagieren. Es kann hier nicht angezielt werden, diese Vielfalt darzustellen. Will man den Naturalismus kritisieren, ist das auch gar nicht erforderlich. Allerdings muss man klar machen, wie hier geschehen, dass es eben um den Naturalismus ausschließlich in der Ontologie geht. Außerdem hat man Kernthesen zu identifizieren, von denen man annimmt, dass sie naturalistische Ontologien als solche charakterisieren. Die anvisierte Kritik wird dann im Versuch bestehen, diese Kernthesen in Frage zu stellen.

Kandidaten für die Formulierung solcher Kernthesen haben wir ja bereits kennengelernt. Damit man eine ontologische Position dem Naturalismus zurechnen kann, muss diese jedenfalls die Ontologie als aposteriorisches Projekt auffassen: Ausgehend von naturwissenschaftlichen Ergebnissen erarbeitet die Ontologie Theorien über die Grundstrukturen der Wirklichkeit. Dabei kann man sich auf Carnap berufen, der diese als Ausdruck der Annahme einer universalen Welterklärungskompetenz der Naturwissenschaften entwickelt. $^{179}$ Auch Hofweber kann mit seiner prägnanten Formulierung „If anyone finds entities, it's the sciences $[\ldots]^{\star 180}$ als paradigmatischer Naturalist

${ }^{179}$ Einschlägige Belege finden sich in 2.22 bzw. 3.222.

${ }^{180}$ Hofweber 2009, 287. 
fungieren, ebenso wie Simons mit seiner bereits zitierten Bekundung: „Investigating the nature of the world and our relationship to it $[\ldots]$ is $[\ldots]$ a task $[\ldots]$ of a science revisable in the light of increasing knowledge about the world and ourselves [...]." ${ }^{181}$ Auf den Punkt bringen könnte man die den ontologischen Naturalismus charakterisierende Kernthese auch mit Jonathan Lowe: ,[...] it is these [empirical] sciences, if anything, that can tell us about the fundamental structure of reality." 182 Empirische Wissenschaften sind unsere Lehrmeister in Sachen Existenz.

Im Sinne der Bemerkungen in 3.221 kann man den naturalistischen Aposteriorismus allerdings auf eine Voraussetzung beziehen: Vorausgesetzt, dass empirische Wissenschaften, wie beispielsweise die Physik, die eigentlichen Autoritäten in Existenzfragen sind, dann (,und nur dann“ wird im gleich Folgenden aus Darstellungsgründen weggelassen) hat man die Ontologie ausgehend von den Ergebnissen der physikalischen Forschung als a posteriorisches Projekt aufzuziehen. Dann hat Hofweber Recht, dass seine externen Existenzfragen von den empirischen Wissenschaften, nicht von der Ontologie entschieden werden. Dann liegt Simons richtig, wenn er in eben erwähntem Sinne die oben zitierte Behauptung ergänzt durch „Investigating the nature of the world and our relationship to it is not a task for a priori metaphysics but of a science revisable in the light of increasing knowledge about the world and ourselves, a posteriori but still with a metaphysical framework of maximal generality at any stage". ${ }^{183}$ Dann ist auch Sider zuzustimmen, wenn er die Fachsprache der Physik als das wahre Ontologesisch (engl.: ontologese) bezeichnet. Dann mag schließlich auch die Behauptung gelten, dass sich ausgehend von den Voraussetzungen bzgl. Existenz einer speziellen Praxis, nämlich jenen der Physik, der Rahmen für eine Universaltheorie bzgl. Existenzvoraussetzungen aller Praxis darlegen lässt.

Wie bereits angedeutet, ist aber der springende Punkt, dass die im Vordersatz des „naturalistischen Konditionals“ angeführte Voraussetzung von NaturalistInnen für gewöhnlich nicht als solche explizit gemacht wird. Ihre Geltung wird stillschweigend angenommen, positiv interpretiert als Voraus-

\footnotetext{
${ }^{181}$ Simons 1998, 251.

182 Lowe 1998, 4.

${ }^{183}$ Simons 1998, 251.
} 
setzung weiterer Theorienbildung. So wird aus besagtem Konditional die ebenfalls bereits angeführte assertorische Behauptung, in kürzest möglicher Formulierung: Die Ontologie ist a posteriori.

Die hier versuchte Kritik am Naturalismus besteht nun darin, die naturalistische Assertion auf einen Konditional zurückzuführen, die Erfüllung der Bedingung im Vordersatz in Frage zu stellen und schließlich durch eine andere Bedingung zu ersetzen: Wenn es den Naturwissenschaften, v.a. der Physik, gerade nicht um Existenz geht, somit die Interpretation von physikalischen Forschungsergebnissen bzgl. Existenz autonome Sache der Ontologie ist, dann (,und nur dann“ lassen wir auch im ,,alltagsontologischen Konditional“ weg, wenn formale Akribie Lesbarkeit gefährdet) können die Existenzvoraussetzungen alltäglicher Praxis als Universaltheorie alles Existierenden aufgefasst werden. Nun soll es darum gehen, Gründe für die Ersetzung des naturalistischen Vordersatzes, sowie Argumente für die faktische Plausibilität des alltagsontologischen Folgesatzes anzugeben, wobei bei Letzterem in besonderer Weise die alltagsontologische Interpretation von Ergebnissen naturwissenschaftlicher Forschung im Hinblick auf Existenz maßgeblich ist.

\section{Naturwissenschaftliche Praxis als Modellbildung}

Die Darstellung, warum Naturwissenschaften keine Autoritäten in Existenzfragen sind, dass also die Bedingung im naturalistischen Konditional gerade nicht erfüllt ist, soll anhand von Überlegungen bzgl. der naturwissenschaftlichen Praxis geschehen. Diese Überlegungen seien als hypothetische Annahmen formuliert, deren Plausibilität durch Zeugen in der Literatur erhöht werden soll. $^{184}$

Die einschlägige Arbeitshypothese soll sein, dass naturwissenschaftliche, z.B. physikalische Praxis im Wesentlichen darin besteht, empirische Befunde zu gewinnen und nach Maßgabe ihrer Forschungsperspektive methodisch zu interpretieren; ferner, dass ein wesentlicher Aspekt dieser Interpretationsarbeit in der Anwendung von Modellen besteht. Der leitende Verdacht be-

${ }^{184}$ Bei diesen Überlegungen orientiere ich mich weitgehend an Ausführungen in Kanzian 2016, Abschnitt I - 2.2.2: Die Naturwissenschaften weisen in der Mikrowelt die Basisentitäten der Makrowelt auf - eine fundamentale Kritik. Hier werden diese Thesen in den Kontext Alltagsontologie gestellt. Die „Zeugenaussagen“ sind ebenfalls neu, bzw. aus anderen, noch anzugebenden Quellen gewonnen. 
steht darin, dass naturalistische OntologInnen den Fehler begehen, diese Modelle zu Entitäten zu stilisieren oder zu hypostasieren. Dieser Verdacht soll anhand der naturwissenschaftlichen Frage nach Atomen anfänglich illustriert und unter Verweis auf Prozesstheorien in Physik und Ontologie weiter erläutert werden.

Ohne den Anspruch zu erheben, Detailkenntnisse physikalischer Atomtheorien hier einbringen zu können, scheint doch eines klar zu sein: NaturwissenschaftlerInnen kommen zur Rede von Atomen nicht durch eine Abbildung von Gegenständen. Bei der Suche nach Atomen blickt niemand in ein Mikroskop, schießt im Erfolgsfall ein Foto und lässt dieses dann in einer Fachzeitschrift publizieren. Die Rede von Atomen hat ihren Ursprung in einer Deutung oder Interpretation von bestimmten empirischen Daten. Offensichtlich werden bei dieser Interpretation Modelle in Anschlag gebracht. Zur Illustration seien zwei, für unseren Kontext besonders markante, erwähnt. Das erste ist das Rosinenkuchen- oder Plum-pudding-Modell für Atome, entwickelt von Thomson (1903): Es nimmt seinen Ausgang von alltäglichen Zusammenhängen und versucht bestimmte experimentelle Daten zu deuten; etwa jene, die auf eine gleichmäßige Verteilung der positiven Ladung in der angenommenen Masse eines Atoms hindeuten, bei einzeln sich bewegenden negativ geladenen Teilchen. Das wenig später (1909) kolportierte Planetenmodell von Rutherford nimmt hingegen seinen Ausgangskontext aus der Astronomie. Nach diesem Modell bewegen sich die Elektronen ähnlich wie Planeten um einen Kern aus Protonen und Neutronen. Es kommt so mit bestimmten empirisch aufgewiesenen Daten, wie der Rutherford-Streuung, besser zurecht.

Bei späteren Modellen, etwa dem Schalen- bzw. dem Orbitalmodell, spielen hingegen mathematische Ausgangskontexte, etwa der Stochastik, eine entscheidende Rolle. Das kann hier ausgeklammert bleiben. Worum es geht, ist, dass die naturwissenschaftliche Rede von Atomen darauf beruht, dass ausgehend von bestimmten Ausgangskontexten modellhafte Interpretationen von experimentell gesicherten empirischen Befunden erfolgen. Halten wir jedenfalls fest: Thomson behauptet nicht, dass seine Theorie Rosinenkuchen an der Mikrobasis der materiellen Wirklichkeit abbildet; Rutherford nicht, dass es Elektronen gibt, die man wie Planeten fotografieren könnte, wenn man nur 
ein hinreichend leistungsfähiges Mikroskop zur Verfügung hätte. Allgemeiner gesprochen: Physikalischen Theorien geht es nicht um den Aufweis von Objekten und Gegenständen, schon gar nicht um Behauptungen bzgl. deren Existenz in einem ontologisch relevanten Sinn.

Heute gibt es im Rahmen des Naturalisierungsprogramms eine Vielzahl sogenannter ,atomistischer Ontologien“, welche nicht weiter analysierbare Entitäten (engl.: simples) als Grundelemente der Wirklichkeit annehmen. Aus diesen wird dann die alltägliche Welt rekonstruiert. Wenngleich es Quellen gibt, die das nahezulegen scheinen, ${ }^{185}$ soll nicht behauptet werden, dass sich diese an den eben dargestellten Modellbildungen orientieren. Das wird schon daran klar, dass die besagten physikalischen Atomtheorien gerade nicht mit simples operieren. Die Grundidee einer korpuskulären Grundstruktur der Wirklichkeit meinen naturalistische AtomistInnen aber dennoch aus naturwissenschaftlichen Theorien entnehmen zu können. Unter dieser Rücksicht lässt sich der angedeutete Leitverdacht auf sie beziehen: Naturalistische OntologInnen, nicht NaturwissenschaftlerInnen (!), ontologisieren Modelle zu Gegenständen oder Objekten, deren Aufweis als empirisch gesichertes Ergebnis der Naturwissenschaften gilt. Dann werden, im Bemühen um eine ,wissenschaftliche" Ontologie, aus diesen (vermeintlichen) Objekten ontologische Grundelemente gemacht: Entitäten, Existierendes. Modelle werden, um es kurz zu sagen, hypostasiert. ${ }^{186}$

So gut sich die Atomtheorie zur Illustration naturwissenschaftlichen Umgangs mit Modellen eignet, der Generalverdacht der Hypostasierung solcher Modelle lässt sich bei sogenannten Prozessontologien vermutlich noch besser

\footnotetext{
${ }^{185}$ U.a. Searle 1997, 16: „Für den gebildeten Menschen unserer Zeit ist es unabdingbar, dass er über zwei Theorien unterrichtet ist: die Atomtheorie der Materie und die Evolutionstheorie der Biologie“. Deshalb leitet sich, so Searle, ,zweifellos der größte Teil der Metaphysik aus der Physik her".

${ }^{186}$ Bzgl. des Verdachts der Hypostasierung im Hinblick auf Atommodelle finden wir bemerkenswerterweise in Wittgensteins Vorlesungen zur Ästhetik (hier: Wittgenstein 2005) Unterstützung; u.a. in folgender Notiz: „Du betrachtest das Atommodell für selbstverständlich. Was bedeutet das? Wir sind so sehr an das Bild gewöhnt, als hätten wir selbst alle Atome gesehen“ (ebd., 31). Und in einer Fußnote (Nummer 2) dazu: „Wir nehmen das Atommodell als selbstverständlich hin, was eine merkwürdige Sache ist". NaturalistInnen sind, so könnten wir das im Anschluss an Wittgenstein interpretieren, so an bestimmte Modelle ,gewöhnt“, dass sie ihren Modell-Status vergessen und diese zu Entitäten stilisieren, siehe Searle.
} 
darlegen. ProzessontologInnen nehmen es als naturwissenschaftlich gesichert an, dass an der Basis der materiellen Wirklichkeit bestimmte Vorgänge abliefen; mehr noch: dass dynamische Vorgänge, nicht statische Elemente, die Basis der materiellen Wirklichkeit seien. In Anlehnung an die Deutung naturwissenschaftlicher Praxis im Zusammenhang mit Atomtheorien könnte man sich aber fragen, ob wir es bei der dabei anvisierten naturwissenschaftlichen Praxis nicht ebenfalls damit zu tun haben, dass PhysikerInnen, z.B. QuantenphysikerInnen, den Begriff „Prozess“ aus durchaus alltäglichen Kontexten aufgreifen und diesen modellhaft verwenden, um bestimmte empirische Daten im Sinne der Methodik ihrer Wissenschaft zu interpretieren. Das ist vollkommen legitim, insbesondere wenn man der Quantenphysik zugesteht, dass sich für die Modellierung der für sie relevanten Daten statische Gegebenheiten als Ausgangskontexte nicht eignen. Ebensowenig aber wie Thomson behauptet, dass Rosinenkuchen die Mikrobasis der Wirklichkeit sind, behaupten dies wohl QuantenphysikerInnen im Hinblick auf Prozesse. Die kritische Frage ist somit, ob ProzessontologInnen, nicht QuantenphysikerInnen (!), annehmen, methodisch gut eingeführte Modelle wären Entitäten, in diesem Fall dynamische, sprich prozesshafte, um diese dann als ontologische Grundelemente der Wirklichkeit hochzustilisieren. Kommt es nicht auch hier zur Hypostasierung von Modellen und zur ungerechtfertigten Annahme von als Prozessen bezeichneten Vorgängen als Basisentitäten?

Zur Erhärtung des Hypostasierungsmodells gegen die Prozessontologie kann angeführt werden, dass auch ihr spezifischer Vierdimensionalismus als Ontologisierung eines naturwissenschaftlichen Modells, nämlich der Zeit bzw. in der Folge einer Raum-Zeit, aufgefasst werden kann. In der Prozessontologie werden die Basisentitäten standardmäßig als vierdimensionale Raum-Zeit-Gebilde interpretiert. Prozesse haben dort ebenso wie drei räumliche auch eine zeitliche Ausdehnung. Zur Annahme einer vierdimensionalen Raum-Zeit aber beruft man sich auf ein physikalisches Modell, basierend auf der Analogisierung von Zeit und Raum. Dieses Modell wurde u.a. von Hermann Minkowski erfolgreich verwendet, zur Veranschaulichung mancher Merkmale von Raum und Zeit in der speziellen Relativitätstheorie. Behaupten ProzessontologInnen, dass ihre Basisentitäten vierdimensional sind, tun 
sie das also in Folge einer gerade paradigmatischen Hypostasierung eben dieses Modells.

Wenn dem so ist, wenn es naturwissenschaftlicher Forschung um modellhafte Interpretationen empirischer Befunde geht, sind Fragen bzgl. dessen, was es gibt, was existiert, keine naturwissenschaftlichen. Empirische Wissenschaften können somit auch keine Lehrmeister in Sachen Existenz sein. Somit ist es schlicht falsch, dies anzunehmen, insbesondere daraus den Charakter der Ontologie als aposteriorischer Disziplin abzuleiten.

\section{Husserl und Lowe als Zeugen}

Welche Zeugen kann man nun für diese Annahme über die Eigenart naturwissenschaftlicher Theorienbildung im Hinblick auf Existenz vorbringen? Ein erster mag, zumindest auf den ersten Blick gesehen, für einen analytischen Philosophen ungewöhnlich sein. Es ist Edmund Husserl, und zwar unter besonderer Berücksichtigung seiner Schrift zur „Krise der europäischen Wissenschaften““ ${ }^{187}$ Allerdings: Der Punkt, um den es hier geht, ist Verstehen von naturwissenschaftlicher Forschung. Und der lässt sich in der „KrisisSchrift" unabhängig von Husserls sich der Transzendentalphilosophie zuwendenden Phänomenologie darlegen.

Husserls Impulse für das Verstehen naturwissenschaftlicher Forschung gehen von der Annahme aus, dass es sich bei naturwissenschaftlichen Theorienbildungen um ,Veranschaulichungen von Ideen durch Modellbildung“6188 handelt. Auch im Hinblick auf diese Formulierungen können die eben zitierten Atomtheorien als naheliegende Beispiele angeführt werden. Thomson und Rutherford sind ja besonders kreativ, wenn es darum geht, „Ideen“ zur methodischen Interpretation empirischer Daten durch „Modellbildung“ zu „,veranschaulichen“. Auch die Rede von vierdimensionalen Prozessen in physikalischen Theorien kann auf diese Weise verstanden werden.

Bemerkenswert im Hinblick auf die Frage nach Existenz ist, dass Husserl den zitierten Gedanken so einführt, ${ }^{189}$ dass die Rede der Naturwissenschaft-

${ }^{187}$ Hier: Husserl 1954. In der einschlägigen Darstellung Husserls nehme ich Inhalte von Kanzian 2015, insbesondere 71-77, auf. Für eine umfassende Rehabilitierung Husserls, auch des späten, in der Analytischen Philosophie sei auf Meixner 2014 verwiesen.

${ }^{188}$ Vgl. Husserl 1954, 132.

${ }^{189}$ Vgl. ebd., 131. 
lerInnen zwar oft so klingt, als $o b$ die Naturwissenschaften Wissenschaften von einer durch Erfahrung zugänglichen objektiven Natur seien, die dann in Theorien abgebildet würde. Das ist, um bei unseren Beispielen zu bleiben, bei Thomson natürlich weniger anzunehmen als bei Rutherford und bei Prozesstheorien. Unabhängig davon ist aber der entscheidende Punkt klar: Eine physikalische Atomtheorie kann so formuliert sein, als ob es darin um Abbildungen von Gegenständen ginge. Nimmt man das ,als ob“ jedoch für bare Münze, sprich, nimmt man tatsächlich ein Abbildungsverhältnis zwischen Theorie und „Natur an sich“ an, fällt man, so Husserl, einem groben Missverständnis zum Opfer. Denn, so Husserl im Originalton: „Das Objektive ist eben als es selbst nie erfahrbar, und so wird es [...] von den Naturwissenschaften selbst überall da angesehen, wo sie es im Gegensatz zu ihren verwirrenden Reden sogar als ein metaphysisch Transzendentes interpretieren.“ ${ }^{\text {190 }}$ Naturwissenschaften geht es nicht um ,das Objektive als es selbst“. Es geht, so können wir Husserl hier verstehen, in physikalischen Theorien, von ,verwirrenden Reden“ abgesehen, nicht um die Klärung von Existenzfragen, also nicht um den Aufweis von Entitäten. Vom Standpunkt ihrer methodischen Zugangsweise wäre das tatsächlich, wie Husserl sagt, etwas „metaphysisch Transzendentes“. Und metaphysisch Transzendentes ist nicht das Geschäft der Physik.

Dementsprechend spricht Husserl an anderer Stelle auch davon, dass die „theoretischen Idealisierungen der Physiker" Ergebnisse von bestimmten „praktischen Interessen“ $\operatorname{sind}^{191}$ - legitimer praktischer Interessen darf hier ergänzt werden, solange dabei nicht vergessen wird, dass diese Ergebnisse, um bei Husserls Terminologie zu bleiben, ,theoretisch-logische Substruktionen“192 eines ,prinzipiell in seinem Selbstsein nicht Erfahrbaren“"193 sind. Naturwissenschaften erarbeiten legitim interessensgeleitete Theorien, deren Nutzen nach pragmatischen Gesichtspunkten zu beurteilen ist. Wir erwarten ja auch einiges von Naturwissenschaften, Prognosen, technische Anwendungen, die letztlich Menschen in ihren alltäglichen Bedürfnissen zu Gute kom-

\footnotetext{
190 Ebd.

${ }^{191}$ Vgl. ebd., 143.

192 Ebd., 130, 143, 177.

${ }^{193}$ Ebd., 130.
} 
men sollen. Was wir allerdings nicht erhoffen dürfen, ist, dass uns die Physik Erklärungen bzgl. dessen bietet, was die Welt ist, wie wir ihre Grundstrukturen verstehen können. Dazu braucht es ein von ihr unabhängiges, ihr unter dieser Rücksicht vorgelagertes Unternehmen. Das aber ist die Ontologie.

Auch diesen Gesichtspunkt können wir, sogar in klarer Eindeutigkeit, bei Husserl verorten. Dort, wo er davon spricht, dass ,[n]aturwissenschaftliches Wissen [...] an dieses Sein [der Natur] thematisch nie herankommt"“. ${ }^{194}$ Wie gesagt: In den Naturwissenschaften geht es nicht um Sein, nicht um Existenz. Das ist schlicht nicht deren Thema. Bei Husserl hat das zunächst erklärungstheoretische Implikationen, denen zufolge, genau genommen, Naturwissenschaften auch nicht(s) erklären. ${ }^{195}$ Naturwissenschaften „,rastern“ empirische Befunde, und zwar auf eine Weise, die wohl - aufgrund ihrer Interessensausrichtung - immer als abänderbar, um nicht zu sagen pragmatisch eingestuft werden kann. Deshalb gilt für Husserl: „Naturwissenschaftliches Wissen von der Natur gibt also von der Natur keine wirklich erklärende [...] Erkenntnis [... $]^{196}$ Dies müssen wir hier nicht weiter verfolgen. Für uns ist es wesentlich festzuhalten: Geht es den Naturwissenschaften, allen voran der (Quanten-) Physik, gerade nicht um Existenz, ist die Bedingung im Vordersatz des naturalistischen Konditionals, der ja die besondere Autorität von Naturwissenschaften bzgl. Existenz besagt, definitiv hinfällig. Dafür sei Edmund Husserl als Zeuge aufgerufen.

${ }^{194}$ Ebd. „Naturwissenschaftliches“ ist im Zitat der Beginn eines Satzes, somit im Original groß geschrieben.

${ }^{195}$ Vgl. ebd., 193.

${ }^{196}$ Ebd. Einige Zeilen weiter ist Husserl im Hinblick auf den Erklärungsstatus noch radikaler: „Man muss endlich einsehen, dass keine noch so exakte objektive Wissenschaft irgendetwas ernstlich erklärt oder je erklären kann." Bemerkenswert ist, dass sich hier Husserl in Übereinstimmung mit Wittgensteins Tractatus befindet, insofern dieser im Anschluss an eine Erläuterung der (Newtonschen) Mechanik (6.341) klar macht, dass s.E. „,der ganzen modernen Weltanschauung [...] die Täuschung zugrunde[liegt], dass die sogenannten Naturgesetze die Erklärungen der Naturerscheinungen seien.“ (6.371) Bei Wittgenstein finden wir somit auch eine Interpretation der Auffassung von der Erklärungskompetenz der Naturwissenschaft: Es ist eine auf Weltanschauung oder Ideologie beruhende Täuschung. Dass Wittgenstein das Faktum, dass die Welt ist oder existiert, als „,mystisch“ bezeichnet (6.44), lässt ihn ebenfalls in die Nähe Husserls bringen. Jedenfalls ist bestenfalls das „Wie“ der Welt Thema naturwissenschaftlicher Beschreibung, nicht deren Existenz. 
Der späte Husserl steht mit dieser These nicht allein da. Auch Jonathan Lowe, einen paradigmatisch analytischen Ontologen, können wir als Zeugen für diese Annahme anführen. In seinen programmatischen Ausführungen zu Beginn seines Buches „The Possibility of Metaphysics“197 setzt sich Lowe kritisch mit diversen Attacken auf die Metaphysik oder Ontologie als ,erster Philosophie“ auseinander. Dazu gehört auch der Szientismus, den wir hier gut und gern mit dem Naturalismus, wie hier eingeführt, identifizieren können. Jedenfalls stammt die den ontologischen Naturalismus charakterisierende These ,[...] it is these [empirical] sciences, if anything, that can tell us about the fundamental structure of reality" aus Lowes Kennzeichnung dessen, was er Szientismus nennt. ${ }^{198}$

Bei seiner Konterattacke gegen Szientismus bzw. Naturalismus setzt Lowe bei einem Dogmatismus-Vorwurf an. Der Naturalismus macht eine Vorannahme, ohne bereit zu sein, über diese Rechenschaft zu geben. Diese Vorannahme betrifft aber genau das hier zur Diskussion stehende Verhältnis zwischen Naturwissenschaften und Philosophie bzw. Ontologie. Sie besteht darin, dass uns die Naturwissenschaften über die Grundstrukturen der Wirklichkeit Aufschluss geben. Naturwissenschaften erklärten, was es gibt, was existiert. Wir können festhalten, dass dies die Bedingung jenes naturalistischen Konditionals betrifft, dessen Erfüllung die Ontologie zur aposteriorischen Disziplin degradieren würde. Ebenso entspricht Lowes DogmatismusVorwurf dem Hinweis, dass NaturalistInnen aus dem Konditional die bekannte assertorische Behauptung machen: Die Ontologie ist aposteriori.

Lowe bleibt freilich nicht bei der Feststellung stehen, dass der Naturalismus eine nicht-legitimierte zentrale Prämisse voraussetzt. Lowe behauptet auch, dass diese Prämisse falsch ist. Es ist nicht der Fall, dass uns die Naturwissenschaften über Sein und Existenz belehren. Das Umgekehrte gilt. Insofern man naturwissenschaftliche Aussagen als Aussagen über Seiendes bzw. Existierendes interpretieren möchte, muss man das in Anwendung von ontologischen Theorien tun. So gesehen ist die Ontologie die Lehrmeisterin der Naturwissenschaften im Hinblick auf Existenz. ${ }^{199}$

\footnotetext{
${ }^{197}$ Hier: Lowe 1998.

198 Ebd., 4.

${ }^{199}$ Vgl. ebd., 5.
} 
Diese These kann man nun mit Lowe so nachvollziehbar machen, dass er es als Charakteristikum der Metaphysik bzw. Ontologie ${ }^{200}$ ansieht, von Möglichkeiten zu handeln; im englischen Original: „Metaphysics deals in possibilities“. ${ }^{201}$ Für Lowe entscheidend ist, dass wir keine Aussagen darüber, was nun aktuell ist, was existiert, machen können, bevor wir uns über den Bereich des Möglichen in einer systematischen Untersuchung klar geworden sind.

Das ist natürlich klärungsbedürftig. Eine naheliegende Interpretation mag darin bestehen anzunehmen, dass es Aufgabe der Ontologie ist, zunächst einen, wenn man so will „formalen“ kategorialen Rahmen zu entwickeln. ${ }^{202}$ Dieser Rahmen hat universal zu sein, so weit sogar, dass damit alle möglichen Entitäten erfasst sind. Darüber hinaus muss er natürlich konsistent sein, und prinzipiell anwendbar auf bestimmte Erfahrungen, die wir in unserer alltäglichen, aber auch (natur-)wissenschaftlichen Praxis machen. Da es hier um eine Begründung des Vorrangs der Ontologie vor den Naturwissenschaften im Hinblick auf Existenz geht, können wir uns zunächst auf Letzteres, die naturwissenschaftliche Praxis, konzentrieren. Nehmen wir an, diese besteht darin, empirische Befunde aufzuweisen und diese dann mit Hilfe von Modellen $\mathrm{zu}$ interpretieren. Um naturwissenschaftliche Modelle in Aussagen mit Existenzbehauptungen zu verwenden, z.B. „Atome existieren” oder einfacher „Es gibt Atome“, müssen wir den zuvor entwickelten kategorialen Rahmen auf jene Erfahrungen anwenden, die wir in besagter Weise modellhaft interpretieren. Nehmen wir an, jemand sehe, angesichts endurer-freundlicher Ausgangsüberzeugungen, in seinem kategorialen Rahmen die Möglichkeit von Substanzen vor, die sie bzw. er im Sinne aristotelisch-hylemorphistischer Komposita versteht. Die Aussage „Es gibt Atome“ würde dann besagen, dass der Hyle- oder Materialaspekt von Substanzen, physikalisch, sprich nach Auffindung gewisser Daten gemäß bestimmten Atomtheorien modellhaft interpretiert werden kann. Um Lowes Punkt allgemeiner darzulegen: Um modellhafte Interpretationen mit Seiendem oder Existierendem in Zusammen-

\footnotetext{
${ }^{200}$ Lowe spricht stets von „metaphysics“. Wir können bei ihm von einer Synonymie von „Metaphysik“ und „Ontologie“ ausgehen.

${ }^{201}$ Lowe 1998, 5.

202 ... durchaus im Sinne von Ingardens „formaler Ontologie“, die vorab zur Frage nach dem, was materialiter existiert, den Raum möglicher Strukturen der Wirklichkeit abklärt. Vgl. Ingarden 1964, 58ff.
} 
hang bringen zu können, müssen wir diese in Beziehung setzen zu einem vorher entwickelten formal-ontologischen Rahmen. Diese Entwicklung ist Sache genuiner Kreativität, die keinesfalls rein empirisch oder nach Maßgabe naturwissenschaftlicher Modellbildung erfolgen kann. Diese Kreativität ist Sache der Ontologie. In diesem Sinne können wir auch Lowe verstehen, wenn er davon spricht, dass ,ontological notions are not derivate from experience, but are to be invoked in construing what experience reveals of reality". ${ }^{203}$ Existenzbehauptungen in Zusammenhang mit naturwissenschaftlichen Theorien basieren folglich auf Behauptungen darüber, wie unsere Erfahrungen bzw. deren methodische Modellierungen an einen kategorialen Rahmen angepasst bzw. in einen solchen integriert werden können. ,We must judge as best we can how it [the world] is, by determining how well our experience can be accommodated with this or that alternative metaphysical possibility as regards that structure.",204

Insofern also jemand Ergebnisse naturwissenschaftlicher Forschung in Zusammenhang mit Behauptungen darüber, was es gibt, was existiert, bringen möchte, braucht er dazu die Ontologie. Der Naturalismus liegt falsch, wenn er das Gegenteil behauptet, dass nämlich die Ontologie im Hinblick auf Existenz auf Naturwissenschaften angewiesen ist.

\section{Die Ursprungsevidenzen naturwissenschaftlicher Theorien}

Mit Lowe sind wir mit seiner Begründung des angeführten nicht- bzw. antinaturalistischen Vordersatzes ,Wenn es den Naturwissenschaften, allen voran der (Quanten-)Physik, gerade nicht um Existenz geht, somit die Interpretation von physikalischen Forschungsergebnissen im Hinblick auf Existenz autonome Sache der Ontologie ist ..." bereits einen Schritt dazu übergegangen, auch die Plausibiltät des alltagsontologischen Folgesatz darzulegen, der da lautet „... dann (und nur dann) können die Existenzvoraussetzungen alltäglicher Praxis als Universaltheorie alles Existierenden aufgefasst werden. " Und zwar dort, wo angesprochen wurde, dass beispielsweise Modellierungen aus einer physikalischen Atomtheorie durchaus in Zusammenhang gebracht werden

\footnotetext{
${ }^{203}$ Lowe 1998, 10.

${ }^{204}$ Ebd., 23.
} 
können mit einer endurer-Ontologie substanzontologischer Provenienz, die ja eindeutig alltagsontologische Ausrichtung hat.

Dafür soll nun ein weiterer unverdächtiger Zeuge namhaft gemacht und dann nochmals Husserl erwähnt werden. Der unverdächtige Zeuge ist niemand geringerer als Albert Einstein. So weist er in seiner Schrift „Quantenmechanik und Wirklichkeit““205 ausdrücklich auf die Bedeutung des Alltagsdenkens für physikalische Modellbildungen, gerade in der Quantentheorie hin. Einstein räumt ein: „Ohne die Annahme einer solchen Unabhängigkeit der Existenz [...] der räumlich distanten Dinge voneinander, die zunächst dem Alltagsdenken entstammt, wäre physikalisches Denken in dem uns geläufigen Sinne nicht möglich.“' ${ }^{206}$ Die Idee von in räumlicher Distanz stehenden, aber unabhängig voneinander bestehenden Dingen erachtet Einstein für die Physik, insbesondere für Modellbildungen in der Quantenphysik, also als durchaus maßgeblich. Es ist die Idee von Dingen, für die gilt, dass eine äußere Beeinflussung des einen keinen unmittelbaren Einfluss auf das andere hat. Ohne diese Idee wäre das „Prinzip der Nahewirkung“, wie Einstein folgert auch „die Aufstellung empirisch prüfbarer Gesetze in dem uns geläufigen Sinne“ unmöglich. ${ }^{207}$ Diese Idee muss, so können wir Einstein verstehen, von irgendwoher kommen. Aus dem angeführten Zitat aber geht hervor, dass das Alltagsdenken der Ursprung dieser Idee ist, jenes alltägliche Verstehen der Wirklichkeit, in dem Einstein somit auch den Bezug der Begriffe der Physik zu einer „realen Außenwelt“6208 gegründet sieht.

Dies aber können wir umlegen auf den hier zu machenden Vorschlag einer Priorisierung der Existenzannahmen alltäglicher Praxis vor jenen der Physik, somit einer Alltagsontologie vor einer naturalistischen Ontologie. Und zwar so, dass jene Idee, welche Einstein als maßgeblich für physikalische Theorienbildung erachtet, das ist (kurz gesagt) die Idee von unabhängig voneinander bestehenden Dingen, eben auf Existenzvoraussetzungen der alltäglichen Praxis beruht. Es sind also die Existenzvoraussetzungen alltäglicher Praxis, welche den Ausgang bieten zu einem Verstehen physikalischer

\footnotetext{
${ }^{205}$ Hier: Einstein 1948. Die Einstein-Bezüge sind übernommen aus Esfeld 2002.

${ }^{206}$ Einstein 1948, nach Esfeld 2002, 46f.

${ }^{207}$ Vgl. Einstein 1948, nach Esfeld 2002, 47.

${ }^{208}$ Einstein 1948, nach Esfeld 2002, 46.
} 
Praxis, im Hinblick auf deren Modellbildung, aber auch im Hinblick auf deren Weltbezug. Diese Existenzvoraussetzungen aber werden im Kontext einer endurer-Ding-Ontologie systematisch dargelegt. Wenn man bedenkt, dass endurer-Dinge im Kern alltagsontologischer Theorien stehen, könnte man Einstein auch dahingehend interpretieren, dass Alltagsontologie einen Rahmen bietet für physikalische Theorienbildung. Das spricht für deren universale Kompetenz im Hinblick auf Existenz.

Dieser Gedanken kann nun, wie angekündigt, nochmals durch Husserls Krisis-Schrift erläuternd vertieft werden. Und zwar zunächst durch Verweis auf Husserls Konzept der Lebenswelt und deren Bedeutung für naturwissenschaftliche Praxis, in der Folge auf sein Desiderat einer Ontologie dieser Lebenswelt. Zunächst zum Ersten: Ohne hier den Begriff „Lebenswelt“ umfassend einführen zu können, ${ }^{209}$ halten wir fest, dass Husserl damit einen vorwissenschaftlichen, universalen Rahmen meint, der sich ,im sinnhaften Einstellen in Welt, im Bezug auf Welt konstituiert“ ${ }^{210}$ wie Husserl auch sagt, und damit wohl den Bezug der Lebenswelt auf alltägliche (,vorwissenschaftliche") menschliche Einstellungen meint. Was auch immer NaturwissenschaftlerInnen tun, es ist nach Husserl jedenfalls so zu verstehen, dass sie eine nach den Maßgaben ihrer Methoden angestellte Detailbetrachtung dessen vornehmen, was man in eingeführtem Sinn als „Lebenswelt“ bezeichnen kann. Naturwissenschaftliche Forschung ist berechtigte methodische Fokussierung auf einen Aspekt der Welt, unter Ausklammerung seines Bezugs zum Ganzen, eben der Lebenswelt. Daraus ergibt sich aber auch, dass Naturwissenschaft nicht losgelöst oder unabhängig von der Lebenswelt verstanden werden kann. Der Bezug zum Ganzen der Lebenswelt ist vielmehr notwendig, um Naturwissenschaft in dem, was sie ist, adäquat zu verstehen. Naturwissenschaft gründet im Ganzen der Lebenswelt. Ihre „Ursprungsevidenzen“ hat sie aus dem Blick auf das Ganze. ${ }^{211}$ Primär ist, so lässt sich hier Husserl interpretieren, der Blick aufs Ganze, sekundär eine Detailbetrachtung, welche

\footnotetext{
${ }^{209}$ Dazu sei Bermes 2002 empfohlen.

${ }^{210}$ Husserl 1954, 192.

${ }^{211}$ Vgl. Husserl 1954, 132f, 143; ebd. 150 spricht Husserl von der Lebenswelt auch als vom „Boden der Wissenschaften“.
} 
eine legitime, aber methodisch eingeschränkte partielle Sichtweise auf dieses Ganze darstellt.

Damit kann man übergehen zu dem von Husserl genannten Desiderat einer „Ontologie der Lebenswelt“. Husserl geht dabei davon aus, dass die Lebenswelt (schon vorwissenschaftlich) allgemeine Strukturen hat. Daraus ergibt sich die Aufgabe einer Beschreibung dieser Strukturen, die, als „lebensweltliche Ontologie“, ,Wesenslehre jener Onta“ ist, ${ }^{212}$ welche als Elemente die allgemeinen Strukturen der Lebenswelt aufbauen. Die Lebenswelt aber ist wesentlich ,die raum-zeitliche Welt der Dinge, so wie wir sie in unserem vor- und außerwissenschaftlichen Leben erfahren und über die erfahrenen hinaus als erfahrbar wissen. Wir haben einen Welthorizont als Horizont möglicher Dingerfahrung. Dinge: das sind Steine, Tiere, Pflanzen, auch Menschen und menschliche Gebilde“. ${ }^{213}$ Diesen Welthorizont gilt es in einer lebensweltlichen Ontologie zu beschreiben.

Dinge gehören also zu den ontologischen Grundelementen der Lebenswelt, Dinge, die wir gut und gerne als endurer interpretieren können. Solche Dinge gehören jedenfalls auch zu den Existenzvoraussetzungen alltäglicher Praxis, die wir im Sinne einer Alltagsontologie systematisch zu entfalten suchen. Insofern Husserl die Lebenswelt, auch für naturwissenschaftliche Praxis, als rahmen- und ,ursprungsevidenzen“-gebend erachtet, stützt sein Konzept die alltagsontologische These, dass die systematische Darlegung der Existenzvoraussetzungen alltäglicher Praxis als Universaltheorie alles Existierenden aufgefasst werden kann. Diese alltäglichen Existenzvoraussetzungen sind grundlegend für naturwissenschaftliche Praxis, insofern sie auf Existierendes zu beziehen ist.

Die Bestimmung Husserls von raum-zeitlichen Dingen als „Ursprungsevidenzen“ naturwissenschaftlicher Forschung sowie als etwas, das ,wir [...] in unserem vor- und außerwissenschaftlichen Leben erfahren und über die erfahrenen hinaus als erfahrbar wissen“, gibt auch eine Handreichung gegen mögliche Einwände gegen die vorgebrachte alltagsontologische Argumentation, wie etwa: Sind nicht auch Makrodinge, also endurer, Modellierungen, wie es hier von mikrophysikalischen Objekten behauptet wird? Beziehungs-

${ }^{212}$ Vgl. Husserl 1954, 145.

${ }^{213}$ Husserl 1954, 141. 
weise: Wo sind die Grenzen der alltäglichen Lebenswelt? Gehören auch mikrobiologische oder astronomische Objekte dazu?

Hat Husserl Recht, spricht für den Entitätenstatus der alltäglichen Makrodinge, dass wir bei dessen Negierung auf jedwede Ursprungsevidenzen für Naturwissenschaften verzichten müssten. Modellierungen sind Ergebnisse naturwissenschaftlicher Forschung, nicht deren Ursprungsevidenz. Wäre alles Modellierung, destruierten wir letztlich auch jedwede Ontologie, womöglich sogar Richtung Nihilismus. Ja, astronomische Objekte gehören zur husserlschen Lebenswelt, wenn wir sie ,,in unserem vor- oder außerwissenschaftlichen Leben“" als „erfahrbar wissen“, selbst dann, wenn wir sie, z.B. mangels geeigneter Teleskope, faktisch nicht beobachten, also erfahren können. So mancher Planet außerhalb unseres Sonnensystems gehört dazu. Das, so könnte man weiterfahren, ist die Ursprungsevidenz, welche die Astronomie benötigt, wenn und insofern sie auch über Erfahrbares hinaus mathematische bzw. astrophysikalische Modellierungen vornimmt und so zur Astrophysik wird. Analoges gilt für den Bereich der Mikrobiologie. Auch deren Objekte, z.B. Viren, wissen wir in unserem alltäglichen Leben „als erfahrbar“. Bei bestimmten Zellbestandteilen ist das aufgrund ihrer Komplexität zwar fraglich. Das aber macht so lange kein grundsätzliches Problem, bis deren Aufweis von einer möglichen mikroskopischen Erweiterung der Alltagswelt weg übergeht zu einer Modellierung, die sich Elementen derselben als Ausgangskontexte bedient. Damit gehen wir wohl auch über von Biologie zu Biochemie bzw. -physik. Wo Erfahrbarkeit endet und Modellierung aufgrund von Erfahrbarem beginnt, sind auch die Grenzen der Lebenswelt.

Es legte sich durchaus nahe, an dieser Stelle weitere Überlegungen anzustellen bzgl. einer Übereinstimmung von Husserls Ontologie der Lebenswelt mit einer Alltagsontologie, z.B. im Hinblick auf die Kriterien alltagsontologischer Theorienbildung Intuitivität und Revidierbarkeit. Diese lassen sich durchaus auch von Husserl her rekonstruieren, insbesondere wenn man den vorwissenschaftlichen Charakter der Lebenswelt berücksichtigt. Ebenso könnte man Husserl und Einstein in einen näheren Zusammenhang bringen, insofern als beide alltägliches bzw. vorwissenschaftliches Denken als notwendige Voraussetzung physikalischer Modellbildung ansehen. Dies soll hier allerdings zugunsten des anvisierten ,roten Fadens“ zurückgestellt werden. 
Dieser besteht in der Erhöhung der faktischen Plausibilität der im alltagsontologischen Folgesatz angenommenen Möglichkeit „... dann ... können die Existenzvoraussetzungen alltäglicher Praxis als Universaltheorie alles Existierenden aufgefasst werden." Gründe für die Geltung der genannten Möglichkeit sind eo ipso Argumente für die Widerlegung des Naturalismus.

\section{Naturalismuskritik im Kontext: Quine, Hirsch, Viel-Schichten-Modelle}

Der hier anvisierte Anti-Naturalismus ist für den gesamten Duktus dieser Arbeit von entscheidender Bedeutung. Immer wieder wurden Thesen vertreten mit dem Hinweis, dass deren Geltung erst im Kontext einer grundsätzlichen Naturalismuskritik erwiesen werden kann. Deshalb tun wir gut daran, die hier angestellten Überlegungen in Zusammenhang zu bringen mit anderen Theoriestücken. Das sollen paradigmatisch Quines Auffassung von posits und Hirschs Ausprägung der Quantorenvarianz sein. Beschließen wollen wir diesen Abschnitt mit dem Hinweis auf einen weiteren theoretischen Vorteil des vertretenen Anti-Naturalismus in der Ontologie, der sich in der Darlegung eines alltagsontologischen kategorialen Schemas auswirken wird. Es ist die Ablehnung eines „Viel-Schichten-Modells“ der Wirklichkeit zu Gunsten einer lebensweltlichen ,Ein-Schichten“-Auffassung.

Zunächst aber zu Quine. Wie bereits mehrfach angesprochen, steht es im Kern seiner Ontologie, dass er die ontological committments durch Aussagen in naturwissenschaftlichen Theorien als Setzungen (engl.: posits) versteht. Setzungen aber sind convenient intermediaries zwischen begrifflichen Schemata und empirischen Daten. Wir können das auch so verstehen, dass diese Setzungen gemäß bestimmten konzeptuellen Rahmenbedingungen vorgenommene, modellhafte Interpretationen empirischer Befunde sind, die es erlauben, den gewonnenen ,rohen“ Erfahrungsdaten eine praktikable, sprich im Hinblick z.B. auf Prognosen brauchbare Form zu geben. Es wäre eine Überlegung wert, diese Auffassung von Setzungen in Zusammenhang zu bringen mit Husserls „Veranschaulichungen von Ideen durch Modellbildung“, also mit jenen ,theoretischen Idealisierungen der Physiker“, welche, ,von praktischen Interessen geleitet“, naturwissenschaftliche Theorienbildung kennzeichnen. Die Setzung von Molekülen kann jedenfalls, wie gesehen, als Beispiel für jene Modellbildung dienen, die Husserl als „,Veranschaulichung von 
Ideen" im naturwissenschaftlichen Umgang mit Erfahrung annimmt. Der Unterschied besteht freilich darin, dass Quine, wenn man so will, auf halbem Wege stehen bleibt, indem er es als das gesamte Geschäft der Ontologie ansieht, diese Setzungen naturwissenschaftlicher Theorien in logisch durchsichtiger Weise darzulegen. Letztlich macht Quine damit Setzungen zu Entitäten, was genau jenem Fehler entspricht, den wir als den eigentlichen Sündenfall des Naturalismus herausstellen können. Seine Einsicht aber, dass es sich bei dem, worum es in den Naturwissenschaften geht, eigentlich um convenient intermediaries zwischen begrifflichen Schemata und empirischen Daten handelt, kann man auch ohne diesen naturalistischen Fehler weiterentwickeln. Und zwar so, dass man Setzungen als Modelle versteht, die im Rahmen autonomer ontologischer Begriffsbildung im Hinblick auf Existenz zu interpretieren sind. Die naturwissenschaftliche Rede von Molekülen, z.B., besagt demzufolge zunächst, dass sich bestimmte empirische Befunde auf eine Weise modellieren lassen, wie es für gewisse Zielsetzungen, etwa der Chemie, vorteilhaft ist. Dass es Moleküle gibt, im Sinne von Existenz, ist aber keine These der Chemie. Eine Aussage über die Existenz von Molekülen setzt voraus, dass sie in den vorab etablierten Rahmen einer Theorie von Grundstrukturen der Wirklichkeit integriert werden kann; z.B., wie Husserl, Einstein und andere das vorschlagen, in den Rahmen einer Dingontologie. Dann könnte man z.B. behaupten, dass sich Dinge, hinsichtlich ihrer Materialität unter naturwissenschaftlicher Rücksicht eben molekülartig, sprich in Anwendung bestimmter chemischer Modelle, interpretieren lassen. Der entscheidende Punkt ist, dass das, was existiert, dann Dinge sind; Dinge, die natürlich auch naturwissenschaftlich untersucht werden können, vorteilhaft, um bei unserem Beispiel zu bleiben, in Anwendung von Molekül-Modellen.

Die inhaltliche Ausfaltung einer für die Naturwissenschaften rahmengebenden Dingontologie muss hier noch als Postulat angekündigt werden. Dass dies als Ergebnis systematischer Reflexion auf Existenzvoraussetzungen alltäglicher Praxis geschehen kann, sollte allerdings bereits klar sein. Wenn das gelingt, kann man, vielleicht paradoxerweise, Quine und seine Auffassung von Setzungen als richtungsweisend ansehen für das alltagsontologische Verstehen von Ergebnissen naturwissenschaftlicher Praxis, somit für die Etablierung des Primats einer Alltagsontologie vor einer naturalistischen Ontologie. 
Auch Hirsch können wir, wie angekündigt, von hier aus nochmals aufgreifen. Zur Erinnerung: Hirsch zieht die Möglichkeit in Betracht, vom Standpunkt einer als grundlegend angesehenen, sich am common sense orientierenden endurer-Sprache, davon abweichende Sprechweisen, etwa eine perdurer-Sprache, anzunehmen. Wir können als EndurantistInnen so sprechen, als $o b$ wir PerdurantistInnen wären, also auch so, dass wir den Aussagen in der perdurer-Sprache positive Wahrheitswerte zuschreiben.

Im Hinblick auf Alltagsontologie können wir das so interpretieren, dass wir von unserer privilegierten Perspektive aus so sprechen können, als ob die Alternativen adäquat wären: Von einer sich an den Existenzvoraussetzungen alläglicher Praxis orientierenden Sprache aus können wir Redeweisen entwickeln, die so eingeführt sind, als $o b$ wir z.B. den Modellierungen, die wir in der Praxis physikalischer Theorienbildungen verwenden, Entitätenstatus zubilligten. Das ist im Anschluss an Hirsch so $\mathrm{zu}$ verstehen, dass wir von einer Sprache mit der Bedeutung des Quantors $\exists$ im Sinne von $\exists_{\mathrm{E}}$ aus auch Sprechweisen entwickeln können mit „Existenz“ im Sinne von $\exists_{P}$, einem perdurer-Quantor, bzw. von $\mathrm{G}_{\mathrm{T}}$, einem Verstehen von Existenz im Sinne eines Tropen-Quantors. Von unserer durch die Bedeutung von $\exists_{\mathrm{E}}$ geprägten Alltagssprache können wir nun durchaus auch die alternativen Sprechweisen mit positiven Wahrheitswerten versehen; wenn wir nicht vergessen, dass es sich dabei um theoretisch legitime mögliche Abweichungen von der privilegierten Quantorenbedeutung handelt, die faktisch, wie auch Eli Hirsch in vergleichbarem Zusammenhang betont, keine Verpflichtung auf Entitäten mit sich bringen.

Der springende Punkt, der sich aus der vorgelegten Naturalismuskritik ergibt, ist der, dass es sich nunmehr bei der Priorisierung einer sich an der Alltagssprache orientierenden endurer-Sprache nicht, wie bei Hirsch, um eine rein pragmatische Entscheidung handelt. Wenn Naturwissenschaften im Hinblick auf Existenz bzw. Existierendes keine Autoritäten sind, kann die physikalische Sprache nicht als ontologisch grundlegend angesehen werden. Das schließt nicht aus, dass wir so reden könnten, als ob wir über quantenmechanische Prozesse, bzw. nur vage identifizierbare Felder, sogar von Atomen und Molekülen, sprechen. Auszuschließen ist, nunmehr mit Gründen, dass es sich bei den Bezugsobjekten dieser Sprechweisen um Entitäten handelt, aus 
denen dann die Existenzvoraussetzungen alltäglicher Praxis rekonstruiert werden könnten. Kurzum: Ist der Naturalismus falsch, ist das ein entscheidendes, nicht pragmatisches Argument für den grundlegenden Status einer sich am common sense orientierenden endurer-Sprache.

Damit können wir zum angekündigten theoretischen Vorteil des hier vertretenen Anti-Naturalismus in der Ontologie kommen, nämlich die Zurückweisung eines Viel-Schichten-Modells der Wirklichkeit.

Naturalistische Ontologien nehmen standardmäßig eine grundlegende Ebene der Wirklichkeit an, welche aus (vermeintlich - wie wir nunmehr annehmen dürfen) durch die Physik aufgefundenen Basisentitäten besteht. Von dieser grundlegenden Ebene aus werden dann, bottom-up, verschiedene Schichten der Welt, bis hinauf zu unserer alltäglichen Lebenswelt rekonstruiert. Für gewöhnlich beruft man sich auf einschlägige Beziehungen, welche den Aufbau der Schichten oder Ebenen ontologisch gewährleisten sollen. Supervenienz, Emergenz und Konstitution werden hier als Kandidaten angeführt, ohne dass sich in der Literatur ein Vorschlag zur Lösung des Aufbauproblems (auch nur einigermaßen) durchsetzen konnte. An anderer Stelle habe ich versucht, die Problematik dieser Aufbau-Relationen ausführlicher darzustellen. ${ }^{214}$ Die dort vorgebrachte Supervenienz-, Emergenz-, bzw. Konstitutions-Kritik soll hier nicht nochmals aufgerollt werden. Das, und damit komme ich zum eigentlichen Punkt, ist auch gar nicht nötig! Wenn die Naturwissenschaften keine Autorität in Sachen Existenz haben, müssen wir auch keine durch die Physik aufgefundenen Basisentitäten annehmen, welche etwa im Mikrobereich die fundamentale Ebene der Wirklichkeit bildeten; eine Ebene, aus der dann die „oberen“ Schichten zu rekonstruieren wären. Wir dürfen uns eine bottom-up Ontologie, samt den Problemen von Aufbau-Relationen, insgesamt sparen.

Somit können wir, im Gegenteil, die alltägliche Lebenswelt als die grundlegende und, darauf kommt es hier an, einzige Ebene der Wirklichkeit annehmen. Wenn nämlich die systematische Darlegung der Existenzvoraussetzun-

${ }^{214}$ Siehe dazu Kanzian 2016, Abschnitt I - 2.2.3. Dort finden sich auch konkrete Verweise auf solche Supervenienz-, Emergenz- bzw. Konstitutionstheorien. Für „Konstitution“ im Kontext einer alternativen „Metaphysics of Everyday Life“ sei insbesondere auf Baker 2007 verwiesen. 
gen der alltäglichen Praxis eine Universaltheorie alles Existierenden bietet, negieren wir damit, dass es darüber hinaus, oder besser metaphorisch gesagt, „,darunter“ auch noch Entitäten gibt. Das geht zusammen mit der Deutung von mikrophysikalischen Objekten als Modellierungen zur Interpretation von empirischen Befunden (Husserl) bzw. als Setzungen physikalischer Theorien (Quine). Das ergibt sich ferner aus der Deutung einschlägiger Existenzbehauptungen. In Analogie zu den Beispielen aus der Atomtheorie (im Anschluss an Lowe) können wir z.B. „Es gibt kleinste Materieteilchen“, so verstehen, dass es alltägliche Dinge gibt, deren Material im Sinne des aktuellen Standes einer naturwissenschaftlichen Einzeldisziplin teilchenartig zu modellieren ist, und zwar so, dass die Struktur dieser Modellierung echte Teile ausschließt. Wir müssen diese Redeweisen aber nicht als Annahme einer von der alltäglichen Lebenswelt verschiedenen, ja ihr zugrundeliegenden Ebene der Wirklichkeit verstehen.

Ein-Ebenen-Modelle der Wirklichkeit sind keine alltagsontologische Erfindung. Sie werden von OntologInnen favorisiert, die Viel-Schichten-Modellen insgesamt kritisch gegenüberstehen. Diese begründen ihre Skepsis nicht nur mit der angesprochenen Problematik von Aufbau-Relationen, sondern auch mit der Schwierigkeit des Aufweises einer untersten Ebene. Gibt es eine solche, wie kann man diese verstehen? Hängt sie vom aktuellen Stand der Quantenphysik ab? Dann kann sie schwerlich als ontologisch angesehen werden. Gibt es eine solche nicht? Ist dann die Wirklichkeit in einem nicht nur metaphorischen Sinn bodenlos ${ }^{215}$ Auch dieses Dilemma erübrigt sich mit der Annahme der alltäglichen Lebenswelt als einziger Ebene der Wirklichkeit, worin, um es final festzuhalten, ein nicht unerheblicher Vorteil dieser Theorie zu sehen ist.

Fassen wir zusammen: NaturalistInnen behaupten, dass sich die Ontologie in ihrem Kerngeschäft, das ist die Klärung von Existenzfragen, an Ergebnissen von Naturwissenschaften zu orientieren habe. Die Ontologie sei a posteriori. In vorliegender Naturalismuskritik wurde versucht, diese den ontologischen Naturalismus kennzeichnende assertorische Behauptung auf einen Konditio-

\footnotetext{
${ }^{215}$ Vgl. u.a. Schaffer 2003, 501-506, der unterste Ebenen in Viel-Schichten-Modellen weder als empirisch aufweisbar, noch als begrifflich erforderlich darstellt, was zu besagter „Bodenlosigkeit“ führt; aber auch Heil 2003, siehe Abschnitt 2.323.
} 
nal zurückzuführen, das da lautet: Wenn Naturwissenschaften die eigentlichen Autoritäten in Existenzfragen wären, dann (und nur dann) wäre die Ontologie ein aposteriorisches Projekt. In der Folge ging es darum, die Bedingung im Vordersatz als unerfüllt zu erweisen - mit verheerenden Konsequenzen für den Folgesatz. Eine These bzgl. wissenschaftlicher Praxis, gestützt durch Husserl und Lowe, wurde für diesen Erweis dargelegt. Ist der Vordersatz widerlegt, sind wir frei, uns nach einem Ersatz für den naturalistischen Folgesatz umzusehen: Haben die Naturwissenschaften keine Autorität in Sachen Existenz, können wir eine Alltagsontologie, als systematische Theorie der Existenzvoraussetzungen alltäglicher Praxis, als Universaltheorie alles Existierenden auf den Weg bringen. Plausibilitätsgründe für den im alltagsontologischen Folgesatz formulierten Anspruch finden wir darin, dass auch naturwissenschaftliche Theorienbildungen auf Existenzvoraussetzungen alltäglicher Praxis angewiesen sind, sowohl im Hinblick auf ihre Modellbildungen als auch im Hinblick auf den „Weltbezug“ ihrer Forschungsergebnisse. Hier bieten sich Einstein und wieder Husserl als Bezugsquellen an.

Wie eingangs dieses Abschnittes 3.22 gesagt, tun wir als OntologInnen gut daran, in diesen Themenkreisen mit Konditionalen, also bedingt, zu operieren. Und zwar deshalb, weil wir hier letztendlich auf einen Verweis auf außerontologische Praxis, sprich auf einen Dialog mit den Naturwissenschaften angewiesen sind. In diesem Sinne können wir diese Naturalismuskritik mit dem Vorbehalt schließen, dass sie so lange gelten mag, bis uns seriöse NaturwissenschaftlerInnen, nicht naturalistische Deutungen (!) dessen, was NaturwissenschaftlerInnen tun, eines anderen belehren. Der alltagsontologische Optimismus, dass dies nicht so bald geschehen wird, lässt uns zum dritten Feld der Klärung unserer Positionierung übergehen.

\subsection{Alltagsontologie und der Verdacht der Simplifizierung}

In Entsprechung zu den vorhergenden Abschnitten soll dazu zunächst der Begriff „Simplifizierung“ als Bezeichnung einer ontologiekritischen Position in Erinnerung gerufen werden: Die allgemeinste Charakterisierung besteht darin, unter „Simplifizierung“ einen unangemessenen Reflexionsabbruch zu verstehen, also ein Unterlassen bzw. Ausblenden von Überlegungen, die eigentlich von einer Disziplin, in unserem Fall natürlich von der Ontologie, 
angestellt werden müssten. Die Folge ist eine Art von Dogmatismus, der die Ontologie an einem Fortschreiten hindert, oder dieses in eine verfehlte Richtung abgleiten lässt. In der Folge kann man drei Gesichtspunkte von Simplifizierung unterschieden, die faktisch der Ontologie vorgeworfen wurden: Simplifizierung als Ausblenden naturwissenschaftlicher Forschungsergebnisse (siehe 2.31), Simplifizierung unter dem Vorzeichen eines naiven Umgangs mit Sprache; wobei hier nochmals zwischen dem Umgang mit sprachlichen Strukturen im Allgemeinen und sprechakttheoretisch speziellen Elementen (Referenzschemata, naiv hypostasierende Interpretation von Prädikaten) unterschieden wurde (siehe 2.32). Schließlich wurde der Vorwurf an die Ontologie herangetragen, dass sie ihre Fragestellungen illegitimerweise beschränkt, etwa auf Existenzfragen (siehe 2.33).

Wie können wir nun diese allgemein an die Ontologie herangetragene Problematik vom Standpunkt einer Alltagsontologie angehen? Wie, um die Grundfrage dieses Kapitels aufzugreifen, können wir diese kritischen Einstellungen auf Alltagsontologie beziehen, vielleicht sogar für die Weiterentwicklung derselben fruchtbar machen? Auch beim Thema „Simplifizierung“ legt es sich nahe, in einem Dreischritt vorzugehen, wie er bereits beim Dialog mit dem Deflationierungs- bzw. mit dem Naturalisierungsprogramm praktiziert wurde. Wo, erster Schritt, gibt es, auf den ersten Blick gesehen, markante Überschneidungen zwischen dem Anliegen der allgemeinen Simplifizierungskritik an der Ontologie und dem Vorschlag, Ontologie als Alltagsontologie in eingeführtem Sinn zu verstehen? Wo ist gerade Alltagsontologie offensichtlich nicht simplifizierend, könnte man sich auch fragen. Wo aber, zweiter Schritt, empfiehlt es sich, sich von diversen VertreterInnen eines Simplifizierungsvorwurfes zu distanzieren bzw. wo muss man vom Standpunkt einer Alltagsontologie klar gegen diese Vorwürfe Stellung beziehen? Schließlich, dritter Schritt, können wir uns durchaus fragen, wo man einzelne Punkte der Ontologiekritik aufgreifen kann, um sie zur systematischen Fortentwicklung einer Alltagsontologie fruchtbar $\mathrm{zu}$ machen. Hier sollen auch Gesichtspunkte angesprochen werden, die sich als unverzichtbar erweisen für die Verteidigung und Etablierung einer Alltagsontologie als Universaltheorie, sprich als systematische Theorie der Klärung von Existenzvoraussetzungen sämtlicher menschlicher Praxis. Damit wird auch überzuleiten sein 
von den metaontologischen Grundlagen hin zur Entwicklung einer kategorialen Alltagsontologie.

Beginnen wir aber beim ersten Punkt: Welche gemeinsamen Anliegen von Ontologiekritik unter dem Vorzeichen von Simplifizierung und Alltagsontologie fallen bereits auf den ersten Blick ins Auge?

Als erster Gesichtspunkt soll hier Matti Keinänens Kritik am sogenannten Modellismus aufgegriffen werden. Im Kern ist dem Modellismus vorzuwerfen, dass er Modelle zur semantischen Bewertung von bestimmten Aussagen gleichsam hypostasiert und somit - aufgrund der Unterlassung eines eigentlich angebrachten Reflexionsschrittes - aus sprachlichen Schemata ontologische ableitet. Lewis' Possibilismus wurde dabei beispielshaft angeführt. Seine mögliche Welten-Semantik wird zu einer Modalontologie, nach der die aktuelle Welt gleich wie die vielen möglichen Welten existiert. Alltagsontologie ist nun gerade paradigmatisch anti-modellistisch. Das haben wir im Zusammenhang mit der alltagsontologischen Naturalismuskritik gesehen, wo die Hypostasierung von einzelwissenschaftlich legitimen Modellen zur Interpretation empirischer Befunde als Angelpunkt der Zurückweisung naturalistischer Ontologie angeführt wurde. Mit seiner Annahme der Existenz möglicher Welten macht Lewis zwar nicht aus naturwissenschaftlichen Modellen Entitäten, sehr wohl jedoch aus semantischen. Lehnt man das erste ab, so auch das zweite. Es würde hier zu weit führen, einen modalen Aktualismus als alltagsontologische Alternative auszufalten; zumal ja ein nicht-modellierender Possibilismus aus begrifflichen Gründen nicht ausgeschlossen werden kann. Hier wird Lewis' Possibilismus ja nur als Fallbeispiel eines Modellismus unter der Rücksicht Simplifizierung erwähnt. Der steht, um es nochmals auf den Punkt zu bringen, einer Alltagsontologie entgegen.

Ein zweiter Aspekt ist John Heils Simplifizierungskritik unter der Rücksicht einer Zurückweisung naiven Verstehens der Prädikation, näherhin von aussagend gebrauchten Ausdrücken. ${ }^{216}$ Zielscheibe ist jenes Korrespondenzprinzip , $\Phi “$, das Heil so formuliert: „When a predicate applies truly to an object, it does so in virtue of designating a property possessed by that object and

${ }^{216}$ Zum Thema „Prädikation“ siehe: Runggaldier 1990, Kapitel C., Abschnitt V. 
by every object to which the predicate truly applies (or would apply). ‘217 Nach $\Phi$ entspricht, um es vereinfacht zu sagen, jedem (wohlgeformten und wahrheitsgemäß ausgesagten) Prädikat eine Entität aus der Kategorie der Eigenschaften. Damit ist man nicht nur auf Eigenschaften wie Farben, Größen, Massen etc. festgelegt, sondern auch auf abundante Bestimmungen wie rein relationale Eigenschaften, z.B. neben etwas/jemand zu stehen, ja sogar auf formale Charakteristika, z.B. mit sich selbst identisch zu sein, als Entitäten. Auch an dieser Stelle muss zunächst die Einschränkung bekundet werden, dass es hier nicht um die Entfaltung einer alltagsontologischen Theorie von Eigenschaften als Entitäten gehen kann. Wichtig ist allerdings festzuhalten, dass man als Alltagsontologe keinesfalls auf ein $\Phi$-artiges Korrespondenzprinzip festgelegt ist; schon gar nicht, wie es dem Simplifizierungsvorwurf entsprechen würde, ohne kritische Reflexion auf Voraussetzungen eines solchen Prinzips. Im Gegenteil: Man könnte die Kriteriologie für Alltagsontologie sogar zur Stützung einer Anti- $\Phi$-Einstellung heranziehen. So ist es schlicht kontraintuitiv, Prädikate, welche für formale Charakteristika stehen, auf dieselbe Weise zu verstehen, wie Prädikate, durch die z.B. Größeneigenschaften eingeführt werden. Intuitiv merkwürdig ist es festzustellen, dass jemand 1.76 Meter groß und mit sich selbst identisch ist.

Bzgl. der Entsprechung zu Grundstrukturen unseres alltäglichen Sprechens ist zu sagen, dass die Akzeptanz z.B. der Subjekt-Prädikat-Struktur als Richtmaß für kategoriale Schemata keineswegs damit verwechselt werden darf, Prädikate insgesamt, im Sinne von $\Phi$, als „Abbilder“ von Entitäten aufzufassen. Die Subjekt-Prädidat-Struktur als solche ist vielmehr offen für eine differenzierende Interpretation bestimmter prädikativ gebrauchter Ausdrücke im Hinblick auf eine ontologische Bewertung. Will sich Alltagsontologie als systematische Analyse der Existenzvorausssetzungen alläglicher (Sprech)Praxis verstehen, wird sie naive, ja simplifizierende Korrespondenztheorien zurückweisen müssen.

Nicht nur simplifizierenden Deutungen von Prädikaten, sondern auch naiven Referenzschemata stehen alltagsontologische Grundüberzeugungen entgegen. Um das zu zeigen, soll auf den metaontologischen Maximalismus bzw. den Permissivismus (siehe 2.321 bzw. 2.33) Bezug genommen werden.

${ }^{217}$ Heil 2003, 26. 
Nach dem Maximalismus existieren alle quasi-möglichen Objekte (engl.: quasi-possible objects). Quasi-mögliche Objekte aber sind Vorkommnisse einer bestimmten Art F, für die gilt, dass es (irgend-)eine Sprache gibt, in der die Behauptung „Fs existieren“ wahr ist. Entitäten werden zu ,Schatten von singulären Termen“, die in Sätzen solcher Sprachen, nach maximal liberalen Kriterien angenommen, vorkommen. Dem würde auch der Permissivismus beipflichten, demzufolge alles Nichtwidersprüchliche im Wertebereich von Existenzquantoren zu akzeptieren, bzw. für jeden nichtwidersprüchlichen singulären Term eine ihm korrelierende Entität anzunehmen ist.

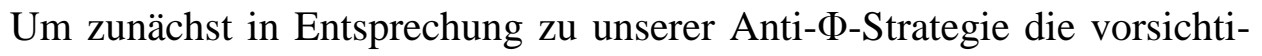
gere Formulierung zu wählen: Keinesfalls ist die Explikation von Existenzvoraussetzungen alltäglicher Praxis auf permissivistische bzw. maximalistische Annahmen festgelegt. Im Gegenteil: „Existenz“ hat nach alltagsontologischen Voraussetzungen eine spezifische Bedeutung, die sich eben am alltäglichen Verstehen orientiert. In diesem Sinn ist, wie gesehen, die endurerSprache grundlegend, das ist jene Sprache, in welcher der endurer-Quantor $\mathrm{B}_{\mathrm{E}}$ als die eigentliche Bedeutung von „Existenz“ angenommen wird. Von dieser eigentlichen Bedeutung können nun per analogiam auch andere ExistenzBedeutungen abgeleitet werden, etwa solche, die der Existenzweise von Ereignissen oder partikularen Eigenschaften entsprechen. Dann aber haben wir auch Bedeutungen von ,Existenz“ ins Auge gefasst, die in einem Als-obSinn eingeführt wurden. Wir können z.B. so sprechen, als ob naturwissenschaftliche Modelle Entitäten wären. In diesem Sinn können wir auch einen perdurer-Quantor $\exists_{P}$ verwenden; sogar in Sätzen, denen wir positive Wahrheitswerte beilegen. Die Einführung aber von Als-ob-Bedeutungen von „Existenz" hat systematisch zu geschehen, das heißt, um auch diesen Gesichtspunkt zu wiederholen, nach Maßgabe nachvollziehbarer Gründe. Bei der Verwendung z.B. einer perdurer-Terminologie mag das unter der Rücksicht einer sinnvollen Interpretation naturwissenschaftlicher Sprechweisen geschehen. Das aber steht dem Maximalismus entgegen, insofern dieser keinen Unterschied macht zwischen im Hinblick auf Existenz eigentlichen Redeweisen und den Als-ob-Sprachen. So werden Modelle naturwissenschaftlicher Theorien genauso als quasi-mögliche Objekte erachtet wie Dinge unserer Alltagswelt. Alltagsontologie ist auch ausdrücklich anti-permissivistisch. Wider- 
spruchsfreiheit ist kein Kriterium für die Entsprechung zur eigentlichen Bedeutung von „Existenz“; bzw., anders gesagt, bei der Reflexion auf die Existenzvoraussetzungen alltäglicher Praxis wird allein Widerspruchsfreiheit zur Klärung dieser Voraussetzungen nicht helfen. Insofern man sowohl Maximalismus als auch Permissivismus einen ungerechtfertigten Abbruch der Reflexion auf ihre theoretischen Voraussetzungen vorwerfen kann, teilen wir als AlltagsontologInnen den Simplifizierungsvorwurf an diese Positionen.

Wir können damit zum nächsten Schritt kommen. Dieser besteht in der Untersuchung, unter welcher Rücksicht wir uns als AlltagsontologInnen von VertreterInnen des Simplifizierungsvorwurfes an die Ontologie dezidiert absetzen müssen. Ein erster Gesichtspunkt ist hier unschwer festzumachen. Insofern nämlich eine Stoßrichtung des Simplifizierungsvorwurfes vor dem Hintergrund des Naturalisierungsprogramms erfolgt, Alltagsontologie aber dem Naturalismus entgegensteht, hat sich Alltagsontologie von dieser Stoßrichtung der Ontologiekritik zu distanzieren.

Führen wir uns dazu nochmals vor Augen, worin der Simplifizierungsvorwurf des Naturalismus besteht: Manche ontologischen Positionen, so meinen NaturalistInnen, blendeten bei ihrem Bemühen, Theorien über die Grundstrukturen der Wirklichkeit zu erarbeiten, Ergebnisse naturwissenschaftlicher Disziplinen, z.B. der Quantenphysik, aus. Das sei ein ungerechtfertigter Reflexionsabbruch, da es ja gerade die Quantenphysik wäre, welche über tiefere und grundlegendere Ebenen der Wirklichkeit Bescheid gäbe. Es existiere mehr, als uns z.B. eine sich am common sense orientierende Ontologie darlegen kann. Diese Kritik an einer common sense Ontologie, wir können durchaus sagen, an einer Alltagsontologie, kann auch so formuliert werden, dass diese mit ihren kategorialen Schemata die Entitäten naturwissenschaftlicher Theorien nicht erreichen könne. Die Kritik auf den Punkt gebracht: An der quantenphysikalischen Basis der Wirklichkeit gebe es keine endurer.

Nunmehr können wir in Anwendung der vorgebrachten Kritik am ontologischen Naturalismus aus 3.223 diesen Simplifizierungsvorwurf zurückweisen, durchaus im Sinne einer nicht-resignativen Erwiderung, wie sie im Abschnitt 2.31 in Aussicht gestellt wurde. Diese kann lapidar darin bestehen, dass es in der Quantenphysik gerade nicht um Existenz, nicht um ,die Wirklichkeit" geht, also auch nicht um deren Grundstrukturen. Somit kann einer 
Ontologie, die quantenphysikalische „Entitäten“, bzw. (vermeintlich) „unter“ der alläglichen Lebenswelt liegende Schichten unberücksichtigt lässt, auch kein Ausblenden eines Bereiches der Wirklichkeit vorgeworfen werden. Was es nicht gibt, kann auch kein legitimes Thema der Ontologie sein.

Freilich, Ontologie muss mit ihren kategorialen Schemata die Ergebnisse naturwissenschaftlicher Forschung erfassen; allerdings so, dass sie deren Modellierungen autonom im Hinblick auf Existenz interpretiert, nicht so, dass sie diese Schemata auf Hypostasierungen dieser Modelle anwendet oder gar daran ausrichtet. Die dabei vorausgesetzte Bestimmung des Verhältnisses zwischen Ontologie und Naturwissenschaften darf nicht dogmatisch erfolgen, sondern durch Anführung von (meta-)ontologischen Gründen. Sind diese aber auf den Tisch gelegt, wie hoffentlich geschehen, kann damit auch die naturalistische Kritik, unter dem Vorzeichen eines Simplifizierungsverdachts, entkräftet werden.

Die Opposition gegen einen weiteren Aspekt des Simplifizierungsverdachts gegenüber der Ontologie ergibt sich nicht so einfach aus bereits Gesagtem. Gemeint ist die Kritik an simplifizierenden Referenzschemata, die manche, u.a. William L. Craig, zu einem Neutralismus in der Deutung der Quantifikation bzw. zu einem referenztheoretischen Deflationismus kommen lässt. Alltagsontologie steht auch diesen Ansätzen entgegen. Die Gründe sollen nunmehr dargelegt werden.

Auch hier beginnen wir mit einigen Bemerkungen zur Wiedererinnerung, diesmal an den Abschnitt 2.322. Dort war davon die Rede, dass Craig zur Vermeidung von simplifizierenden (durchaus im hier eingeführten Sinn) Existenzbehauptungen in der Philosophie der Mathematik, Sprichwort „AntiPlatonismus“, dazu kommt, Quantoren insgesamt als Vehikel zum Ausdruck von Existenzannahmen abzulehnen. Prädikatenlogische Formalisierungen mögen dazu beitragen, logische Ableitungen von Sätzen strukturell transparent zu machen. Ontologische Verpflichtungen gehen wir durch ihre Verwendung (grundsätzlich) nicht ein. Bestenfalls könnte man ja „Es gibt...“ im Sinne eines Partikularquantors meinongianisch auf einen Seinsbereich beziehen. Bei einer angenommenen Trennung zwischen Sein und Existenz ergeben sich daraus aber keine Existenzannahmen. Dem entspricht die referenztheoretische These, dass allein durch die referierende Verwendung von singulären Ter- 
mini kein Bezug zu außersprachlich existierenden Objekten zustande kommt. Es liegt nicht in der „Natur der Referenz“ eine Wort-Welt-Beziehung zu sein.

Es ist hier nicht angebracht, die Kontexte und Voraussetzungen von Craigs Auffassung nochmals aufzurollen. Der springende Punkt ist, dass Alltagsontologie den Vorschlägen Craigs nicht nahetreten muss. Und zwar deshalb nicht, weil Alltagsontologie, vom Ansatz her, simplifizierende Referenzschemata bzw. Quantorenverwendungen, gegen die seine Vorschläge gerichtet sind, ausschließt. Um zunächst bei den Quantorenverwendungen zu bleiben: Für eine Alltagsontologie ist es wesentlich, zwischen „Es gibt...“-Aussagen zu unterscheiden, durch die Existenzvoraussetzungen unserer alltäglichen Praxis zum Ausdruck gebracht werden, und solchen, durch die das nicht geschieht. Dazu gibt es eine genuin alltagsontologische Kriteriologie, von der oftmals die Rede war. Die Durchführung einer solchen Unterscheidung ist im Kontext der Darlegung eines alltagsontologischen kategorialen Schemas vorzunehmen. Den Anfang haben wir in diesem Teil über die metaontologischen Grundlagen allerdings bereits gemacht; etwa durch die Argumentation für den grundlegenden Charakter des endurer-Quantors und analoger Verwendungsweisen, bzw. für seine Abgrenzung zu reinen Als-ob-Verwendungen von „Es gibt ...“-Wendungen. Um Craig noch einmal direkt zu adressieren, ist zu sagen, dass es natürlich noch $\mathrm{zu}$ früh ist, ein alltagsontologisches Versprechen Richtung Anti-Platonismus abzugeben. Zusagen kann man mit ihm allerdings, dass abstrakte Entitäten in der Alltagsontologie sicherlich nicht aufgrund einer als simplifizierend zu bezeichnenden Theorie von Quantoren angenommen werden. Ein Platonismus wäre nur nach erfolgter Prüfung zu akzeptieren, inwiefern Abstrakta zu den Existenzvoraussetzungen alltäglicher Praxis zu zählen sind; bzw. zu den Existenzvoraussetzungen spezieller Praktiken, insofern diese ihre Ausgangsevidenzen aus der alltäglichen Praxis beziehen. Damit können wir auf den von Craig ins Spiel gebrachten Neutralismus verzichten. Er ist, um eine Metapher aufzugreifen, eine Medizin, die Krankheiten heilt, gegen die eine Alltagsontologie immun ist. Aufgrund ihrer schädlichen Nebenwirkungen (siehe wiederum 2.322) halten wir uns lieber von ihr fern.

Analoges gilt für deflationistische Referenztheorien. Sie lösen Probleme, um nun auch unmetaphorisch zu reden, die wir als AlltagsontologInnen 
schlicht nicht haben. Was uns zusätzlich vorsichtig sein lässt, ist, dass durch deflationistische Interpretationen der Referenz, wie gesehen, auch keine kategorialen Schemata ausgeschlossen werden können. Der Weltbezug referierend gebrauchter Ausdrücke kommt nach deflationistischen Referenztheorien durch entsprechende Intentionen zustande. Die aber können eine sehr reichhaltige Ontologie implizieren, welche weit über das hinausgeht, was nach unserer alltagsontologischen Kriteriologie zu den Existenzvoraussetzungen alltäglicher Praxis zu zählen ist. Als AlltagsontologInnen haben wir uns davon zu distanzieren, somit auch insgesamt, um es auf den Punkt zu bringen, von Craigs Sanierungsvorschlägen, die er zur Behebung von Folgen der von ihm attestierten Simplifizierung macht. Alltagsontologie ist von Craigs Simplifizierungs-Kritik nicht betroffen.

Damit kommen wir auf den dritten eingangs erwähnten Gesichtspunkt zu sprechen: Wie lässt sich Alltagsontologie unter Einbeziehung von einschlägigen Aspekten des Simplifizierungsvorwurfs an die Ontologie weiterentwickeln?

In diesem Zusammenhang können wir insbesondere an die Ausführungen im Abschnitt 2.33 anknüpfen, wo jene Ontologiekritik dargestellt wurde, welche auf dem Vorwurf einer ungerechtfertigten Einschränkung ontologischer Fragestellungen beruht. Jene ontologischen Ansätze sind in diesem Sinne simplifizierend, welche unsere Disziplin rein auf Existenzfragen beschränken bzw. eine einfache Auflistung von Kategorien zum Ziel haben. Freilich, Existenzfragen und eine Kategorientafel sind legitime Themen auch einer Alltagsontologie. Allerdings, und das sei als Ansatz den nun folgenden Überlegungen vorangestellt: Vom Projekt einer Alltagsontologie her, wie sie etwa im Abschnitt 3.13 umrissen wurde, ist man keinesfalls darauf verpflichtet, sich auf diese beiden Themen zu beschränken. Das Gegenteil ist der Fall: Die spezifisch alltagsontologische Entfaltung der Existenzvoraussetzungen unserer Praxis wurde immer wieder mit dem Attribut „systematisch“ versehen. Das aber meint auch die methodische Entwicklung einer Universaltheorie bzgl. Existierendem, die über das Verstehen von „Existenz“ und die Aufstellung von Kategorienlisten hinausgeht. Alltagsontologische kategoriale Ontologie ist mehr als eine Auflistung von Existierendem. 
Eine Explikation dieses „mehr“ kann an dieser Stelle ausschließlich im Rahmen des kreativen Umgangs mit den genannten Aspekten des Simplifizierungsvorwurfs geschehen. Aber auch damit begeben wir uns über den Bereich rein metaontologischer Themen hinaus. Eine solche Explikation lässt sich nämlich nicht ohne den Ausblick auf Grundsätze zur Ausführung einer kategorialen Ontologie darlegen. Damit nähern wir uns dem Übergang von Metaontologie zu kategorialer Ontologie; einem Übergang, der durch das Aufgreifen von bereits Gesagtem und das Ausblicken auf noch zu Entfaltendes geschehen soll. In diesem Sinne werden nun einige Aspekte einer solchen nicht-simplifizierenden Alltagsontologie dargelegt.

\section{Varianz}

Beginnen können wir bei der im Rahmen des Simplifizierungsvorwurfs vorgebrachten Kritik an ontologischen Theorien, dass diese starr, ja dogmatisch seien und sich der Frage nach der geschichtlich und kultur-bedingten Varianz bzw. Wandelbarkeit ihrer Thesen (von vornherein) verschließen. Unter Ausklammerung der spezifisch naturalistischen Dimension dieser Kritik sei in diesem Zusammenhang an Susan Haacks Einwände gegen die deskriptive Ontologie Peter Strawsons erinnert (siehe: 2.33 bzw. 3.13). Wir können diese Kritik aufgreifen und sie kreativ zur Weiterentwicklung von Alltagsontologie verwenden. Wenn nötig, mag das auch unter der Rücksicht einer partiellen Absetzung der Alltagsontologie von Strawsons deskriptiver Ontologie, der wir ja ansonsten sehr viel an Inspiration verdanken, geschehen. Es ist am naheliegendsten, auch in diesem Kontext das Kriterium der Revidier- und Entwickelbarkeit für alltagsontologische Thesen aufzugreifen.

Wie, unter Verweis auf die Einführung des Kriteriums in Abschnitt 3.12, unter 3.13 ausgeführt wurde, ist alltägliche Praxis keine monotone und keine statische Größe. Sie kann sowohl synchron in unterschiedlichen Kulturen verschiedene Ausprägungen erfahren als auch diachron in historischer Perspektive. Damit hat man die Varianz von Existenzvoraussetzungen alltäglicher Praxis in einer systematischen Reflexion zu berücksichtigen. Diese Varianz betrifft, wie ebenfalls bereits erwähnt wurde, nicht nur Nebensächliches, sondern, zumindest theoretisch, auch den Kernbereich besagter Voraussetzungen bzgl. Existierendem. Das darf nicht auf die sprichwörtliche leichte 
Schulter genommen werden. So könnte im Prinzip auch das Verstehen von „Existenz“ im Sinne etwa unseres endurer-Quantors davon betroffen sein. Dementsprechend sind Kulturen denkbar, sei es in der Gegenwart, sei es in der Vergangenheit oder der Zukunft, bei denen die Aufgliederung in grundlegende, analoge, und (unter Umständen auch manche) Als-ob-Quantorenverwendungen von der in unserem Alltag gebräuchlichen abweicht. Aus der Sicht unserer faktisch etablierten alltäglichen Praxis, deren Voraussetzungen bzgl. Existenz zentral durch die Annahme von endurer-Dingen, die als Träger von Eigenschaften und deren Änderungen fungieren, anzugeben sind, mag dies ein vernachlässigbarer Legalismus sein. Allerdings kann der Verweis darauf als ein erstes markantes Indiz dafür gelten, dass gemäß der gegebenen Charakterisierung von Alltagsontologie eine einfache Darlegung von Kategorienlisten, etwa Dinge, Eigenschaften, Ereignisse beinhaltend, bzw. der entsprechenden Bedeutung von „Existenz“, für ihre Durchführung nicht genügt. Sie hat mit Varianz zu rechnen. Schon deshalb ist Alltagsontologie vom Ansatz her nicht simplifizierend.

\section{Ontologische Abhängigkeit, Gründung}

Zum selben Ergebnis kann man unter Berücksichtigung eines sachlich gänzlich anders gelagerten Themas kommen. Was reine „Kategorientafel-Ontologien" vernachlässigen, und darauf haben VertreterInnen des Simplifizierungsvorwurfs zurecht hingewiesen, ist eine Untersuchung der Verhältnisse von Vorkommnissen unterschiedlicher Kategorien bzw. eine Darlegung jener Prinzipien, aus denen diese Verhältnisse entwickelt werden sollen. Ontologische Abhängigkeit ist in diesem Zusammenhang der Schlüsselbegriff. Wie kann man nicht nur verstehen, dass z.B. Dinge und ihre Eigenschaften existieren, sondern auch, dass sie durch ein spezifisches Geflecht von Abhängigkeitsbeziehungen miteinander verbunden sind? Weiterführend wäre dann schon die Frage, ob die Abhängigkeit, um beim Beispiel zu bleiben, der Eigenschaften von Dingen, und der Dinge von Eigenschaften symmetrisch ist, oder ob es Unterschiede bei den beiden Richtungen dieser Abhängigkeiten gibt. Lassen sich daraus Schlüsse auf den grundlegenden Charakter einer Kategorie von Entitäten ziehen? 
Dass derlei Überlegungen einer Alltagsontologie nicht nur nicht entgegenstehen, sondern sich sogar von ihrem Ansatz her nahelegen, lässt sich anhand eines bereits dargelegten Kriteriums für den Status von Thesen als alltagsontologische zeigen. Gemeint ist das Kriterium der Entsprechung zu Grundstrukturen unseres alltäglichen Sprechens, zu denen wir nicht nur seine Subjekt-Prädikat-Struktur gezählt haben, sondern auch dessen Mechanismen zur Identifikation von Objekten. Dabei haben wir gesehen, dass unsere Alltagssprache nicht nur Mittel zur Identifikation bestimmter Gruppen von Objekten bereithält, etwa von Dingen bzw. von Eigenschaften, sondern auch, dass die Identifikation bestimmter Objekte abhängt von einer Identifikation anderer Objekte. So können wir, wiederum unter freier Anlehnung an Strawson, davon ausgehen, dass die Identifikation z.B. von Eigenschaften, hier natürlich immer als Partikularien verstanden, auf gewisse Weise von jener der Dinge abhängt; ohne dass dies (auf dieselbe Weise) auch umgekehrt der Fall wäre. Das lässt sich schon daraus ersehen, dass für erfolgreiche Identifikation räumliche Lokalisierung erforderlich ist, und man ohne den Verweis auf Dinge Eigenschaften nicht räumlich verorten kann. ${ }^{218}$ Ohne das an dieser Stelle ausfalten zu können (insbesondere daraufhin, ob nicht auch die Identifikation von Dingen in gewisser Weise von jener der Eigenschaften abhängt), sei festgehalten, dass damit ein erster, genuin alltagsontologischer Ansatz gegeben ist, auch die Verhältnisse von unterschiedlichen Existenzvoraussetzungen unserer alltäglichen Praxis, zu denen Dinge und ihre Eigenschaften wohl zu zählen sind, zu rekonstruieren.

Ein weiterer Ansatz, dem wir in der Entfaltung der metaontologischen Grundlagen von Alltagsontologie bereits begegnet sind, ist die Unterschiedlichkeit der Bedeutung von „Existenz“. Bei unserer Negierung eines univoken Existenzbegriffs haben wir, unter Absetzung von rein äquivoken Interpretationen unterschiedlicher Bedeutungen von „Existenz“ (u.a. Ryle), den Versuch unternommen, ein analoges Verstehen zu implementieren. Ausgehend von der Annahme einer grundlegenden Bedeutung von Existenz, die

218 Abhängigkeit in der räumlichen Lokalisierung als Hinweis auf die ontologische Abhängigkeit nicht-dinglicher Partikularien von Dingen habe ich im Hinblick auf Ereignisse in Kanzian 2001, Abschnitt IV - 3, ausführlich dargelegt. Das dort Dargestellte kann auch auf das Verhältnis zwischen Dingen und partikularen Eigenschaften angewendet werden. 
sich aus der Existenzweise von endurer-Dingen ergibt, haben wir davon abhängige Existenzweisen, etwa jene partikularer Eigenschaften, aber auch von Ereignissen, ins Auge gefasst (v.a. in 3.21 im Rahmen der alltagsontologischen Weiterentwicklung der Quantorenvarianz). An dieser Stelle können wir darauf hinweisen, dass diese Abhängigkeiten von Existenzweisen durchaus geeignet sind, auch die ontologische Abhängigkeit von Entitäten, etwa der Eigenschaften von Dingen, zu erfassen. Wir sehen jedenfalls: Mit der Integration von Dingen und Eigenschaften in eine Kategorientafel sind wir mit ihrer alltagsontologischen Erörterung sicher nicht fertig.

Ontologische Abhängigkeiten, um beim Thema $\mathrm{zu}$ bleiben, sind eine komplexe Angelegenheit. Um nicht auf höherer Ebene, d.h. im Wissen um die Bedeutung dieser Beziehungen, in Simplifizierung zu verfallen, haben wir schon hier, in diesem Übergangsabschnitt, auf Differenzierungen zwischen verschiedenen Weisen ontologischer Abhängigkeit zu verweisen. ${ }^{219}$

Um die spezifischen Differenzen im Bereich ontologischer Abhängigkeiten in den Blick zu bekommen, wurden verschiedene Raster vorgeschlagen. Zum Einstieg sei hier auf Peter Simons Bezug genommen, und zwar unter der Rücksicht, dass er zum einen zwischen individuellen und generischen ontologischen Abhängigkeiten unterscheidet, zum anderen zwischen starken und schwachen. Den Unterschied zwischen starken und schwachen Abhängigkeiten macht aus, dass Letztere die Abhängigkeit des Abhängigen von einem seiner (echten) Teile nicht ausschließt. Stark abhängig ist ein $\mathrm{x}$ jedenfalls von einem $\mathrm{y}$, das außerhalb von $\mathrm{x}$ liegt, sodass y nicht als (echter) Teil von $\mathrm{x}$, und umgekehrt $\mathrm{x}$ nicht als (echter) Teil von y aufgefasst werden kann. Nehmen wir z.B. an, dass ein Ding in diesem Sinn außerhalb einer Eigenschaft liegt, so ist, nach Simons, jede Abhängigkeit der Eigenschaft von diesem Ding stark. Die zuerst genannte Gegenüberstellung zwischen individueller und generischer Abhängigkeit zielt wiederum auf die Unterscheidung ab, ob etwas von einem bestimmten Individuum abhängt oder von irgendwelchen Individuen, allerdings einer bestimmten Art. Klar ist z.B., dass ein Ding, wie eine Kugel, nicht von einer bestimmten seiner Eigenschaften, etwa rot zu sein, abhängt. Sie könnte ja grün übermalt werden. Die Kugel ist also nicht individu-

219 In Kanzian 2016, Abschnitt II - 2.3, habe ich das breiter ausgeführt. Dort (v.a. 104f) finden sich auch die Referenzen zu Simons, von denen im folgenden Absatz die Rede ist. 
ell von dieser Röte abhängig. Das heißt freilich nicht, dass Dinge auf eigentümliche Weise nicht doch von (irgendwelchen) Eigenschaften bestimmter Arten abhängen. Die Kugel muss irgendeine Farbe, auch irgendeine Größe und irgendein Gewicht haben. Eine weitere, bei Simons allerdings nicht vorkommende Unterscheidungsebene bei ontologischen Abhängigkeiten kann man gewinnen, wenn man zwischen einer akzidentellen und einer substantiellen ontologischen Abhängigkeit unterscheidet. Letztere liegt vor, wenn ein $\mathrm{x}$ von einem $\mathrm{y}$ abhängt unter einer für $\mathrm{x}$ wesentlichen Rücksicht; Erstere, wenn dies unter einer für x ,nebensächlichen“ Rücksicht geschieht. Auch hier kann das Verhältnis zwischen Dingen und Eigenschaften als Beispiel fungieren. Während die Abhängigkeit eines Dinges z.B. von seinen Farben als nebensächlich oder akzidentell zu erachten ist, ist das Umgekehrte nicht der Fall. Dieses Rot hier hängt wesentlich davon ab, dass es die Farbe dieser Kugel ist. Dass Eigenschaften von ,ihren“ Dingen in diesem Sinn individuell, stark und wesentlich oder substantiell abhängen, hat so manche dazu veranlasst, den grundlegenden Charakter der Dinge gegenüber ihren Eigenschaften anzunehmen.

Das Attribut „grundlegend“ kann auch in diesem Zusammenhang Anlass sein, ausgehend vom Thema „ontologische Abhängigkeit(en)“ über die, ebenfalls im Abschnitt 2.33 angesprochene und hier bereits angerissene Frage nach Gründung nachzudenken, als Grundbegriff oder Prinzip einer inneren Systematik hierarchischer Ordnung von Vorkommnissen unterschiedlicher Kategorien. Inwiefern kann man etwa davon sprechen, dass Eigenschaften auf Dingen gegründete Entitäten sind, wenn wir die besagten Abhängigkeitsverhältnisse annehmen? Eine Antwort wird natürlich in erster Linie darauf angewiesen sein, wie wir die Gründungs- bzw. Abhängigkeitsbegrifflichkeit, auch im Verhältnis zueinander, genau einführen. Schon jetzt sollte allerdings klar sein, dass sich ausgehend von alltagsontologischen Voraussetzungen diese Fragen stellen. Der Vorwurf der Simplifizierung an manche ontologischen Positionen ist eine Herausforderung, diese Fragen in der Ausarbeitung einer kategorialen Alltagsontologie auch anzugehen.

Die Explikation von ontologischen Abhängigkeiten bzw. von Gründung aber ist eine hochspezialisierte, ja technisch formale Angelegenheit. Da könnte man sich fragen, ob das überhaupt mit dem Rückbezug auf alltägliche Praxis 
zusammengeht, wie er einer Alltagsontologie zu eigen ist. Auslöser einer solchen kritischen Nachfrage könnte die Entsprechung zu alltäglichen Intuitionen sein, als ein Kriterium für die Tauglichkeit von Thesen für eine Alltagsontologie. Unsere alltägliche Praxis ist wohl kaum von Intuitionen über die formalen Charakteristika der Gründungsbeziehung geleitet.

Eine solche Anfrage gibt uns Anlass, eine Klarstellung und eine Spezifizierung vorzunehmen, die beide gerade im Hinblick auf den Simplifizierungsverdacht relevant sind.

Zunächst ist festzuhalten, dass sich die gegebene Kriteriologie für alltagsontologische Thesen, wie sie anfangs im Abschnitt 3.12 entwickelt wurde stichwortartig angeführt: Entsprechung zu alltäglichen Intuitionen, sowie zu den Grundstrukturen alltäglichen Sprechens, schließlich Varianz - an die grundlegenden Linien eines ontologischen Rahmens richtet. Eine solche Linie wäre die Differenzierung zwischen endurer-Dingen, ihren Eigenschaften und ihren Änderungen, als Ereignisse kategorisiert. Diese Differenzierung kann daraufhin untersucht werden, inwiefern durch sie Existenzvoraussetzungen alltäglicher Praxis erfasst werden, wobei die Entsprechung zu den angeführten Kriterien, wie gesehen, eine entscheidende Rolle für ein positives Urteil spielt.

Wenn wir uns aber z.B. der formalen Explikation der Verhältnisse von Vorkommnissen besagter Kategorien widmen, gehen wir von einer Darlegung der Leitlinien zur Klärung der Existenzvoraussetzungen alltäglicher Praxis über zu einer systematischen Entfaltung derselben. Somit wird auch der Bezug zur gegebenen Kriteriologie zu modifizieren sein. Diese Modifikation kann so vorgenommen werden, dass wir auch dann Thesen als alltagsontologische annehmen, wenn sie sich innerhalb eines erwiesenermaßen alltagsontologischen Rahmens entwickeln lassen, selbst hinsichtlich Intuitivität bzw. Entsprechung zu den Grundstrukturen unseres Sprechens neutral bleiben, und, last but not least, mit keinem Anspruch verbunden sind, dem Varianz-, sprich Entwickel- und Revidierbarkeitskriterium, zu widersprechen. ${ }^{220}$ Nehmen wir an, jemand behauptete, dass Eigenschaften, aufgrund ihrer spezifischen ontologischen Abhängigkeit von Dingen, durch Dinge in einem technischen Sinne gegründete Entitäten seien. Dann wäre das im Sinne der

${ }^{220}$ Dies ist eine Weiterentwicklung von Überlegungen aus Kanzian 2009, II - 4. 
vorgeschlagenen Modifikation eine alltagsontologische These, wenn sie im Rahmen einer alltagsontologietauglichen Ding- bzw. Eigenschaftsontologie dargelegt werden kann; wenn sie selbst - wenngleich von alltäglichen Intuitionen nicht erfass- und auf Entsprechung zu sprachlichen Grundstrukturen nicht untersuchbar - auch nicht kontraintuitiv ist bzw. den angenommenen Grundstrukturen der Alltagssprache nicht entgegensteht; sie schließlich auch gegen Weiterentwicklung nicht immunisiert wird.

\section{Wider simplifizerende Ontologisierung alltäglicher Einstellungen}

Einen weiteren Gesichtspunkt des nunmehr anstehenden Themas „Weiterentwicklung von Alltagsontologie unter Berücksichtung von Aspekten des Simplifizierungsvorwurfs" können wir ins Auge fassen, wenn wir bedenken, dass unsere Alltagspraxis im Hinblick auf ihre ontologischen Voraussetzungen stark und chronisch ambivalent ist. Bereits zu Beginn des ersten Kapitels (Was ist Ontologie?) sollte das klar geworden sein, als versucht wurde, die Sensibilität auf eben diese Existenzvoraussetzungen alltäglicher Praxis zu lenken. Existiert alles, wovon z.B. bei der Beschreibung des Beginns eines normalen Arbeitstages die Rede war? Nur zur Erinnerung: nicht nur von Tageszeitung und Wecker, sondern auch von meteorologischen Gegebenheiten, inneren Widerständen, einer möglichen Busfahrt etc. war dort die Rede. Existiert dies alles im Sinne von Grundelementen der Wirklichkeit?

Der nunmehr gemeinte Gesichtspunkt des Simplifizierungsvorwurfs könnte darin bestehen, dass eine Alltagsontologie, der es ausschließlich um „Existenz“ bzw. um die Auflistung von Kategorien ginge, leicht in Gefahr geriete, die paradigmatisch angeführten Redeweisen über die besagten Gegenstände umzulegen auf eine Theorie eben der Existenzvoraussetzungen dieser Praxis. Weil wir im Alltag über dies und jenes reden, existierte es auch, Wecker, genauso wie Befindlichkeit und mögliche Busfahrt. Das wäre allerdings eine sehr naive Umlegung von alltäglichen Einstellungen auf eine Theorie der Grundstrukturen der Wirklichkeit. Sie wäre verheerend für den immer wieder behaupteten systematischen Anspruch einer Alltagsontologie, insbesondere im Hinblick auf eine Universaltheorie alles Existierenden. 
Somit tut man gut daran, alltagsontologische „Sicherungen“ darzulegen, die einer derart naiven Theorienbildung entgegenstehen. Dies soll unter Verweis auf verschiedene Differenzierungsmöglichkeiten erfolgen.

Eine erste Unterscheidung können wir unter Bezugnahme auf bereits behandelte Autoren gewinnen. Es ist die Unterscheidung zwischen ontologisch eigentlichen und ontologisch uneigentlichen Redeweisen. Zunächst sei in diesem Zusammenhang an Theodore Siders ontologese erinnert (siehe 3.223), das er von dem abhebt, was wir hier als ontologisch uneigentliche Redeweisen bezeichnen wollen. (Womit natürlich keineswegs gesagt sein soll, dass in der Alltagsontologie übernommen werden kann, was Sider schlussendlich als ontologisch eigentlich, sprich ontologesisch ausweist. Es geht nur um die Unterscheidung.) Erinnert sei auch an David Chalmers' Hervorhebung von leichtgewichtigen (lightweight) „Es gibt..."-Redeweisen (2.22), von denen er explizit behauptet, dass sie in unserer Alltagssprache durchgängig vorkommen. Denken wir wieder an die schon einmal beispielhaft vorgebrachte dicke Luft, die es (leider) zwischen Hans und Luise gibt. Die hat jedenfalls nichts zu tun mit schwergewichtigen Behauptungen, z.B. über den ontologischen Status von Hans und Luise selbst. Im erwähnten Abschnitt 2.22 wurde auch ein Vergleich von Chalmers' leichtgewichtiger mit Hofwebers interner „Es gibt..."- oder Quantorenverwendung angestellt. Auch Hofweber, davon war schon mehrmals die Rede, unterscheidet zwischen verschiedenen Arten von „Es gibt..."-Formulierungen, was er in seiner Theorie der Unterscheidung zwischen internen und externen Quantoren technisch ausbaut.

Die bei den angeführten Autoren gemachte Unterscheidung können wir nun auch in einer Alltagsontologie machen. Wenn wir unsere alltägliche Praxis im Hinblick auf ihre Existenzvoraussetzungen analysieren, stehen wir zunächst vor der Aufgabe zu untersuchen, welche „Es gibt..."-Wendungen nun eigentlich tatsächlich auf Grundelemente der Wirklichkeit bezogen, welche aber als nicht-ontologesisch, leichtgewichtig, rein intern, sprich ontologisch uneigentlich aufzufassen sind. Ein begriffliches Werkzeug wurde immer wieder angeführt. Es ist die Unterscheidung zwischen einer grundlegenden oder eigentlichen Bedeutung von „Es gibt“ bzw. von „ヨ“, einer (davon) abhängigen, schließlich einer ontologisch uneigentlichen Als-ob-Bedeutung. Ohne dies hier nochmals aufrollen zu können, gar auszuführen, welche Einheiten 
alläglicher Rede in diese Gruppen von Quantorenbedeutungen fallen, soll darauf hingewiesen werden, dass es im Inneren einer Alltagsontologie Theoriestücke gibt, die einer naiven Umlegung von alltäglichen Einstellungen auf Grundstrukturen der Wirklichkeit entgegenstehen. Wir haben jedenfalls die Chance, Unterschiede zwischen Wecker, möglichen Busfahrten und der dicken Luft zwischen Hans und Luise (um bei den gewählten Beispielen zu bleiben) herauszuarbeiten. Der nunmehr gemeinte Aspekt des Simplifizierungsvorwurfs lässt uns jedenfalls, unter der Rücksicht der Differenzierung zwischen ontologisch eigentlichen und uneigentlichen Redeweisen, an die Seite Siders, Chalmers' und Hofwebers rücken.

Eine weitere Ebene der Differenzierung wurde in den bisherigen Ausführungen praktisch noch nicht behandelt. Es ist die Unterscheidung zwischen rein formalen Elementen einer Ontologie und Entitäten auf der einen Seite; bzw. zwischen Entitäten und Epiphänomenen auf der anderen. Die Einschränkung ,praktisch“ soll an jene Passage (in 2.323) erinnern, in der im Anschluss an John Heil und seine Kritik an naiven Abbildtheorien, gemäß

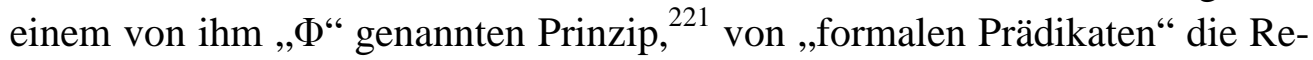
de war. Das sind Prädikate, deren Bedeutung gerade nicht darin besteht, für Entitäten zu stehen. Beispiele sind etwa disjunkte Prädikate wie ,grün oder rot zu sein“, die durchaus auch im alltäglichen Sprachgebrauch vorkommen. Ihre Bedeutung besteht (nach Heil und da schließen wir uns ihm an) nicht darin, für eine disjunkte Entität in der Kategorie der Eigenschaften zu stehen. Das würde eine Unübersichtlichkeit erzeugen, die einer systematischen Erfassung dieser Kategorie entgegensteht. ${ }^{222}$ Vergleichbares gilt für Prädikate, wie z.B. „mit sich selbst identisch zu sein“, wenngleich hier die Begründung des Nicht-Entitätenstatus der Entsprechung des Prädikats anders ausfällt: Mit sich identisch zu sein, ist so grundlegend für jede Entität, dass es selbst nicht als

${ }^{221}$ Zur Erinnerung: ,When a predicate applies truly to an object, it does so in virtue of designating a property possessed by that object and by every object to which the predicate truly applies (or would apply). " Heil 2003, 26.

${ }^{222}$ Diesen Punkt habe ich ausführlich ausgeführt in Kanzian 2016, III - 2.2, im Kontext von Überlegungen zu ,,abundanten Eigenschaften“. Dort gibt es auch grundlegende Überlegungen zum Verhältnis von Prädikaten und Eigenschaften; samt weiterführenden Literaturhinweisen. Für Relationen in diesem Zusammenhang richtungsweisend: Mulligan 1998. 
Entität aufgefasst werden kann. ${ }^{223}$ In beiden Fällen schließt das eine spezifische Weise der ontologischen Interpretation der Prädikate nicht aus. Der springende Punkt ist aber, dass nicht jedem syntaktisch wohlgeformten Prädikat eine Entität entspricht. Akzeptieren wir eine sich an Heil orientierende Anti- $\Phi$-Strategie in unserer Alltagsontologie, wogegen nichts spricht, haben wir ein weiteres Vehikel zur Verhinderung einer naiven Umlegung alltäglicher Redeweisen auf eine Liste von Existierendem.

Analoges gilt von sogenannten Epiphänomenen. Von ihnen war, am Rande, im Kontext des Aufrisses des Simplifizierungsvorwurfs (2.33) die Rede. In der Literatur spielen sie jedoch eine nicht unbeträchtliche Rolle, v.a. dort, wo es darum geht, lebensweltlich oder eben „phänomenal“ irreduzible Elemente zu identifizieren, bei denen es Gründe gegen die Annahme ihres Entitätenstatus gibt. Da sich unsere alltägliche Praxis in lebensweltlichen Kontexten abspielt, wird dies bei einer nicht-simplifizierenden Alltagsontologie eine beträchtliche Rolle spielen. An anderer Stelle habe ich paradigmatisch räumliche oder zeitliche Verhältnisse als solche Epiphänomene dargestellt. ${ }^{224}$ Sie sind unverzichtbar für das Verstehen unserer Praxis in der altäglichen Lebenswelt, ohne dass man Raum und Zeit selbst als Existenzvoraussetzungen dieser Praxis auffassen müsste. Raum und Zeit sind keine Entitäten. Ohne diese Idee an dieser Stelle in bedeutende philosophische Traditionen stellen zu können, wollen wir festhalten: Alltagsontologie ist jedenfalls nicht simplifizierend in dem Sinne, dass sie Redeweisen über räumliche und zeitliche Verhältnisse unreflektiert hypostasiert; selbst wenn, und damit können wir von hier aus an den vorhergehenden Absatz bzgl. ontologisch uneigentlicher Redeweisen anknüpfen, „Es gibt...“-Formulierungen mit ins Spiel kommen, wie z.B.: „Hier gibt es genug Raum“; oder: „Es gibt zu wenig Zeit für dieses oder jenes".

Eine dritte mögliche „Sicherung“ gegen simplifizierende Ontologisierungen alltäglicher Einstellungen sind sogenannte Paraphrasen. Bei ihrer Darlegung können wir wieder bei alltäglichen Redeweisen ansetzen, wie z.B. solchen über natürliche Arten. Im Alltag sprechen wir ja nicht nur über einzelne

\footnotetext{
${ }^{223}$ Vgl. Lowe 2006, 49.

${ }^{224}$ Vgl. Kanzian 2016, Kapitel III - 3, dort wurden epiphänomenale Eigenschaften im Kontext dessen verortet, was man in der Literatur auch ,uneigentliche Eigenschaften“ nennt.
} 
Lebewesen, sondern durchaus auch über Arten oder Spezies derselben; wenn wir z.B. aussagen, dass Spinnen für manche Menschen furchterregende Tiere seien, kurz: „Spinnen sind furchterregend“. Wie können wir derartige, lebensweltlich durchaus relevante Aussagen im Hinblick auf Existenzvoraussetzungen deuten? Sind wir verpflichtet, neben den einzelnen Spinnen auch die Art der Spinnen als Entität anzunehmen, um die Existenzvoraussetzungen der Beispielaussage klar zu machen? OntologInnen mit platonischen Überzeugungen würden diese Frage durchaus bejahen und zusätzlich zu den konkreten, individuellen Spinnen noch abstrakte und universale Arten, wie die der Spinnen, annehmen. Hier kann die Frage nach der Existenz von Abstrakta bzw. von Universalien nicht entschieden werden. Allerdings sei auf ein begriffliches Werkzeug hingewiesen, dessen Anwendung in diesem Fall zur kritischen Prüfung der eigentlichen Existenzvoraussetzungen alltäglicher Praxis dienen mag. Dazu können wir u.a. auf Peter van Inwagen verweisen, der im Anschluss an „Quine's 1946 lecture on nominalism“225 von einer Methode spricht, Aussagen so zu reformulieren, dass der Bezug z.B. auf abstrakte Objekte (wie biologische Spezies) als vermeidbare Redeweise (engl.: „,avoidable manner of speaking“"226) dargelegt wird. In Anwendung auf unser SpinnenBeispiel stellt sich die Frage, ob wir Aussagen, in denen an Subjektstelle Ausdrücke für Arten vorkommen - man also versucht sein könnte, diese als „Es gibt..."-Aussagen über diese Arten zu interpretieren - nicht übersetzen oder reformulieren können in bzw. als Aussagen, in denen an dieser Subjektstelle ausschließlich Termini für individuelle Exemplare, in unserem Fall einzelne Spinnen, vorkommen. Dann wäre der Bezug auf abstrakte Spinnenarten vermeidbar und wären alltagssprachliche Aussagen über sie ontologisch uneigentliche Redeweisen. Derartige Reformulierungen aber sind Paraphrasen. Gelingen die hier exemplarisch erwähnten Paraphrasen, erweist sich, so Quine, die Annahme von abstrakten und universalen Arten insgesamt als unnotwendig, um die Existenzvoraussetzungen, in diesem Fall von arachnophober Praxis, zu klären.

${ }^{225}$ Van Inwagen 2014, chapter 6.

${ }^{226}$ Ebd., 127. 
Wie gesagt, das gute alte Universalienproblem lösen wir hier nicht. ${ }^{227}$ Der springende Punkt für unseren Kontext ist: Beziehen wir die Möglichkeit von Paraphrasen in das alltagsontologische Projekt der Klärung der Existenzvoraussetzungen alltäglicher Praxis ein, haben wir eine weitere „Sicherung“, Simplifizierungen, sprich allzu einfache Umlegungen von alltäglichen Redeweisen auf Existenzannahmen, zu vermeiden. Auch die Prüfung von Paraphrasen einschlägiger Aussagen geht über simplifizierende Existenzanalysen bzw. die Erstellung von Kategorienlisten hinaus. Somit ist auch der Verweis auf sie ein Plädoyer gegen die ungerechtfertigte Einschränkung von ontologischen Fragestellungen, bzw. für eine Systematisierung von ontologischen Annahmen. Wir kommen auch von hier aus zum Ergebnis, dass Alltagsontologie nicht simplifizierend, im Sinne einer standardmäßigen Stoßrichtung der Ontologiekritik ist.

Damit können wir diesen Abschnitt abschließen, in dem es um die Weiterentwicklung einer Alltagsontologie in Auseinandersetzung eben mit dem besagten Simplifizierungsvorwurf geht. Alltagsontologie ist als solche nicht auf Existenzfragen bzw. die Erstellung von Kategorienlisten beschränkt. Sie reflektiert über die mögliche Varianz von Existenzvoraussetzungen alltäglicher Praxis und analysiert die Verhältnisse einzelner Elemente dieser Existenzvoraussetzungen, Stichwort „ontologische Abhängigkeit“ bzw. „Gründung“ von Entitäten. Sie steht simplifizierenden Ontologisierungen alltäglicher Einstellungen entgegen, wie sie reinen „Kategorienlisten-Ontologien“ entsprächen. Mittel dazu ist die Integration von Unterscheidungsmöglichkeiten zwischen ontologisch eigentlichen und uneigentlichen Redeweisen, zwischen formalen bzw. epiphänomenalen Theorieelementen und tatsächlich auf Entitäten bezogenen, schließlich die Prüfung von Paraphrasen verdächtiger „Es gibt ..."Aussagen. Dies alles vermag den systematischen Charakter einer Alltagsontologie - nach den in der Literatur, auch der kritischen, geforderten Standards - zu rechtfertigen. In diesem Sinn können wir übergehen zu einem zusammenfassenden Resümee der hier versuchten metaontologischen Grundlegung einer Alltagsontologie.

${ }^{227}$ Für Ungeduldige: Wer eine solche Lösung versuchen möchte, erprobe sich an Armstrong 1989 bzw. 2010, v.a. chapters 2, 5. 


\subsection{Alltagsontologie: ein Resümee}

Ontologie wurde hier konzipiert als die systematische Reflexion auf Existenzvoraussetzungen menschlicher Praxis; Alltagsontologie aber als jenes Projekt, das mit einer Theorie der Existenzvoraussetzungen alltäglicher Praxis eine Universaltheorie alles Existierenden anvisiert. In der Alltagsontologie ist man dabei geleitet von der Überzeugung, dass sich aus diesen Existenzvoraussetzungen alltäglicher Praxis auch jene spezieller Praktiken, etwa einzelwissenschaftlicher, rekonstruieren lassen.

Um dieses Projekt zu verfolgen, sind wir ausgegangen von Peter Strawsons Annahme des grundlegenden Charakters alltäglicher Denkstrukturen, gegenüber speziellen, wie sie u.a. in Einzelwissenschaften ihre Anwendung finden. Dies entspricht dem Anliegen einer deskriptiven Metaphysik, insofern sie einer revisionären gegenübergestellt ist.

Aus Strawsons Charakterisierung seiner deskriptiven Metaphysik wurden drei Kriterien alltagsontologischer Theorienbildung entwickelt: Intuitivität, Entsprechung zu den Grundstrukturen alltäglichen Sprechens und Entwickelund Revidierbarkeit. Das bedeutet, dass der Status von Thesen als alltagsontologischen daran zu entscheiden ist, ob sie spontanen oder vorwissenschaftlichen Einstellungen von Menschen entsprechen; Kompetenz in der Interpretation der Subjekt-Prädikat-Struktur unserer Sprache bzw. ihrer Identifikationsmechanismen aufweisen; schließlich das Desiderat der kulturellen und historischen Varianz berücksichtigen können. Alltagsontologie ist so gesehen eine Weiterentwicklung von Strawsons deskriptiver Metaphysik. Jedenfalls geben alltagsontologische Thesen Auskunft über Existenzvoraussetzungen alltäglicher Praxis, indem/insofern sie den genannten von Strawson her entwickelten Kriterien entsprechen.

Nota bene: Der angesprochene Praxisbezug, das wurde immer wieder betont, impliziert keinen Pragmatismus, etwa im Sinne von Carnap oder neocarnapianischen Programmen. Die alltagsontologische Untersuchung der Existenzvoraussetzungen von Praxis ist vielmehr (gegen Carnap) kognitiv relevant und nach theoretischen Maßstäben entscheidbar. Auch ist die besagte Entwickel- und Revidierbarkeit alltagsontologischer Thesen nicht im Sinne eines Kultur- oder Geschichtsrelativismus zu interpretieren. Vielmehr ist das Varianz-Kriterium im Kontext der beiden anderen Kriterien zu verstehen und 
steht somit auch in Einklang mit der Annahme eines quer durch Kulturen und Epochen verlaufenden stabilen Kerns von Existenzvoraussetzungen menschlicher Praxis, ohne diese - das ist entscheidend - dogmatisch zu vertreten.

Die Welt existiert jedenfalls ,an sich“. Wie die Welt ist, kann allerdings nur relativ zu unserer Praxis bzw. den Existenzvoraussetzungen derselben verstanden werden. Damit setzt sich dieses Verstehen von ontologischem Realismus ab von dem einer einfachen „Landkarten-Ontologie“, im Sinne etwa des „Writing the Book of the World“-Projekts von Theodore Sider, ohne in einen einfachen Anti-Realismus oder gar Idealismus zu verfallen.

Der Geltungsanspruch von ontologischen Thesen aufgrund der Erfüllung der genannten drei Kriterien ist zunächst auf Alltagsontologie begrenzt. Etwas ist eine (bessere) alltagsontologische These, wenn es den Kriterien entspricht. Damit diese Kriteriologie auch universale Relevanz erhält, muss erst der Vorrang einer Alltagsontologie gegenüber alternativen Entwürfen, etwa revisionären, erwiesen werden. Dann aber wird der Verweis auf die Entsprechung zu den drei Kriterien tatsächlich zu einem ontologischen Argument. Es braucht also eine Begründung, warum Alltagsontologie gegenüber einer revisionären Ontologie der Vorrang zu geben ist; warum - um den hier anvisierten Ontologiebegriff in Anschlag zu bringen - die systematisch dargelegten Existenzvoraussetzungen unserer alltäglichen Praxis tatsächlich rahmengebend sind für das Verstehen der Existenzvoraussetzungen jeder anderen Praxis, u.a. auch einzelwissenschaftlicher Theorienbildung.

Im Kern dieser Argumentation steht Naturalismuskritik, zumal sich revisionäre Ontologie in Existenzfragen auf Ergebnisse einzelwissenschaftlicher Forschung beruft, und genau darin, in der Annahme der Legitimität eines solchen Vorgehens, eine Kernthese des ontologischen Naturalismus besteht: Ontologie ist a posteriori, weil ausgehend von Ergebnissen naturwissenschaftlicher Forschung in Gang zu setzen.

Die vorgebrachte Kritik beruht darauf, den naturalistischen Aposteriorismus auf einen Konditional zurückzuführen: Wenn Naturwissenschaften die Autoritäten in Existenzfragen wären, dann (und nur dann) hätte die Ontologie aposteriorisch vorzugehen. In der Folge ging es darum, die Bedingung im Vordersatz als unerfüllt herauszustellen und den ,naturalistischen Konditional" durch einen alternativen zu ersetzen, im Sinne der Alltagsontologie: 
Wenn es Naturwissenschaften, z.B. der Quantenphysik, gar nicht um Existenz im Sinne der Ontologie geht, sie also bzgl. Existenz keine Autorität beanspruchen können, dann (und nur dann) eröffnet das die Möglichkeit, allägliche Praxis im Hinblick auf Existenzvoraussetzungen zu priorisieren. Plausibilitätsgründe für diese Priorisierung im Hinblick auf einzelwissenschaftliche Praxis rundet die hier vorgebrachte Naturalismuskritik ab.

Bei der Darstellung, warum Naturwissenschaften keine Autoritäten in Existenzfragen sind, wird ausgegangen von einer bestimmten Deutung wissenschaftlicher Praxis. Diese besteht im Kern darin, dass die Entwicklung naturwissenschaftlicher Theorien in der Interpretation von empirischen Befunden durch Anwendung von Modellen geschieht. Die besagten Modellierungen im Kontext naturwissenschaftlicher Theorienbildung lassen von sich aus keine Rückschlüsse auf Existierendes zu. Dies zu missachten und Modelle gleichsam zu hypostasieren, kann als zentraler Fehler des ontologischen Naturalismus angesehen werden. Hier wurde dafür argumentiert, dass die Deutung naturwissenschaftlicher Modellierungen im Hinblick auf Existenz vorab Sache eines von naturwissenschaftlicher Methodik unabhängigen Unterfangens ist, nämlich unserer Ontologie. Dafür wurden prominente Zeugen angeführt: Im Hinblick auf das Verstehen wissenschaftlichen Vorgehens ist das Husserls Krisis-Schrift; unter der Rücksicht der Entwicklung eines kategorialen Rahmens, vor dessen Hintergrund naturwissenschaftliche Ergebnisse bezüglich Existenz erst interpretiert werden können, Jonathan Lowes Possibility of Metaphysics.

Die Quintessenz: Naturwissenschaften sind keine Autoritäten in Existenzfragen. Will die Physik über Existierendes handeln, braucht sie dazu die Ontologie. Wir sind jedenfalls frei zu prüfen, die Existenzvoraussetzungen alltäglicher Praxis zum Ausgang einer Universaltheorie des Existierenden zu machen. Dafür, dass dies faktisch gelingt, ist es u.a. erforderlich zu zeigen, wie diese Existenzvoraussetzungen dazu dienen können, einzelwissenschaftliche Praxis zu verstehen. Das kann gelingen, indem wir u.a. mit Einstein und wieder Husserl annehmen, dass (quanten-)physikalische Modellbildung eben auf „Ursprungsevidenzen aus dem Alltagsdenken“ bzw. „,aus der Lebenswelt“" wie endurer-Dinge angewiesen sind; also gerade auf 
etwas, das im Sinne unserer Alltagsontologie als Existenzvoraussetzungen alltäglicher Praxis interpretiert werden kann.

Der Naturalismus ist aber nur eine Herausforderung für unsere Alltagsontologie. Auch die beiden anderen im zweiten Kapitel geschilderten metaontologischen Positionierungen, das sind Deflationierung und die Ontologiekritik im Sinne eines Simplifizierungsvorwurfs, können dazu beitragen, Alltagsontologie in ihrem theoretischen Profil zu bestimmen. Dabei wurde das Hauptaugenmerk nicht auf Widerlegung von Detailaspekten aus diesen Positionierungen gelegt, sondern auf Integration von Einsichten zur Weiterentwicklung der Alltagsontologie.

Bei der alltagsontologischen Integration von Thesen aus dem Deflationierungsprogramm ist eine Weiterentwicklung von Eli Hirschs Version der Quantorenvarianz von besonderer Relevanz. Es gibt verschiedene Bedeutungen von „Existenz“, welchen unterschiedliche Bedeutungen des Existenzquantors entsprechen. Besonderen Stellenwert nimmt dabei die faktische, als commonsensical zu interpretierende Bedeutung ein. In der Alltagsontologie teilen wir die Annahme, dass die faktische, also alltägliche Bedeutung von „Existenz" ontologische Autorität hat. Dementsprechend nehmen wir auch eine grundlegende Verwendung von „Existenz“ an, die der Existenzweise von endurer-Dingen entspricht. Im Sinne einer Alltagsontologie können wir davon sprechen, dass die Existenzweise dieser endurer-Dinge mit jenen abgeleiteten Existenzweisen von partikularen Eigenschaften und von Ereignissen in engem Zusammenhang steht. Die genannten Existenzweisen sind verschieden, ihnen entsprechende Entitäten können allerdings als unterschiedliche Voraussetzungen derselben Praxis rekonstruiert werden. Das begründet ihren Zusammenhang und bietet einen Anlass, im Kontext der QuantorenvarianzPosition auch ein analoges Verstehen von „Existenz“ bzw. eben des Existenzquantors zu etablieren; unter Absetzung nicht nur von einem äquivoken (Ryle), sondern auch von einem univoken Verstehen (Quine, van Inwagen) von $\mathrm{G}$.

Von beiden, der grundlegenden und der abgeleiteten Bedeutung von „Existenz“, sind Als-ob-Bedeutungen von „Es gibt..."-Wendungen zu unterscheiden. Hirsch selbst führt eine reine perdurer-Sprache als Beispiel einer solchen Als-ob-Redeweise an. Zur Erinnerung: Hirsch meint, wir können so 
sprechen, als $o b$ die Existenzweise von perdurer grundlegend wäre gegenüber jener der endurer. Allerdings zieht sich Hirsch bei der Beurteilung des Status einer „Existenz“-Bedeutung als grundlegend bzw. im Sinne von ,als ob“ auf einen pragmatischen Standpunkt zurück. In der Alltagsontologie wird hingegen versucht, die gesetzten Priorisierungen, vor allem der endurer-Existenzweise, zu begründen - was v.a. im Kontext der Zurückweisung des Naturalisierungsprogramms geschieht.

Schließlich: Auch unter Berücksichtigung von Thesen bzw. Einwänden aus dem breiten Spektrum des Simplifizierungsvorwurf an die Ontologie kann unsere Alltagsontologie weiterentwickelt werden. An dieser zusammenfassenden Stelle soll nur nochmals unterstrichen werden, dass Alltagsontologie - gerade um Universaltheorie der Existenzvoraussetzungen (aller) menschlicher Praxis sein zu können - mehr sein muss als ein einfaches Projekt zur Klärung von Existenzfragen. Das steht simplen Hypostasierungen sprachlicher Strukturen entgegen und gibt Ausblick auf Unterscheidungen auf verschiedenen Ebenen, etwa zwischen ontologisch eigentlichen und, u.a. durch Paraphrasierung aufgewiesenen, uneigentlichen Redeweisen, aber auch zwischen formalen, epiphänomenalen und tatsächlich als Entitäten zu bestimmenden Instanzen zur Klärung der Existenzvoraussetzungen alltäglicher Praxis. Schließlich erfordert die Systematik alltagsontologischer Theorienbildung, die Verhältnisse dieser Existenzvoraussetzungen differenzierend $\mathrm{zu}$ betrachten, Stichwörter „ontologische Abhängigkeit“" bzw. „Gründung“.

Das alles mündet, wie schon mehrmals angedeutet, in ein weiteres Projekt: Was genau sind die Existenzvoraussetzungen alltäglicher Praxis?, folglich: Wie kann man, nach gegebenen Annahmen, daraus einen universalen Rahmen entwickeln, der das Verstehen menschlicher Praxis im Hinblick auf seine Existenzvoraussetzungen ermöglicht?

Mit der Beantwortung dieser Fragen begeben wir uns aus der metaontologischen Grundlegung einer Alltagsontologie hinein in den Bereich einer Entfaltung der Alltagsontologie im Sinne einer kategorialen Ontologie, dem der nächste Teilband dieser Summe einer Ontologie gewidmet sein soll. 


\section{Literatur}

Armstrong 1989: Universals. An Opinionated Introduction. Boulder u.a.: Westview Press.

- 2010: Sketch for a Systematic Metaphysics. Oxford: Oxford University Press.

Baker 2007: The Metaphysics of Everyday Life. Cambridge: Cambridge University Press.

Båve 2009: A deflationary theory of reference. In: Synthese 169 (2009), 51-73.

Bermes 2002: Lebenswelt (abgekürzter Titel). In: Archiv für Begriffsgeschichte 44 (2002), 175-197.

Berto/Plebani 2015: Ontology and Metaontology. A Contemporary Guide. London: Bloomsbury.

Campbell 1990: Abstract Particulars. Oxford: Blackwell.

Carnap 1931: Überwindung der Metaphysik durch logische Analyse der Sprache. In: Erkenntnis 2 (1931), 219-241.

- 1931b: Die physikalische Sprache als Universalsprache der Wissenschaft. In:

Erkenntnis 2 (1931), 432-465.

- 1950: Empirism, semantics, and ontology. In: Revue Internationale de Philosophie 4 (1950), 20-40.

- 1993: Mein Weg in die Philosophie. Stuttgart: Reclam.

Chalmers 2009: Ontological Anti-Realism. In: Chalmers \& other eds. 2009, 77-129. - 2017: The Virtual and the Real; http://consc.net/papers/virtual.pdf (31.8.2017).

Chalmers \& other eds. 2009: Metametaphysics. Oxford: Clarendon Press.

Chisholm 1996: A Realistic Theory of Categories. Cambridge: Cambridge University Press.

Coreth 1999: Ansatz und Vermittlung der Metaphysik. In: Beiträge zur Christlichen Philosophie. Innsbruck: Tyrolia, 207-221.

Correia/Schnieder (eds.) 2012: Metaphysical Grounding. Cambridge: Cambridge University Press. 
Craig 2012: God and Abstract Objects. In: A. Padgett and J. Stump (eds.), The Blackwell Companion to Science and Christianity. Oxford: Wiley-Blackwell, 441-52.

- 2014: Anti-Platonism. In: P. M. Gould (ed.), Beyond the Control of God. New York: Bloomsbury, 113-126.

- 2017: Abstrakte Gegenstände, Naturalismus und Gott. Vortrag gehalten anlässlich des DGPhil Kongresses in Berlin 2017. Unveröffentlichtes Manuskript.

Einstein 1948: Quantenmechanik und Wirklichkeit. In: Dialectica 2 (1948), 320324.

Eklund 2006: Metaontology. In: Philosophy Compass 1/3 (2006), 317-334.

- 2008: The Picture of Reality as an Amorphous Lump. In: T. Sider, J. Hawthorne and D. Zimmerman (eds.), Contemporary Debates in Metaphysics. Oxford: Wiley-Blackwell, 382-396.

Esfeld 2002: Einführung in die Naturphilosophie. Darmstadt: WBG.

Frege 1884: Die Grundlagen der Arithmetik. Breslau: Koebner.

Geach 1968: What Actually Exists I. In: Proceedings of the Aristotelean Society. Suppl. Vol., 42 (1968), 7-20.

Haack 1978: Deskriptive versus revisionäre Metaphysik: Strawson und Whitehead. In: Conceptus 12 (1978), 80-100.

Heil 2003: From an Ontological Point of View. Oxford: Clarendon Press.

Hewitt 2015: A Cardinal Worry for Permissive Metaontology. In: Metaphysica 16 (2015), 159-165.

Hirsch 2002: Quantifier Variance and Realism. In: Philosophical Issues 12 (2002), 51-73.

- 2009: Ontology and Alternative Languages. In: Chalmers \& other eds. 2009, 231259.

Hofweber 2005: A Puzzle about Ontology. In: Nous 39 (2005), 256-283.

- 2009: Ambitious, Yet Modest, Metaphysics. In: Chalmers \& other eds. 2009, 260289. 
- 2016: Carnap's Big Idea. In: S. Blatti and S. Lapointe (eds.), Ontology after

Carnap. Oxford: Oxford University Press, 13-30.

- 2016b: Ontology and the Ambitions of Metaphysics. Oxford: Oxford University Press.

Husserl 1954: Die Krisis der Europäischen Wissenschaften und die Transzendentale Phänomenologie. Hrsg. v. W. Biemel. Husserliana Band VI. Haag: Nijhoff. Ingarden 1964: Der Streit um die Existenz der Welt. Band I: Existentialontologie. Tübingen: Niemeyer.

Kant 1968: Der einzig mögliche Beweisgrund zu einer Demonstration des Daseins

Gottes. Akademie-Textausgabe, Band II, Nachdruck. Berlin: De Gruyter.

- 1968b: Prolegemona zu einer jeden künftigen Metaphysik. Akademie-Textausgabe,

Band IV, Nachdruck. Berlin: De Gruyter.

Kanzian 2001: Ereignisse und andere Partikularien. Paderborn: Schöningh.

- 2009: Ding - Substanz - Person. Frankfurt/M.: Ontos.

- 2015: „Alltagsontologie“ als Lebenswelt-Analyse. In: Phänomenologische

Forschungen (2015). Lebenswelt und Lebensform. Hrsg. v. C. Bermes und A.

Hand. Hamburg: Meiner 2016, 67-82.

- 2016: Wie Dinge sind. Noch eine Alltagsontologie. Berlin/Boston: De Gruyter.

- 2020: Sein als Existenz. In: Handbuch Ontologie. Hrsg. v. J. Urbrich und J.

Zimmer. Berlin: Metzler, 351-357.

Keinänen 2008: Revisionary and Descriptive Metaphysics. In: Philosophica 81 (2008), 23-58.

Koslicki 2012: Varieties of ontological dependence. In: Correia/Schnieder (eds.) 2012, 186-213.

- 2016: Questions of Ontology. In: S. Blatti and S. Lapointe (eds.), Ontology after

Carnap. Oxford: Oxford University Press, 220-241.

Lewis 1986: On the Plurality of Worlds. Oxford: Blackwell.

Liske 2004: Wie soll man Metaphysik betreiben? Deskriptive versus revisionäre

Metaphysik. In: Philosophisches Jahrbuch 111 (2004), 17-42.

Lowe 1998: The Possibility of Metaphysics. Oxford: Clarendon Press.

- 2006: The Four-Categorial Ontology. Oxford: Clarendon Press. 
McDaniel 2009: Ways of Being. In: Chalmers \& other eds. 2009, 290-319.

Manley 2009: Introduction: A Guided Tour of Metametaphysics. In: D. Chalmers \& other eds. 2009, 1-37.

Meixner 2014: Defending Husserl. Berlin/Boston: De Gruyter.

Muck 1999: Rationalität und Weltanschauung. Hrsg. v. W. Löffler. Innsbruck, Wien: Tyrolia.

Mulligan 1998: Relations - Through Thick and Thin. In: Erkenntnis 48, 2/3, (1998). Sondernummer: Analytical Ontology, ed. by C. Kanzian \& E. Runggaldier, 325-353.

Newen/Savigny 1996: Einführung in die Analytische Philosophie. UTB 1878. München: Fink.

Puntel 2006: Struktur und Sein. Tübingen: Mohr Siebeck.

Quine 1948: On What There is. In: Review of Metaphysics 2 (1948), 21-38.

- 1951: Two Dogmas of Empiricism. In: Philosophical Review 60 (1951), 20-43.

- 1960: Word and Object. Cambridge MA: MIT Press.

- 1994: Stimulus and Science. Cambridge MA: MIT Press.

Röd 1989: Existenz als absolute Setzung. In: G. Funke \& M. Seebohm (eds.), Proceedings of the 6th International Kant Congress, Vol. II/1. Washington D.C.: University Press of America, 67-81.

Runggaldier 1990: Analytische Sprachphilosophie. Grundkurs Philosophie, Band 11. Stuttgart u.a.: Kohlhammer.

Runggaldier/Kanzian 1998: Grundprobleme der Analytischen Ontologie. UTB 2059. Paderborn: Schöningh.

Ryle 1949: The Concept of Mind. London: Hutchinson.

Schaffer 2003: Is There a Fundamental Level? In: Nous 37 (2003), 498-517.

- 2009: On What Grounds What. In: Chalmers \& other eds. 2009, 347-383.

Searle 1997: Die Konstruktion der gesellschaftlichen Wirklichkeit. Übers. v. M. Suhr. Reinbek b. Hamburg: Rowohlt. 
Sider 2001: Criteria of personal identity and the limits of conceptual analysis. In:

Philosophical Perspectives 15 (2001), 189-209.

- 2011: Writing the Book of the World. Oxford: Clarendon Press.

Simons 1998: Farewell To Substance: A Differentiated Leave-Taking, in: Ratio (new series) 11 (1998), 235-252.

- 1998b: Metaphysical Systematics: A Lesson from Whitehead. In: Erkenntnis 48, 2/3, (1998), 377-393.

Stoljar/Damnjanovic 2014: The Deflationary Theory of Truth. In: E. Zalta (ed.), The Stanford Encyclopedia of Philosophy (Fall 2014 Edition), https://plato.stanford.edu/archives/fall2014/entries/truth-deflationary (30.6.2020)

Strawson 1972: Einzelding und logisches Subjekt. Stuttgart: Reclam.

Tegtmeier 1997: Zeit und Existenz. Parmenideische Meditationen. Tübingen: Mohr Siebeck.

Thomasson 2015: Ontology Made Easy. New York: Oxford University Press.

Van Inwagen 1990: Material Beings. Ithaca NY: Cornell University Press.

- 1998: Meta-Ontology. In: Erkenntnis 48, 2/3, (1998), 233-250.

- 2014: Existence. Essays in Ontology. New York: Cambridge University Press.

Weingartner 1966 (Hrsg.): Deskription, Analytizität und Existenz. Salzburg: Pustet.

Wittgenstein 1963: Tractatus logico-philosophicus. Frankfurt/M.: Edition Suhrkamp.

- 2005: Vorlesungen über Ästhetik, Psychoanalyse und religiösen Glauben. Frankfurt/M.: Fischer. 


\section{Namensindex}

Anaximander 85

Anaximenes 85

Aristoteles 8, 86f, 90, 153

Armstrong 184

Baker 162

Båve 18, 76, 78

Bermes 156

Berto 4

Campbell 91

Carnap 4-7, 11f, 15, 24-28, 43, 4552, 56, 61, 91ff, 95, 123, 138f, 141. 143,185

Chalmers 13, 52ff, 59, 75, $180 \mathrm{f}$

Chisholm 77

Coreth 2, 11, 126

Correia 86f

Craig 73-78, 93, 170ff

Damnjanovic 17

Einstein 155f, 158, 160, 164, 187

Eklund 71ff

Esfeld 155

Frege 3, 71, 75, 139f

Geach 139

Haack 89, 91, 100, 173

Heidegger 47

Heil 79-82, 93, 163, 166f, 181f

Hewitt 90

Hirsch 32-40, 42, 59, 71f, 94, 128$135,141,159,161,188 \mathrm{f}$

Hofweber 51-57, 59, 75, 92f, 141$144,180 \mathrm{f}$

Hume 125

Husserl 12, 84, 90, 149-152, 155160, 163f, 187

Ingarden 84, 153

Kant 8, 75, 125, 139

Keinänen 67f, 70, 84, 166

Koslicki 86, 91
Lewis 22f, 32, 42, 59, 70, 73, 166

Liske 69

Lowe 11f, 88, 91, 144, 149, 152ff, 163f, 182,187

Manley 19f, 116

Maréchal 11

McDaniel 133f

Meinong 75ff, 86, 140, 170

Meixner 149

Menne 4, 75

Minkowski 148

Muck 2, 14

Mulligan 181

Newen 11

Parmenides 8

Plebani 4

Puntel 108

Putnam 33ff, 72, 128

Quine 3-7, 11f, 15, 22f, 25f, 28, 32, 43, 48-51, 55, 60f, 71, 91, 95, 108, 122, 139ff, 159f, 163, 183, 188

Röd 139

Runggaldier 2, 11, 70, 166

Russell 4, 75

Rutherford 146, $149 \mathrm{f}$

Ryle 33f, 40, 59, 93, 129f, 175, 188

Savigny 11

Schaffer 86-88, 90f, 94, 163

Schnieder $86 f$

Searle 147

Sider 41-43, 57-61, 94f, 125, 144, $180 \mathrm{f}, 186$

Simons 62-65, 68, 83ff, 89ff, 93, 144, 176f

Snape 51,54

Spinoza 88

Stoljar 17 
Strawson 2, 11f, 91, 96-102, 104$109,111,113,173,175,185$

Szatkowski 2

Tegtmeier 3

Thomasson 24-32, 95, 121ff, 126

Thomson 146, 148ff van Inwagen 3f, 22f, 25, 76, 120 , $140,183,188$

Weingartner 4,75

Whitehead 4, 68, 83, 85, 90

Wittgenstein 44-48, 79, 147, 151

Wolff 8,84

\section{Sachindex}

Abstrakta/abstrakt 37f, 53, 74, 76f, $83,171,183$

Analogie/analog 133f, 142, 168, 171, 174f, 188

Äquivokation/äquivok 33, 93, 129f, $132,175,188$

a posteriori/Aposteriorismus 46, 63f, $122,138,143 \mathrm{ff}, 149,152,163 \mathrm{f}$, 186

Atome/Atomismus 22f, 120, 146149, 153f, 161, 163

bottom-up vrs. top-down 64f, 162 Carnap-Quine-Debatte 4, 43 common sense $37,39,61-65,68,93$, 161f, 169, 188 deskriptive Ontologie/ d. Metaphysik

2, 7, 63f, 69, 91, 93, 96-102, 107, 109, 113f, 136, 173, 185

diachrone Identität/d. Selbigkeit 22,

$32,104,127,130,133,140$

easy inferences 27f, 123

Easy Ontology 18, 24-32, 40, 55, 61, 91f, 95, 121-125, 127f, 131

Eigenschaften $21,54,75,79-83,91$, 106, 112, 118, 127, 133, 139f, 167f, 174-179, 181f, 188

Emergenz 162 endurer 22f, 25, 32, 35, 42, 63, 111, $123,127,131,133 \mathrm{ff}, 140,153$, 155ff, 161f, 168f, 174, 176, 178, 187-189

Epiphänomene/epiphänomenal 83, 181f, 184, 189

Ereignisse 83, 105f, 112, 119, 126f, 130, 133, 168, 174-178, 188

Existenz

E.-Kriterien 29f, 124

E.-Quantor: siehe Quantoren

externe E.-Fragen 4f, 24f, 27, 43, $52,54,56,123,126,144$

interne E.-Fragen 4f, 24, 27, 54, 123,126

Sein und E. 76, 139f, 170

formale Eigenschaften/ f. Relationen

$75,80,83,167,181,184,189$

Gründung (grounding) 57, 86ff, 92, 174, 177f, 184, 189

Idealismus/idealistisch, anti-i. 34ff, 98, 125, 128, 186

Identifikation 105f, 108, 112, 119, $133,175,185$

Identitätskriterien $41 \mathrm{f}$ Intuition/Intuitivität, Kontra-I. 102ff, 106-109, 111, 113, 119, 127, 130, 133, 158, 167, 178f, 185 kanonische Notation 49, 108, 140 Kompositionsproblem 22f, 119ff Konstitution 30, 162 
Lebenswelt 1, 31, 64, 156-159, 162f, 170, 182f, 187

linguistic turn 44, 48, 50, 57

Maximalismus 71ff, 78, 93, 167ff

Modelle, Modellbildung 36, 39, 49f, 64, 70, 72f, 134f, 141, 145-149, 153-161, 163f, 166, 168, 170, 187

Modellismus 70, 72, 166

Modest Ontology (bescheidene Ontologie) 51-54, 91ff, 142

mögliche Welten 166

Neutralismus 74-78, 170f

ontologese (Ontologesisch) 59, 144, 180

ontological committments (o.

Verpflichtungen) $6,18,26,49-52$, 55, 74-77, 122f, 135, 140f, 159,

161,170

ontologische Abhängigkeit 84f, 87,

91, 174-178, 184, 189

Paraphrasen 23, 182ff, 189

perdurer 32 , 35, 124, 131, 134f, 161, $168,188 \mathrm{f}$

Permissivismus 86, 88, 90, 94, 167ff Platonismus, Anti-P./platonisch 8, 24, 37f, 53, 73f, 76, 78, 93, 170f, 183

posits (Setzungen) 49f, 60, 122, 141f, 159f, 163

Prädikation/prädizieren 46, 75, 78f, 93, $166 f$

Pragmatismus 27, 40, 50, 185

Prozesse, Prozessontologie 22f, 31, 83, 146-150, 161

Quantoren domain conditions reading (externe Q.-verwendung) 52f, 55f, $75,141 \mathrm{f}, 180$ endurer-Q. 133-136, 140ff, 168, 171,174 generischer Q. 133f

heavyweight (schwergewichtiger Q.) 52ff, 59, 75, 180 inferential role reading (interne Q.-verwendung) 52f, 55f, 59, 75, 141f, 180

lightweight (leichtgewichtiger Q.) 3, 52ff, 59, 75, 78, 180

Nicht-0-Q. 3f, 140

Partikularq. 75,170

perdurer-Q. $135,161,168$ substitutionelle Q.-Verwendung 52

Tropen-Q. 135, 161

Quantorenvarianz 19, 32-35, 37-40, 42, 59, 71, 128-132, 134ff, 142, $159,176,188$

Realismus, Anti-R./realistisch 29, 33f, 41, 50, 53, 58, 60f, 70, 73, 78, $95,122,125 \mathrm{f}, 186$

Referenz 18, 73f, 76ff, 82, 170ff regionale Ontologien 13f, 84 revisionäre Ontologie/ r. Metaphysik 63, 97-101, 106, 108f, 113ff, 117, 120f, 136f, $185 \mathrm{f}$

Semantik, S. und Ontologie 17f, 20, 22ff, 32-36, 38f, 60, 70, 72f, 79, 116f, 119ff, 131, 166

Subjekt-Prädikat-Struktur 97, 104ff, $108,112,119,127,167,175,185$

Supervenienz 30, 87, 162 transzendentale Metaphysik 2, 11, 126

Universalienproblem 54, 118f, $183 \mathrm{f}$ Univozität/univok 60f, 132, 175, 188 Viel-Schichten-Modell 30, 64, 80f, $159,162 \mathrm{f}$ 

Der vorliegende Band ist als erster Teil einer Summe der Ontologie konzipiert. Ziel ist eine Gesamtdarstellung der Ontologie, welche zusammen mit dieser metaontologischen Grundlegung eine darauf aufbauende kategoriale Ontologie und eine angewandte Ontologie umfassen soll. Dieses Buch ist dennoch ein abgeschlossenes Projekt. Sein Anliegen ist die Entwicklung einer Alltagsontologie in kritischkonstruktiver Auseinandersetzung mit Vertreterinnen und Vertretern der aktuellen metaontologischen Debatte. Mit dem Stichwort "Alltagsontologie“ ist auch die Leitidee der intendierten "Summe einer Ontologie" auf den Punkt gebracht: Die Grundstrukturen der kategorialen Wirklichkeit finden wir weder an einer mikrophysikalischen Basis, noch in einem platonischen Ideenhimmel. Ontologie ist eine systematische Beschreibung der menschlichen Lebenswelt. Sie setzt an bei einer Reflexion auf die Voraussetzungen bezüglich Existenz und Existierendem, die wir in unserer alltäglichen Praxis machen. Dieses Programm erfordert fundamentale Naturalismuskritik, damit verbunden eine neue Etablierung der Ontologie als „Erste Philosophie".

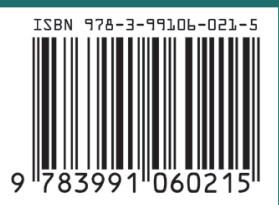

